\title{
The bed bath with or without water? It's a wash!
}

\author{
Citation for published version (APA):
}

Groven, F. M. V. (2021). The bed bath with or without water? It's a wash! Experiences with the washing without water invention used for the bed bath. [Doctoral Thesis, Maastricht University]. Gildeprint Drukkerijen. https://doi.org/10.26481/dis.20211207fg

\section{Document status and date:}

Published: 01/01/2021

DOI:

10.26481/dis.20211207fg

Document Version:

Publisher's PDF, also known as Version of record

\section{Please check the document version of this publication:}

- A submitted manuscript is the version of the article upon submission and before peer-review. There can be important differences between the submitted version and the official published version of record.

People interested in the research are advised to contact the author for the final version of the publication, or visit the DOI to the publisher's website.

- The final author version and the galley proof are versions of the publication after peer review.

- The final published version features the final layout of the paper including the volume, issue and page numbers.

Link to publication

\footnotetext{
General rights rights.

- You may freely distribute the URL identifying the publication in the public portal. please follow below link for the End User Agreement:

www.umlib.nl/taverne-license

Take down policy

If you believe that this document breaches copyright please contact us at:

repository@maastrichtuniversity.nl

providing details and we will investigate your claim.
}

Copyright and moral rights for the publications made accessible in the public portal are retained by the authors and/or other copyright owners and it is a condition of accessing publications that users recognise and abide by the legal requirements associated with these

- Users may download and print one copy of any publication from the public portal for the purpose of private study or research.

- You may not further distribute the material or use it for any profit-making activity or commercial gain

If the publication is distributed under the terms of Article $25 \mathrm{fa}$ of the Dutch Copyright Act, indicated by the "Taverne" license above, 

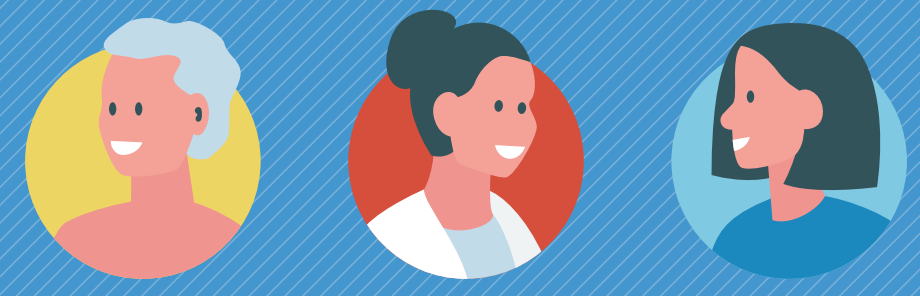

Fabian M.V. Groven

\section{THE BED BATH WITH OR WITHOUT WATER? IT'S A WASH!}

Experiences with the washing without water intervention use d for the bed bath.

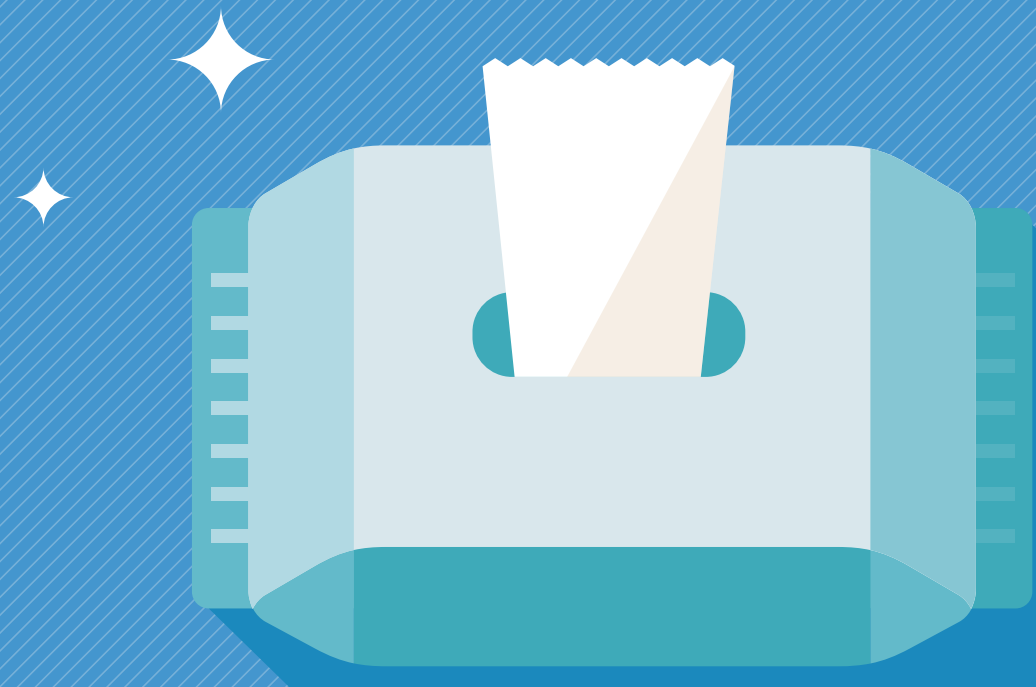



Fabian M.V. Groven

\section{The bed bath with or without water? It's a wash!}

Experiences with the washing without water intervention used for the bed bath.

'It's a wash' Idiom - Used to say that something is equal and that one side does not have an advantage (Merriam-Webster) 
The research presented in this thesis was conducted at CAPHRI Care and Public Health Research Institute, Department of Health Services Research, Maastricht University. CAPHRI participates in the Netherlands School of Public Health and Care Research CaRe.

The research was funded by Arion Holding B.V.

(C) Copyright Fabian Groven, Maastricht 2021

All rights are reserved. No part of this book may be reproduced or transmitted in any form or by any means, without the written permission from the author or, where appropriate, from the publishers of the publications.

Cover and picture design by Boek+Stein

ISBN: 978-94-6419-357-2

Printing by Gildeprint 


\section{The bed bath with or \\ without water? It's a wash!}

Experiences with the washing without water intervention used for the bed bath.

Ter verkrijging van de graad van doctor aan de Universiteit Maastricht, op gezag van de Rector Magnificus, Prof. dr. Rianne M. Letschert, volgens het besluit van het College van Decanen, in het openbaar te verdedigen op dinsdag 7 december 2021 om 13.00 uur

door

Fabian Marcel Victor Groven 


\section{Promotores}

Prof. dr. SMG Zwakhalen

Prof. dr. GJ Odekerken-Schröder

Prof. dr. JPH Hamers

\section{Beoordelingscommissie}

Prof. dr. JMGA Schols (voorzitter)

Dr. BM Olivares Bøgeskov (University College Copenhagen, Denmark)

Prof. dr. JGAM Lemmink

Prof. dr. L Schoonhoven (UMC Utrecht, The Netherlands)

Prof. dr. GDEM van der Weijden 


\section{Contents}

$\begin{array}{lll}\text { Chapter } 1 & \text { General introduction } & 7\end{array}$

Chapter 2 Network well-being from a balanced centricity 25

perspective

Journal of Services Marketing, 2021

Chapter 3 How does washing without water perform

53

compared to the traditional bed bath for

bedridden patients: a systematic review

BMC Geriatrics, 2017

Chapter 4 The effects of washing without water versus

85

the traditional bed bath with water and soap on comfort and physical demands: protocol of a crossover randomized trial

Available online

Chapter 5 Comfort during the bed bath - A randomized

101 crossover trial on the effect of washing without water versus water and soap in nursing students Journal of Clinical Nursing, 2021

Chapter 6 Nurses' beliefs about the bed bath: Testing the self-developed Bed Bath Beliefs tool - A crosssectional study

Submitted for publication

Chapter 7 General discussion

Summary

Samenvatting

Impact

Acknowledgements

About the author

Scientific publications

Living lab in ageing and long-term care 

chapter

1

\section{GENERAL \\ INTRODUCTION}




\section{Mr. Johnson - 87 years old}

Mr. Johnson slowly wakes up in his bed. It takes some time before he realizes again where he is. Not so long ago, he was admitted in a nursing home because his memory problems did not allow him to live at home anymore. At least that is what his daughter and the doctor said. He wants to get up, but a nasty pain prevents him from doing so. He fell earlier this week and fractured his hip. The medical staff expects him to recover, but he will be bedbound for a while. He lies back again and tries to take a comfortable position. He starts to wonder what the

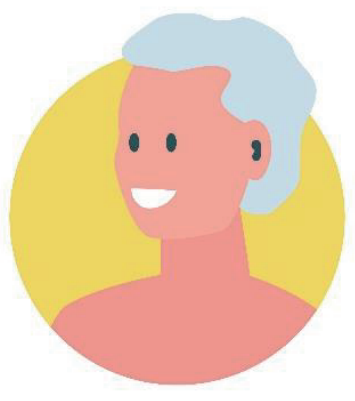

Figure 1 - Mr. Johnson day will bring. Probably, one of the nurses will come in soon for the morning care activities. He hopes he will get a proper bed bath as he is looking forward to his daughter who will visit him later today. After his wife died and he was admitted to the nursing home, his daughter's visits are one of the few things left that really give him joy. He enjoys the time he can be close to her. When he is properly bathed, he also feels he is able to do so. He thinks: "I hope that the nursing staff will not take much longer before they bathe me. I feel hungry and would like to start my day!"

For most of us, bathing (and personal hygiene, in general) not only feels as a daily necessity, but can also be a pleasurable activity. Personal hygiene has many functions, including maintaining good health, preventing unpleasant odors, and feeling comfortable in engaging in social activities with others. That is, many of us take a shower before we go out to meet friends or relatives. Sometimes, people undertake personal hygiene activities purely for the sake of relaxation. For example, many feel reborn after a fresh shower in the morning. However, for Mr. Johnson, who has to rely on others for his personal hygiene, bathing can be anything but relaxing. And he is not the only one.

Many older persons are still able to take care of their personal hygiene themselves, though with some struggles. Some need assistance from others but are still able to take a shower or bath. Approximately $21 \%$ of people older than 85 experience bathing disabilities to such an extent that bathing assistance is required.' Bathing disabilities often mark the start of functional decline and are related to hospital use and nursing home admissions. ${ }^{2}$ Unfortunately, some hospital patients and nursing home residents are bedbound or immobile to such an extent that it is too burdensome to take a shower or bath (every day). These people often receive bed baths from nursing staff.

The bed bath is an important daily activity for bedridden patients, yet can be stressful for patients and nurses. ${ }^{3}$ Predominantly, the decreasing nurse-patient ratio (i.e. the shortage of nurses in combination with an aging population) will put more (time-) pressure on nurses to deliver daily care, including the bed bath. ${ }^{4}$ The increased (time-) pressure could lead to a focus on efficiency and subsequently, a task-oriented approach for the bed bath, at the expense of paying attention to patients' 
experiences. ${ }^{5}$ Such an approach also prevents nurses from building intimate relationships with their patients. Consequently, patients and nurses can have negative experiences with the bed bath. Before we will present this dissertation's topic related to the bed bath, we present the two ways in which the bed bath can generally be executed.

Since the time of Florence Nightingale, the predominant way to bathe patients in bed is the water and soap bed bath. To bed bathe a patient, two washbasins, three cotton towels, two washing mitts or cloth, water, soap, and possibly, body lotion are used. At the end of the $20^{\text {th }}$ century, Susan Skewes, yet another nurse, invented an alternative bed bath method. She had doubts about the water and soap bed bath, especially with respect to the risk of cross-contamination. She concluded that using the same water and washcloth to bathe a whole patient's body was not very hygienic, and therefore thought about possible alternatives. Eventually, she cut a towel in eight pieces and wet them in water. Then, she used one piece for each separate body part. Not much later, the industry transformed this idea in the innovation commonly referred to as washing without water, which is the central topic of this dissertation.

We describe washing without water (sometimes called "disposable bathing") as the use of disposable, prepacked products for a full body wash, consisting of a nonwoven carrier (washcloth) and a no-rinse cleansing fluid that allows nursing staff to bathe someone without the use of water. ${ }^{3}$ Several brands of washing without water products that meet our description are currently used in health care practice. These products all contain a number of wipes or gloves (cloths that covers the complete hand) that contain a skin-cleaning and often skin-treating no-rinse lotion that evaporates by air. One package (often containing eight wipes or gloves) is used for a complete body wash of one patient, and every body part is bathed with one wipe or glove (as depicted in Figure 2).

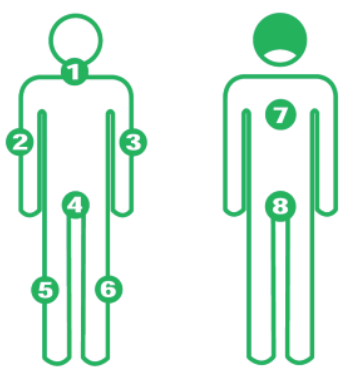

Figure 2 - User instructions for washing without water

Although the washing without water concept has its roots in the early nineties of last century, in 2021, many health care institutions still think of it as an innovation. To define innovation, we refer to Greenhalgh et al., who defined service delivery innovation as "a novel set of behaviors, routines, and ways of working that are discontinuous with previous practice, are directed at improving health outcomes, administrative 
efficiency, cost effectiveness, or users' experience and that are implemented by planned and coordinated actions" (p. 582).6 The washing without water bed bath complies with this definition, as it is a novel way of working (i.e. bed bathing), which is claimed to improve health outcomes, efficiency, and user experiences.

Some advantages have been attributed to the washing without water bed bath. Susan Skewes claimed that the washing without water method cleans and cares for the skin at a high level of convenience, based on nurses' and patients' experiences. ${ }^{7}$ Furthermore, washing without water would counter the problems of the traditional water and soap bed bath, with respect to hygiene and the risk of crosscontamination. The use of washing without water eliminates the use of wash basins and water, which are both sometimes found to contain pathogens related to hospital-acquired infections.8, 9 In addition, towel drying, which could negatively affect the condition of the skin, is no longer required when washing without water is used. 10 Because each washcloth is only used for one body part, it is unlikely that bacteria will be spread from one body part to the other. Finally, washing without water would allow a more standardized, and therefore consistent, technique."

Despite these claims, the traditional bed bath with water and soap is the standard in most health care institutions due to several reasons, including perceived availability and cost-effectiveness of the traditional water and soap bed bath. ${ }^{12}$ Another reason why the traditional bed bath is provided most, is that people grow up with the notion that water is an indispensable requirement for a proper body wash. Because water and soap represent the cultural norm, nurses and patients may hold doubts about the possible advantages of the washing without water innovation. ${ }^{13}$ Such doubts may exist due to the lack of scientific evidence on washing without water that support the claimed advantages of the washing without water bed bath. Furthermore, much is unknown about the experiences with this type of bed bathing. Therefore, scientific evidence regarding experiences with washing without water is warranted,

This dissertation is about the washing without water intervention. As with any intervention, it is important to consider the context in which it might be used, which is the bed bath for the washing without water intervention. Therefore, the bed bath in general will be explored from two perspectives: a service marketing perspective and a health care perspective. From the service marketing point of view, the bed bath can be seen as a health care encounter in which value is co-created by multiple actors, which affects their well-being. From the health care point of view, the bed bath is argued to be an essential of care that is important to patients' and nurses' well-being.

By assessing experiences with washing without water, well-being is considered. Both the service marketing and health care perspective recognize the importance of the interplay between different actors on well-being. Therefore, three actors that are important to the bed bath will be considered in this dissertation: bedridden care recipients (often referred to as patients in this dissertation), nurses, and family members. We have already introduced the patient (Mr. Johnson); it is now 
time to meet the other actors of the network: Nurse Davies and daughter Quinn (the family member). After we meet the actors, the bed bath is described from the service marketing and health care perspectives.

\section{Nurse Davies - $\mathbf{4 5}$ years old}

It is $10 \mathrm{AM}$ and Nurse Davies finds herself at work in the nursing home, in the middle of the morning activities. These activities include waking residents, undressing residents, bathing residents, getting residents dressed, taking care of residents' teeth and hair, getting residents out of bed, giving residents medication, and sometimes, giving residents something to eat and drink in their own room. This morning, she needs to take care of six residents, of which four are bedridden. For those residents, bathing especially takes a lot more time. She

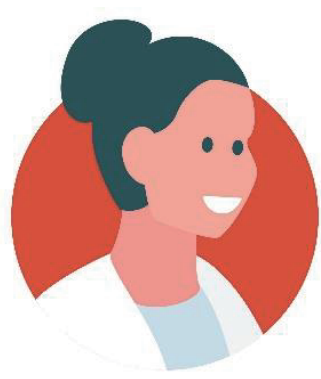

Figure 3 - Nurse Davies still needs to take care of two bedridden residents and she is now heading to one of them, Mr. Johnson. Nurse Davies knows that Mr. Johnson prefers to receive his morning care around 8 AM, but the nursing team decided to take care of him later because, at this time of the day, there is more staff available to lend a hand. Since Mr. Johnson broke his hip, it is more convenient for the nurse, but also for Mr. Johnson, to turn him in bed with the help of another nurse. If Nurse Davies is honest, she is glad that she at least has some time to bathe Mr. Johnson today, because some days she has to decide to skip the bed bath. Sometimes, there are too many other important things she needs to take care of.

\section{Daughter Quinn - 57 years old}

Quinn is driving her car to visit her dad for his $87^{\text {th }}$ birthday. Dad has been living in a nursing home for quite some time now and she knows how happy he is every time he sees her. Quinn also likes to visit her father, as she loves him very much. However, lately, he has become a bit grumpy, which takes away a little bit of the pleasure she used to experience when visiting him. Many people will argue that his mood is caused by the recent event of breaking his hip, but it still surprises Quinn because her dad has always been very cheerful. The

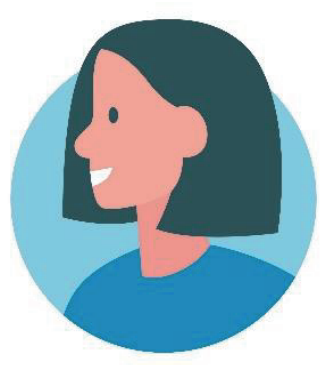

Figure 4 - Daughter Quinn last time that she visited her dad, he complained a lot about the morning care he receives, in particular about the bed bath, which was provided too late. After the last visit, Quinn quickly talked with a nurse - "what was her name again, Nurse Davies!" about his complaints and Nurse Davies suggested to ask her dad for his preferences next time, but that it, unfortunately, will not always be possible to live up to all his requests. Quinn understands that the nurses are very busy and that they do what they can. Still, she wonders if she needs to take more action. She remembers that her dad was bathed with disposable wipes in the hospital right after his hip surgery and wonders if these wipes are also used in the nursing home. Perhaps it is possible to meet 
dad's needs on time with such products without adding to the nurses' workload. Quinn decides that she will discuss this with Nurse Davies after her visit to dad so she can first talk to him about it.

\section{THE BED BATH AS A VALUE CO-CREATION ENCOUNTER IN HEALTH CARE: A SERVICE MARKETING PERSPECTIVE}

In service marketing literature, health care is often presented as a typical transformative service, which is a service that can fundamentally influence wellbeing. ${ }^{14}$ Well-being could relate to the well-being of individual actors (e.g. individual patients) and the well-being of actor networks (e.g. patient-nurse-family triads). Both will be described shortly.

With respect to individual actors, well-being is intertwined with value in that value is 'the change in well-being of a particular actor' (p. 740). ${ }^{15}$ This indicates that actor well-being increases if an actor becomes better off in some sense (i.e. value is created), for example, with respect to quality of life. ${ }^{16,17}$ Whether value is created for an individual actor and his or her well-being subsequently increases depends on the actor's own experiences, which makes value and well-being subjective constructs. ${ }^{18}$ With respect to the bed bath, this implies that actor well-being increases if experiences with the bed bath cause the actor to feel better off. For example, patients might experience the ability to undertake social activities after a bed bath, nurses could experience job satisfaction after providing proper bed baths and observing its uplifting effect on patients, and family members can experience joy in their interactions with patients who are in a good mood because they are properly bathed.

In service marketing, interactions between actors are claimed to affect the value experienced by the different actors, and therefore, their levels of well-being. ${ }^{19}$ For example, with respect to the bed bath, patient well-being can be enhanced when nurses apply their skills to provide bed baths based on professional knowledge and patient preferences. Also, family members can affect value creation, and subsequently, patient well-being; for example, by sharing information with nurses about patients' former bathing preferences, which could be considered during the bed bath. Not only is patient well-being influenced by other actors, but the well-being of nurses and family members can also be affected by the actions of other actors and interactions with other actors.

Actors who are involved in a particular activity, such as the bed bath, are considered to be part of an actor network. If all actors within an actor network get better off, not only do individual actors' well-being increase, but also the well-being of the actor network. Actor network well-being is described in this dissertation as the collective assessments by all network actors of the satisfaction of their individual needs. ${ }^{20}$ When all actors' needs are fulfilled (that is, they are better off resulting in increases in their individual well-being), the actor network's level of well-being increases and balanced centricity is realized. Balanced centricity is a philosophy 
introduced by Gummesson ${ }^{21}$ that stresses the importance of meeting all actors' needs, and describes a desired situation in which all actors' needs are fulfilled simultaneously.22 This dissertation considers the patient-nurse-family network and posits that balanced centricity within the scope of the bed bath exists if all these actors' needs are fulfilled.

In assessing the use of washing without water in health care, this dissertation will focus mostly on its effects on actor experiences, which are crucial for both individual actor well-being and actor network well-being, and reside in the interactions between the different actors within the actor network. ${ }^{23}$ In addition, with respect to the bed bath, actor experiences are dependent on their interactions with other actors; directly, by interactions in the patient-nurse dyad, and indirectly, through the involvement of the family. Health care literature also recognizes that a big part of care recipients' experiences lies in their relationships and interactions with other actors, including nursing staff and family. The health care perspective on the bed bath will be discussed, in which the importance of the bed bath as an essential of care will be initially outlined. Subsequently, relationship-centered care will also be discussed, which underlines the importance of the relationships between actors.

\section{THE BED BATH AS AN ESSENTIAL CARE ACTIVITY: A HEALTH CARE PERSPECTIVE}

Nursing literature recognizes the importance of hygiene care with respect to patient well-being. In the $19^{\text {th }}$ century, hygiene became the hallmark of nursing when Florence Nightingale introduced hygiene assurance as one of nurses' main contributions to patients' health and recovery. ${ }^{24}$ In the $20^{\text {th }}$ century, Virginia Henderson identified personal hygiene as one of the 14 basic nursing components. Personal hygiene is one of the nursing activities that Henderson described as "activities contributing to health, or its recovery (or to a peaceful death) that people would perform unaided if they had the necessary strength, will, or knowledge" (p. 26). ${ }^{25}$ In the $21^{\text {st }}$ century, 'hygiene and personal dressing' is included in the fundamentals of care framework, which stresses its importance as an aspect of care that is fundamental to all patients' health and well-being, regardless of diagnosis, cultural background or health care setting. 26,27

As a means of personal hygiene, the bed bath needs to be recognized for its importance in relation to patient well-being (i.e. particularly, the well-being of bedridden patients).28, 29 Physical well-being is enhanced when the bed bath successfully fulfills purposes, such as removing dirt and sweat, eliminating body odors, reducing infection risks, stimulating blood circulation, and improving skin health. ${ }^{30}$ Furthermore, emotional well-being increases if patients feel refreshed or when the bed bath leads to an enhanced self-image. ${ }^{30,31}$ The interactions that take place during the bed bath could help to build up relationships between patients and nurses, thereby impacting patients' social well-being. As a result, bathing could contribute to physical, emotional, and social well-being, and for that reason, is an essential care activity. ${ }^{32}$ 
Unfortunately, patient experiences with the bed bath are not always positive and the bed bath could negatively affect patient well-being. Bathing can be physically uncomfortable and even painful. Furthermore, many patients feel emotionally affected by being dependent on someone else to maintain their hygiene, violating patients' privacy, dignity or feelings of agency. ${ }^{33}, 34$ Patients who are bathed in bed have long carried out this routinely and straightforward activity themselves, and are now suddenly dependent on others. ${ }^{35}$ Consequently, the patient could experience the bed bath as embarrassing, inhumane, unpleasant, uncomfortable, and unsatisfying. ${ }^{36}$ Such experiences could overshadow the positive effect that the bed bath should have on patients' experiences and well-being.

Due to the potential significant impact of the bed bath on patient well-being (either positive or negative), the bed bath is an important health care activity for nurses as well, and could also impact nurses' well-being. The bed bath offers nurses an opportunity to directly contribute to patient well-being, which is an important clinical goal for nurses. ${ }^{34}$ Furthermore, it can be emotionally rewarding for nurses and beneficial for nurses' well-being to see the contribution they personally make to patient well-being through performance of the bed bath. 25 In addition, the bed bath supports other nursing tasks, such as the assessment of patients' skin conditions and interacting with patients to uncover possible health issues and build trust. ${ }^{37,}, 38$ Hence, the bed bath could support nurses in preventing the deterioration of patients' health. In Henderson's view, the bed bath serves the purpose of supporting patient autonomy and offers patients and nurses the possibility to have intimate interactions with each other. ${ }^{39}$ Such intimate interactions are especially important with respect to older persons with dementia. These persons could show high resistance during the bed bath, which increases the burden on nurses during this time. ${ }^{40}$ The burden of the bed bath on nurses is already quite high due to its labor intensity and physical demands. As a result, the bed bath is also important to nurses' well-being and one might expect that nurses attach high importance to the bed bath.

Unfortunately, the essentials of care (also referred to as 'fundamentals of care') often appear to be undervalued and compromised in health care practice nowadays. ${ }^{27,} 35$ Many nurses attach a low priority to the bed bath and find it uninteresting, especially compared to more complex nursing tasks. ${ }^{13}, 26,29,41$ Some state that, these days nurses are "too posh to wash", 42 and that they tend to underestimate the central importance of the bed bath. Consequently, the bed bath is often routinely provided by lower-skilled nurses. ${ }^{43,44}$ Even more, the bed bath is sometimes omitted due to time constraints, amongst other reasons. ${ }^{45-47}$ For example, a Dutch press release mentioned that bathing is one of the first nursing activities that is compromised during the summer season when nursing capacity is low. ${ }^{48}$ Because the bed bath can take a lot of time, omitting this activity can relieve some pressure. ${ }^{49}$ Hence, "rationing of care" is applicable to the bed bath, which denotes "a failure to offer care, or the denial of care, from which patients would benefit" due to a lack of allocation of (nursing) resources to the bed bath (p. 5).50 
As a result, nurses, regrettably, do not always provide the bed bath to its full potential. The bed bath is an example of a nursing activity that nurses approach instrumentally, based on their routines, over patients' preferences and needs. ${ }^{43,51}$ Consequently, patient autonomy during the bed bath is pressured and the opportunity it offers to strengthen the relationship with the patient is underutilized. This is reinforced, amongst other factors, by work overload and the lack of scientific evidence on the essentials of care that would emphasize its importance and could guide nurses in providing optimal essential care. ${ }^{26,52}$ With respect to the bed bath, evidence regarding patient experiences is lacking, ${ }^{53}$ as well as their experiences with bed bath methods, such as washing without water. ${ }^{54}$

Nurses, as the most intimate helpers of patients, ${ }^{25}$ and also family members, can influence patient experiences with the bed bath, and consequently, their wellbeing. The importance of these actors and the relationships between them is already presented from the service marketing perspective. This importance is underlined in health care literature by the concept of relationship-centered care.

\section{RELATIONSHIP-CENTERED CARE}

Relationship-centered care is based on the premise that quality of care should be considered from the patient's perspective (i.e. patients' experienced quality of care). Furthermore, it posits that patients' experiences of quality of care are largely determined by the interactions that patients have with others, especially staff and family members. Therefore, relationships within the patient-staff-family triad are argued to heavily influence care delivery experiences, 55,56 which is also applicable to the bed bath.

Relationship-centered care builds on patient-centered care, which may contribute to patient and nurse well-being, 52 and is defined as "healthcare that establishes a partnership among practitioners, patients, and their families (when appropriate) to ensure that decisions respect patients' wants, needs, and preferences ..." (p. 7).57 This definition reflects the importance of the patient-stafffamily triad in relation to patient autonomy during health care activities, which could be positively or negatively affected by the actors in the triad. 58

First of all, patients' experiences with the bed bath are likely to be affected by their relationships with nurses. The bed bath is one (sometimes, of the few) moments in which nurses can closely interact with the patient and build trust. ${ }^{59}$ Feo and Kitson state that "meeting another person's intimate care needs is not a simple transactional process; it requires focusing on and connecting meaningfully with the person, understanding their unique needs, and interacting with them in such a way that feelings of embarrassment, frustration and distress are minimized or avoided" (p. 6). ${ }^{27}$ Such an approach would support nurses to integrate patients' needs in care delivery. However, nurses often do not involve patients in decisions about the delivery of daily care, which is mostly provided in a structured way, based on care plans and health care rhythms, instead of patients' daily preferences. 5,58 
Family members often represent another important group of actors that could affect patients' experiences. 60 Family members are argued to be an important source of information for nurses, which supports nurses to deliver optimal care.61 Therefore, family members are often involved in decisions about the care provided to their loved ones to improve health outcomes. ${ }^{62}$ Especially for patients who cannot stand up for themselves when their needs and preferences are not met (e.g. due to cognitive impairments), 63 nurses and family members can heavily influence patient experiences. In some cases, family involvement can be detrimental to patients' experiences when family makes decisions on behalf of patients because they are wrongfully considered to be incapable of making decisions for themselves. ${ }^{64}$

In line with relationship-centered care, this dissertation identifies patients, nurses and family members as the most relevant actors with respect to the bed bath. Nurses and family members could be seen as "secondary customers" of the bed bath, having their own needs, which should be considered in the provision of health care. 65 For example, nurses may have the need for time-efficiency, while family members have the need to feel assured that essential care (including the bed bath) is properly provided. 66 In general, the involvement of patients, nurses, and family is considered to be an important facilitator of improvement.67, 68

\section{DISSERTATION AIM AND OUTLINE}

Based on the literature presented thus far, experiences are recognized to play a central part, in respect to well-being, in both the health care and service marketing streams. Furthermore, both streams underline the importance of the patient-nursefamily triad. This dissertation is all about experiences, that is, health care actors' experiences with the bed bath, and with the washing without water method, that can be applied for this important essential care activity. The aim of this dissertation is to assess the suitability of the washing without water intervention by answering the central research question:

What is the effect of the washing without water intervention on health care actors' experiences and well-being, within the scope of the bed bath?

To assess the effect of washing without water on actors' well-being, actors' experiences with the bed bath and washing without water will be assessed. First, patients', nurses', and family members' experiences with the bed bath, in general, will be explored from the service marketing "value co-creation" perspective in Chapter 2. Both individual actor well-being, as well as actor network well-being, will be studied from a self-determination theory lens, which stresses the importance of the psychological needs of autonomy, competence, and relatedness. Subsequently, Chapters 3-5 will explore the effects of the washing without water intervention on different bed bath outcomes that either represent actors' experiences or could have an impact on actors' experiences, and consequently, well-being. Chapter 6 will 
report on the development and psychometric testing of a tool to measure nurses' beliefs about the importance of the bed bath and the washing without water intervention, which could reflect their experiences. In Chapter 7, the results of the studies that focus on the effectiveness of washing without water will be discussed by relating the findings to the psychological needs of autonomy, competence, and relatedness. Following is a more elaborate outline of the different chapters.

Chapter 2 presents the results of a qualitative study on patients', nurses' and family members' experiences with the bed bath. Based on these actors' rich experiences, the actors' needs related to the bed bath have been explored, which relate to the psychological needs of autonomy, competence, and relatedness. Furthermore, balanced centricity is positioned as an indicator for network well-being, which is realized if all actors' psychological needs are fulfilled. However, tensions between different actors' psychological needs have been found to lead to unbalanced situations in which not all actors' psychological needs are fulfilled simultaneously.

Chapter 3 focuses on the washing without water intervention. It provides an overview of the available evidence on the effectiveness of the washing without water intervention compared to the traditional bed bath with water and soap. This overview is based on a systematic literature review that was conducted in 2017, and includes bed bath outcomes that are mainly relevant to patients (e.g. patient satisfaction) and nurses (e.g. hygienic effectiveness).

Chapter $\mathbf{4}$ offers a detailed description of the study design and methods applied for the study that is presented in Chapter 5.

Chapter 5 reports the outcomes related to comfort in a crossover randomized controlled trial in which nursing students participated as either a patient or nurse. Students that were patients in the trial received two bed baths (one traditional bed bath and one washing without water bed bath) from students that were assigned to be nurses. The differences between emotional and physical comfort were studied from the perspectives of the patient and nurse. Furthermore, nursing students' bed bath method preferences are assessed.

Chapter 6 describes the development and psychometric testing of a tool that measures nurses' beliefs regarding the general importance of the bed bath and the washing without water intervention. This tool is assessed based on data collected in a cross-sectional study and explores nurses' beliefs about the bed bath and the washing without water intervention that could reflect their experiences.

Chapter 7 presents this dissertation's main findings in a general discussion. Furthermore, some theoretical and methodological implications of this dissertation will 


\section{Chapter 1}

be discussed, and suggestions for health care practice and future research are provided in this final chapter.

Table 1 provides an overview of the studies presented in the chapters, and Figure 5 displays how the different studies are related and the actors to whom the results mainly apply. Overall, the results of this dissertation support nurses to provide proper hygiene care to bedridden patients by presenting the importance of the bed bath and results of the washing without water intervention. 12,34,54 


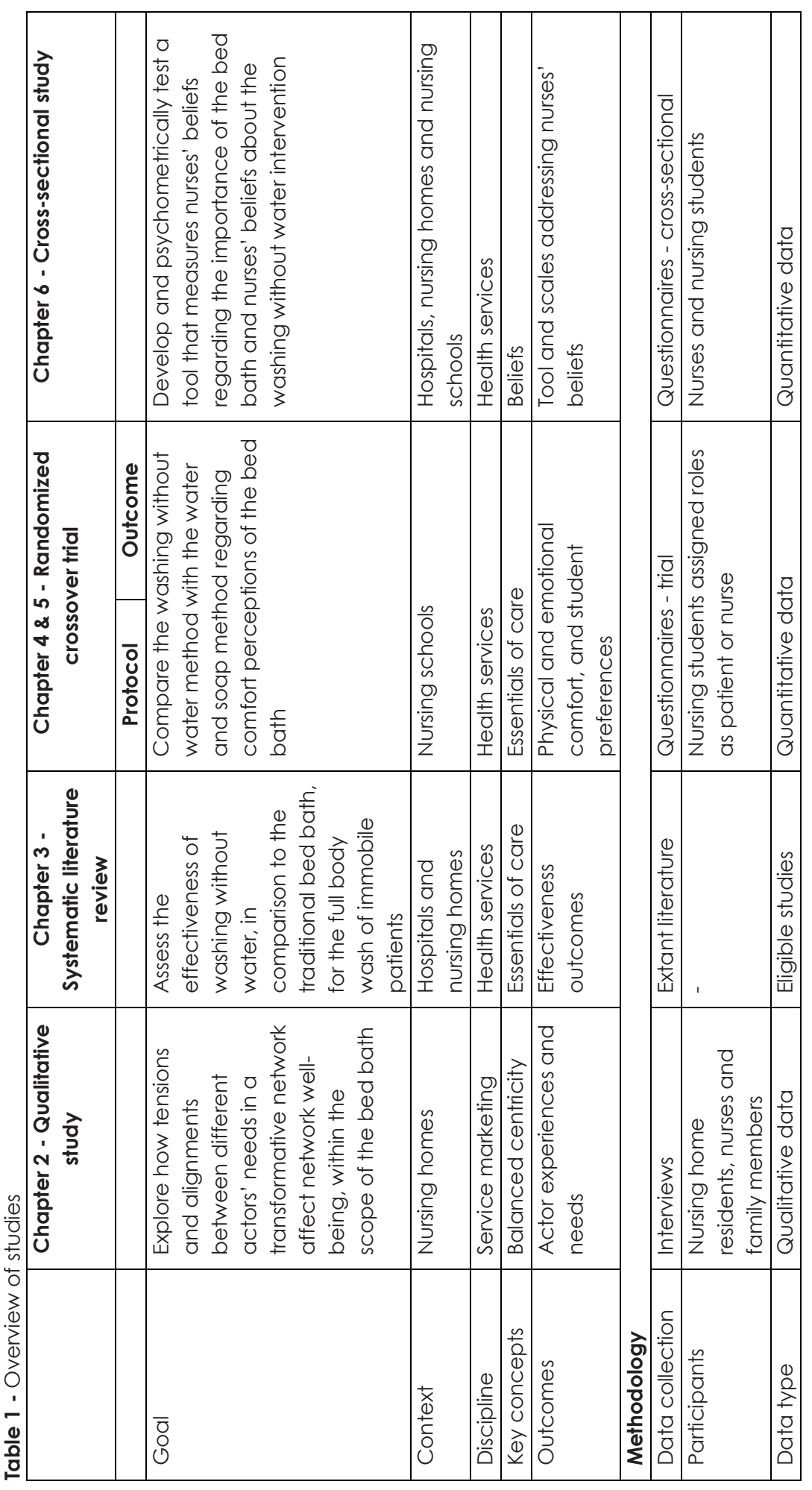




\section{THE BED BATH ACTIVITY IN GENERAL}

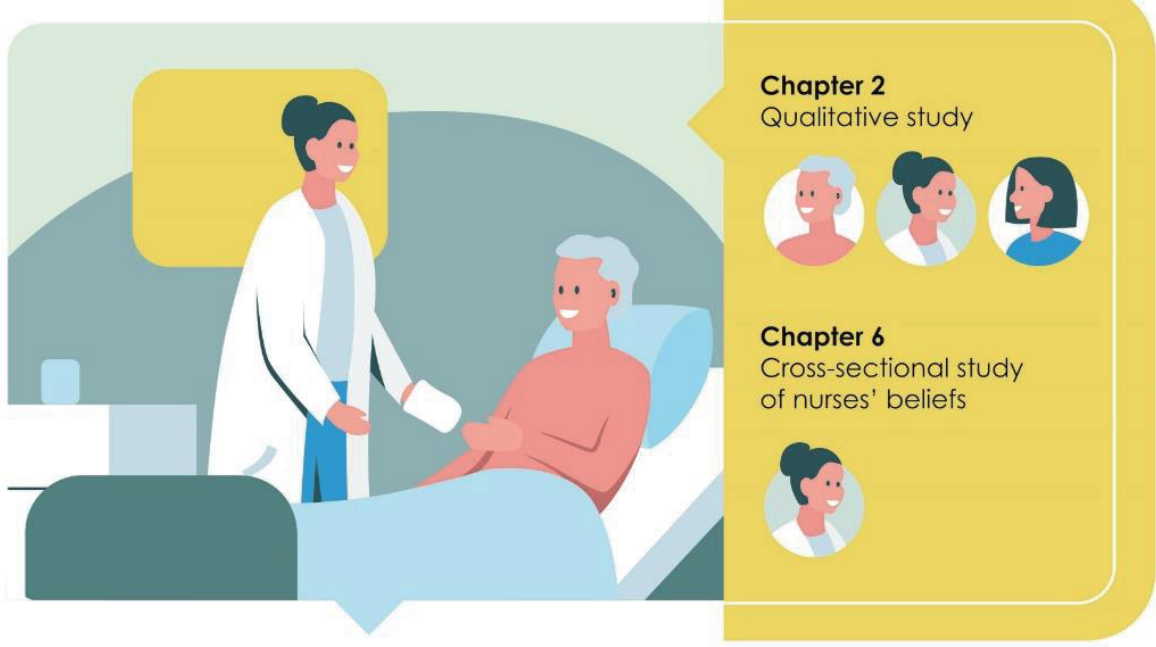

THE BED BATH METHOD

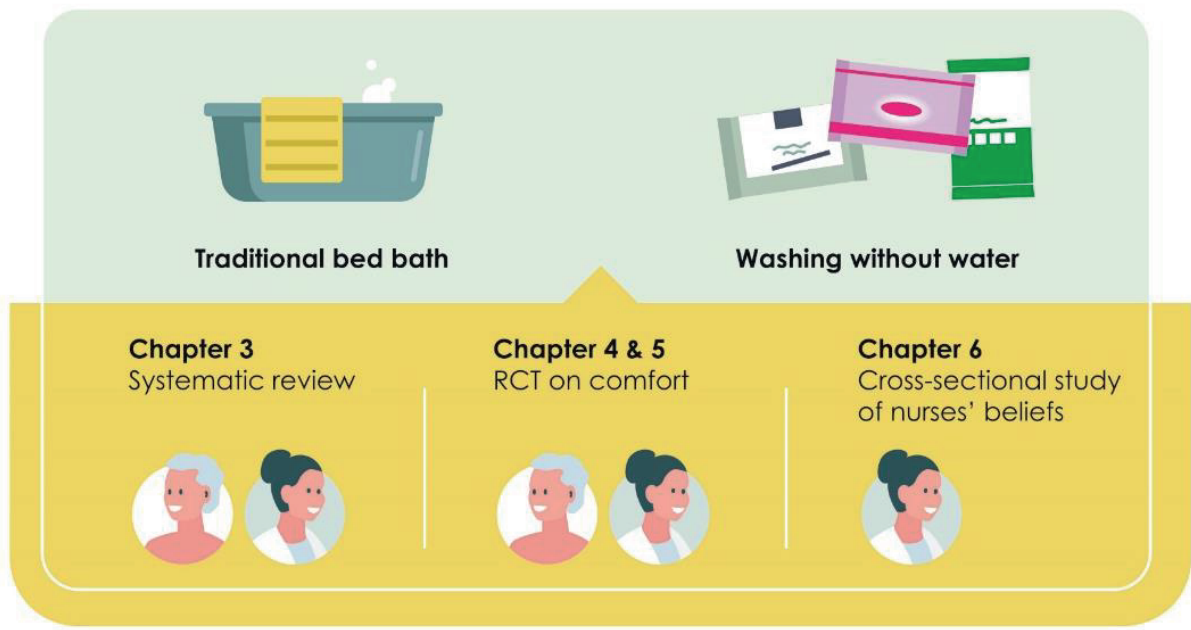

\section{DISCUSSION}

\section{Chapter 7}

Effect of washing without water on actors' well-being

\section{Chapter 4 \& 5}

RCT on comfort

\section{Chapter 6}

Cross-sectional study of nurses' beliefs

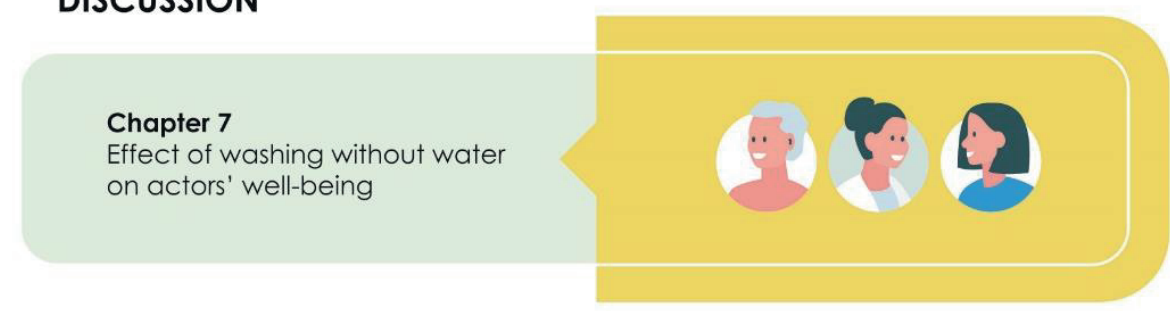

Figure 5 - Visual overview of dissertation chapters 


\section{References}

1. Gill TM, Guo Z and Allore HG. The epidemiology of bathing disability in older persons. Journal of the American Geriatrics Society 2006;54:1524-1530.

2. Naik AD, Concato J and Gill TM. Bathing disability in community-living older persons: common, consequential, and complex. Journal of the American Geriatrics Society 2004;52:1805-1810.

3. Schoonhoven L, van Gaal BGI, Teerenstra S, et al. Cost-consequence analysis of "washing without water" for nursing home residents: a cluster randomized trial. International Journal of Nursing Studies 2015;52:112-120.

4. Jun J, Ojemeni MM, Kalamani R, et al. Relationship between nurse burnout, patient and organizational outcomes: systematic review. International Journal of Nursing Studies 2021.

5. Sherwin $S$ and Winsby M. A relational perspective on autonomy for older adults residing in nursing homes. Health Expectations 2010;14:182-190.

6. Greenhalgh T, Robert G, MacFarlane F, et al. Diffusion of Innovations in Service Organizations: Systematic Review and Recommendations. The Milbank Quarterly 2004;82:581-629.

7. $\quad$ Skewes SM. No more bed baths! RN 1994;57:34-36.

8. Marchaim D, Taylor AR, Hayakawa K, et al. Hospital bath basins are frequently contaminated with multidrug-resistant human pathogens. American Journal of Infection Control 2012;40:562-564.

9. Anaissie EJ, Penzak SR and Dignani MC. The hospital water supply as a source of nosocomial infections. Archives of Internal Medicine 2002;162:1483-1492.

10. Voegeli D. The effect of washing and drying practices on skin barrier function. Journal of Wound, Ostomy and Continence Nursing 2008;35:84-90.

11. Johnson D, Lineweaver L and Maze LM. Patients' bath basins as potential sources of infection: a multicenter sampling study. American journal of critical care 2009; 18: 31-40.

12. Cowdell F and Steventon K. Skin cleansing practices for older people: a systematic review. International Journal of Older People Nursing 2015;10:3-13.

13. Eigsti JE. Innovative solutions: beds, baths, and bottoms: a quality improvement initiative to standardize use of beds, bathing techniques, and skin care in a general critical-care unit. Dimensions of Critical Care Nursing 2011:30:169-176.

14. Ostrom AL, Parasuraman A, Bowen DE, et al. Service Research Priorities in a Rapidly Changing Context. Journal of Service Research 2015;18:127-159.

15. Vargo SL and Lusch RF. The SAGE handbook of service-dominant logic. SAGE Publications Inc. 2018.

16. Grönroos $C$ and Voima P. Critical service logic: making sense of value creation and co-creation. Journal of Academy of Marketing Science 2013;41:133-150.

17. McColl-Kennedy JR, Vargo SL, Dagger TS, et al. Health Care Customer Value Cocreation Practice Styles. Journal of Service Research 2012;15:370-389.

18. Falter $M$ and Hadwich K. Customer service well-being: scale development and validation. The Service Industries Journal 2020;40:181-202.

19. Sweeney JC, Danaher TS and McColl-Kennedy JR. Customer Effort in Value Cocreation: Improving Quality of Life and Behavioral Intentions of Health Care Customers. Journal of Service Research 2015;18:318-335. 
20. Leo WWC, Laud G and Chou CY. Service system well-being: conceptualising a holistic concept. Journal of Service Management 2019;30:766-792.

21. Gummesson E. Exit services marketing - enter service marketing. Journal of Customer Behaviour 2007:6:113-141.

22. Verleye K, Jaakkola E, Hodgkinson IR, et al. What causes imbalance in complex service networks? Evidence from a public health service. Journal of Service Management 2017;28:34-56.

23. Pinho N, Beirão G, Patrício $L$, et al. Understanding value co-creation in complex services with many actors. Journal of Service Management 2014;25:470-493.

24. Matthews JH, Whitehead PB, Ward C, et al. Florence Nightingale: Visionary for the Role of Clinical Nurse Specialist. The Online Journal of Issues in Nursing 2020;25:1-9.

25. Henderson $\vee$. The concept of nursing. Journal of Advanced Nursing 1978;3:1 13130.

26. Zwakhalen SMG, Hamers JP, Metzelthin SF, et al. Basic nursing care: the most provided, the least evidence based. A discussion paper. Journal of Clinical Nursings 2018;27:2496-2505.

27. Feo $\mathrm{R}$ and Kitson A. Promoting patient-centred fundamental care in acute healthcare systems. International Journal of Nursing Studies 2016:57:1-11.

28. Jangland E, Mirza N, Conroy T, et al. Nursing students' understanding of the Fundamentals of Care: A cross-sectional study in five countries. Journal of Clinical Nursing 2018;27:2460 - 2472.

29. Feo R, Frensham LJ, Conroy T, et al. "It's just common sense": Preconceptions and myths regarding fundamental care. Nurse Education in Practice 2019:36:82-84.

30. Sheppard CM and Brenner PS. The effects of bathing and skin care practices on skin quality and satisfaction with an innovative product. Journal of Gerontological Nursing 2000;26:36-45.

31. Ahluwalia SC, Gill TM, Baker DI, et al. Perspectives of older persons on bathing and bathing disability: a qualitative study. Journal of the American Geriatrics Society 2010;58:450-456.

32. Webster R, Thompson DR, Bowman G, et al. Patients' and nurses' opinions about bathing. Nursing Times 1988;84:54-57.

33. Evans LK. The bath! Reassessing a familiar elixir in old age. Journal of the American Geriatrics Society 2004:52:1957-1958.

34. Downey L and Lloyd H. Bed bathing patients in hospital. Nursing Standard 2008;22:35-40.

35. Kitson A, Conroy T, Kuluski K, et al. Reclaiming and redefining the Fundamentals of Care: Nursing's response to meeting patients' basic human needs. Adelaide, South Australia: School of Nursing, The University of Adelaide 2013.

36. Lopes JL, Nogueira-Martins LA and de Barros AL. Bed and shower baths: comparing the perceptions of patients with acute myocardial infarction. Journal of Clinical Nursing 2012;22:733-740.

37. Coyer FM, O'Sullivan J and Cadman N. The provision of patient personal hygiene in the intensive care unit: A descriptive exploratory study of bed-bathing practice. Australian Critical Care 2011;24:198-209.

38. Kottner J, Hahnel E, Trojahn C, et al. A multi-center prevalence study and randomized controlled parallel-group pragmatic trial to compare the effectiveness of standardized skin care regimens on skin health in nursing home 
residents: A study protocol. International Journal of Nursing Studies 2015;52:598604.

39. Boge J, Kristoffersen $\mathrm{K}$ and Martinsen K. Bodily cleanliness in modern nursing. Nursing Philosophy 2013;14:78-85.

40. Hoeffer B, Talerico KA, Rasin J, et al. Assisting cognitively impaired nursing home residents with bathing: effects of two bathing interventions on caregiving. The Gerontologist 2006;46:524-532.

41. Vollman K, Garcia R and Miller L. Interventional patient hygiene: proactive (hygiene) strategies to improve patients' outcomes. AACN News 2005;22:12-16.

42. Moore KN. Does "arriving" mean we give up the bed bath? Journal of Wound Ostomy and Continence Nursing 2005;32:285-286.

43. Koehn ML and Lehman K. Nurses' perceptions of evidence-based nursing practice. Journal of Advanced Nursing 2008;62:209-215.

44. Richards DA and Borglin G. 'Shitty Nursing' - the new normal? International Journal of Nursing Studies 2019:91:148-152.

45. Parr JM, Bell J and Koziol-McLain J. Evaluating fundamentals of care: The development of a unit-level quality measurement and improvement programme. Journal of Clinical Nursing 2018;27:2360-2372.

46. Pipe TB, Connolly T, Spahr N, et al. Bringing Back the Basics of Nursing: defining patient care essentials. Nursing Admininstration Quarterly 2012;36:225-233.

47. Feo R, Kitson A and Conroy $\mathrm{T}$. How fundamental aspects of nursing care are defined in the literature: A scoping review. Journal of Clinical Nursing 2018;27:21892229.

48. Verpleegkundigen en verzorgenden deze zomer oververhit en onderbezet. Verpleegkundigen \& Verzorgenden Nederland. 2019. Available from: https://www.venvn.nl/nieuws/verpleegkundigen-en-verzorgenden-deze-zomer-

oververhit-en-onderbezet/. Accessed 31 Jul 2019.

49. Dahlke SA, Phinney A, Hall WA, et al. Orchestrating care: nursing practice with hospitalised older adults. International Journal of Older People Nursing 2014;10:252-262.

50. Maynard A. Rationing health care: an exploration. Health Policy 1999;49:5-11.

51. El-Soussi AH and Asfour HI. Examining bed-bath practices of critically ill patients. Journal of Nursing Education and Practice 2016;12:1-11.

52. Grilo AM, Santos MC, Rita JS, et al. Assessment of nursing students and nurses' orientation towards patient-centeredness. Nurse Education Today 2014;34:35-39.

53. Richards DA, Hilli A, Pentecost C, et al. Fundamental nursing care: A systematic review of the evidence on the effect of nursing care interventions for nutrition, elimination, mobility and hygiene. Journal of Clinical Nursing 2018;27:2179-2188.

54. Thoma-Lürken T, Bleijlevens MH, Lexis MA, et al. An overview of potential laborsaving and quality-improving innovations in long-term care for older people. Journal of the American Medical Directors Association 2015;16:482-489.

55. Sion $\mathrm{KYJ}$, Verbeek $\mathrm{H}$, de Boer B, et al. How to assess experienced quality of care in nursing homes from the client's perspective: results of a qualitative study. BMC Geriatrics 2020;20:1-12.

56. Sion KYJ, Haex R, Verbeek H, et al. Experienced Quality of Post-Acute and LongTerm Care From the Care Recipient's Perspective-A Conceptual Framework. Journal of the American Medical Directors Association 2019;20:1386-1390. 
57. Crossing the Quality Chasm: A new health system for the 21 st century. Institute of Medicine. 2001. Available from: https://iom.nationalacademies.org/ /media/Files/Report\%20Files/2001/Crossingthe-Quality-Chasm/Quality\%20Chasm\%202001\%20\%20report\%20brief.pdf. Accessed 26 Nov 2015.

58. Simmons SF, Rahman A, Beuscher L, et al. Resident-Directed Long-Term Care: Staff Provision of Choice During Morning Care. The Gerontologist 201 1;51:867-875.

59. Powers J and Fortney S. Bed baths: much more than a basic nursing task. Nursing 2014:44:67-68.

60. McColl-Kennedy JR, Danaher TS, Gallan AS, et al. How do you feel today? Managing patient emotions during health care experiences to enhance wellbeing. Journal of Business Research 2017:79:247-259.

61. Frow P, McColl-Kennedy JR and Payne A. Co-creation practices: Their role in shaping a health care ecosystem. Industrial Marketing Management 2016;56:2439.

62. Berwick DM. What 'Patient-Centered' Should Mean: Confessions Of An Extremist. Health Affairs 2009;28:w555-w565.

63. Bangerter LR, Heid AR, Abbott K, et al. Honoring the Everyday Preferences of Nursing Home Residents: Perceived Choice and Satisfaction With Care. The Gerontologist 2017; 57:479-486.

64. McCormack B. Autonomy and the relationship between nurses and older people. Ageing and Society 2001;21:417-446.

65. Leino HM. Secondary but significant: secondary customers' existence, vulnerability and needs in care services. Journal of Services Marketing 2017:31:760-770.

66. Hoof van J, Verbeek $\mathrm{H}$, Janssen BM, et al. A three perspective study of the sense of home of nursing home residents: the views of residents, care professionals and relatives. BMC Geriatrics 2016;16:1-15.

67. Berry LL. Service innovation is urgent in healthcare. AMS Review 2019:9:78-92.

68. Cramm JM and Nieboer AP. Validation of an instrument to assess the delivery of patient-centred care to people with intellectual disabilities as perceived by professionals. BMC Health Services Research 2017;17:1-11. 


\title{
chapter
}

\section{NETWORK WELLBEING FROM A BALANCED CENTRICITY PERSPECTIVE}

\author{
This chapter was published as: \\ Groven F, Odekerken-Schrö der G. \\ Zwakhalen S, and Hamers $J$. Network \\ well-being from a balanced centricity \\ perspective. Journal of Services \\ Marketing 2021,35:1-14 \\ doi.org110.1108/JSM-11-2020-0466
}




\section{ABSTRACT}

Purpose: This paper explores how tensions and alignments between different actors' needs in a transformative services network affect balanced centricity, which is an indicator of well-being. Balanced centricity describes a situation in which all network actors' interests and needs are fulfilled simultaneously. In such cases, all actors are better off, which increases both individual actors' and overall actor network wellbeing.

Design/methodology/approach: The empirical study takes place in nursing homes in which bed baths represent co-created service encounters that affect the well-being of focal actors (i.e. patients), frontline service employees (i.e. nurses), and transformative service mediators (i.e. family members), who have potentially competing needs. Using a qualitative, phenomenological approach, the study inductively explores and deductively categorizes actors' personal experiences to gain deep, holistic insights into the service network and its complex web of actor interdependencies.

Findings: The resulting conceptual model of balanced centricity identifies actors' lower-order needs as different manifestations of the psychological needs for autonomy, competence, and relatedness. If actors' needs are aligned, their psychological needs can be satisfied, which facilitates balanced centricity. If actors exhibit competing needs though, balanced centricity is impeded.

Practical implications: This study establishes actors' psychological needs as the origin of tensions/alignments in multi-actor networks that impede/contribute to balanced centricity. Transformative service providers should try to address all actors' psychological needs when co-creating services to achieve network well-being.

Originality: This study adopts a novel, multi-actor perspective and thereby presents a conceptual model that contributes to the understanding of balanced centricity. Future research could test this model in other transformative service settings.

\section{Acknowledgments}

The authors gratefully acknowledge the support of the managers from the participating health care institutions as well as the study's participants for their willingness to share their personal experiences. 


\section{INTRODUCTION}

Well-being is a central consideration for transformative service research (TSR) scholars and practitioners, especially as it relates to vulnerable populations. ${ }^{1,2}$ Vulnerability refers to actors' inability to fulfill their needs because of a lack of power or control. ${ }^{3}$ Health care, as an example of a TSR context highly relevant to societal and individual well-being, can enhance the well-being of vulnerable patient populations and workers, though such outcomes often are challenging and health care systems are in distress. ${ }^{4}, 5$ As Verleye et al. illustrate, imbalances, or situations in which "legitimate interests of at least one actor in a complex service network are not secured", have detrimental effects on patients' well-being and the stability of health care systems overall (p. 35). ${ }^{6}$ Imbalances can arise from unintended consequences of wellintentioned health care developments. For example, the focus on productivity in health care encourages health care staff to serve as many patients as possible, at the expense of patients' need to build trusted relationships with the staff. ${ }^{7}$ Imbalances also might affect other involved actors, such as family members. ${ }^{8}$

Within service industries, such as health care, actors make up multi-actor networks in which their interactions affect other actors' individual well-being as well as the collective actor-network well-being . 9, 10 The current study adopts Leo et al.'s definition of service system well-being to define actor-network well-being as "the aggregate perception of actor assessments of the system in terms of the fulfilment of their collective, and by implication, the satisfaction of their individual needs" ( $p$. 782). ${ }^{11}$ Balanced centricity, a concept introduced by Gummesson, emphasizes the importance of satisfying all actors' needs. ${ }^{12}$ The current study describes a situation in which all network actors' interests and needs are fulfilled simultaneously as 'balanced centricity'.6, 13

Actors' interactions that affect individual actor and collective actor-network well-being are part of co-creation of services. With the term 'co-creation of services', we refer specifically to the integration of resources in collaborative activities by frontline service employees (FLES) (e.g. nurses) and focal actors (e.g. patients), influenced by transformative service mediators (TSMs; e.g. family members), that can result in beneficial outcomes and increase actor well-being. ${ }^{14}$ TSMs are actors who are involved in co-creation of services as mediators to support vulnerable focal actors, especially focal actors who are too incapacitated to co-create by themselves (e.g. anxious patients who are not confident enough to question and address health care staff's decisions).' According to Grönroos and Voima, 15 actor well-being improves when the actor is better off as a result of co-creation of services, whether with respect to quality of life, health, happiness, or some other measure. ${ }^{6}$ Such a view of actor well-being is subjective, such that each actor's evaluation is based on her or his personal experiences. ${ }^{17}$

We acknowledge the value co-creation logics' reasoning, ${ }^{15}, 18$ in which (transformative) value and well-being are inherently intertwined, ${ }^{19}$ and value is "the change in well-being of a particular actor" (p. 740)..$^{20}$ Co-creation of services affects the well-being of all interrelated actors, so one actor's activities in co-creation of 


\section{Chapter 2}

services affect not only his or her individual well-being but also the well-being of other actors related to him or her. ${ }^{21}$ When co-creation of services fulfills all actors' interests and needs (i.e. balanced centricity exists) and therefore creates beneficial outcomes for all actors involved, all actors' well-being increases as well as the multi-actor network well-being.

Therefore, we propose that balanced centricity offers an indicator of multiactor network well-being. That is, when all actors' interests and needs are being met and beneficial outcomes are created for all actors, all the actors become better off, which means their individual-level well-being increases. Furthermore, actors perceive the network as able to fulfill their needs, so multi-actor network well-being increases as well, along with individual actors' well-being. ${ }^{11}$

However, co-creation of services can result in counterproductive outcomes if tensions exist among the varied interests and needs of different actors. ${ }^{14,} 22$ If the actors' interests and needs are contradictory, co-creation of services could contribute to the individual well-being of one actor while degrading other, interrelated actors' well-being. ${ }^{19,23}$ Especially in complex services such as health care, unintended effects on actors' needs could occur. ${ }^{7}$ For example, a tension may exist between the need of immobile patients (i.e. focal actors), who cannot bathe independently, to be bathed on a daily basis to maintain their dignity and the need of nurses (i.e. FLEs) to provide this service thoroughly and considerately, such that they cannot provide daily bed baths to all patients, given the time constraints involved in their work. Network well-being does not depend solely on a single, recurring activity, such as bed baths, but it could represent other health care encounters and therefore offers a clear illustration of the potential impact of tensions in different actors' needs on the actors' and, ultimately, network well-being.

In extant literature, the reasons that tensions exist remain unclear. By taking a multi-actor perspective, we seek to explore the origins of both tensions and alignments across the interests and needs of different actors, as well as how they affect network well-being, in accordance with the following research questions:

1. What are actors' (different) interests and needs related to the bed bath as a representation of co-created service encounters?

2. From where do tensions and alignments in interests and needs across actors originate?

3. How do tensions and alignments across different actors' interests and needs affect individual actor well-being, and how do they affect the achievement of balanced centricity as an indicator of network wellbeing?

In turn, this study contributes to services marketing literature in three ways. First, it responds to Sweeney et al.'s call for multi-actor approaches and employs a qualitative, empirical research approach to explore the interests and needs expressed in firsthand health care experiences described by vulnerable focal actors, FLEs, and TSMs. ${ }^{21}$ By exploring multiple actors' experiences, we build on research that explores network influences on focal actors' experiences exclusively. ${ }^{24,}{ }^{25}$ Second, our 
empirical study adds to largely conceptual literature on balanced centricity; we introduce a conceptual model that can identify tensions and alignments in interests and needs across actors that impede or contribute to balanced centricity (i.e. satisfaction of all actors' interests and needs). By postulating balanced centricity as an indicator of multi-actor network well-being, we establish network well-being as an actionable, less abstract concept in response to calls to examine well-being at the network level, in addition to individual actor well-being. ${ }^{7}$ Third, the conceptual model reveals the origin of tensions and alignments across different actors' interests and needs. Previous studies detail what activities health care actors undertake in the cocreation of services and how these activities might affect (other) actors' well-being. ${ }^{16}$, 21,26 By focusing on the origin of actors' interests and needs-or why they take part in the co-creation of services-with respect to network well-being, we provide valuable recommendations for how service managers can address all actors' interests and needs simultaneously and thereby facilitate balanced networks.

\section{THEORETICAL BACKGROUND}

\section{Balanced centricity and network well-being}

This study addresses the well-being of networks that comprise multiple actors, with the notion that various actors' well-being must be considered in health care. ${ }^{10}$ To describe multi-actor networks, we turn to definitions of value networks as "service beneficiaries' conceptualizations of actor constellations and their value co-creating/destroying dynamics" (p. 180). ${ }^{27}$ Our definition of network well-being implies that network wellbeing depends on the well-being of its individual actors. ${ }^{11,28}$ As noted, individual actor well-being in this study refers to actors' subjective well-being, which is based on actors' experiences.

We anticipate that individual actors' well-being increases if co-creation of services leaves them better off, such that they perceive some improvement, based on their personal experiences. ${ }^{15,} 17$ Individual actors integrate resources through cocreation of services, which results in their well-being if it generates beneficial outcomes for them. 14, 15, 29 An actor's level of well-being depends on both his or her own and others' activities in co-creation of services.30 Particularly for vulnerable focal actors, such as impaired patients, well-being is affected by their relationships with FLES and TSMs (e.g. family supporting patients in co-creation of services).

Such interrelationships between actors and their respective resources necessitate a holistic view of network well-being, as a complex phenomenon. We propose balanced centricity as an indicator to simplify this complexity. " Gummesson introduced the concept of balanced centricity as a marketing philosophy that stresses the importance for organizations to meet all actors' interests and needs, instead of adopting only a customer-centric approach. ${ }^{2}$, 31 Rather than solely a philosophy, we investigate balanced centricity as a system-level outcome, reached only if all actors' interests and needs are satisfied. 12, 13, 32 
The satisfaction of actors' interests and needs is a beneficial outcome of cocreation of services that increases actors' individual well-being; service co-creation can also increase network well-being, depending on the embedded actors' assessments of the network's capacity to fulfill their needs. ${ }^{11}$ The opposite of balanced centricity is an imbalanced network, which results if co-creation of services generates beneficial outcomes for one actor and counterproductive outcomes for other, interrelated actors. ${ }^{33}$ In health care services, examples of counterproductive outcomes include an excessive burden on FLEs (e.g. due to high focal actors' demands), dissatisfied TSMs (e.g. due to focal actors' criticism), or negative emotions among focal actors, such as feelings of dependence or indignity (e.g. due to FLEs' unavailability). Beneficial outcomes must accrue to all actors to remain within a balance threshold and avoid surpassing it, which would diminish overall network wellbeing. 6,22

\section{Tensions and alignments in interests and needs across actors}

Interests and needs determine actors' activities in co-creation of services. 34,35 Actors' motives for ensuring their needs define the resource integration process and give direction to co-creation. ${ }^{36}$ When interests and needs across actors are asymmetric, the result might be co-destruction and "a decline in at least one of the systems' (e.g. actors) well-being" (p. 431).23

Extant literature on balanced centricity largely consists of conceptual studies, while empirical studies are largely lacking. Even the few empirical studies of cocreation in networks that take multiple actors' perspectives tend to ignore the tensions and alignments in interests and needs across actors in relation to network (im)balances. Verleye et al. consider network imbalances from a patient (i.e. focal actor) perspective and conclude that imbalances originate from competing logics (e.g. patient care vs. business logics). ${ }^{6}$ For example, they observed patients being left unwashed (their basic care needs left unmet) as a result of the health care organization's focus on efficiency (to meet their business needs). We build on their work by empirically zooming in on the effect of tensions and alignments between the interests and needs of different actors from the perspective of three interrelated actors in a health care setting, to understand the sources of their interests and needs, and thereby explore whether balanced centricity is attainable. ${ }^{21}$

By empirically exploring tensions and alignments in interests and needs across different health care actors, we also aim to enhance understanding of the interrelationships between actor and network well-being in a transformative service setting. ${ }^{35}$ Transformative service settings that feature vulnerable actors generally create a higher risk of tensions among network actors. ${ }^{37}$ As a transformative setting, for this study we address the recurring service encounter of bed baths, which usually are dyadic health care activities that require the direct involvement of vulnerable patients being bathed in bed and nurses providing the bed bath. Yet this service cocreation also is influenced by a multi-actor network, 14 such that the setting also includes the patient's family members, who are involved mostly indirectly (i.e. as 
informal caregivers and TSMs). Still, their interests and needs need to be fulfilled to achieve balanced centricity, in which all actors' interests and needs are satisfied. The bed bath is an important daily health care encounter that is representative of other health care encounters in which tensions and alignments between actors come to the surface.

\section{METHODOLOGY}

\section{Setting and approach}

Health care is an appropriate setting to explore the effect of tensions and alignments across actors' interests and needs on multi-actor network well-being, due to its high level of complexity and the presence of many actors, actions, and interactions, even within single health care encounters. ${ }^{38}$ Furthermore, health care is a typical transformative service that has a significant impact on vulnerable actors with physical and/or cognitive impairment. We investigate bed baths because they represent transformative health care encounters that affect the well-being of multiple actors, including vulnerable focal actors, with potentially competing interests. Bedridden patients (i.e. the focal actors) who cannot bathe independently feel embarrassed and worried about their dignity, and the bed baths, as recurring service encounters, can have significant impacts on their quality of life and well-being. 39,40 Nurses and family may be affected by this service encounter in different ways. Nurses (i.e. FLEs) perceive bed bathing as a relatively straightforward, daily routine, with little impact on their personal well-being. ${ }^{41}$ Patients' family members (i.e. TSMs) likely view such personal hygiene encounters as determinants of the quality of care provided to their loved ones. These different actor perceptions could hint at differences in actors' interests and needs with regard to bed baths, as also might exist in other daily health care encounters, such as feeding, dressing, and toilet assistance.

The data collection took place in nursing homes in a Western European country. Descriptions of bed bathing experiences were collected inductively from three actor groups within this setting: patients, nurses, and patients' family members. Their experiences reflect various bathing methods across four nursing homes from which we gathered data. In one, patients are bathed exclusively in bed with water and soap (i.e. traditional bed bath), but three nursing homes also use a washingwithout-water concept, relying on disposable, prepacked bathing systems. The nursing homes were affiliated with different institutions, but they provided care to patients with comparable physical and cognitive impairments.

With a qualitative approach, we inductively explored the actors' service experiences to gain deep, holistic insights into the network and its complex web of actor interdependencies. ${ }^{42}$ We sought actors' personal interpretations of the bed bath, because co-creation is experience-centric. ${ }^{14}$ Therefore, we adopted an interpretive, phenomenological stance to explore the meanings that different actors ascribe to their experiences with bed baths. ${ }^{43}$ 


\section{Data collection}

We collected experiences from different actors to draw conclusions about multi-actor networks, as the unit of analysis. The units of observation were individual patients (focal actors), nurses (FLEs), and family members (TSMS) within networks. The patients we interviewed expressed minimal cognitive impairments but moderate to high physical impairments. Managers of the participating nursing homes selected eight patients on their (cognitive) ability to share their bed bath experiences; all agreed to participate. The aim was to interview at least one nurse and one family member for each patient included. However, for some patients, family members were not available. For each patient, at least one nurse was interviewed who had bathed him or her, and for five patients, we also interviewed a family member. Thus, in total, we conducted 19 indepth interviews with eight patients, six nurses, and five family members (Table 1), resulting in five triads and three patient-nurse dyads (Figure 1) that are part of multiactor networks. We included data from the patient-nurse dyads because they provide direct information about patients' and nurses' experiences, as well as indirect information about the family's experiences. By interviewing health care actors directly related to one another, we also achieved data triangulation and increased the validity and reliability of the findings. ${ }^{44}$ No new information emerged from the final interviews, so we perceived that data saturation had been achieved. 45

Table 1 - Sample

\begin{tabular}{|c|c|c|c|c|c|c|}
\hline Actor & $\begin{array}{l}\text { Nursing } \\
\text { home }\end{array}$ & Gender & Age & $\begin{array}{l}\text { Educational } \\
\text { level }\end{array}$ & $\begin{array}{l}\text { Bathing } \\
\text { method(s) }\end{array}$ & $\begin{array}{l}\text { Interview } \\
\text { length in } \\
\text { minutes }\end{array}$ \\
\hline \multicolumn{7}{|l|}{ Actor network 1} \\
\hline Patient 1 (Mrs. Smith) & 1 & Female & 95 & $\begin{array}{l}\text { Primary } \\
\text { school }\end{array}$ & $\begin{array}{l}\text { Water } \\
\text { and soap }\end{array}$ & 66 \\
\hline Nurse 1 (James) & & Male & 28 & $\begin{array}{l}\text { Intermediate } \\
\text { nursing }\end{array}$ & & 55 \\
\hline Family 1 (son Walter) & & Male & 67 & University & & 42 \\
\hline \multicolumn{7}{|l|}{ Actor network 2} \\
\hline Patient 2 (Mrs. Green) & 1 & Female & 81 & $\begin{array}{l}\text { Primary } \\
\text { school }\end{array}$ & $\begin{array}{l}\text { Water } \\
\text { and soap }\end{array}$ & 45 \\
\hline Nurse 1 (James) & & Male & 28 & $\begin{array}{l}\text { Intermediate } \\
\text { nursing }\end{array}$ & & 55 \\
\hline \multicolumn{7}{|l|}{ Actor network 3} \\
\hline Patient 3 (Mrs. Brown) & 1 & Female & 87 & $\begin{array}{l}\text { Primary } \\
\text { school }\end{array}$ & $\begin{array}{l}\text { Water } \\
\text { and soap }\end{array}$ & 57 \\
\hline Nurse 2 (Fred) & & Male & 40 & $\begin{array}{l}\text { Intermediate } \\
\text { nursing }\end{array}$ & & 48 \\
\hline $\begin{array}{l}\text { Family } 3 \text { (daughter } \\
\text { Bessie) }\end{array}$ & & Female & 60 & $\begin{array}{l}\text { Primary } \\
\text { school }\end{array}$ & & 52 \\
\hline
\end{tabular}




\begin{tabular}{|c|c|c|c|c|c|c|}
\hline Actor & $\begin{array}{l}\text { Nursing } \\
\text { home }\end{array}$ & Gender & Age & $\begin{array}{l}\text { Educational } \\
\text { level }\end{array}$ & $\begin{array}{l}\text { Bathing } \\
\text { method(s) }\end{array}$ & $\begin{array}{l}\text { Interview } \\
\text { length in } \\
\text { minutes }\end{array}$ \\
\hline \multicolumn{7}{|l|}{ Actor network 4} \\
\hline Patient 4 (Mrs. Wilson) & 1 & Female & 92 & $\begin{array}{l}\text { Primary } \\
\text { school }\end{array}$ & $\begin{array}{l}\text { Water } \\
\text { and soap }\end{array}$ & 35 \\
\hline Nurse 2 (Fred) & & Male & 40 & $\begin{array}{l}\text { Intermediate } \\
\text { nursing }\end{array}$ & & 48 \\
\hline Family 4 (son Edward) & & Male & 63 & University & & 33 \\
\hline \multicolumn{7}{|l|}{ Actor network 5} \\
\hline Patient 5 (Mr. Taylor) & 2 & Male & 80 & $\begin{array}{l}\text { Secondary } \\
\text { school }\end{array}$ & $\begin{array}{l}\text { Water } \\
\text { and soap }\end{array}$ & 57 \\
\hline Nurse 5 (Kim) & & Female & 23 & $\begin{array}{l}\text { Advanced } \\
\text { nursing }\end{array}$ & $\begin{array}{l}\text { and } \\
\text { washing } \\
\text { without } \\
\text { water }\end{array}$ & 51 \\
\hline \multicolumn{7}{|l|}{ Actor network 6} \\
\hline Patient 6 (Mr. Thomas) & 3 & Male & 60 & Intermediate & Water & 41 \\
\hline Nurse 6 (Florence) & & Female & 52 & $\begin{array}{l}\text { Intermediate } \\
\text { nursing }\end{array}$ & $\begin{array}{l}\text { and soap } \\
\text { and }\end{array}$ & 48 \\
\hline $\begin{array}{l}\text { Family } 6 \text { (relative } \\
\text { Minnie) }\end{array}$ & & Female & 56 & $\begin{array}{l}\text { Applied } \\
\text { university }\end{array}$ & $\begin{array}{l}\text { washing } \\
\text { without } \\
\text { water }\end{array}$ & 30 \\
\hline \multicolumn{7}{|l|}{ Actor network 7} \\
\hline Patient 7 (Mrs. Harris) & 3 & Female & 72 & $\begin{array}{l}\text { Secondary } \\
\text { school }\end{array}$ & $\begin{array}{l}\text { Water } \\
\text { and soap }\end{array}$ & 42 \\
\hline Nurse 7 (Alice) & & Female & 32 & $\begin{array}{l}\text { Intermediate } \\
\text { nursing }\end{array}$ & $\begin{array}{l}\text { and } \\
\text { washing }\end{array}$ & 40 \\
\hline $\begin{array}{l}\text { Family } 7 \text { (husband } \\
\text { Frank) }\end{array}$ & & Male & 73 & $\begin{array}{l}\text { Applied } \\
\text { university }\end{array}$ & $\begin{array}{l}\text { without } \\
\text { water }\end{array}$ & 30 \\
\hline \multicolumn{7}{|l|}{ Actor network 8} \\
\hline Patient 8 (Mr. Robinson) & 4 & Male & 72 & Intermediate & Water & 32 \\
\hline Nurse 8 (Polly) & & Female & 46 & $\begin{array}{l}\text { Intermediate } \\
\text { nursing }\end{array}$ & $\begin{array}{l}\text { and soap } \\
\text { and } \\
\text { washing } \\
\text { without } \\
\text { water }\end{array}$ & 47 \\
\hline
\end{tabular}




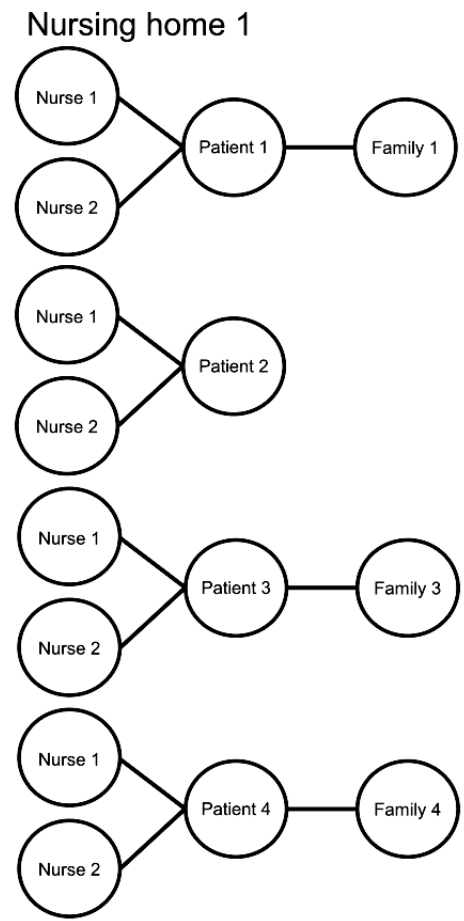

Nursing home 2

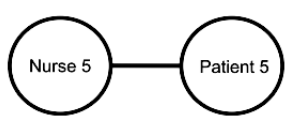

Nursing home 3

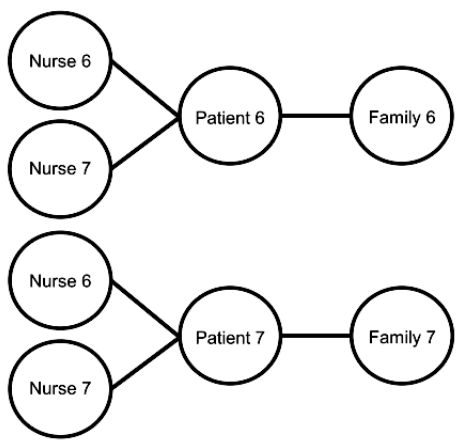

Nursing home 4

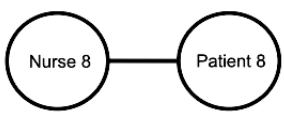

Figure 1 - Multi-actor networks

The interview scripts varied for the interviews with patients, nurses, and family, in which we inductively (i.e. without theoretical preconceptions) collected actors' general experiences with, as well as their interests and needs related to, the bed bath (see appendix 1). Open questions helped encourage the interviewees to elaborate on their experiences in their own words, without imposing the researchers' ideas. ${ }^{46}$ Furthermore, follow-up questions sought to identify possible socially desirable answers. The first author transcribed all interviews verbatim within a week; none of the interviews was coded or analyzed before all the other interviews had been conducted.

\section{Data analysis}

The data analysis process shown in Figure 2 consisted of several inductive steps at the start and deductive steps at the end of the process that allowed the codes to evolve across data and theory. The first author read the interview transcripts several times to become familiar with the data (254 single-spaced pages). Then, this same author performed open coding of the data in NVivo 1 1, and all authors discussed a sample of the data during an open-coding meeting. Among the 75 first-order codes obtained, $80 \%$ were mentioned in at least two interviews, indicating inductive thematic saturation. ${ }^{45}$ The first author grouped these first-order codes in an iterative 
process, on the basis of theory and discussions with the wider research team. This represents the only abductive step in the process, to establish "a dialogue between theory and empirical material" (p. 1270). ${ }^{47}$ One of the discussions with the research team resulted in the identification of a suitable theoretical framework (i.e. selfdetermination theory [SDT], as we present subsequently). This theoretical framework marked a shift from induction to deduction in the data analysis process; the subsequent steps used this theoretical framework to group the first-order codes into second-order categories, which were based on the theoretical framework. That is, some of the data analysis steps are inspired by abductive methodologies, but rather than being purely abductive, our process combines inductive steps with several deductive steps, with one abductive step in the middle (Figure 2). ${ }^{46}$

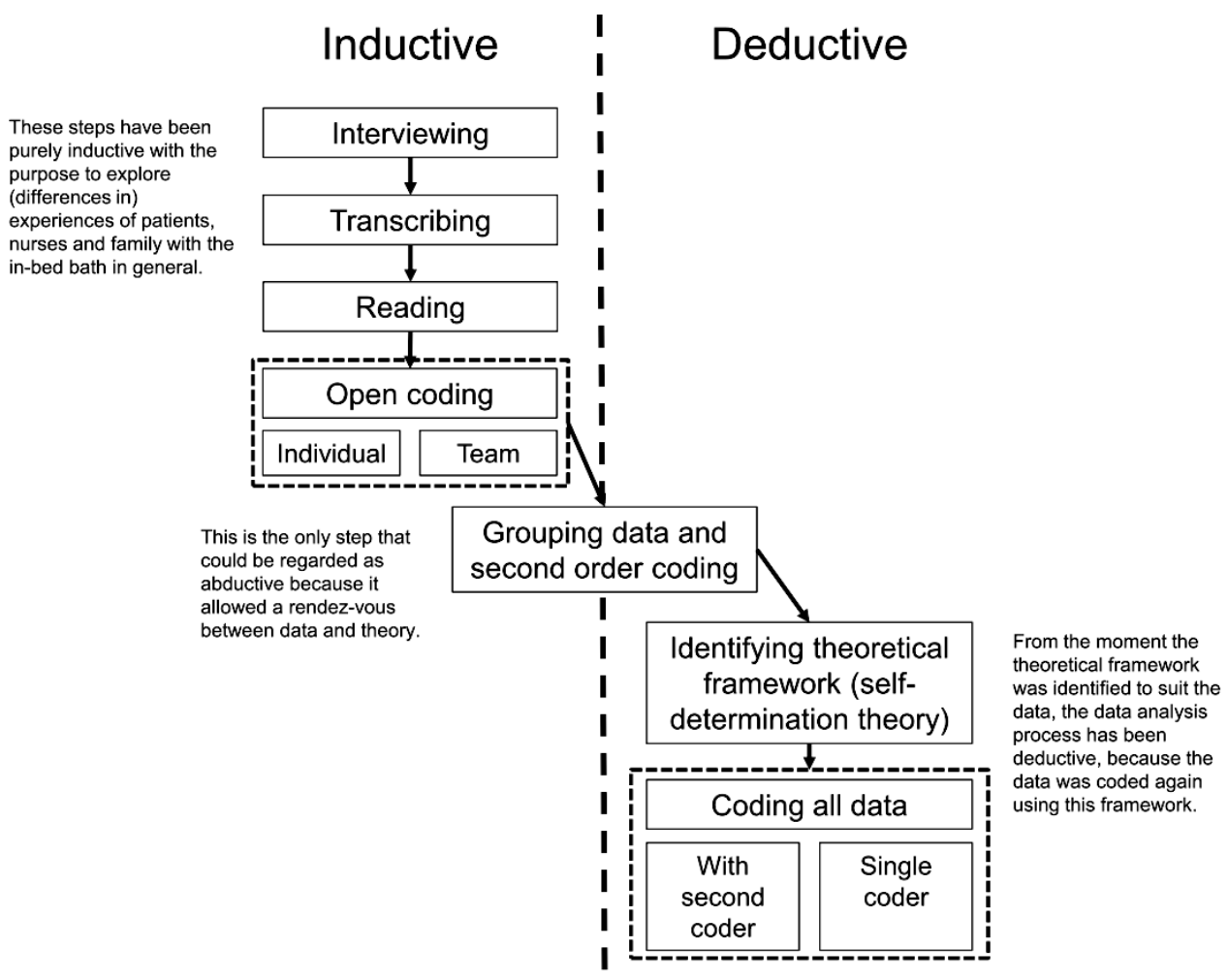

Figure 2 - Data analysis process

To facilitate coding by independent researchers using the identified secondorder categories, the first author unitized the data samples. ${ }^{48}$ After coding two data samples, the researchers discussed their findings, which aided in the interpretation of the data. Next, the first author coded the rest of the data. Although the point of true saturation cannot be determined, most first-order codes could be grouped in one of the second-order categories, which suggests a priori thematic saturation. ${ }^{45}$ Finally, the 


\section{Chapter 2}

first author integrated these data by mapping relationships among the second-order categories and discussed the findings with the whole research team. ${ }^{49}$

\section{FINDINGS}

\section{Actors' interests and needs}

The analysis of the data uncovered several actor interests and needs. So far, we have used the terms 'interests' and 'needs' interchangeably; in the presentation of the results, we simplify the wording and refer to needs, which encompass both forms.

\section{Needs of the focal actor}

First, patients (i.e. the focal actors) seek control over their bathing, which became apparent in their descriptions of experiences in which they lacked such control, such that when 'you get that nurse and then you get that nurse, I don't complain. There is not anything I can choose anyways' (Mrs. Green). Second, patients need to gain a sense of self-confidence from performing some elements of bathing by themselves, as signaled by both positive and negative reports, such as the assertions 'I enjoy that I can still wash parts of my body myself' (Mr. Taylor) or 'If I lie in bed, I often think "I wish I could bathe myself." Now I cannot do anything anymore' (Mrs. Brown). Third, patients expressed their need for normality after a bath (e.g. undertaking activities similar to those performed by healthy persons): 'For myself and for others I think it is good to be bathed. So I feel capable to be normal again' (Mr. Taylor). As a negative example, one patient noted a lack of normality: 'Actually, a person should feel fit after a bed bath. Unfortunately, I don't have that luck, probably because of the condition of my lungs' (Mr. Thomas). Fourth, patients cited their need for safety, emotionally and physically, in their direct and intimate interactions with nurses during the bed baths, such as a patient who explained, 'Most of the time, I am bathed by two nurses. Because they have to turn me around, and then they have to firmly hold me because I am terrified that I will fall out of bed' (Mrs. Green). Fifth, patients indicate a need for pleasant relationships with nurses: 'I would have preferences for certain nurses because of the way they treat me' (Mr. Thomas).

Frontline employees' (FLE) needs

The first need among nurses (i.e. FLEs) is to act according to their personal preferences (i.e. as human beings, not as nurses): 'Personally, I like to primp women' (Nurse Polly). Yet the nurses also noted the necessity to put their personal preferences aside sometimes to meet patients' or families' requests, which limited their ability to organize the bed bath encounter as they preferred. Second, nurses also expressed their need for professionalism, in describing situations that identified them as skilled professionals able to achieve nursing goals (e.g. patient satisfaction): 'But I also must take care of patients that I cannot really get along with. In those cases, I have to put my thoughts aside and simply make the best of it. And afterwards, if everything went well I think to 
myself, "Oh, well, fine, if he is satisfied then I am as well"' (Nurse Kim). On some occasions, however, nurses worry about doing their job professionally, often due to contextual factors such as staff shortages: 'You see, last weekend, one colleague could not come to work and then it is only the three of us working at this ward. In those cases, I can get annoyed because I know, "Oh, I still need to take care of that many people"' (Nurse Polly). Third, similar to patients, nurses expressed a need for safety. If patients and nurses get along well, nurses feel emotionally comfortable, but other encounters cause them emotional discomfort (e.g. patients actively asking nurses to bathe intimate body parts). Fourth, nurses also acknowledge their need for pleasant relationships, as indicated by their descriptions of their relationships with patients: 'I like that you can see that they appreciate what I'm doing and that they show interest in my personal life, even though they are patients living in the nursing home. If they would not ask personal things, I would feel less satisfied, because then you only pay attention to them and they do not care about me' (Nurse Alice).

\section{Transformative service mediators' (TSM) needs}

In contrast with patients and nurses, family members (i.e. TSMs) generally are not physically present during the bed bath. Many family members interviewed for this study indicated that they were not even aware of how their loved one is bathed. Still, two needs emerged. First, these informants indicated their need for self-assurance; they want to feel confident about their abilities as informal caregivers. Family members who believed the patients were being properly cared for in the nursing home also felt assured that they were living up to their responsibility to protect patients' well-being. Others felt guilt though, due to their concerns about insufficient care provision: "It makes me happy that I can see that they take care of her hair and her nails. That makes me happy, then I think, "Thank God." Imagine, she would neglect herself and she would say "I don't need to have my hair done." That would hurt. Then I would think, "Oh no, I put her somewhere where she does not want to be." I wouldn't like that, not for her and not for myself' (daughter Bessie). Second, this study identified family members' need for relational maintenance through ties with both patients and nurses. One family member indicated both the importance of such good relationships and the challenge of maintaining them with nurses and the patient simultaneously, because the patient was dissatisfied with her care: 'You see, I want to protect my mother but also the nurses. I feel stuck in between my mother and the nurses, which can be very difficult sometimes' (daughter Bessie).

Psychological needs as a lens to group actors' needs

During the data analysis process, the author team engaged in several rounds of discussion and realized that the actors' needs presented so far closely matched the basic psychological needs of autonomy, competence, and relatedness, which are central to SDT. SDT is a motivational theory that highlights the importance of psychological needs as determinants of optimal well-being in daily life. 50 Autonomy, competence, and relatedness are innate and universal needs and therefore are 
considered higher-order needs that explain the origins of actors' lower-order interests and needs. In other words, we regard lower-order needs as actor-specific manifestations of basic psychological needs. Autonomy implies a need to experience control and self-organize experiences and behaviors. ${ }^{29,}{ }^{51}$ In our study, autonomy is reflected in focal actors' lower-order need for control (e.g. having a say in which nurse provides the bed bath) and FLEs' lower-order need to meet their personal preferences (e.g. primping patients). Competence refers to an experience of proficiency in performing tasks in physical and social contexts, as represented by focal actors' lower-order needs for self-confidence and normality (i.e. to be proficient in their daily lives), FLEs' lower-order need for professionalism (i.e. to be proficient as a nurse), and TSMs' lower-order need for self-assurance (i.e. to be proficient as an informal caregiver). ${ }^{29}, 51$ Finally, relatedness implies a desire for safe, intimate relationships and a sense of belonging, which is reflected in focal actors' and FLEs' lower-order needs for safety and pleasant relationships and TSMs' lower-order need for relational maintenance.29, 52 Fulfillment of these needs contributes to individual actor well-being. ${ }^{53,54}$ In other words, activities and relationships that fulfill the needs of autonomy, competence, and relatedness contribute to well-being, whereas ill-being results from the lack of satisfaction of these needs..$^{50}$ In this study, ill-being represents a deprivation of actors' mental well-being (e.g. patients experiencing anxiety or low levels of vitality, nurses experiencing low job satisfaction), caused by actors' experiences with the bed bath that negatively affect the fulfillment of their psychological needs.

\section{Effect of tensions and alignments in actors' psychological needs on network well- being}

Actors try to fulfill their needs through co-creation of services. ${ }^{14}$ If tensions exist among actors' psychological needs, not all actors' lower-order needs, related to their psychological needs, are being fulfilled simultaneously in co-creation of services. By contrast, alignments imply the fulfillment of multiple actors' needs at once.

By accounting for interrelationships among actors, we move from the actor to the network level. Our study data provide rich, illustrative evidence of how cocreation of services (which actors initiate to fulfill their own psychological needs) affects the need fulfillment of other actors and thus determines balanced centricity. Because most illustrative evidence refers to interrelationships among focal actors and FLEs, we focus on these dyads in this section. However, tensions and alignments between TSMs on the one hand and focal actors and FLEs on the other hand also emerge.

Tensions and alignments: focal actors' needs and network well-being Patients' (i.e. focal actors') choice to participate in the bed bath or not, to fulfill their autonomy- or competence-based needs, can affect nurses' (i.e. FLEs') need for autonomy. Nurse Polly explained how a patient's unwillingness to bathe parts of his body himself impedes her need fulfillment: 'If I ask him if I need to wash some parts of 
his body, he simply tells me to do that. But he is able to wash those parts himself. That really bothers me.' Her personal preference that the patient washes parts of his body himself is overruled by the request of this focal actor and his unwillingness to participate. Patients' participation also enhances nurses' competence-based need for professionalism. That is, nurses' need for competence can be fulfilled when their work is facilitated by patients who assist during the bath: 'For some patients I need only 20 minutes to give a bed bath, whereas others might take an hour for example. If I have to bathe patients who can participate, I'm finished earlier and I can easily bathe eight patients a day' (Nurse Florence).

Other illustrative evidence relates to patients who share information with nurses to fulfill their own autonomy-based need for control, a step that can make nurses feel empowered to provide a proper bath, which then fulfills their need for competence, such as when patients offer insights into their preferences: "I think most patients want to look neat and cared for, but many cannot take care of that themselves, and then we need to do it. Sometimes patients share their preferences and otherwise the family does' (Nurse Alice). However, if patients' preferences deviate too much from nurses' routines and standards, it is difficult for nurses to meet them in a professional way. For example, Nurse Florence explained the difficulty of meeting patient preferences with respect to timing: 'Bathing at the request of the patient could be possible but would have major consequences. We would not have enough personnel in the afternoon to bathe people, so we then would need to reorganize our whole process' (Nurse Florence).

Apart from sharing information, patients interact with nurses to invest in their relationships and thereby fulfill their need for relatedness, which also affects nurses' need fulfillment. Pleasant interactions with patients can ease care provision and fulfill nurses' need for competence: 'It is more pleasant to do my job and also for the person who I am taking care of. It is more convenient if they like to see me. It seems to be easier as well' (Nurse Polly). If instead patients display negative moods in their interactions, it becomes more difficult for nurses to do their jobs professionally: 'If the patient is in a bad mood, the care provision process runs less pleasantly because if he is angry, he can reject care by asking us to leave him alone. That is tough because it then takes longer to take care of him' (Nurse Florence).

Finally, patient interactions influence nurses' need for relatedness. Nurse Polly explains the positive outcomes when patients show appreciation for her efforts: 'If patients appreciate what you are doing for them, that they are taken care of, you also get a better relationship with them.'

Tensions and alignments: frontline employees' (FLE) needs and network well-being Several pieces of illustrative evidence indicate positive or negative impacts of nurses' (i.e. FLEs') adherence to nursing routines and policies, to fulfill their own competencebased need for professionalism, on patients' (i.e. focal actors') needs. For example, nurses provide patient-centered care, which requires accounting for the patients' preferences and accepting their directions, which in turn makes the nurses feel 
professional. Such efforts can have a positive impact on patients' need for autonomy, as noted by one patient: 'I frequently had wounds on my groin. Since they bathe me with clean water and Sunlight soap, it healed. I insisted on the use of that' (Mrs. Smith). Nursing policies also often prescribe the encouragement of patient participation in the care process. Such encouragement can have a positive impact on patients' competence-based need for self-confidence. For example, Nurse Kim described encouraging a patient to wash himself at least partially: 'No, generally, he helps where he can during the bed bath. You just give him a washing mitt and then you pay attention to what he can do himself and I take over the rest.' However, nurses sometimes follow nursing routines and policies that make them feel competent but impede the fulfillment of patients' autonomy-based need for control: 'We live up to patients' requests unless this is not possible due to agreements with doctors or the family' (Nurse Alice). Here, Nurse Alice allows agreements made with doctors or the family to prevail over patient preferences to satisfy her interest for professionalism. The time at which nurses give bed baths also often depends on nursing routines, rather than patients' preferences, which could affect patients' ability to engage in other activities: 'I cannot always go to the physiotherapist. Because sometimes they bathe me too late, and I cannot make it' (Mrs. Smith). Mrs. Smith cannot undertake other activities, as any 'normal' person can, which impedes the fulfillment of her competence-based need for normality.

In addition to following nursing routines and policies, nurses seek information, mainly to fulfill their own competence-based need for professionalism, which influences the fulfillment of patients' autonomy-based need for control, particularly if nurses actively seek information about patients' preferences: 'Yes, we also bathe some patients with water and soap. We ask our patients if they want to be bathed with washing without water or with water and soap' (Nurse Florence).

Finally, nurses generally interact with patients to satisfy their own needs for relatedness or competence, which can also improve patients' need fulfillment. With regard to patients' relatedness-based need for pleasant relationships, we identify a positive effect in a quote from one of the nurses, explaining efforts to enter into oneon-one interactions with patients to provide personal attention, as part of professional care: 'Usually, I bathe patients all by myself. Most patients don't feel comfortable being bathed by two nurses because then the nurses have a talk with each other and don't involve the patient in the conversation' (Nurse James). This illustrative evidence also implies a potential negative effect of a lack of personal attention; the patient might miss a sense of having a pleasant relationship if bathed by two nurses and excluded from their interactions. One patient confirmed the importance of personal contact in terms of a need for relatedness: 'Yes, it is nice to get attention from the nurses, then you feel at home' (Mrs. Smith). 
Tensions and alignments: transformative service mediators' (TSM) needs and network well-being

Available data on family members' (i.e. TSMs') interdependencies with patients (i.e. focal actors) and nurses (i.e. FLEs) illustrate some notable actor experiences. For example, in the patient-family dyad, illustrative evidence shows a positive effect of family members' activities (to fulfill their own competence-based need for selfassurance), such as organizing patients' clothes, on patients' autonomy-based need for control: "I tell the nurses "In the closet you can find my clothes." My daughter makes sure the right clothes are prepared. My daughter says, "Mother, I hung up your clothes here so they can put you in these"' (Mrs. Brown). Conversely, family members' personal interactions with the patient (which might be executed to fulfill their own relatedness-based need for relational maintenance) can negatively affect patients' autonomy-based need for control. One family member persuaded a patient, her mother, to accept being bathed by a male nurse: "My mother had struggles being bathed by a male nurse in the beginning. I told my mother, "Listen mother, it is normal to be bathed by a man." Now she takes it for granted' (daughter Bessie). In this case, the patient's preference for female nurses was ignored by her daughter, who persuaded the patient to accept male nurses. The daughter did so to avoid unpleasant bed bath encounters for male nurses, in that the patient otherwise would have resisted the bath.

In the opposite direction, the fulfillment of a family member's competencebased need for self-assurance can be influenced by the information shared by patients, often in the form of complaints. Patients complain to fulfill their own autonomy-based need for control, in an effort to trigger other actors to undertake action to improve the patients' care experiences. At the same time, patients' complaints can make family members feel less competent as informal caregivers: 'I would find it terrible if my mother would tell me that she did not get a shower for over two weeks' (daughter Bessie).

Interdependencies also arise between family members and nurses. For example, family members who share information with nurses (to fulfill their competence-based need for self-assurance) can affect nurses' competence-based need for professionalism, which tends to be positive if the information helps the nurse provide better care: 'Sometimes, the family tells us that the patient smells. Family can detect that easier than us because we are constantly working at the ward and therefore don't always notice smells' (Nurse James). This information helps nurses provide services to help the patient smell nice again. Conversely, family members sometimes share information through complaints, which might negatively affect nurses' need for competence: 'Personally, I think it is very important that family members are satisfied as well [with the bed bath]. Otherwise, they will complain. That would make me think I am not doing my job well' (Nurse Kim).

Nurses' activities in co-creation of services in turn can influence family members' competence-based need for self-assurance, as illustrated in the following quote: 'I heard many times that patients sometimes don't want to be bathed but that 
the family demands us to give a bed bath. "Just give her something that will relax her like a tranquilizer so you can bathe her." We are helpless in such cases. That is not allowed' (Nurse Kim). Nurses observe nursing policies to fulfill their own competencebased need for professionalism, so they cannot tranquilize patients to bathe them. Subsequently, the patient is not bathed, which may leave the family feeling less competent because they failed to assure what the family perceives to be proper care. As a positive effect, nurses who actively ask family members for information (to fulfill their own competence-based need for professionalism) offer these actors a means to protect patients' well-being and fulfill their need for competence: 'We organize meetings with family to discuss problems and come up with solutions together' (Nurse Fred).

\section{DISCUSSION}

\section{Theoretical contributions}

Health care services can enhance the well-being of vulnerable patient populations and other health care actors. ${ }^{2}$ However, the involvement of many actors, each with their own interests, makes health care highly complex, which influences actor and network well-being. ${ }^{38}$ Therefore, this study explores actors' experiences from a novel multi-actor perspective. Drawing on multiple actors' experiences, this study proposes balanced centricity as an indicator of network well-being. ${ }^{12}$ To assess balanced centricity, we focus on the multi-actor service setting of bed baths-a recurring, cocreated service encounter related to basic care-which was associated with an imbalance identified in prior research. ${ }^{6}$ The results we derived from our in-depth interviews with focal actors (i.e. patients), FLEs (i.e. nurses), and TSMs (i.e. family members) suggest the conceptual model in Figure 3, which depicts the influence of tensions and alignments in psychological needs across actors on balanced centricity through co-creation of services. The conceptual model addresses our research questions.

The first and second research questions center on actors' interests and needs in co-created service encounters. In response to the first research question, our empirical, multi-actor research approach reveals that the interests and needs of all three actors differ. Focal actors need control, self-confidence, normality, safety, and pleasant relationships; FLEs express needs for personal preferences, professionalism, safety, and pleasant relationships; and TSMs mainly indicate needs for self-assurance and relational maintenance. Although actors' interests and needs differ, SDT emerges as instrumental for classifying these interests and needs according to higher-order psychological needs of autonomy, competence, and relatedness. ${ }^{51}$ This finding addresses our second research question, which pertains to the origin of tensions and alignments between interests and needs of different actors. That is, universal, often unconscious, psychological needs are higher-order needs that give rise to actorspecific, more conscious, lower-order needs. 


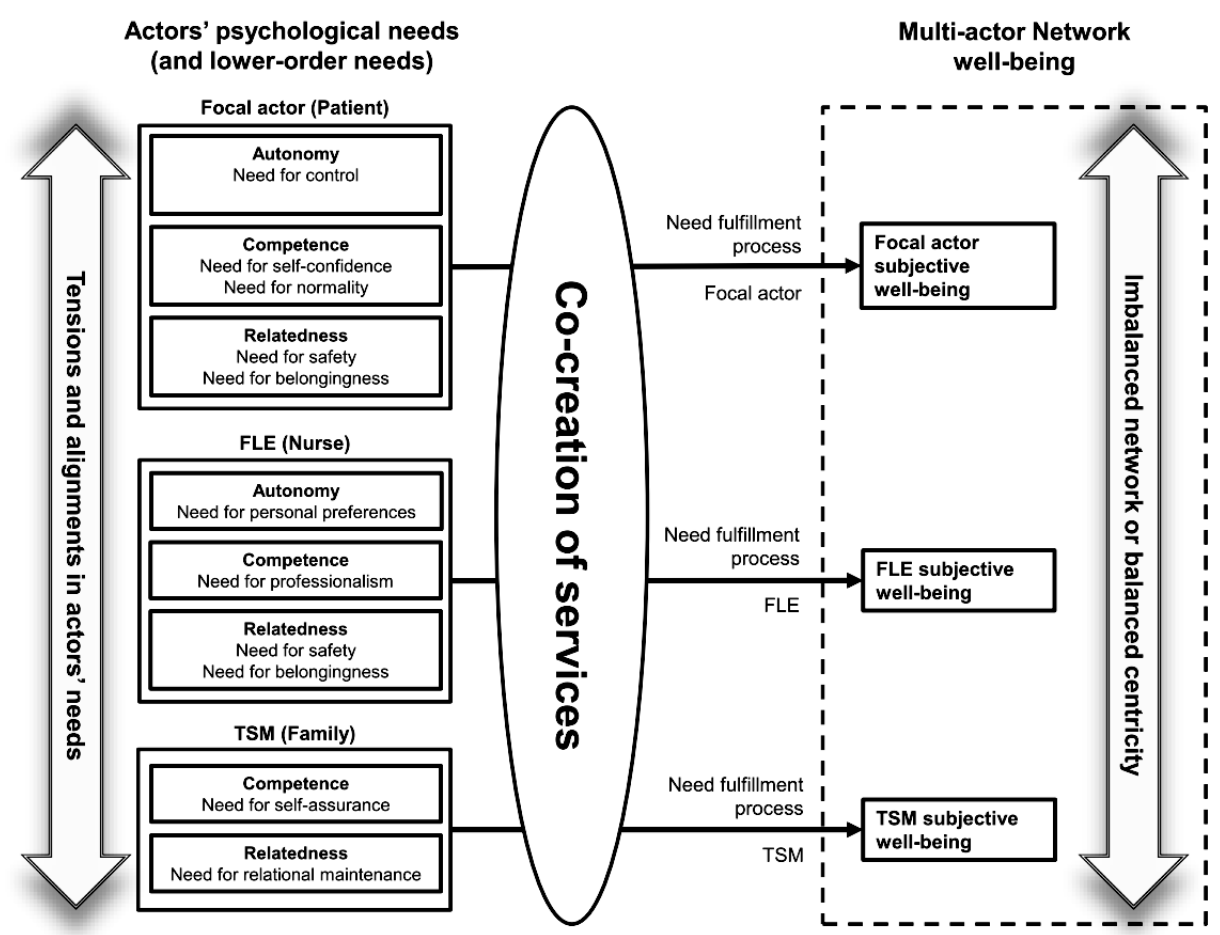

Figure 3 - Conceptual model

As a motivational theory, SDT suggests that the basic psychological needs of autonomy, competence, and relatedness explain actors' goal pursuit and that people are intrinsically motivated to perform activities that they believe will contribute to these innate psychological needs. ${ }^{51,53}$ In our conceptual model, actors express different interests and needs (e.g. patients' need for self-confidence) that stem from their psychological needs (e.g. competence), which they try to satisfy through cocreation of services (e.g. patients execute parts of the bed bath themselves). When actors can fulfill their interests and needs by co-creating services, it satisfies their higher-order psychological needs, resulting in their increased well-being. Prior studies similarly predict the achievement of actor well-being through the realization of basic and higher needs, which the current study extends by exploring multiple actors' needs simultaneously. ${ }^{17}$ Furthermore, we answer Osei-Frimpong's call for research that takes an SDT perspective to assess multiple actors' motivations to co-create and determine how consequent actor behaviors affect other actors. ${ }^{35}$

Our third research question pertains to how tensions and alignments between interests and needs across actors affect actors' well-being and the achievement of balanced centricity, as an indicator of multi-actor network well-being. Our results show that actors aim to fulfill their needs through co-creation of services, which affects the fulfillment of other actors' needs as well, whether positively or negatively, with 
relevant implications for these other actors' well-being, as well as network well-being. In the conceptual model in Figure 3, balanced centricity is enhanced if all actors' psychological needs are satisfied, such as when the lower-order interests and needs of all actors are aligned, enabling all actors' interests and needs to be fulfilled simultaneously through co-creation of services. If tensions arise between the interests and needs of different actors though, not all actors' psychological needs can be satisfied, which impedes balanced centricity and ultimately network well-being. The vertical two-pointed arrow on the left in our conceptual model reflects these tensions and alignments between actors' needs; the similar arrow on the right depicts the effect of these tensions and alignments on network well-being.

To increase network well-being, the psychological need fulfillment of all actors is important, not only for those directly involved in co-creation of services (i.e. patients and nurses) but also for other actors, such as TSMs, who support vulnerable focal actors in the co-creation process (i.e. family members).' If tensions between TSMs and other actors' interests and needs exist, network well-being could be threatened by TSMs' activities in co-creation of services that impede the fulfillment of other actors' psychological needs. For example, family members' complaints (which they raise to ensure proper care provision to the patient and to satisfy their own competence-based need for self-assurance) can negatively affect nurses' need for competence (i.e. nurses feel they did not do a good job).

In particular, psychological need fulfillment should increase network wellbeing in a transformative setting such as health care. However, because well-being is generally described as multidimensional, optimal well-being can be realized only if all possible actors' needs are fulfilled. ${ }^{17}$ Yet, as the current study demonstrates, psychological needs are vital for network well-being in a TSR setting, featuring vulnerable focal actors who depend on other actors, such as patients depending on nurses and family. In this sense, our study contributes to TSR and its efforts to increase vulnerable focal actors' well-being. ' Furthermore, we contribute to literature on wellbeing by exploring both individual actor well-being and well-being at a network level. ${ }^{7}$

In response to the need for multi-actor perspectives, this study of multi-actor networks also extends the research on balanced centricity by empirically establishing crucial roles of both tensions and alignments across the needs of different actors. ${ }^{16,21}$, 55 By showing that balanced centricity is an indicator of network well-being, this study advances discussions of network well-being to a more concrete, actionable level. The empirical results refer to bed baths, which represent a specific, crucial, and recurring co-created service encounter in health care services. We predict that the findings are relevant to other transformative service encounters as well, especially in health care, such as feeding patients or medical checkups.

\section{Managerial implications}

$\mathrm{Ng}$ et al. identify different styles that service providers can adopt to support cocreation; 55 this study specifies that health care providers should particularly support 
actors' psychological need fulfillment to facilitate their well-being (e.g. offering patients ways to fulfill their need for competence by letting them wash parts of their bodies themselves, giving family members opportunities to protect patient well-being to fulfill their need for competence). FLEs should be aware of the consequences of their own activities on other actors' needs to prevent negative effects and identify other opportunities to contribute to actor and network well-being. ${ }^{36}$ This recommendation is especially pertinent to health care, in which focal actor wellbeing depends heavily on how the care is provided, because focal actors lack the knowledge to assess its technical quality. ${ }^{56}$ That is, the interaction between nurses and patients is critical for patient well-being. ${ }^{57}$

Balanced centricity arguably might not be realistically feasible in a highly complex service system featuring vulnerable actors. Based on a multi-actor approach, our findings suggest two managerial recommendations that could contribute to balanced centricity and that are generalizable to other health care encounters as well: (1) grant control to vulnerable focal actors by assessing their needs and (2) stimulate all network actors to participate in co-creation of services.

\section{Grant control to vulnerable focal actors (patients)}

A key goal should be to identify and eliminate tensions among actors' needs, especially between vulnerable focal actors' (e.g. patients') need for autonomy and other actors' needs. Especially FLEs should be aware of their important role, because they can provide vulnerable focal actors a sense of control by supporting them in the co-creation of services. ${ }^{3}$ The data emphasize the importance of autonomy in relation to focal actors' well-being but also suggest the limits of such autonomy. Notably, focal actors' autonomy is constrained by their lack of choice, as might be caused when FLEs stick with routines to fulfill their own need for competence. For example, despite evidence that washing without water is at least as effective as bed baths with water and soap, several nurses expressed doubts about the effectiveness of washing without water and therefore continued to bathe patients with water and soap.58 These nurses also might have remained unaware of patients' preferences; many patients indicated they would rather receive baths provided without water.

Service managers can resolve such tensions between vulnerable focal actors' autonomy-based needs and FLEs' competence-based needs by encouraging frequent, active information exchanges. With respect to the bed bath, nurses might change their mind about washing without water if patients (actively or reactively) shared their preferences every time they were bathed. Several pieces of illustrative evidence also indicate that nurses feel more competent when patients share their preferences, which helps them deliver proper care. At the same time, patients gain control over the bed bath when they can share their preferences. Consequently, patients' and nurses' control balance better, which improves both actors' well-being. ${ }^{24}$ For other health care encounters, such as feeding patients or subscribing medicine, health care workers similarly might deviate from routines they personally find effective if they are aware of actors' preferences. If FLEs have a means 


\section{Chapter 2}

to accommodate focal actors' preferences without jeopardizing their own need fulfillment (e.g. bathing body parts in a certain order), care provision will be more effective for both parties involved.

Balanced centricity thus could be enhanced if FLEs frequently collect information on focal actors' needs and deliver service accordingly, especially in tasks that they execute routinely but that also have a significant impact on the well-being of vulnerable focal actors. Service managers could facilitate information exchanges by training FLEs and by introducing supportive tools. For example, nurses could wear buttons that encourage patients to 'Tell me what you need today' and thereby invite patients to share their preferences. Finally, when vulnerable focal actors cannot share preferences (e.g. cognitively impaired patients), other actors (e.g. TSMs, such as family) should be involved more, which could improve the focal actors' autonomy and well-being. ${ }^{1}$

Stimulate all network actors to participate in co-creation of services

In health care, family involvement has the potential to contribute to network wellbeing, as suggested by previous research that defines health care as a team effort, involving patients, professional caregivers, and families. ${ }^{26,59}$ Increased family (i.e. TSMs) involvement could contribute to all actors' needs. For FLEs, TSMs represent important information sources to learn about focal actors' preferences, so involving TSMs could fulfill FLEs' need for competence. At the same time, TSMs would have more opportunities to protect focal actor well-being and thereby fulfill their need for competence. Finally, this approach would enhance focal actors' autonomy, because the TSM can match focal actors' preferences. Unfortunately though, in our study, family members expressed little knowledge about bed bathing processes.

This lack of knowledge might indicate a lack of communication among actors, which also can lead to imbalances. ${ }^{6}$ Health care managers should find ways to build co-creation cultures that "support all actors in actively participating in resource integration" (p. 439).60 For example, health care managers could organize 'nurse-for-a-day' meetings, in which family members join the nurse in performing care provision activities with the patient. During these interactions, all actors gain opportunities to discuss care preferences and identify potential tensions, such as when family and nurses have opposing views on what is best for the patient. ${ }^{59}$

Finally, this study encourages health care managers to foster longitudinal (vs. episodic) relationships primarily between patients and nurses, but also between family members and nurses. ${ }^{7}$ Longitudinal relationships could be realized by assigning the same nurses to the same patients (and their families). Such an approach could help patients, nurses, and family members gain better knowledge about each other's needs, which could be taken into account during key recurring service encounters such as bed baths. As a result, individual actor well-being and network well-being would increase. For example, patients' need for autonomy could be fulfilled if their preferences are met more often because of nurses' increased knowledge about these preferences; nurses' need for competence could be fulfilled if they know that 
they contributed to patient satisfaction with the bed bath; and family members' need for competence could be fulfilled if they have a central nurse to talk to in order to protect patients' well-being.

\section{Limitations and further research}

This exploratory study offers a foundation for continued research that expands understanding of the interrelationships between actor and network well-being. The findings are particularly valuable for transformative systems in which well-being is critical, such as health care. We focused on network actors that we deemed most relevant to bed bath service provision, namely, patients, nurses, and families. Studies also could expand to include larger networks of other actors, such as doctors, friends, and support groups. Furthermore, the chosen context offers valuable insights into actors' needs and well-being, and applying the model to other (health care) services could be similarly insightful. Family members' involvement in bed bathing appears relatively minimal, and patients generally are rather passive. Accordingly, it would be useful to apply this study's model to service settings in which actors are more involved and more willing to initiate the co-creation of services. Other applications could refer specifically to the impact of tensions across health care encounters (e.g. how tensions during the bed bath affect subsequent health care encounters, such as receiving meals) and explore how actors cope with these tensions over time.

Finally, further research could address the influence of contextual factors on actors' needs. We note several contextual factors that appear to affect actors' need fulfillment, including health care structures and institutions (e.g. morning bathing routines), resource availability (e.g. sufficient nurses), and technology adoption (e.g. washing without water). New technologies encourage the development of innovative services that can create beneficial outcomes for multiple actors. ${ }^{5}$ In line with our argument that fulfilling all actors' psychological needs is critical to network well-being, we call for research that clarifies the influence of technological components within service systems on actors' psychological need fulfillment. In general, exploring the impact of contextual factors on actors' need fulfillment could facilitate the achievement of more balanced service systems. 


\section{References}

1. Johns R and Davey J. Introducing the transformative service mediator: value creation with vulnerable consumers. Journal of Services Marketing 2019:33:5-15.

2. Ostrom AL, Parasuraman A, Bowen DE, et al. Service research priorities in a rapidly changing context. Journal of Service Research 2015;18:127-159.

3. Virlée J, van Riel ACR and Hammedi W. Health literacy and its effects on well-being: how vulnerable healthcare service users integrate online resources. Journal of Services Marketing 2020;34:697-715.

4. Leroi-Werelds S. An update on customer value: state of the art, revised typology, and research agenda. Journal of Service Management 2019;30:650-680.

5. Patrício L, Gustafsson A and Fisk R. Upframing service design and innovation for research impact. Journal of Service Research 2018;21:3-16.

6. Verleye K, Jaakkola E, Hodgkinson IR, et al. What causes imbalance in complex service networks? Evidence from a public health service. Journal of Service Management 2017;28:34-56.

7. Berry LL, Attai DJ, Scammon DL, et al. When the aims and the ends of health care misalign. Journal of Service Research 2020:1-25.

8. Suárez-Álvarez L, Suárez-Vázquez A and del Río-Lanza A. Companion cocreation: improving health service encounters of the elderly. Journal of Services Marketing 2020;35:1 16-130.

9. Taiminen $H$, Taiminen $K$ and Munnukka J. Enabling transformative value creation through online weight loss services. Journal of Services Marketing 2020;34:797-808.

10. Anderson S, Nasr L and Rayburn SW. Transformative service research and service design: synergistic effects in healthcare. Service Industries Journal 2018;38:99-113.

11. Leo WWC, Laud G and Chou CY. Service system well-being: conceptualising a holistic concept. Journal of Service Management 2019;30:766-792.

12. Gummesson E. Exit services marketing - enter service marketing. Journal of Customer Behaviour 2007:6:113-141.

13. Quero MJ and Ventura R. The role of balanced centricity in the Spanish creative industries adopting a crowd-funding organisational model. Journal of Service Theory and Practice 2015;25:122-139.

14. Oertzen A, Odekerken-Schröder G, Brax SA, et al. Co-creating services - conceptual clarification, forms and outcomes. Journal of Service Management 2018;29:641-679.

15. Grönroos C and Voima P. Critical service logic: making sense of value creation and cocreation. Journal of the Academy of Marketing Science 2013;41:133-150.

16. McColl-Kennedy JR, Vargo SL, Dagger TS, et al. Health care customer value cocreation practice styles. Journal of Service Research 2012;15:370-389.

17. Falter $M$ and Hadwich K. Customer service well-being: scale development and validation. Service Industries Journal 2020;40:181-202.

18. Vargo SL and Lusch RF. Evolving to a new dominant logic for marketing. Journal of Marketing 2004;68:1-17.

19. Dean A and Indrianti N. Transformative service research at the BoP: the case of Etawa goat farmers in Indonesia. Journal of Services Marketing 2020;34:665-681.

20. Vargo SL and Lusch RF. The SAGE handbook of service-dominant logic. SAGE Publications Inc. 2018. 
21. Sweeney JC, Danaher TS and MCColl-Kennedy JR. Customer effort in value cocreation: improving quality of life and behavioral intentions of health care customers. Journal of Service Research 2015;18:318-335.

22. Hillebrand $\mathrm{B}$, Driessen $\mathrm{PH}$ and Koll $\mathrm{O}$. Stakeholder marketing: theoretical foundations and required capablities. Journal of the Academy of Marketing Science 2015;43:41 1428.

23. Plé $L$ and Cáceres RC. Not always co-creation: introducing interactional co-destruction of value in service-dominant logic. Journal of Services Marketing 2010;24:430-437.

24. Black HG and Gallan AS. Transformative service networks: cocreated value as wellbeing. Service Industries Journal 2015;35:826-845.

25. Davey J and Grönroos C. Health service literacy: complementary actor roles for transformative value co-creation. Journal of Services Marketing 2019:33:687-701.

26. McColl-Kennedy J, Hogan SJ, Witell L, et al. Cocreative customer practices: effects of health care customer value cocreation practices on well-being. Journal of Business Research 2017;70:55-66.

27. Čaić M, Odekerken-Schröder G and Mahr D. Service robots: value co-creation and codestruction in elderly care networks. Journal of Service Management 2018;29:178-205.

28. Vargo SL, Akaka MA and Vaughan CM. Conceptualizing value: a service-ecosystem view. Journal of Creating Value 2017;3:117-124.

29. Sharma S, Conduit J and Hill SR. Hedonic and eudaimonic well-being outcomes from co-creation roles: a study of vulnerable customers. Journal of Services Marketing 2017:31:397-411.

30. Frow P, McColl-Kennedy JR and Payne A. Co-creation practices: their role in shaping a health care ecosystem. Industrial Marketing Management 2016:56:24-39.

31. Gummesson E. Relationship marketing and a new economy: it's time for deprogramming. Journal of Services Marketing 2002;1 6:585-589.

32. Gummesson E. Extending the service-dominant logic: from customer centricity to balanced centricity. Journal of the Academy of Marketing Science 2008:36:15-17.

33. Reypens $C$, Lievens $A$ and Blazevic $\vee$. Leveraging value in mutli-stakeholder innovation networks: a process framework for value co-creation and capture. Industrial Marketing Management 2016:56:40-50.

34. Neghina C, Bloemer J, Van Birgelen $M$, et al. Consumer motives and willingness to cocreate in professional and generic services. Journal of Service Management 2017:28:157-181.

35. Osei-Frimpong K. Patient participatory behaviours in healthcare service delivery. Journal of Service Theory and Practice 2017;27:453-474.

36. Findsrud R, Tronvoll B and Edvardsson B. Motivation: the missing driver for theorizing about resource integration. Marketing Theory 2018;18:493-519.

37. Rosenbaum MS. Transformative service research: research that matters. Service Industries Journal 2015;35:801-805.

38. Pinho N, Beirão G, Patrício L, et al. Understanding value co-creation in complex services with many actors. Journal of Service Management 2014;25:470-493.

39. Downey L and Lloyd H. Bed bathing patients in hospital. Nursing Standard 2008;22:3540.

40. Ahluwalia SC, Gill TM, Baker DI, et al. Perspectives of older persons on bathing and bathing disability: a qualitative study. Journal of the American Geriatrics Society 2010;58:450-456. 
41. Kitson A, Conroy T, Kuluski K, et al. Reclaiming and redefining the Fundamentals of Care: Nursing's response to meeting patients' basic human needs. Adelaide, South Australia: School of Nursing, The University of Adelaide 2013.

42. Gummesson E. Qualitative research in marketing: Road-map for a wilderness of complexityand unpredictability. European Journal of Marketing 2005;39:309-327.

43. Rynes S and Gephart RP. From the editors: qualitative research and the "Academy of Management Journal". Academy of Mangement Journal 2004;47:454-462.

44. Pandit NR. The creation of theory: a recent application of the grounded theory method. Qualitative Report 1996;2:1-15.

45. Saunders B, Sim J, Kingstone T, et al. Saturation in qualitative research: exploring its conceptualization and operationalization. Quality \& Quantity 2018:52:1893-1907.

46. Gioia DA, Corley KG and Hamilton AL. Seeking qualitative rigor in inductive research: notes on the Gioia methodology. Organizational Research Methods 2012;16:15-31.

47. Alvesson $M$ and Kärreman D. Constructing mystery: empirical matters in theory development. Academy of Management Review 2007;32:1265-1281.

48. Campbell JL, Quincy C, Osserman J, et al. Coding in-depth semistructured interviews: problems of unitization and intercoder reliability and agreement. Sociological Methods \& Research 2013;42:294-320.

49. Spiggle S. Analysis and interpretation of qualitative data in consumer research. Journal of Consumer Research 1994:21:491-503.

50. Ryan RM and Deci EL. Self-determination theory and the facilitation of intrinsic motivation, social development, and well-being. American Psychologist 2000;55:68-78.

51. Deci EL and Ryan RM. The "what" and "why" of goal pursuits: human needs and the self-determination of behavior. Psychological Inquiry 2000;1 1:227-268.

52. Baumeister RF and Leary MR. The need to belong: desire for interpersonal attachments as a fundamental human motivation. Psychological Bulletin 1995;1 17:497-529.

53. Engström $J$ and Elg M. A self-determination theory perspective on customer participation in service development. Journal of Services Marketing 2015;29:51 1-521.

54. Kasser VG and Ryan RM. The relation of psychological needs for autonomy and relatedness to vitality, well-being, and mortality in a nursing home. Journal of Applied Social Psychology 1999;29:935-954.

55. Ng SC, Plewa C and Sweeney JC. Professional Service Providers' Resource Integration Styles (PRO-RIS): facilitating customer experiences. Journal of Service Research 2016;19: 380-395.

56. Horodnic AV, Apetrei A, Luca FA, et al. Rating healthcare services: consumer satisfaction vs. health system performance. Service Industries Journal 2018:38:974-994.

57. Anderson ST and Smith JS. Understanding hospital quality: the case of cumulative and balanced quality. Service Industries Journal 2018:38:182-200.

58. Groven FMV, Zwakhalen SMG, Odekerken-Schröder G, et al. How does washing without water perform compared to the traditional bed bath: a systematic review. BMC Geriatrics 2017;17:1-16.

59. Midtbust MH, Alnes RE, Gjengedal E, et al. A painful experience of limited understanding: healthcare professionals' experiences with palliative care of people with severe dementia in Nonwegian nursing homes. BMC Palliative Care 2018;17:1-9.

60. Sharma $S$ and Conduit J. Cocreation culture in health care organizations. Journal of Service Research 2016;19:438-457. 


\section{Appendix 1 - Script Interview with Patients (Nurses/Family Members)}

1. Can you tell me something about your day so far?

2. You told me, you were (the patient was/your family member was) bathed today. Is it important to be bathed and why?

3. Can you describe the bed bath for me?*

4. What are the consequences of the bed bath for (the patient/your family member) you?*

5. Are you satisfied with the bed bath (of the patient/your family member)?

6. What is the most important about the bed bath (of the patient/your family member)?*

*Laddering/probing was used to ask, for example:

- Is that (certain bed bath activity) important for you?

- Why is it important for you?

- Who was involved? What did this person do?

- Would it have been different if this person was not involved? 



\section{chapter}

\section{3}

\section{HOW DOES WASHIN G WITHOUT}

WATER PERFORM COMPARED

TO THE TRADITIONAL BED BATH FOR BEDRIDDEN PATIENTS: A SYSTEMATIC REVIEW

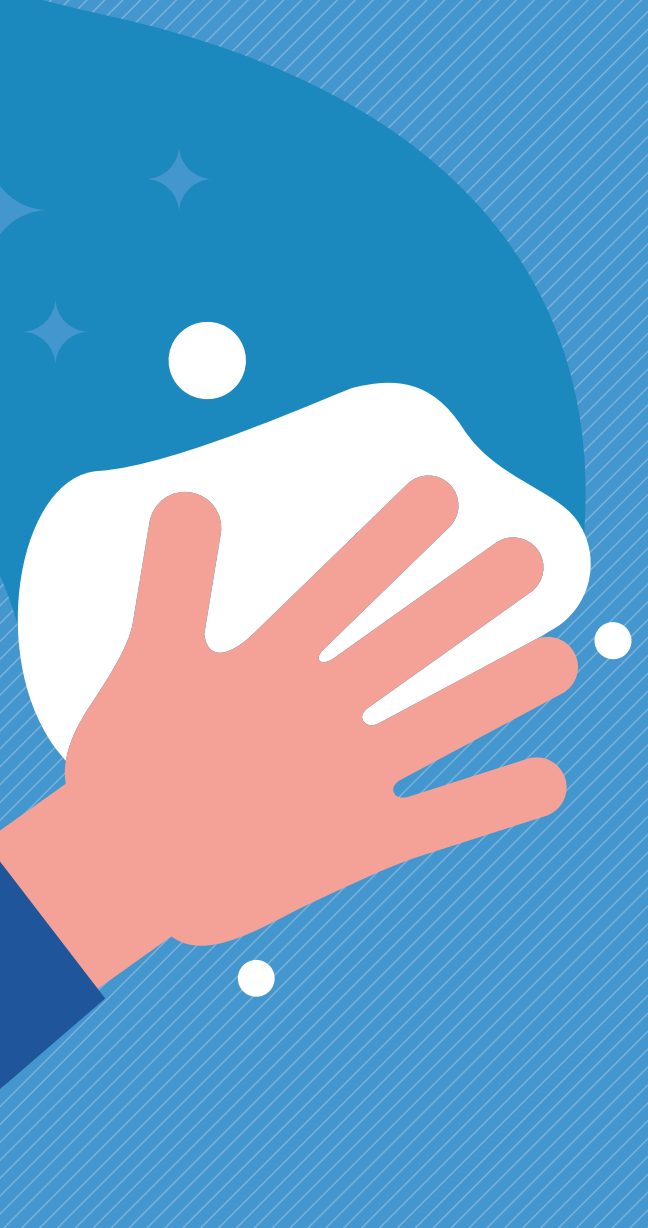

This chapter was published as:

Groven FMV. Zwakhalen SM. Odekerken-Schröder G, Joosten, EJ. and Hamers JP. How does washing without water perform compared to the traditional bed bath for be dridden patients: a systematic review. BMIC Geriatrics 2017:17:1-16 doi.org/10, 1 186/512877-017-0425-4 


\section{Chapter 3}

\section{ABSTRACT}

Background: For immobile patients, a body wash in bed is sometimes the only bathing option. Traditionally, the bed bath is performed with water and soap. However, alternatives are increasingly used in health care. Washing without water is one such alternative that has been claimed to offer several advantages, such as improved hygiene and skin condition. This systematic review aims to provide a comprehensive overview of the evidence on outcomes of the washing without water concept compared to the traditional bed bath.

Methods: Controlled trials about washing without water outcomes published after 1994 were collected by means of a systematic literature search in CINAHL, Embase, MEDLINE, and PUBMED at the $25^{\text {th }}$ of February, 2016. Additionally, references and citations were searched and experts contacted. Studies were eligible if (1) the study designs included outcomes of washing without water products developed for the full body wash compared to the traditional bed bath, and (2) they were controlled trials. Two researchers independently used a standardized quality checklist to assess the methodological quality of the eligible studies. Finally, outcomes were categorized in (1) physiological outcomes related to hygiene and skin condition, (2) stakeholderrelated outcomes, and (3) organizational outcomes in the data synthesis.

Results: Out of 33 potentially relevant articles subjected to full text screening, six studies met the eligibility criteria. Only two studies (of the same research group) were considered of high quality. The results of these high quality studies show that washing without water performed better than the traditional bed bath regarding skin abnormalities and bathing completeness. No differences between washing without water and the traditional bed bath were found for outcomes related to significant skin lesions, resistance during bathing and costs in the studies of high quality.

Conclusions: There is limited moderate to high quality evidence that washing without water is not inferior to the traditional bed bath. Future research on washing without water is needed and should pay special attention to costs, hygiene, and to stakeholder-related outcomes, such as experiences and value perceptions of patients, nursing staff and family.

\section{Acknowledgements}

The authors would like to thank Mariska Machiels for her support in the title and abstract screening and Hanneke Beerens for her support in the quality assessment of the articles. 


\section{BACKGROUND}

A nurse enters Mr. Johnson's room to give him a bed bath. Mr. Johnson is surprised that she only brings a small package with her instead of the washbasin with water, soap and towels that he would expect. After she explains to Mr. Johnson what she is going to do and after she uncovers him, the nurse opens the package and takes a washcloth. Mr. Johnson feels that the washcloth is wet when it is wiped over his face. After the first washcloth, seven other washcloths are used to bathe all his body parts separately while other body parts are covered again to mitigate cold and vulnerability. His skin dries within a minute, even though no towel is used. When the nurse is finished, she throws away all the washcloth, together with the empty package.

This hypothetical scenario represents the actual bathing practice in an increasing number of European and American health care institutions for immobile patients who, irrespective of the cause or level of immobility, need to be bathed in bed.' The package with washcloths in the example is often called "washing without water", which can be described as disposable, prepacked products for the full body wash consisting of a nonwoven carrier (washcloth) and a no-rinse cleansing fluid that allows nursing staff to bathe someone without the use of water. ${ }^{2}$ This definition of washing without water does not include products for disinfection of the body or products used to sanitize certain body areas, such as the perineum.

Personal hygiene assistance, including bathing, is a key nursing activity that is reasoned to be related to quality of life and quality of care. ${ }^{3}$ Bathing has been argued to have an effect on patient well-being and patient comfort.4, 5 Furthermore, preserving skin integrity and personal hygiene are proposed to be important physiological functions of bathing, which in turn prevent infections and disease. 6,7

The advantages of bathing in general also apply to the full body wash in bed (the bed bath), which is sometimes the only option to maintain hygiene for immobile patients who cannot bathe themselves due to chronic or acute illness. However, the most traditional bed bath with water, soap, towels and wash basins is argued to have some adverse outcomes. First of all, regarding stakeholder experiences, patients often feel dependent and uncomfortable during the traditional bed bath. ${ }^{8}$ Therefore, the bed bath can trigger aggressive and agitated behaviors, especially if patients are cognitively impaired. 3, 9, 10 Consequently, the traditional bed bath can be burdensome for both patients and nursing staff. 9,10 Furthermore, the physiological effectiveness of the traditional bed bath in terms of skin integrity and personal hygiene is questioned. For instance, Voegeli found that both soap and towel drying disrupt the skin barrier function. ${ }^{11}$ In addition, bath basins and water can contain pathogens related to hospital-acquired infections. ${ }^{12-14}$

In 1990, the bag bath concept was developed, predominantly to tackle the shortcomings related to skin integrity and hygiene..$^{15}$ The bag bath concept consisted of a number of non-disposable washcloths put in a bag together with an amount of no-rinse cleansing fluid diluted in water. Washing without water evolved from the bag bath concept in 1994, and differs from this concept because it is an all-in-one (i.e. no separate water or cleansing fluid required) and completely disposable solution. 


\section{Chapter 3}

Washing without water is claimed to offer several advantages compared to the traditional bed bath with water and soap. First of all, because water, soap, towels and wash basins are not needed, the negative effects on skin integrity and hygiene associated with these materials are eliminated.15, 16 It is claimed that washing without water even has a positive influence on skin condition. ${ }^{17}$ In addition, some authors claim that washing without water is less costly and less time-consuming than the traditional bed bath,16, 18, 19 which is relevant at an organizational level, for the management of health care institutions. Finally, washing without water is said to decrease physical and emotional strain and increase satisfaction among both patients and caregivers. ${ }^{20}$ Some of these claimed outcomes are based on unpublished studies.17, 20 It is unclear whether these are supported by scientific evidence.

Although patient hygiene is a core nursing responsibility, guiding evidence regarding skin cleansing practices, such as washing without water is missing. ${ }^{7,21}$ Such evidence could contribute to the adoption of effective skin cleansing practices in health care. At the moment, it is unclear whether washing without water is an effective skin cleansing practice and therefore a relevant alternative for bathing patients. Therefore, the objective of this systematic review is to assess the effectiveness of washing without water in comparison to the traditional bed bath for the full body wash of immobile patients.

\section{METHODS}

\section{Search strategy}

A systematic literature search was executed in CINAHL, Embase, MEDLINE and PUBMED to collect controlled trials about the effectiveness of the washing without water concept. Studies published before 1995 were not included in the literature search, because only in that year the first article about the bag bath concept was published. Since the washing without water concept does not yet have a commonly accepted generic name, many combinations of adjectives and nouns were used that could represent the concept. Search terms were determined based on the key terms used in literature about washing without water to describe the concept. Identical terms as well as synonyms were used. Furthermore, no specific search terms regarding health care setting, type of patients or outcomes were included as we were interested in all effectiveness outcomes of washing without water for immobile patients in all settings. The search terms and filters used in the different databases are shown in appendix 1. No language limiters were applied in any of the searches. The database search was executed at two points in time. The first search was performed in May, 2015, and in February, 2016, the search was updated. In the second search of February, 2016, only studies published after the date of the first search were retrieved from the databases. Both database searches consisted of two search runs. In the first search run, only studies labeled as a trial were obtained by using the trial filter. The 
second search run combined the search terms of the first search run with the search term 'trial' using the Boolean operator 'AND' and without using the trial filter to ensure that all trials were included in the search results. Additional studies were identified by screening references and by citation searching of the articles screened for full text. Finally, the list of studies was presented to experts in the field of washing without water to ensure that all relevant studies were included. These experts included an author of a previous study related to washing without water, and an infection prevention specialist who is knowledgeable about washing without water.

\section{Study selection}

Studies were eligible if (1) the study designs included outcomes of washing without water products developed for the full body wash compared to the traditional bed bath, and (2) they were controlled trials. Conversely, studies about products specifically developed for other purposes than the regular body wash, such as incontinence care or disinfection, were excluded. Interventions that are related to washing without water but are excluded in this study are presented in Table 1, together with the reasons for exclusion. Finally, no criteria were determined regarding type of patients, nursing staff or health care settings to collect all evidence on outcomes of washing without water.

Table 1 - Interventions which are related to washing without water but are excluded from this study

\begin{tabular}{ll}
\hline Intervention & Reason for exclusion \\
\hline $\begin{array}{l}\text { Barrier wipes that offer } \\
\text { protection to the skin in } \\
\text { the perineum }\end{array}$ & $\begin{array}{l}\text { Although no water is used, these products are not used for the } \\
\text { full body wash. Instead, these products offer protection to } \\
\text { vulnerable skin. }\end{array}$ \\
$\begin{array}{l}\text { Antibacterial wipes, } \\
\text { such as CHG } \\
\text { (Chlorhexidine } \\
\text { Gluconate) wipes }\end{array}$ & $\begin{array}{l}\text { Although no water is used, these products are not used for the } \\
\text { full body wash but for killing bacteria on the skin and thereby } \\
\text { disinfecting the skin. Some studies were found in which CHG } \\
\text { wipes were compared to washing without water products. These } \\
\text { studies did not include washing with water in the comparison or } \\
\text { were not a controlled trial and therefore were excluded. }\end{array}$ \\
$\begin{array}{l}\text { Original bag bath } \\
\text { concept }\end{array}$ & $\begin{array}{l}\text { When the original bag bath concept is used, non-disposable } \\
\text { washcloths are put in a bag together with a no-rinse lotion } \\
\text { diluted in water. Therefore, water still needs to be used to dilute } \\
\text { the no-rinse solution. Furthermore, it is not a disposable solution. }\end{array}$ \\
$\begin{array}{l}\text { Although no water is used, these products are not prepacked so } \\
\text { that one package includes the materials needed for the full } \\
\text { body wash. Separate wipes are still needed and therefore it is } \\
\text { not an all-in-one solution as described in our definition of } \\
\text { washing without water. }\end{array}$ \\
\hline
\end{tabular}




\section{Study screening and data extraction}

Studies were consecutively screened on title, abstract and full text for eligibility. To reduce bias, titles and abstracts were independently screened by two researchers (first author and MM) until the level of agreement between them exceeded $80 \%$. From that moment, the remaining studies were screened by the first author alone. Studies were recorded as either "include", "exclude" or "uncertain", and disagreements between the researchers were discussed to reach consensus. Studies labelled as "include" or "uncertain" were included in subsequent stages of the screening process. Full text screening was done only by the first author. In case of doubt, the first author discussed the full text with one of the other authors (SZ), after which a collaborative decision was made to include or exclude the study.

Data were extracted from the relevant articles using a data extraction form specifically designed for the current review. This form included items needed for the quality assessment of the eligible studies such as items about the research design, study populations, type of interventions, study aims, statistical analyses, outcomes, and recommendations and limitations. For two articles, which lacked information regarding these items, the particular authors were contacted to retrieve additional data or the study protocol. Only one of these authors agreed to share the protocol.

\section{Methodological quality}

Out of an inventory of several methodological quality assessment tools, the scale of Downs and Black was selected to calculate a total quality score (Table 2).22 This scale is one of the most complete assessment tools and covers most risks of bias as described in the Cochrane Handbook for Systematic Reviews of Interventions. ${ }^{23}$ Furthermore, the Agency of Healthcare Research and Quality (AHRQ) argues that the Downs and Black scale is rigorously developed.22, 24 The original two items regarding blinding of persons receiving the intervention and of persons providing the intervention were not applied because this was considered to be practically impossible with the washing without water intervention. Furthermore, the scoring possibilities for the items related to the sample size and the description of principal confounders were adapted for this research (see Table 2). Two researchers (first author and HB) independently assessed study quality and discussed disagreements to reach consensus. When consensus was not reached, the particular items were discussed with one of the other authors (SZ). Based on the quality domains defined by the $A H R Q$, five items from the Downs and Black scale were selected as representing the most important quality requirements. ${ }^{22}$ These items are related to the study aim description (item 1), statistical tests (item 16), randomization (item 21), intention-totreat (item 23), and statistical power (item 25). In this review, a study was considered to be of high quality if it met all five of these items. If a study did not meet all of these criteria, it was considered to be of moderate quality. 
Table 2 - Quality assessment based on Downs and Black22

\begin{tabular}{|c|c|c|c|c|c|c|}
\hline Items & $\begin{array}{l}\text { Gillis } \\
\text { et } \\
\text { al. }{ }^{26}\end{array}$ & $\begin{array}{c}\text { van } \\
\text { Achterberg } \\
\text { et al. }{ }^{25}\end{array}$ & $\begin{array}{l}\text { Schoon- } \\
\text { hoven et } \\
\text { al. }^{2}\end{array}$ & $\begin{array}{l}\text { Nødde- } \\
\text { skou et } \\
\text { al. }{ }^{28}\end{array}$ & $\begin{array}{l}\text { Lar- } \\
\text { son ef } \\
\text { al. }{ }^{27}\end{array}$ & $\begin{array}{c}\text { Shep- } \\
\text { pard \& } \\
\text { Brenner29 }\end{array}$ \\
\hline \multicolumn{7}{|l|}{ Reporting } \\
\hline $\begin{array}{l}\text { 1. Is the hypothesis } \\
\text { /aim/objective of the } \\
\text { study clearly } \\
\text { described?* } \\
\text { Yes }=1, \text { No }=0\end{array}$ & 1 & 1 & 1 & 1 & 1 & 1 \\
\hline $\begin{array}{l}\text { 2. Are the main } \\
\text { outcomes to be } \\
\text { measured clearly } \\
\text { described in the } \\
\text { introduction or } \\
\text { methods section? } \\
\text { Yes }=1, \text { No }=0\end{array}$ & 1 & 1 & 1 & 1 & 1 & 1 \\
\hline $\begin{array}{l}\text { 3. Are the } \\
\text { characteristics of the } \\
\text { patients included in } \\
\text { the study clearly } \\
\text { described? } \\
\qquad \text { Yes }=1, \text { No }=0\end{array}$ & 1 & 1 & 1 & 1 & 1 & 1 \\
\hline $\begin{array}{l}\text { 4. Are the interventions } \\
\text { of interest clearly } \\
\text { described? } \\
\qquad \text { Yes }=1, \mathrm{No}=0\end{array}$ & 1 & 1 & 1 & 1 & 1 & 1 \\
\hline $\begin{array}{l}\text { 5. Are the distributions } \\
\text { of principal } \\
\text { confounders in each } \\
\text { group of subjects to } \\
\text { be compared clearly } \\
\text { described? } † \\
\text { Yes }=1, \text { No }=0\end{array}$ & 1 & 1 & 1 & 0 & 0 & 0 \\
\hline $\begin{array}{l}\text { 6. Are the main } \\
\text { findings of the study } \\
\text { clearly described? } \\
\qquad \text { Yes }=1, \text { No }=0\end{array}$ & 1 & 1 & 1 & 0 & 1 & 1 \\
\hline $\begin{array}{l}\text { 7. Does the study } \\
\text { provide estimates of } \\
\text { the random variability } \\
\text { in the data for the } \\
\text { main outcomes? } \\
\text { Yes }=1, \text { No }=0\end{array}$ & 1 & 1 & 1 & 1 & 1 & 1 \\
\hline
\end{tabular}




\section{Chapter 3}

\begin{tabular}{|c|c|c|c|c|c|c|}
\hline Items & $\begin{array}{l}\text { Gillis } \\
\text { et } \\
\text { al. }{ }^{26}\end{array}$ & $\begin{array}{c}\text { van } \\
\text { Achterberg } \\
\text { et al. }{ }^{25}\end{array}$ & $\begin{array}{l}\text { Schoon- } \\
\text { hoven et } \\
\text { al. }{ }^{2}\end{array}$ & $\begin{array}{l}\text { Nødde- } \\
\text { skou et } \\
\text { al. }{ }^{28}\end{array}$ & $\begin{array}{l}\text { Lar- } \\
\text { son et } \\
\text { al. }{ }^{27}\end{array}$ & $\begin{array}{c}\text { Shep- } \\
\text { pard \& } \\
\text { Brenner29 }\end{array}$ \\
\hline $\begin{array}{l}\text { 8. Have all important } \\
\text { adverse events that } \\
\text { may be a } \\
\text { consequence of the } \\
\text { intervention been } \\
\text { reported? } \\
\text { Yes }=1, \text { No }=0\end{array}$ & 1 & 1 & 1 & 0 & 0 & 1 \\
\hline $\begin{array}{l}\text { 9. Have the } \\
\text { characteristics of } \\
\text { patients lost to follow- } \\
\text { up been described? } \\
\text { Yes }=1, \text { No }=0\end{array}$ & 1 & 0 & 0 & 0 & 0 & 0 \\
\hline $\begin{array}{l}\text { 10. Have actual } \\
\text { probability values } \\
\text { been reported for the } \\
\text { main outcomes } \\
\text { except where the } \\
\text { probability is less than } \\
0.001 \text { ? } \\
\text { Yes }=1, \text { No }=0\end{array}$ & 1 & 1 & 1 & 0 & 1 & 1 \\
\hline External validity & & & & & & \\
\hline $\begin{array}{l}\text { 11. Were the subjects } \\
\text { asked to participate in } \\
\text { the study } \\
\text { representative of the } \\
\text { entire population from } \\
\text { which they were } \\
\text { recruited? } \\
\text { Yes }=1, \text { No }=0 \text {, } \\
\text { Unable to } \\
\text { determine }=0\end{array}$ & 1 & 1 & 1 & 0 & 0 & 0 \\
\hline $\begin{array}{l}\text { 12. Were those } \\
\text { subjects who were } \\
\text { prepared to } \\
\text { participate, } \\
\text { representative of the } \\
\text { entire population from } \\
\text { which they were } \\
\text { recruited? } \\
\text { Yes }=1 \text {, No =0, } \\
\text { Unable to } \\
\text { determine }=0\end{array}$ & 0 & 0 & 0 & 0 & 1 & 0 \\
\hline
\end{tabular}


Chapter 3

\begin{tabular}{|c|c|c|c|c|c|c|}
\hline Items & $\begin{array}{l}\text { Gillis } \\
\text { et } \\
\text { al. }{ }^{26}\end{array}$ & $\begin{array}{c}\text { van } \\
\text { Achterberg } \\
\text { et al. }{ }^{25}\end{array}$ & $\begin{array}{l}\text { Schoon- } \\
\text { hoven et } \\
\text { al. }^{2}\end{array}$ & $\begin{array}{c}\text { Nødde- } \\
\text { skou et } \\
\text { al. }{ }^{28}\end{array}$ & $\begin{array}{l}\text { Lar- } \\
\text { son et } \\
\text { al. }{ }^{27}\end{array}$ & $\begin{array}{c}\text { Shep- } \\
\text { pard \& } \\
\text { Brenner29 }\end{array}$ \\
\hline $\begin{array}{l}\text { 13. Were the staff, } \\
\text { places, and facilities } \\
\text { where the patients } \\
\text { were treated } \\
\text { representative of the } \\
\text { treatment the majority } \\
\text { of patients receive? } \\
\text { Yes }=1, \text { No }=0 \text {, } \\
\text { Unable to } \\
\text { determine }=0\end{array}$ & 1 & 1 & 1 & 1 & 1 & 0 \\
\hline \multicolumn{7}{|l|}{ Internal validity - bias } \\
\hline $\begin{array}{l}\text { 14. If any of the results } \\
\text { of the study were } \\
\text { based on "data } \\
\text { dredging", was this } \\
\text { made clear? } \\
\text { Yes }=1, \text { No }=0 \text {, } \\
\text { Unable to } \\
\text { determine }=0\end{array}$ & 0 & 1 & 1 & 1 & 0 & 0 \\
\hline $\begin{array}{l}\text { 15. Is the time period } \\
\text { between the } \\
\text { intervention and } \\
\text { outcome the same for } \\
\text { cases and controls? } \\
\text { Yes }=1, \text { No }=0 \text {, } \\
\text { Unable to } \\
\text { determine }=0\end{array}$ & 1 & 1 & 1 & 1 & 1 & 1 \\
\hline $\begin{array}{l}\text { 16. Were the statistical } \\
\text { tests used to assess the } \\
\text { main outcomes } \\
\text { appropriate?* } \\
\text { Yes }=1, \text { No }=0 \text {, } \\
\text { Unable to } \\
\text { determine }=0\end{array}$ & 1 & 1 & 1 & 0 & 0 & 0 \\
\hline $\begin{array}{l}\text { 17. Was compliance } \\
\text { with the intervention } \\
\text { reliable? } \\
\qquad \begin{array}{l}\text { Yes }=1, \text { No }=0, \\
\text { Unable to } \\
\text { determine }=0\end{array}\end{array}$ & 0 & 1 & 1 & 1 & 1 & 0 \\
\hline
\end{tabular}


Chapter 3

\begin{tabular}{lcccccc}
\hline Items & $\begin{array}{c}\text { Gillis } \\
\text { et } \\
\text { al.26 }\end{array}$ & $\begin{array}{c}\text { van } \\
\text { Achterberg } \\
\text { et al.25 }\end{array}$ & $\begin{array}{c}\text { Schoon- } \\
\text { hoven et } \\
\text { al. }{ }^{2}\end{array}$ & $\begin{array}{c}\text { Nødde- } \\
\text { skou et } \\
\text { al. } 28\end{array}$ & $\begin{array}{c}\text { Lar- } \\
\text { son et } \\
\text { al. }{ }^{27}\end{array}$ & $\begin{array}{c}\text { Shep- } \\
\text { pard \& } \\
\text { Brenner } 29\end{array}$ \\
\hline $\begin{array}{l}\text { 18. Were the main } \\
\text { outcome measures }\end{array}$ & 1 & 1 & 1 & 0 & 0 & 0 \\
$\begin{array}{c}\text { used accurate? } \\
\text { Yes }=1, \text { No }=0,\end{array}$ & & & & & & \\
Unable to \\
determine $=0$
\end{tabular}

Internal validity - confounding (selection bias)

19. Were patients in different intervention

groups or were the cases and controls recruited from the same population?

Yes $=1, \mathrm{No}=0$,

Unable to

determine $=0$

20. Were the study subjects in different intervention groups or were the cases and controls recruited from the same population?

Yes $=1, \mathrm{No}=0$,

Unable to

determine $=0$

21. Were study

1

0

0

1

1

subjects randomized

to intervention

groups?*

Yes $=1, \mathrm{No}=0$,

Unable to

determine $=0$

22. Was the

randomized

intervention

assignment

concealed from both

patients and health

care staff untill

recruitment 


\begin{tabular}{|c|c|c|c|c|c|c|}
\hline Items & $\begin{array}{l}\text { Gillis } \\
\text { et } \\
\text { al. }{ }^{26}\end{array}$ & $\begin{array}{c}\text { van } \\
\text { Achterberg } \\
\text { et al. }{ }^{25}\end{array}$ & $\begin{array}{l}\text { Schoon- } \\
\text { hoven et } \\
\text { al. }^{2}\end{array}$ & $\begin{array}{l}\text { Nødde- } \\
\text { skou et } \\
\text { al. }^{28}\end{array}$ & $\begin{array}{l}\text { Lar- } \\
\text { son et } \\
\text { al. }{ }^{27}\end{array}$ & $\begin{array}{c}\text { Shep- } \\
\text { pard \& } \\
\text { Brenner29 }\end{array}$ \\
\hline $\begin{array}{l}\text { was complete } \\
\text { and irrevocable? } \\
\text { Yes }=1, \text { No }=0 \\
\text { Unable to } \\
\text { determine }=0\end{array}$ & & & & & & \\
\hline $\begin{array}{l}\text { 23. Was there ade- } \\
\text { quate adjustment for } \\
\text { confounding in the } \\
\text { analyses from which } \\
\text { the main findings were } \\
\text { drawn?* }\end{array}$ & 1 & 1 & 1 & 1 & 0 & 1 \\
\hline $\begin{array}{l}\text { Yes }=1, \text { No }=0 \\
\text { Unable to } \\
\text { determine }=0\end{array}$ & & & & & & \\
\hline $\begin{array}{l}\text { 24. Were losses of } \\
\text { patients to follow-up } \\
\text { taken into account? } \\
\text { Yes }=1, \text { No }=0, \\
\text { Unable to } \\
\text { determine }=0\end{array}$ & 1 & 1 & 1 & 0 & 0 & 0 \\
\hline \multicolumn{7}{|l|}{ Power } \\
\hline $\begin{array}{l}\text { 25. Did the study have } \\
\text { sufficient power to } \\
\text { detect a clinically } \\
\text { important effect } \\
\text { where the probability } \\
\text { value for a difference } \\
\text { being due to chance } \\
\text { is less than } 5 \% \text { ? }^{*} \\
\text { Yes }=1, \text { No }=0\end{array}$ & 0 & 1 & 1 & 0 & 0 & 0 \\
\hline Total quality score & $20 / 25$ & $21 / 25$ & $21 / 25$ & $14 / 25$ & $13 / 25$ & $12 / 25$ \\
\hline $\begin{array}{l}\text { Important quality } \\
\text { items met }\end{array}$ & $4 / 5$ & $5 / 5$ & $5 / 5$ & $3 / 5$ & $1 / 5$ & $2 / 5$ \\
\hline
\end{tabular}

\section{Excluded original items for this study:}

- Was an attempt made to blind study subjects to the intervention they have received?

- Was an attempt made to blind those measuring the main outcomes of the intervention?

Reason for exclusion: it is practically impossible to blind study subjects and those who measure outcomes for the washing without water intervention.

*Important quality items that needed to be met to be considered as a study of high quality †The original answer possibilities (5: Yes $=2$, Partially $=1$, No $=0$ and $25:<n 1=0, n 1-n 2=1, n 3-$ $\mathrm{n} 4=2, \mathrm{n} 5-\mathrm{n} 6=3, \mathrm{n} 7-\mathrm{n} 8=4, \mathrm{n} 8+=5$ ) have been adapted 


\section{Data synthesis and analysis}

An overview of the evidence related to all washing without water outcomes was made. We categorized findings on the effectiveness of washing without water in (1) physiological outcomes related to hygiene and skin condition, (2) stakeholder-related outcomes, and (3) organizational outcomes. All results of the studies of moderate or high quality are presented.

\section{RESULTS}

\section{Search results}

Figure 1 shows the results of the screening process. The numbers of studies mentioned in the figure are the totals of the articles retrieved in May, 2015, and February, 2016. Of the 1,830 unique studies that were retrieved from the electronic database search, ultimately, six studies were included in this review. The main reason for exclusion was that the intervention in the particular studies was not a washing without water product.

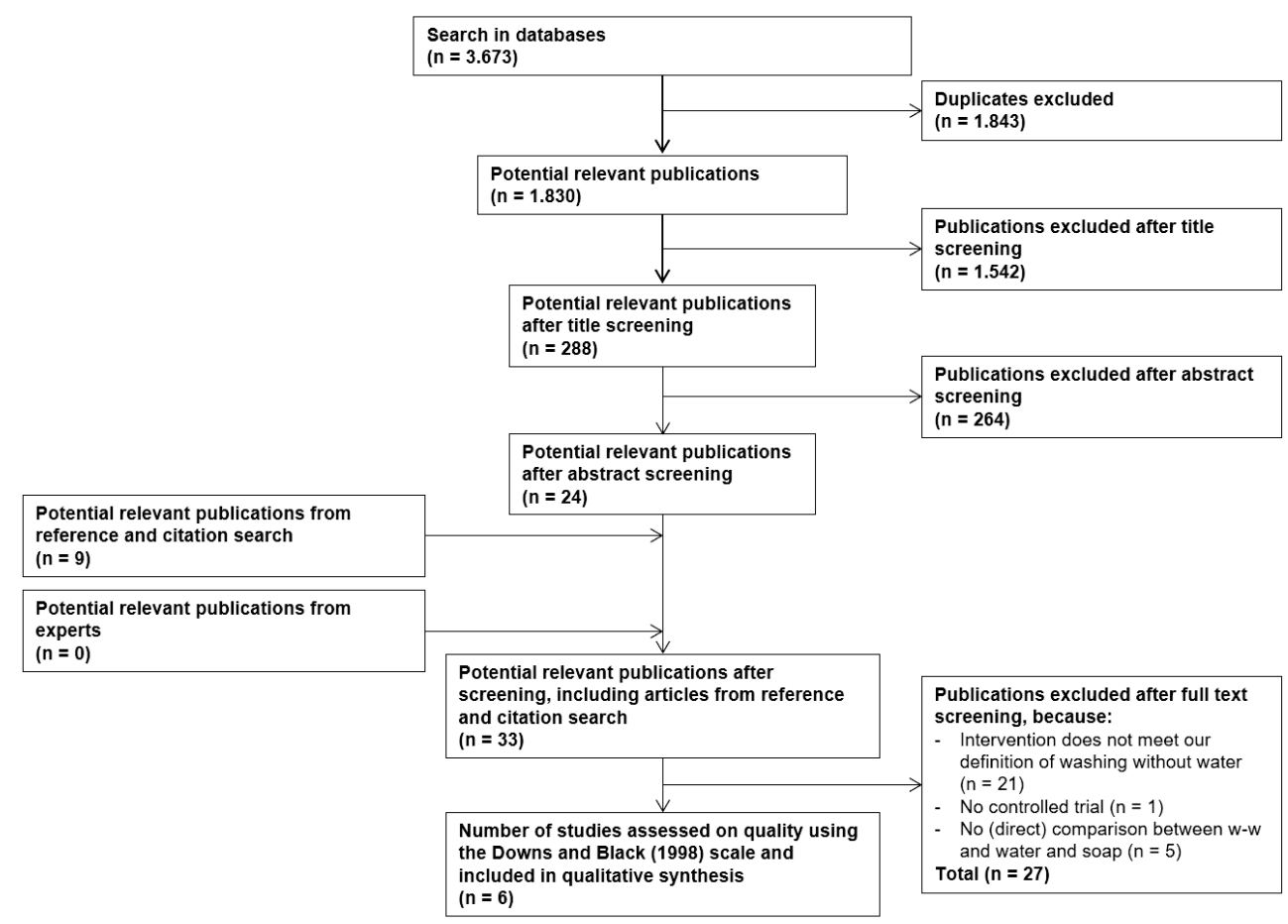

Figure 1 - PRISMA flow diagram. Legend: A total of 6 studies were used to inform the findings for this manuscript. None of the studies labeled as "uncertain" based on title or abstract screening turned out to be eligible. Flow diagram adapted from Moher, Liberati, Tetzlaff, Altman, and THE PRISMA Group ${ }^{37}$ 


\section{Study characteristics and quality}

Information regarding the characteristics of all included studies is provided in Table 3. The studies employed different study designs, including three randomized controlled trials. 2, 25, 26 The studies of Schoonhoven et al. and van Achterberg et al. were based on the same research data.2, 25 Two other studies applied a controlled cross-over design over two consecutive days in which all subjects received the traditional bed bath on 1 day and a bed bath with a washing without water product on the other day. 27,28 The order in which subjects received the different bed baths was randomized in only one of these studies. ${ }^{28}$ The last study had a quasi-experimental design. ${ }^{29}$

Table 3 - Study characteristics

\begin{tabular}{|c|c|c|c|}
\hline $\begin{array}{l}\text { Author, year and } \\
\text { country }\end{array}$ & Study design & $\begin{array}{l}\text { Setting and sample population at } \\
\text { baseline }\end{array}$ & $\begin{array}{l}\text { Study } \\
\text { duration }\end{array}$ \\
\hline $\begin{array}{l}\text { Gillis et al. }{ }^{26} \\
\text { Belgium }\end{array}$ & $\begin{array}{l}\text { Cluster } \\
\text { controlled trial }\end{array}$ & $\begin{array}{l}\text { Institutionalized long-term care } \\
6 \text { wards in } 2 \text { nursing homes } \\
163 \text { Residents with an average age } \\
\text { of } 84.9\end{array}$ & 12 weeks \\
\hline $\begin{array}{l}\text { van Achterberg et } \\
\text { al. } 25 \\
\text { The Netherlands }\end{array}$ & $\begin{array}{l}\text { Cluster } \\
\text { controlled trial }\end{array}$ & $\begin{array}{l}\text { See Schoonhoven et al. }{ }^{2} \text { for setting } \\
\text { and sample as the same dataset } \\
\text { was used }\end{array}$ & 6 weeks \\
\hline $\begin{array}{l}\text { Schoonhoven et } \\
\text { al. }{ }^{2} \\
\text { The Netherlands }\end{array}$ & $\begin{array}{l}\text { Cluster } \\
\text { controlled trial }\end{array}$ & $\begin{array}{l}\text { Institutionalized long-term care } \\
56 \text { wards in } 22 \text { nursing homes } \\
500 \text { Residents: } \\
61.6 \% \text { Were diagnosed with } \\
\text { dementia; } \\
\text { average age of } 82.4 \\
275 \text { Nurses }\end{array}$ & 6 weeks \\
\hline $\begin{array}{l}\text { Nøddeskou et al. }{ }^{28} \\
\text { Denmark }\end{array}$ & $\begin{array}{l}\text { Randomized } \\
\text { controlled } \\
\text { cross-over trial }\end{array}$ & $\begin{array}{l}\text { One hospital } \\
65 \text { Patients with an average age of } \\
73 \\
6 \text { Nurses }\end{array}$ & 2 days \\
\hline $\begin{array}{l}\text { Larson et al. }{ }^{27} \\
\text { United States }\end{array}$ & Cross-over trial & $\begin{array}{l}\text { Hospital - Three intensive care units } \\
\text { of one hospital } \\
47 \text { Patients with an average age of } \\
60.7 \\
40 \text { Nurses }\end{array}$ & 2 days \\
\hline $\begin{array}{l}\text { Sheppard \& } \\
\text { Brenner } 29 \\
\text { United States }\end{array}$ & $\begin{array}{l}\text { Controlled time } \\
\text { series trial }\end{array}$ & $\begin{array}{l}\text { Institutionalized long-term care } \\
\text { One nursing home } \\
35 \text { Residents: } \\
56.25 \% \text { Were cognitively impaired; } \\
\text { average age of } 85.17 \\
11 \text { Nurses }\end{array}$ & 6 weeks \\
\hline
\end{tabular}




\section{Chapter 3}

Moreover, research settings and populations also differed between the studies. Two studies were conducted in a hospital,27, 28 and four took place in institutionalized long-term care.2, 25, 26, 29 Furthermore, the four studies conducted in institutionalized long-term care only included residents aged 65 years or older (the average ages ranged from 82.4 to 85.17 years), whereas the two studies situated in hospitals also included patients younger than 65 years (leading to average ages of 73 and 60.7 years).27, 28 Information regarding cognitive impairment as a feature of the study population was only reported in three studies, $2,25,29$ the details of which are mentioned in Table 3. The study by Nøddeskou et al. excluded cognitively impaired patients, ${ }^{28}$ whereas the other two studies did not mention cognitive impairment as an exclusion criteria nor did they report on the presence of such patients. ${ }^{26,} 27$

Information about the quality assessment of the studies can be found in Table 2. Only the studies by Schoonhoven et al. and van Achterberg et al. met all five of the primary quality items and were therefore considered to be of high quality. ${ }^{2,} 25$ These studies also received the highest total quality scores of all included studies (21/25). The other four studies were considered to be of moderate quality. Of these four, the study of Gillis et al. did obtain a high total score, but was not considered to be of high quality as it did not meet all of the five primary quality items (4/5). ${ }^{26}$ Due to the small number of studies, all studies of moderate to high quality were included in the data synthesis. Furthermore, because only a limited number of studies was found and the reported outcomes varied considerably between studies, it was not possible to assess the risk of bias across studies nor to conduct a meta-analysis.

Table 4 shows a complete overview of the results per included study. Skin hydration, ${ }^{26}$ any skin abnormalities, significant skin lesions, ${ }^{2}$ reduction in microbial counts, ${ }^{27}$ and skin dryness ${ }^{29}$ were categorized as physiological outcomes. Resident satisfaction, 2, 29 nurse satisfaction, 2, 27-29 bathing completeness, ${ }^{25}$ and resistance during bathing ${ }^{2}$ were regarded to be stakeholder-related outcomes. Finally, organizational outcomes were costs (including staff wages and material costs), 2, 27, 28 time of a bed bath, 27,28 and the quality score of the bed bath. ${ }^{27,} 28$ 


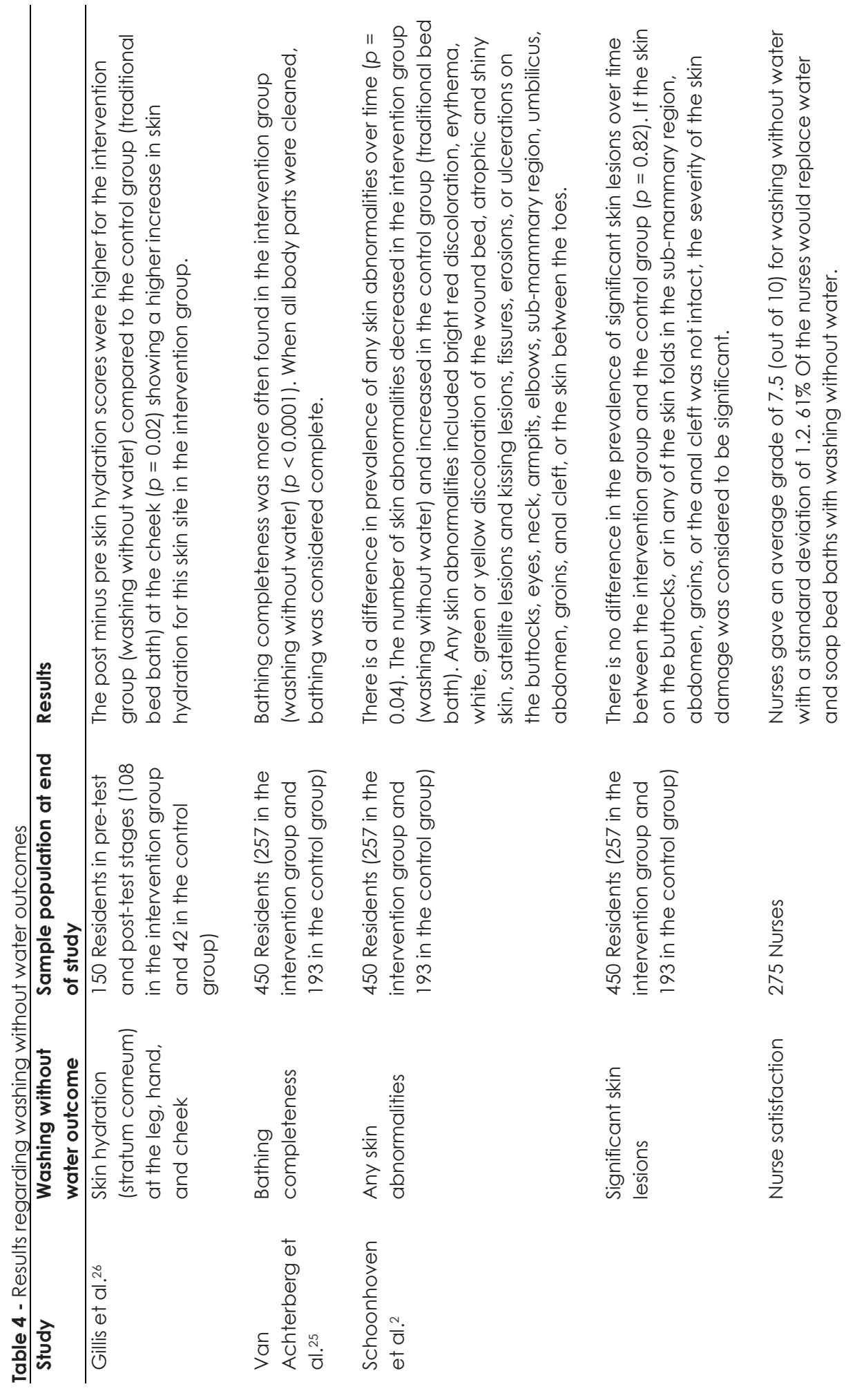




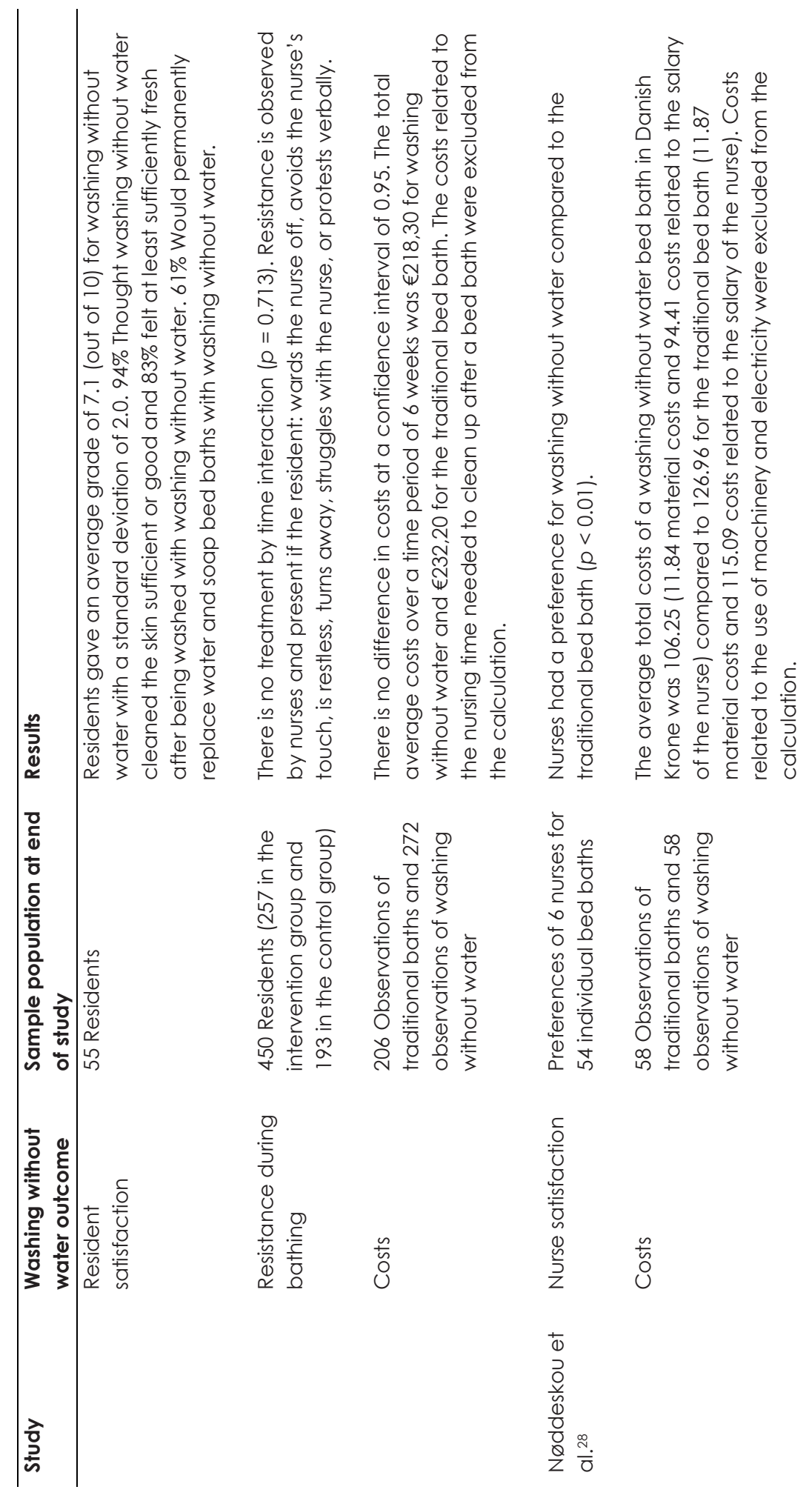




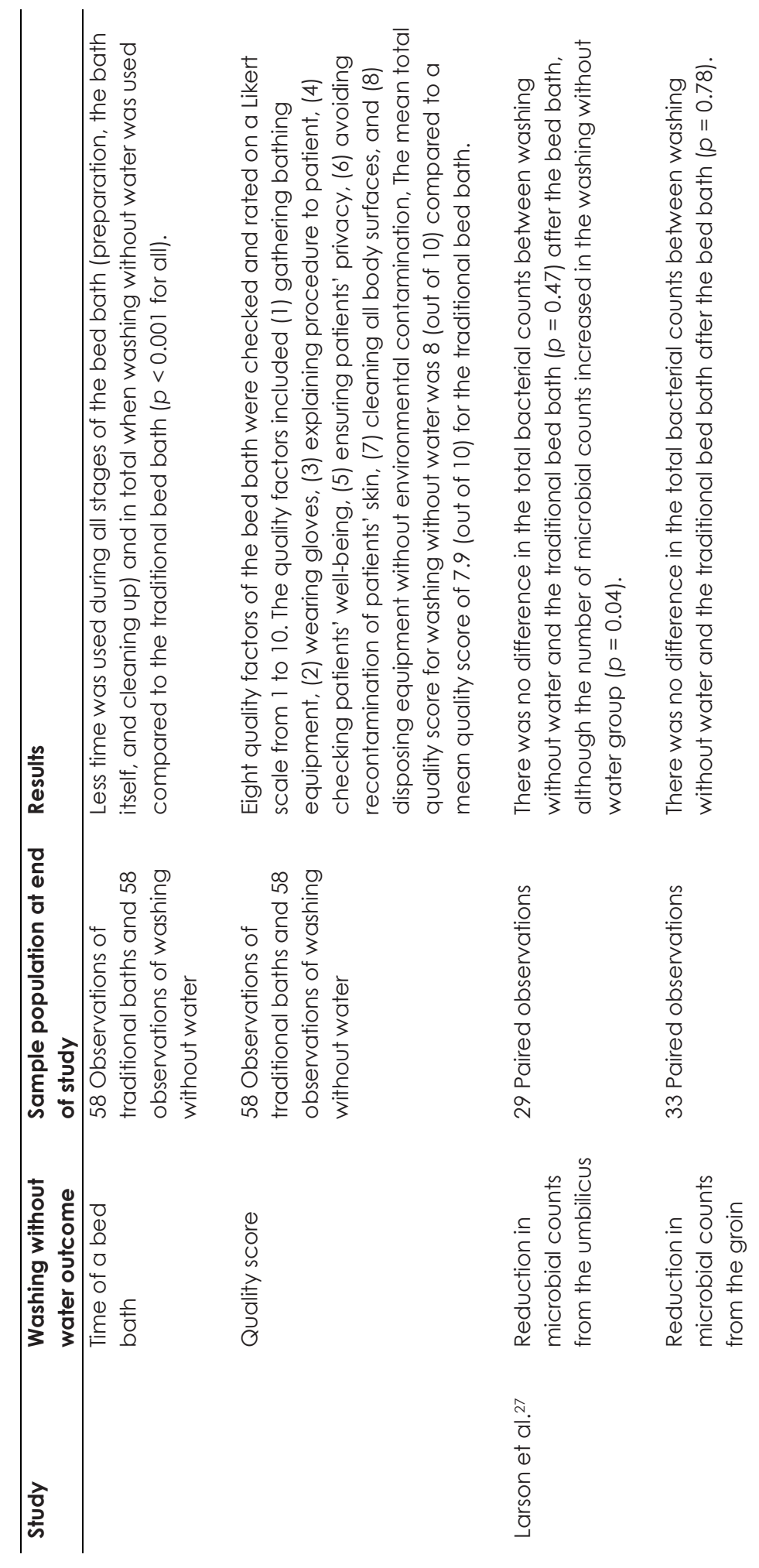




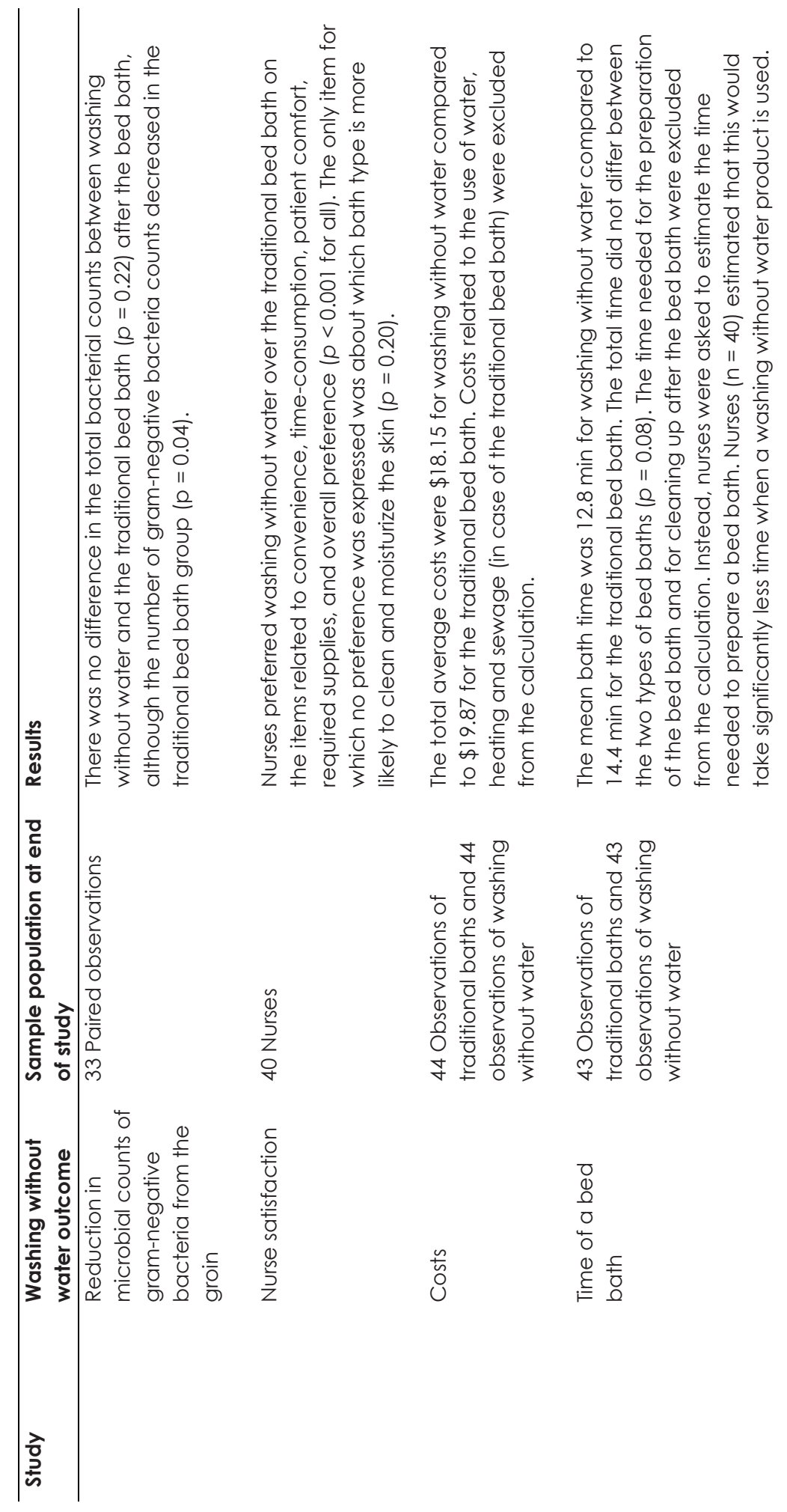




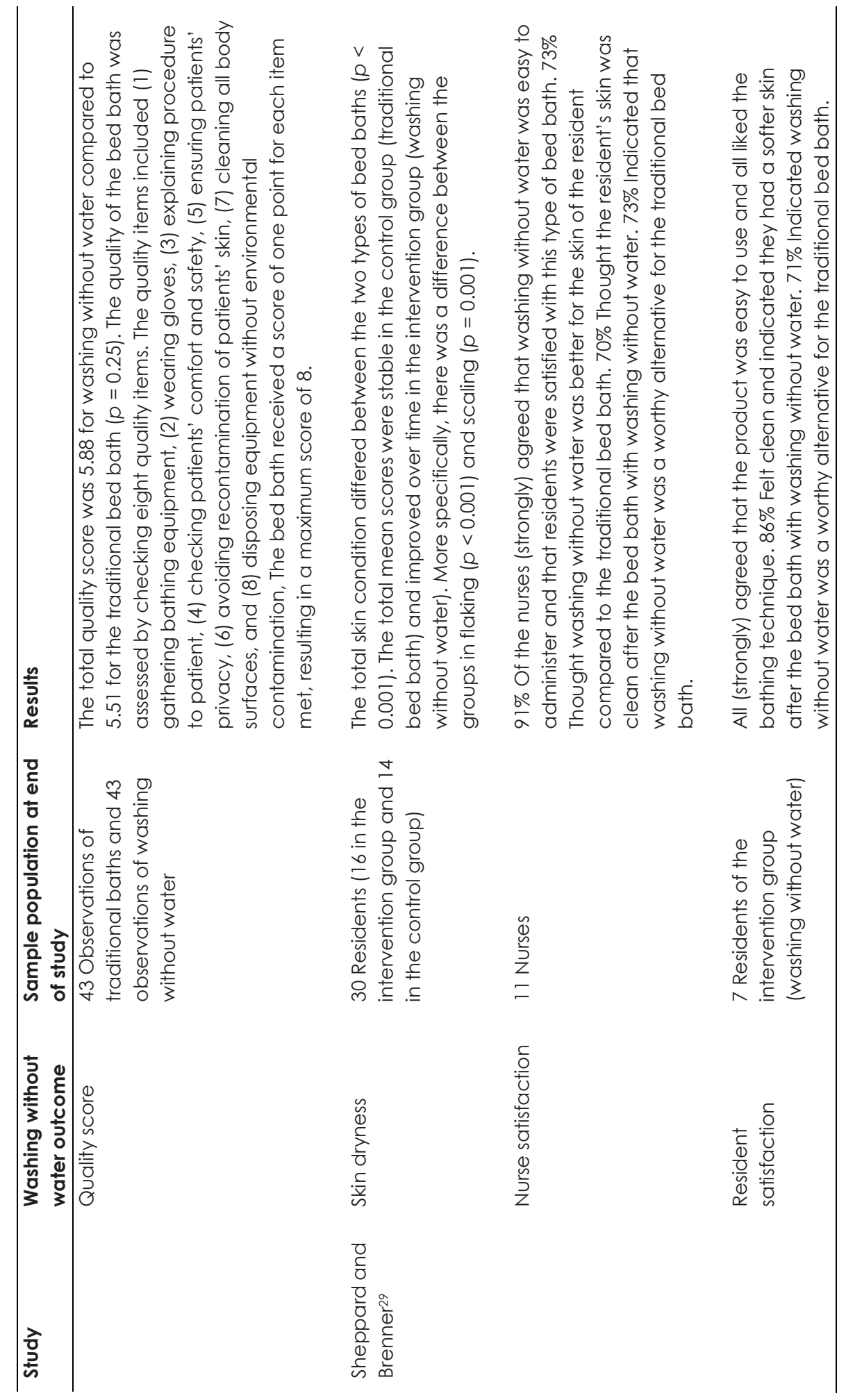




\section{Results of physiological washing without water outcomes}

Outcomes related to hygiene and skin condition are regarded to be physiological outcomes of washing without water in this review. The high quality study by Schoonhoven et al. measured outcomes related to residents' skin condition, being any skin abnormalities and significant skin lesions. ${ }^{2}$ If the skin of certain parts of the body was not intact, the severity of the skin lesion was considered to be significant, whereas skin abnormalities included, amongst others, erythema and skin discoloration. A significant difference in the prevalence of skin abnormalities over time (6 weeks) was observed between the washing without water group, in which the prevalence decreased, and the traditional bed bath group, in which the prevalence increased. However, no significant difference was found regarding the occurrence of significant skin lesions over time. Other findings related to the skin condition were presented in the studies of moderate quality from Sheppard and Brenner and Gillis et al. 26,29 The results of Sheppard and Brenner showed a significant difference between the washing without water group and the traditional bed bath group regarding skin dryness in favor of washing without water. ${ }^{29}$ Furthermore, Gillis et al. concluded that skin hydration at the cheek increased significantly more when washing without water was adopted compared to the traditional bed bath. ${ }^{26}$ No differences were found for skin hydration at hands or legs. Physiological outcomes related to hygiene were only reported in the study of moderate quality by Larson et al., where no significant differences were found. ${ }^{27}$

\section{Results of stakeholder-related washing without water outcomes}

Next to physiological outcomes, stakeholder-related outcomes are considered to be important in this review. In a study of high quality, Schoonhoven et al. reported a resident satisfaction score of 7.1 (out of 10) and a nursing staff satisfaction score of 7.5 for washing without water. ${ }^{2}$ However, they did not compare satisfaction regarding washing without water with the level of satisfaction regarding the traditional bed bath. Also Sheppard and Brenner studied resident and nurse satisfaction without comparing the level of satisfaction between the two bathing methods in their study of moderate quality. ${ }^{29}$ A comparison of nursing staff satisfaction was made in the studies of moderate quality by Larson et al. and Nøddeskou et al. ${ }^{27,} 28$ Nursing staff in both studies significantly preferred washing without water over the traditional bed bath. The high quality study outcomes "bathing completeness" and "resistance during bathing" are also considered to be stakeholder-related outcomes in this review. Bathing completeness was found to be significantly higher for washing without water compared to the traditional bed bath. ${ }^{25}$ Finally, no significant treatment by time interaction was found regarding resistance during bathing, which means that residents bathed with washing without water did not show more or less resistance compared to residents bathed the traditional way. ${ }^{2}$ 


\section{Results of organizational washing without water outcomes}

Finally, several findings related to organizational outcomes of washing without water compared to the traditional bed bath were reported. The only study of high quality that reported on costs did not find a significant difference in the total average costs over a time period of 6 weeks between washing without water and the traditional bed bath. ${ }^{2}$ In two other studies of moderate quality, the average total costs of a bed bath were reported to be DKK 106.25 and $\$ 18.15$ for washing without water and DKK 126.96 and $\$ 19.87$ for the traditional bed bath, respectively. ${ }^{27,} 28$ An important costs component is the nursing time spent on the bed bath. The complete time from preparation of the bed bath to clean-up was only studied in one study of moderate quality in which a significant difference was found both in the total bed bath time and in the time of all individual stages of the bed bath, which were all shorter for washing without water $(p<0.001) .28$ No significant time difference was found in the study of moderate quality of Larson et al., who did not include the time needed for preparation and clean up. ${ }^{27}$ Finally, the quality of the bed bath was measured in two studies of moderate quality in which no difference between the two bathing methods was found. 28,29

\section{DISCUSSION}

Evidence on outcomes of washing without water compared to the traditional bed bath is scarce. Six controlled trials on the effects of washing without water were found, of which only two were regarded to be of high quality.2, 25 Moreover, these two studies were of the same research group and used the same dataset for the analysis. Nevertheless, the results of the few studies that have been conducted show that washing without water is not inferior to, and on some outcomes even outperforms the traditional bed bath. Washing without water performed significantly better than the traditional bed bath with respect to skin abnormalities, ${ }^{2}$ skin dryness/hydration, ${ }^{26,} 29$ nurse satisfaction, 27, 28 and bathing completeness, ${ }^{25}$ of which only skin abnormalities and bathing completeness have been studied in studies of high quality. Furthermore, one study found a time difference, ${ }^{28}$ whereas the time of the bed bath did not differ in another study. ${ }^{27}$ Both studies were assessed to be of moderate quality but only the former included the total time of a bed bath from preparation to cleanup. In addition, no significant differences between washing without water and the traditional bed bath were found for significant skin lesions, resistance during bathing or costs, in one of the studies of high quality. ${ }^{2}$ Furthermore, studies of moderate quality did not find significant differences for microbial counts or the quality of the bed bath. ${ }^{27,} 28$

\section{Reflection on findings}

Reflecting on the evidence on washing without water, we yield some noticeable findings. First, none of the studies included all cost components in the cost calculation. For example, Schoonhoven et al. did not include the time that nursing staff spent cleaning up after the bed bath. ${ }^{2}$ Because nursing time is an important cost 


\section{Chapter 3}

component related to the bed bath, substantial costs may not have been included in the cost calculation. Second, patient satisfaction between washing without water and the traditional bed bath has not been compared in any of the included studies, which is interesting given the growing attention for patient-centered care. The Institute of Medicine defines patient-centered care as care that suits patients' needs and preferences. ${ }^{30}$ Consequently, patient-centered care is argued to contribute to patient satisfaction. ${ }^{31}$ The scarce evidence regarding the effect of washing without water on patient satisfaction could be explained by the output-oriented management approach in health care. From an output perspective, the bed bath is merely provided to reach cleanliness. ${ }^{32}$ However, evidence on the effectiveness of washing without water regarding hygiene is also still scarce, as it is measured in only one of the included studies. ${ }^{27}$

Finally, washing without water is increasingly used in health care institutions, while the evidence on washing without water in general, and on hygiene and patient satisfaction in particular, is limited. Thoma-Lürken et al. also conclude that the effectiveness of many implemented health care interventions, such as washing without water, need to be further assessed. ${ }^{1}$ One could take the argument of Feo and Kitson, 32 that bathing is often perceived as a low-priority nursing task, to explain the lack of evidence regarding washing without water. ${ }^{19}, 33$ However, the number of studies about bathing and hygiene that we encountered while conducting this review demonstrates that the nursing field is highly interested in patient hygiene. Therefore, we encourage nurse researchers to actively cooperate with clinical researchers to conduct more trials of high quality that inform nursing staff on how to provide the best possible care, and consequently, to upgrade the perceived value of fundamental care. ${ }^{32}$

\section{Research implications}

The scarce evidence regarding washing without water in general, and regarding washing without water outcomes related to costs, hygiene and patient satisfaction in particular, calls for additional research. Although costs were measured in half of the included studies, 2, 27, 28 not all costs were included in the measurements. A complete cost overview would contribute to the burden of proof regarding the costeffectiveness of both bathing methods. Furthermore, the only study that reported on hygiene outcomes did not find a significant difference in total microbial counts from the groin or the umbilicus after the bed bath between washing without water and the traditional bed bath. ${ }^{27}$

From a patient-centered care perspective, patient-related outcomes should be studied more elaborately, including patient satisfaction, patient experiences, and patients' value perceptions regarding washing without water. According to Berwick, patient experiences lie at the heart of quality of care. ${ }^{34}$ The focus on patient experiences is consistent with patient-centered care and is likely to contribute to patient satisfaction. ${ }^{31}$ Patient experiences are not only related to patient satisfaction and to quality of care, but also to the patient's perceptions of the value of care. ${ }^{35}$ For 
washing without water, this means that patients evaluate the value of the washing without water bed bath based on their experiences with it.

However, the patient is not the only stakeholder involved in the bed bath. The nursing staff is another important stakeholder group. Nursing staff may decide whether or not to use a washing without water product without providing the patient a choice. Moreover, family members are important stakeholders as they generally are highly involved in health care, especially when patients are old and vulnerable. Different stakeholders have diverse perceptions of value depending on their individual needs and contexts. ${ }^{36}$ For example, patients might derive value from the experience of having soft skin, whereas the nursing staff might derive value from giving an emotionally and physically burden-free bed bath. Hence, not only should future research on washing without water consider bed bath experiences of patients, but it should also consider bed bath experiences of other stakeholders, such as nursing staff and family. Moreover, it should take into account stakeholders' different value perceptions of washing without water.

Based on the importance of experiences and value perceptions of patients, nursing staff, and family members, another recommendation for future research is to study the relationship between the outcomes of washing without water which can be measured objectively, on the one hand, and stakeholders' experiences and value perceptions on the other hand (e.g. by adopting a mixed method approach). For example, one of the outcomes of washing without water is that it is more timeeffective compared to the traditional bed bath. We do not know whether this has a positive or a negative effect on the patients' or nursing staff's experiences of the bed bath. On the one hand, a shorter bed bath can be less burdensome and therefore positively affect experiences and value perceptions of patients and nursing staff. Moreover, time-effectiveness of washing without water might be positively related to bathing completeness as argued by van Achterberg et al. ${ }^{25}$ On the other hand, patients and nursing staff might perceive a shorter bed bath as being impersonal and therefore a reduction in bed bath quality. ${ }^{2}$ By elaborately examining patients' and nursing staff's experiences, valuable information can be obtained about the importance of washing without water outcomes for the experience and the value perceptions of patients and nursing staff.

Finally, this review points to two methodological implications. First, future research is needed to come to credible generalizations about the effectiveness of washing without water. The two studies of high quality were conducted in an institutionalized long-term care setting. Therefore, especially for outcomes that are unique to these studies, such as bathing completeness and skin abnormalities, it would be interesting to study the outcomes of these studies in other settings as well.2, 25 Furthermore, while three of the included studies included patients with cognitive impairments, 2, 25, 29 and one study excluded cognitively impaired patients, ${ }^{28}$ two studies did not report on the presence of such patients. ${ }^{26,} 27$ Hence, it is unclear whether results of the studies that excluded cognitively impaired patients are generalizable to settings where these patients are present. The inclusion of cognitively 


\section{Chapter 3}

impaired patients in half of the included studies demonstrates that washing without water might be particularly valuable for this patient population. Second, many different outcome measures have been used in the included studies and the internal validity is not always described. The use of outcome measures with high internal validity would enhance the reliability of guiding evidence related to bathing practices, such as washing without water.

\section{Limitations of this review}

Two limitations of this study are related to the quality assessment of eligible studies adopted for this review. First of all, the Downs and Black quality checklist does not provide a cut-off value that can be used to distinguish high quality studies from studies of lower quality. ${ }^{22}$ Hence, we selected five key criteria that needed to be met to be qualified as a study of high quality. The selection of other key criteria might have led to different quality assessment results. However, since all results of studies of moderate to high quality were included, we did not miss important outcomes regarding the effectiveness of washing without water. The second limitation regarding the quality assessment is that we did not assess the risk of reporting bias. We only asked authors of eligible studies for the study protocol in cases where information that we needed for the quality assessment was missing. We did not compare the study protocols of all included studies with the published results and therefore, were unable to assess whether authors did report on all intended outcomes. Moreover, the classification of outcomes as physiological, stakeholder-related or organizational is based on our own judgment. Although this classification is arbitrary, we did not miss important outcomes as all reported outcomes are included in one of the outcome groups.

To our best knowledge, the current study is the first systematic review of washing without water. This review provides a complete overview of the evidence on washing without water as we included all controlled trials irrespective of the outcomes studied, type of patients or health care setting. Our eligibility criteria are based on a restricted definition of washing without water, excluding bathing concepts and products such as the bag bath concept and antibacterial wipes. Furthermore, studies that did not adopt a controlled trial design were excluded from this review. Although some may consider that our chosen criteria limit the search results, we consider the criteria to be a strength of this study. By excluding concepts such as the bag bath, we assured that the results were related to the exact same intervention, being washing without water. In our opinion, the inclusion of related but distinct concepts would have clouded the results. Moreover, we argue that the inclusion of non-controlled trials would have weakened the results.

\section{CONCLUSIONS}

Although only a few studies related to washing without water in comparison to the traditional bed bath were found, this review offers valuable evidence to health care institutions by indicating that washing without water can be seen as a worthy 
alternative to the traditional bed bath. Because the two studies of high quality were conducted in an institutionalized long-term care setting, the evidence is particularly relevant to long-term care institutions. The results show that washing without water does not underperform compared to the traditional bed bath. Additionally, washing without water performs better on some outcomes. Consequently, compared to the traditional bed bath, washing without water might offer more advantages and value to the patient and the nursing staff, but possibly also to other stakeholders such as family members and the management of health care institutions. Especially because the washing without water concept is increasingly used in health care, there is a need for additional research to substantiate the advantages of washing without water. Future research should particularly focus on hygiene outcomes, and on patients', nursing staff's and family members' value perceptions and experiences related to washing without water. 


\section{References}

1. Thoma-Lürken T, Bleijlevens MHC, Lexis MAS, et al. An overview of potential labor-saving and quality-improving innovations in long-term care for older people. Journal of the American Medical Directors Association 2015;16:482-489.

2. Schoonhoven L, van Gaal BGl, Teerenstra S, et al. Cost-consequence analysis of "washing without water" for nursing home residents: a cluster randomized trial. International Journal of Nursing Studies 2015;52:112-120.

3. Gozalo P, Prakash S, Qato DM, et al. Effect of the bathing without a battle training intervention on bathing-associated physical and verbal outcomes in nursing home residents with dementia: A randomized crossover diffusion study. Journal of the American Geriatrics Society 2014;62:797-804.

4. Barsevick A and Llewellyn J. A comparison of the anxiety-reducing potential of two techniques of bathing. Nursing Research 1982;31:22-27.

5. Downey L and Lloyd H. Bed bathing patients in hospital. Nursing Standard 2008;22:3540.

6. Vollman K, Garcia R and Miller L. Interventional patient hygiene: proactive (hygiene) strategies to improve patients' outcomes. AACN News 2005;22:12-16.

7. Cowdell F and Steventon K. Skin cleansing practices for older people: a systematic review. International Journal of Older People Nursing 2015;10:3-13.

8. Lopes JL, Nogueira-Martins LA and de Barros ALBL. Bed and shower baths: comparing the perceptions of patients with acute myocardial infarction. Journal of Clinical Nursing 2012;22:733-740.

9. Hoeffer B, Talerico KA, Rasin J, et al. Assisting cognitively impaired nursing home residents with bathing: effects of two bathing interventions on caregiving. The Gerontologist 2006;46:524-532.

10. Sloane PD, Rader J, Barrick AL, et al. Bathing persons with dementia. The Gerontologist 1995;35:672-678.

11. Voegeli $D$. The effect of washing and drying practices on skin barrier function. Journal of Wound, Ostomy, and Continence Nursing 2008;35:84-90.

12. Marchaim D, Taylor AR, Hayakawa K, et al. Hospital bath basins are frequently contaminated with multidrug-resistant human pathogens. American Journal of Infection Control 2012;40:562-564.

13. Johnson D, Lineweaver L and Maze LM. Patients' bath basins as potential sources of infection: a multicenter sampling study. American Journal of Critical Care 2009;18:3140.

14. Anaissie EJ, Penzak SR and Dignani MC. The hospital water supply as a source of nosocomial infections. Archives of Internal Medicine 2002;162:1483-1492.

15. Skewes SM. Skin care rituals that do more harm than good. American Journal of Nursing 1996:96:33-35.

16. Collins F and Hampton S. The cost-effective use of BagBath: a new concept in patient hygiene. British Journal of Nursing 2003;12:984-990.

17. Knibbe JJ and Knibbe NE. Businesscase verzorgend wassen wassen-zonder-water. Stichting RegioPlus/Platform Zorginnovatie, Zoetermeer. 2006. Available from: http://www.locomotion.nu/doc/innovatiespiegel/BusinesscaseVW.pdf. Accessed 13 Aug 2015.

18. Carruth AK, Ricks D and Pullen P. Bag baths: an alternative to the bed bath. Nursing Management 1995;26:75-78. 
19. Eigsti JE. Innovative solutions: beds, baths, and bottoms: a quality improvement initiative to standardize use of beds, bathing techniques, and skin care in a general critical-care unit. Dimensions of Critical Care Nursing 2011;30:169-176.

20. Knibbe H, Geuze L and Knibbe N. Ergonomische aspecten van wassen-zonder-water voor zorgverleners. $2005 . \quad$ Available from: http://docs.minszw.nl/pdf/92/2005/92_2005_1_9249.pdf. Accessed 13 Aug 2015.

21. Hodgkinson B, Nay R and Wilson J. A systematic review of topical skin care in aged care facilities. Journal of Clinical Nursing 2007;16:129-136.

22. Downs SH and Black N. The feasibility of creating a checklist for the assessment of the methodological quality both of randomised and non-randomised studies of health care interventions. Journal of Epidemiology and Community Health 1998;52:377-84.

23. Cochrane Handbook for Systematic Reviews of Interventions [database on the Internet]. The Cochrane Collaboration. 2011. Available from: www.handbook.cochrane.org. Accessed: $30 \mathrm{Jul} 2015$.

24. West S, King V, Carey TS, et al. Systems to Rate the Strength of Scientific Evidence. Evidence Report/Technology Assessment No. 47 (Prepared by the Research Triangle Institute-University of North Carolina Evidence-based Practice Center under Contract No. 290-97-001 1). AHRQ Publication No. 02-E016. Rockville, MD: Agency for Healthcare Research and Quality; 2002.

25. van Achterberg T, van Gaal BGl, Geense WW, et al. Completeness of assisted bathing in nursing homes related to dementia and bathing method: results from a secondary analysis of cluster-randomised trial data. International Journal of Older People Nursing 2015;11:121-129.

26. Gillis K, Tency I, Roelant E, et al. Skin hydration in nursing home residents using disposable bed baths. Geriatric Nursing 2015:1-5.

27. Larson EL, Ciliberti T, Chantler C, et al. Comparison of traditional and disposable bed baths in critically ill patients. American Journal of Critical Care 2004;13:235-241.

28. Nøddeskou LH, Hemmingsen LE and Hørdam B. Elderly patients' and nurses' assessment of traditional bed bath compared to prepacked single units - randomised controlled trial. Scandinavian Journal of Caring Sciences 2015;29:347-352.

29. Sheppard CM and Brenner PS. The effects of bathing and skin care practices on skin quality and satisfaction with an innovative product. Journal of Gerontological Nursing 2000;26:36-45.

30. Crossing the Quality Chasm: A new health system for the 21 st century. Institute of Medicine. 2001. Available from:

https://iom.nationalacademies.org/ /media/Files/Report\%20Files/2001/Crossing-theQuality-Chasm/Quality\%20Chasm\%202001\%20\%20report\%20brief.pdf. Accessed 26 Nov 2015.

31. Wolf DM, Lehman L, Quinlin R, et al. Effect of patient-centered care on patient satisfaction and quality of care. Journal of Nursing Care Quality 2008;23:316-321.

32. Feo R and Kitson A. Promoting patient-centred fundamental care in acute healthcare systems. International Journal of Nursing Studies 2016;57:1-11.

33. Powers $J$ and Fortney S. Bed baths: much more than a basic nursing task. Nursing. 2014;44:67-68.

34. Berwick DM. A user's manual for the IOM's 'Quality Chasm' report. Health Affairs 2002;21:80-90. 


\section{Chapter 3}

35. Berry LL, Wall EA and Carbone LP. Service clues and customer assessment of the service experience: lessons from marketing. Academy of Management Perspectives 2006;20:43-57.

36. Frow $\mathrm{P}$ and Payne A. A stakeholder perspective of the value proposition concept. European Journal of Marketing 201 1;45:223-240.

37. Moher D, Liberati A, Tetzlaff J, et al. Preferred Reporting Items for Systematic Reviews and Meta-Analyses: The PRISMA Statement. PLoS Medicine 2009;6(7):e1000097. 


\section{Appendix 1 - Search strategies used in PUBMED, MEDLINE, CINAHL and Embase}

\section{Search strategy PUBMED}

Search run 1 as performed at the 25th of February, 2016

(Prepack* OR pack* OR disposable* OR "no rinse" OR "non rinse" OR impregnated OR wet OR "single use" OR care OR "Without water" OR waterless OR basin OR basins OR washbasin* OR washbowl OR alternative OR alternatives OR towel OR bag OR "one step" OR dry)

(in title/abstract)

AND

(wipe OR wipes OR cloth OR cloths OR washcloth* OR glove OR gloves OR mitt OR mitts OR cleanser* OR wash OR washing OR bath OR bathing OR "bed bath" OR "bed wash" OR "body wash")

(in title/abstract)

OR

(Bath MeSH term)

(in all fields)

Filters used:

- $\quad$ Articles published between 1995 - current

- "Clinical trial" or "controlled trial" or "randomized controlled trial"

Search run 2 as performed at the $25^{\text {th }}$ of February, 2016

(Prepack* OR pack* OR disposable* OR "no rinse" OR "non rinse" OR impregnated OR wet OR "single use" OR care OR "Without water" OR waterless OR basin OR basins OR washbasin* OR washbowl OR alternative OR alternatives OR towel OR bag OR "one step" OR dry)

(in title/abstract)

AND

(wipe OR wipes OR cloth OR cloths OR washcloth* OR glove OR gloves OR mitt OR mitts OR cleanser* OR wash OR washing OR bath OR bathing OR "bed bath" OR "bed wash" OR "body wash")

(in title/abstract)

OR

(Bath MeSH term)

(in all fields)

AND

(trial)

(in all fields)

Filters used:

- $\quad$ Articles published between 1995 - current 


\section{Chapter 3}

\section{Search strategy MEDLINE}

Search run 1 as performed at the 25th of February, 2016

(Prepack\$4.ab,ti. OR pack\$4.ab,ti. OR disposable*.ab,ti. OR "no rinse".ab,ti. OR "non rinse".ab,ti. OR impregnated.ab,ti. OR wet.ab,ti. OR "single use".ab,ti. OR care.ab,ti. OR "Without water".ab,ti. OR waterless.ab,ti. OR basin.ab,ti. OR basins.ab,ti. OR washbasin \$1.ab,ti. OR washbowl.ab,ti. OR alternative.ab,ti. OR alternatives.ab,ti. OR towel.ab,ti. OR bag.ab,ti. OR "one step".ab,ti. OR dry.ab,ti.)

AND

(wipe.ab,ti. OR wipes.ab,ti. OR cloth.ab,ti. OR cloths.ab,ti. OR washcloth*.ab,ti. OR glove.ab,ti. OR gloves.ab,ti. OR mitt.ab,ti. OR mitts.ab,ti. OR cleanser*.ab,ti. OR wash.ab,ti. OR washing.ab,ti. OR bath.ab,ti. OR bathing.ab,ti. OR "bed bath".ab,ti. OR "bed wash".ab,ti. OR "body wash".ab,ti.)

OR

(Sub Heading Bath)

(in all fields; "Instrumentation", "Nursing", "Methods" and "Standards" were selected)

Filters used:

- $\quad$ Articles published between 1995 - current

- "Clinical trial" or "controlled trial" or "randomized controlled trial"

Search run 2 as performed at the 25th of February, 2016

(Prepack\$4.ab,ti. OR pack\$4.ab,ti. OR disposable*.ab,ti. OR "no rinse".ab,ti. OR "non rinse".ab,ti. OR impregnated.ab,ti. OR wet.ab,ti. OR "single use".ab,ti. OR care.ab,ti. OR "Without water".ab,ti. OR waterless.ab,ti. OR basin.ab,ti. OR basins.ab,ti. OR washbasin\$1.ab,ti. OR washbowl.ab,ti. OR alternative.ab,ti. OR alternatives.ab,ti. OR towel.ab,ti. OR bag.ab,ti. OR "one step".ab,ti. OR dry.ab,ti.)

AND

(wipe.ab,ti. OR wipes.ab,ti. OR cloth.ab,ti. OR cloths.ab,ti. OR washcloth*.ab,ti. OR glove.ab,ti. OR gloves.ab,ti. OR mitt.ab,ti. OR mitts.ab,ti. OR cleanser*.ab,ti. OR wash.ab,ti. OR washing.ab,ti. OR bath.ab,ti. OR bathing.ab,ti. OR "bed bath".ab,ti. OR "bed wash".ab,ti. OR "body wash".ab,ti.)

OR

(Sub Heading Bath)

(in all fields; "Instrumentation", "Nursing", "Methods" and "Standards" were selected)

AND

(trial.af.)

Filters used:

- $\quad$ Articles published between 1995 - current 


\section{Search strategy CINAHL}

Search run 1 as performed at the $25^{\text {th }}$ of February, 2016

(Prepack* OR pack* OR disposable* OR "no rinse" OR "non rinse" OR impregnated OR wet OR "single use" OR care OR "Without water" OR waterless OR basin OR basins OR washbasin* OR washbowl OR alternative OR alternatives OR towel OR bag OR "one step" OR dry)

(in abstract)

AND

(wipe OR wipes OR cloth OR cloths OR washcloth* OR glove OR gloves OR mitt OR mitts OR cleanser* OR wash OR washing OR bath OR bathing OR "bed bath" OR "bed wash" OR "body wash")

(in abstract)

OR

(Bath MeSH term)

(in all fields; "Bathing" and "Baths" were selected)

Filters used:

- $\quad$ Articles published between 1995 - current

- "Clinical trial" or "randomized controlled trial"

- $\quad$ Apply related words

Search run 2 as performed at the 25th of February, 2016

(Prepack* OR pack* OR disposable* OR "no rinse" OR "non rinse" OR impregnated OR wet OR "single use" OR care OR "Without water" OR waterless OR basin OR basins OR washbasin* OR washbowl OR alternative OR alternatives OR towel OR bag OR "one step" OR dry)

(in abstract)

AND

(wipe OR wipes OR cloth OR cloths OR washcloth* OR glove OR gloves OR mitt OR mitts OR cleanser* OR wash OR washing OR bath OR bathing OR "bed bath" OR "bed wash" OR "body wash")

(in abstract)

OR

(Sub Heading Bath)

(all fields; "Instrumentation", "Nursing", "Methods" and "Standards" were selected) AND

(trial)

(in all fields)

Filters used:

- $\quad$ Articles published between 1995 - current

- $\quad$ Apply related words 


\section{Chapter 3}

\section{Search strategy Embase}

Search run 1 as performed at the 25th of February, 2016

(Prepack\$4.ab,ti. OR pack\$4.ab,ti. OR disposable*.ab,ti. OR "no rinse".ab,ti. OR "non rinse".ab,ti. OR impregnated.ab,ti. OR wet.ab,ti. OR "single use".ab,ti. OR care.ab,ti. OR "Without water".ab,ti. OR waterless.ab,ti. OR basin.ab,ti. OR basins.ab,ti. OR basinless.ab,ti. OR washbasin\$1.ab,ti. OR washbowl.ab,ti. OR alternative.ab,ti. OR alternatives.ab,ti. OR towel.ab,ti. OR bag.ab,ti. OR "one step".ab,ti. OR dry.ab,ti.) AND

(wipe.ab,ti. OR wipes.ab,ti. OR cloth.ab,ti. OR cloths.ab,ti. OR washcloth*.ab,ti. OR glove.ab,ti. OR gloves.ab,ti. OR mitt.ab,ti. OR mitts.ab,ti. OR cleanser*.ab,ti. OR wash.ab,ti. OR washing.ab,ti. OR bath.ab,ti. OR bathing.ab,ti. OR "bed bath".ab,ti. OR "bed wash".ab,ti. OR "body wash".ab,ti.)

Filters used:

- $\quad$ Articles published between 1995 - current

- $\quad$ All clinical trials

Search run 2 as performed at the 25th of February, 2016

(Prepack\$4.ab,ti. OR pack\$4.ab,ti. OR disposable*.ab,ti. OR "no rinse".ab,ti. OR "non rinse".ab,ti. OR impregnated.ab,ti. OR wet.ab,ti. OR "single use".ab,ti. OR care.ab,ti. OR "Without water".ab,ti. OR waterless.ab,ti. OR basin.ab,ti. OR basins.ab,ti. OR basinless.ab,ti. OR washbasin\$1.ab,ti. OR washbowl.ab,ti. OR alternative.ab,ti. OR alternatives.ab,ti. OR towel.ab,ti. OR bag.ab,ti. OR "one step".ab,ti. OR dry.ab,ti.) AND

(wipe.ab,ti. OR wipes.ab,ti. OR cloth.ab,ti. OR cloths.ab,ti. OR washcloth*.ab,ti. OR glove.ab,ti. OR gloves.ab,ti. OR mitt.ab,ti. OR mitts.ab,ti. OR cleanser*.ab,ti. OR wash.ab,ti. OR washing.ab,ti. OR bath.ab,ti. OR bathing.ab,ti. OR "bed bath".ab,ti. OR "bed wash".ab,ti. OR "body wash".ab,ti.)

AND

(trial.af.)

Filters used:

- $\quad$ Articles published between 1995 - current 
chapter

\section{4}

\section{THE EFFECTS OF WASHING WITHOUT WATER VERSUS THE TRADITIONAL BED BATH WITH WATER AND SOAP ON COMFORT AND PHYSICAL DEMANDS: PROTOCOL OF A CROSSOVER RANDOMIZED TRIAL}

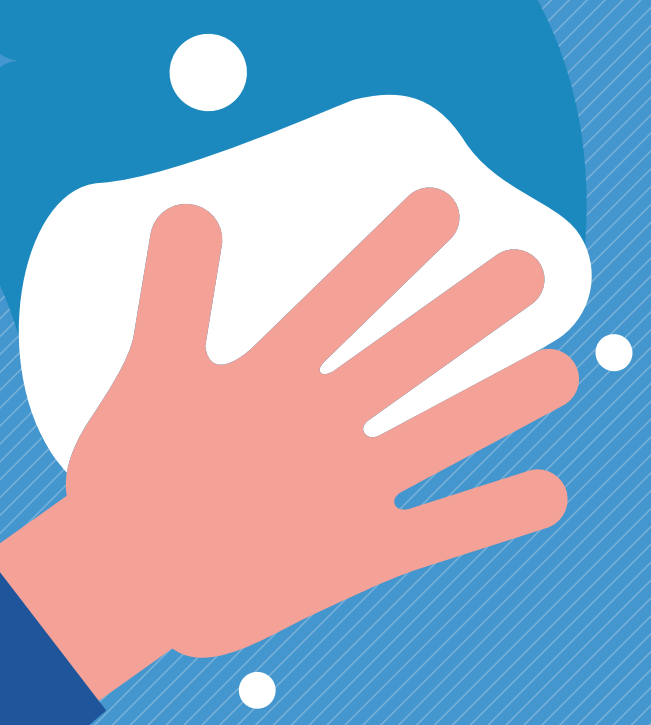

This chapter is available online: www. researchsquare.com/article/rs-7409/vi 


\section{ABSTRACT}

Background: The traditional bed bath with water and soap often leads to emotional and physical discomfort among bedridden patients. In addition, the traditional bed bath is physically demanding for nurses. The washing without water concept is increasingly used in healthcare to bathe bedridden patients and is likely to affect emotional and physical comfort dimensions. This protocol describes a study that will assess how washing without water performs compared to the traditional bed bath regarding emotional and physical comfort experienced by the bathed bedridden person and regarding physical demands for the provider of the bed bath.

Methods: This study uses a randomized crossover design in which nursing students will simulate the bed baths of patients in a laboratory setting. Students will be randomly allocated to a patient or a nurse group and consequently will respectively receive or provide two bed baths: the traditional bed bath and a washing without water bed bath. The order of the bed baths will be randomly determined. Students will receive instructions about the steps of the bathing methods and case descriptions about their role as a patient or nurse. Questionnaires will be used before and after each bed bath to collect data for the primary outcomes of experienced emotional and physical comfort (among students receiving the bed bath) and physical demands (among students providing the bed bath); and, for secondary outcomes related to bed bathing preferences, grades given to the bathing methods and perceived importance of the bed bath. Differences between the two bathing methods will be analyzed, taking the order of the bed baths into account.

Discussion: This study will add to the scientific knowledge of the washing without water bed bath, for which evidence on comfort is currently lacking. If differences in comfort between washing without water and the traditional bed bath are detected among nursing students, it is likely these differences will also be present among actual patients and nurses, as generally, patients are more vulnerable and nurses spend more time bathing patients compared to students.

Trial registration: The trial has been registered in: www.trialregister.nl (TC $=6972$ ) on February 2, 2018. 


\section{BACKGROUND}

Hygiene is described to be one of the essential elements of care that nurses need to provide to persons who cannot bathe independently anymore. Whereas bathing is a self-activity that ensures health and well-being for independent people, bathing often leads to physical and emotional discomfort among persons who cannot bathe independently anymore and, hence, need assistance in bathing. 1, 2 Emotionally, assisted bathing can contribute to comfort but often is experienced by patients as an intrusive encounter. ${ }^{3}$

Bedridden patients require assistance in bathing most often. The bed bath has been defined as "an episode of nursing care where ... patient comfort is provided" (p. 201).4 However, the limited evidence on bed bathing shows that bedridden patients often perceive the bed bath to have a detrimental effect on their level of physical and emotional comfort. The traditional bed bath with water and soap has been perceived as embarrassing, dependent, uncomfortable, and difficult among others. ${ }^{5}$ Moreover, the bed bath with water and soap is physically demanding for both patients and nurses. Nevertheless, most bedridden patients are still bathed with water and soap.

The washing without water concept is increasingly used in healthcare practice as an alternative for the traditional bed bath with water and soap. The concept can be described as disposable, prepacked products for the full body wash consisting of a nonwoven carrier (washcloth) and a no-rinse cleansing fluid that allows nursing staff to bathe someone without the use of water. ${ }^{6}$ Results of a systematic review showed washing without water is a worthy alternative for the traditional bed bath with water and soap. However, the number of studies on washing without water is limited and evidence for patient-related variables, such as physical or emotional comfort is lacking. ${ }^{7}$

Nevertheless, it is very likely that washing without water affects the comfort of patients. ${ }^{8}$ Washing without water can contribute or jeopardize experienced patient comfort directly, both physically and emotionally. Physical comfort pertains to bodily sensations, which in case of a bed bath will probably be influenced by the bathing method applied. ${ }^{9}$ Furthermore, washing without water is expected to impact the emotional comfort of patients, which is defined as "pleasant positive feelings, a state of relaxation that affected the physical status of the body" (p.809). ${ }^{10}$ In one study, the majority of patients indicated they would permanently replace water and soap with washing without water. ${ }^{6}$ It is possible that the preferences of patients are explained by higher levels of perceived comfort, as nurses reported a preference for washing without water when asked about patient comfort in another study. ${ }^{11}$

Washing without water can also make a substantial contribution to experiences of nurses, as bathing assistance will remain a highly prevalent nursing activity.' Evidence shows that the duration of the bed bath with washing without water is shorter compared to the traditional bed bath with water and soap. The reduced time consumption as well as the convenience of the concept have been reported to be reasons for nurses to prefer washing without water to the traditional 


\section{Chapter 4}

bed bath.7 In addition, washing without water is argued to be less physically burdensome for nurses. However, more evidence on physical demands for nurses is required, as this is only studied in unpublished studies. ${ }^{12,13}$

The objective of this study is to assess how washing without water performs compared to the traditional bed bath, with respect to experienced emotional and physical comfort dimensions. Based on previous studies, the research hypothesis is that washing without water is experienced to be 1) equally or more emotionally and physically comfortable by the bathed bedridden person and 2) equally or less physically demanding by the provider of the bed bath. Our study will not only add to the evidence on washing without water but also contribute to the essential care concept (often referred to as fundamental or basic care). Bed bathing belongs to "personal cleansing and dressing," which is one of the physical needs addressed by essential care. ${ }^{14} \mathrm{Feo}$ and Kitson argue that nurses generally undervalue essential care; therefore, bathing is often delivered below par. ${ }^{2}$ Our findings regarding washing without water on comfort can contribute to better bed bathing experiences and, in turn, to the revaluation of bathing as part of essential care.

\section{METHODS}

\section{Study design and participants}

This study uses a randomized crossover design in which the bed baths of patients are simulated in a laboratory setting. Participants (nursing students) will be randomly allocated to a patient or a nurse group and consequently will respectively receive or provide both the intervention, being a bed bath with a washing without water product, and the control bed bath with water and soap. The order of the bed baths will be randomly determined. From now on, students assigned to the patient or the nurse group are called "student patients" and "student nurses," respectively. All student patients and student nurses together are referred to as "participants."

Participants will be recruited out of nursing students of several Dutch intermediate and higher vocational educational institutions in a time span of 2 to 3 years. The study will be executed at the educational institutions in rooms equipped as nursing rooms containing patient beds, bed trolleys and bed curtains. Data will be collected immediately before and after each bed bath. The data will be mainly quantitative, but some qualitative data will also be collected, which will be used to interpret the findings of this study. This study and protocol are based on the "Standard Protocol Items: Recommendations for Interventional Trials" (SPIRIT) statement. ${ }^{15}$ Finally, ethical approval has been obtained by a Dutch medical committee (17-N-172), and the trial is registered at www.trialregister.nl (6972).

Inclusion and exclusion criteria

The study sample will consist of nursing students representing bedridden patients and nurses. All first and second year nursing students are eligible. To be included in the 
study, students should be: 1) willing to bathe fellow students and 2) willing to receive a bed bath in swimming or underwear (allowed to respect the privacy of students) by fellow students. Only first and second year nursing students are included, because these students are likely to lack much practical experience with bed bathing and, therefore, have to attend practice lessons in bathing. No specific exclusion criteria have been determined.

\section{Recruitment and randomization}

Participants will be recruited in close collaboration with the coordinators of first and second year nursing studies at the particular educational institutions. To increase the participation rate, this study will be combined with practical bathing lessons, which are part of the curricula of first and second year nursing studies in which all students will also be instructed about the different bed bathing methods. Furthermore, in lectures all first and second year nursing students will be informed about the trial after which students have the opportunity to ask questions. Students will then receive an information letter accompanied by an informed consent form and an eligibility questionnaire to assess whether students meet the inclusion criteria. Students can hand in the signed form of consent and the completed eligibility questionnaire immediately or within one week after the lecture. Finally, a vlog about the research will be shared with all first and second year nursing students a few days after the lectures to increase participation. Acceptance or refusal to participate will not affect students' study results in any way.

The first author will assess if students who gave informed consent meet the inclusion criteria. Subsequently, unique identifier numbers will be assigned to all eligible students to ensure confidentiality. The identifier numbers will be used to assign participants to the patient or the nurse group, to determine the order of bed baths, and to assign a room and timeslot to participants by using a computer-generated randomization table. Half of the participants receive or provide the washing without water bed bath (intervention) first, whereas the other half starts with the bed bath with water and soap (control). A 1:1 ratio will be used to ensure an equal amount of student patients and student nurses within each group, as every student patient needs a student nurse to provide the bed baths. To keep other factors than the bed bath method as constant as possible, each student patient will receive both bed baths from the same student nurse, at the same time of the day, and in the same bed and room (at each educational institution, between 14 and 20 beds will be available spread across four to six rooms equipped as a nursing room). A wash-out period of one week will be included between the two bed baths.

Before the start of the trial, participants will electronically receive information about the time and place of the bed baths. Furthermore, participants will receive their unique identification number, which they need to write down in every questionnaire used for data collection in this study. Finally, participants will be informed about the role assigned to them (patient or nurse) and the bed bath order (the washing without water bed bath or the traditional bed bath first) before the start of the trial. Figure 1 


\section{Chapter 4}

shows the participant timeline based on the "Consolidated Standards of Reporting Trials" (CONSORT) flow diagram, ${ }^{16}$ including the bed bath order for the different groups of student patients and nurses.

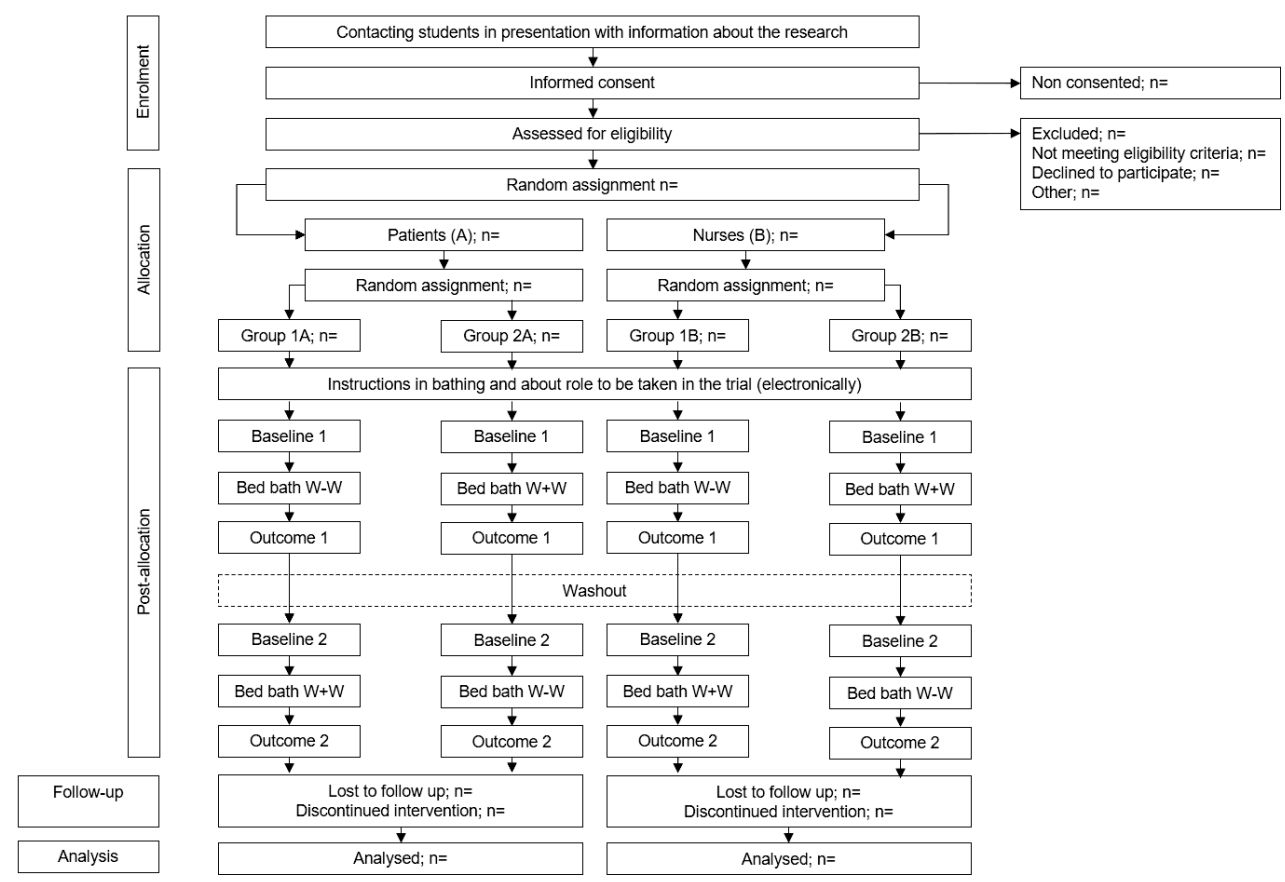

Figure 1 - Study timeline

Legend to Figure 1: " $W-W$ " is a washing without water bed bath and " $W+W$ " stands for the traditional bed bath with water and soap. Groups 1A and 2A (student patients) and 1B and 2B (student nurses) each differ with respect to the order in which the two bed bathing methods take place. In groups $1 \mathrm{~A}$ and 1B, students start with the washing without water bed bath, whereas groups $2 A$ and $2 B$ start with the bed bath with water and soap.

\section{Description of intervention and control}

The intervention in this study is a washing without water bed bath that uses a disposable, prepacked product for the full body wash consisting of eight nonwoven gloves with a no-rinse, fragranced cleansing fluid. ${ }^{6}$ The traditional bed bath approach with washbasins, water, soap, towels, and washing mitts will be used in the control group, as this is still the most adopted bed bathing method in healthcare.

To ensure uniform execution of the bed baths, participants will receive verbal and written step-by-step instructions for both bed bathing methods (instructions are available upon request). Apart from the methods used, the instructions of the washing without water bed bath and the traditional bed bath are highly similar. The instructions are aligned with the educational institutions and developed for this study based on bathing protocols obtained from the Internet (for the control bed bath) ${ }^{17}$ and from a 
washing without water supplier (for the intervention bed bath). Research assistants will observe the bed baths for compliance with the instructions.

In addition to the bathing instructions, participants will obtain instructions related to the role assigned to them. Student patients will receive a case description, which describes the case of a patient who broke his or her right leg. Hence, instructions are provided about which consequences this injury has for the steps during the bed bath (e.g. they can wash their face themselves but need help in sitting up). Student nurses are instructed to confine communication during the bed bath and will be provided with a case description that explains to them that the patient they are about to bathe is the second of many patients that need to be bathed in a limited period. All student patients and all student nurses will receive the same case descriptions without any variation. Although case descriptions cannot mimic reality completely, these instructions at least to some extent simulate the situations real patients and nurses encounter. ${ }^{18}$

\section{Data collection and measures}

Data will be collected by paper questionnaires that all participants need to fill out right before and immediately after each bed bath. Hence, for each bed bath, we have two baseline (B) questionnaires (one for student patients and one for student nurses) that need to be filled out before the bed bath, and two outcome (O) questionnaires that need to be filled out after the bed bath; again, one for each group of participants. Table 1 shows which outcomes are included in the questionnaires before each bed bath (B1 and B2 in Table 1 for the questionnaire before the first and the second bed bath, respectively) and in the questionnaires after each bed bath (O1 and 02) for each of the participant groups. The group of student patients and student nurses will fill out the questionnaires in separate rooms. Participants have 30 minutes for one bed bath, excluding the completion of the baseline and outcome questionnaires, which will take approximately 15 minutes.

\section{Demographic data}

Demographic data of all participants will be collected at baseline (B1) to describe the study sample, including age, weight, length, gender, educational level, and experience with bed baths obtained before the trial. 


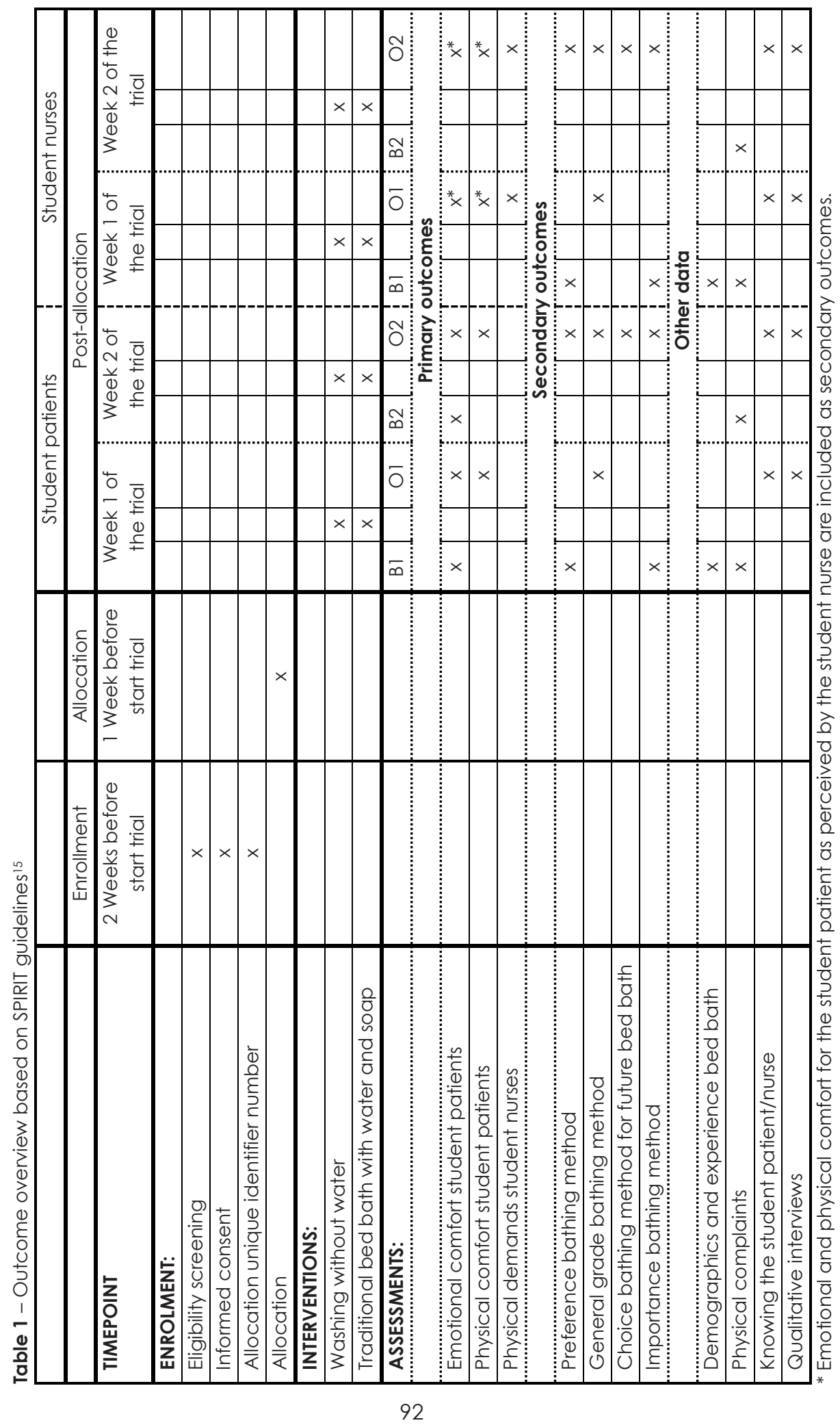




\section{Primary outcome data}

The primary outcomes related to student patients are experienced emotional and physical comfort, which will be measured using validated scales. To measure emotional comfort, the 12-item Patient Evaluation of Emotional Comfort Experienced (PEECE) scale will be used. ${ }^{19}$ Student patients need to indicate to what extent they experienced certain feelings during the bed bath (relaxed, valued, safe, calm, cared for, at ease, like smiling, energized, content, in control, informed, thankful) on a fivepoint scale. The cumulative score will be used in the analysis, ranging from 12 to 60; a high score meaning a high level of experienced emotional comfort. High Cronbach a coefficients (between 0.74 and 0.88 ) have been reported for the PEECE scale in previous studies. ${ }^{19}$ Physical comfort will be measured by a single item question asking the student patient about the perceived comfort of the bed bath on a ten-point Likert scale ranging from "very uncomfortable" to "very comfortable".

The physical demands scale will be used to measure physical demands imposed by the bed bath on student nurses. ${ }^{20}$ The scale consists of seven items related to physically demanding activities (e.g. carrying or moving weights heavier than 10 kilograms) or postures (e.g. standing for a long time). Student nurses have to indicate to what extent these activities and postures were present during the bed bath on a five-point Likert scale. The cumulative score will be used for the data analysis, ranging from seven to 35; a high score meaning high physical demands. Previous studies that used the physical demands scale reported high Cronbach a coefficients of 0.87 and 0.91 , respectively. ${ }^{20,21}$

\section{Secondary outcome data}

In addition to the primary outcomes, several measures are related to the secondary outcomes of this trial:

- To assess emotional comfort for student patients perceived by student nurses, the PEECE scale is also filled out by student nurses.

- Physical comfort for student patients as perceived by student nurses is measured by a single item question similar to the question about physical comfort asked to student patients directly.

- The bed bathing method preference among student patients will be measured in several ways. Student patients will be asked directly which bathing method they prefer after they received both the intervention and control bed bath. Furthermore, after each bed bath, student patients are asked to give an overall grade to the bathing method they just received, ranging from one (very bad) to ten (very good). Finally, after both bed baths have been received, student patients are asked how they would like to be bathed in the future.

- In addition, student nurses will be asked directly for their bathing method preference and for an overall grade for both bathing methods. Finally, after both bed baths have been provided, student nurses are asked which type of bed bath they would like to provide in the future. 


\section{Chapter 4}

- The perceived importance of the bed bath according to all participants (patient and student nurses) will be measured by using a numeric rating scale from one (being not important at all) to 10 (being extremely important).

- $\quad$ The duration of the bed bath will be calculated using the start and end time of each bed bath as recorded by the research assistants observing the bed baths.

Process evaluation

In addition to the primary and secondary outcomes, the process of some of the bed baths provided during the trial will be evaluated by research assistants with a background in nursing who will mainly focus on fidelity (if the bed baths were conducted as planned). Furthermore, short interviews with a sample of participants will be held to gather experiences with both bathing methods and to assess the satisfaction of participants with the trial. The qualitative data will be used in the interpretation of the outcome findings.

\section{Participant retention}

The trial will be combined with practice lessons in bathing, which are part of the regular curriculum of first and second year nursing students. Furthermore, participants will fill out the questionnaires right before and immediately after each bed bath. Hence, measures to promote participant retention and to complete follow-up are not necessary. However, it is likely participants will drop out due to the wash-out period and because they may choose to withdraw from the trial. To increase the chance of complete follow-up, participants will frequently be informed and reminded of the study through lectures and information via email or on the electronic learning environment with support of the educational institutions.

\section{Reliability}

The laboratory setting offers opportunities to increase fidelity among participants. Participants will receive elaborate instructions including a step-by-step description of the intervention and the control bed bath. In addition, participants will only have the bathing materials at their disposal that are needed for the particular bed bath they are expected to receive or provide. Hence, intention to treat analyses will not be needed for this study.

Reliability will also be taken into account in the data management. Referential data rules, valid values, and range checks will be performed to check the data. Furthermore, the data will be checked for outliers, and a second researcher will inspect a subset of the data to detect possible errors.

\section{Power and sample size}

A power calculation for paired measurements shows that in total at least 104 participants should be recruited; 22 half of them being student patients, half of them being student nurses. The sample size is based on the primary outcome of emotional 
comfort, with an effect size of 0.5 , a type I error of 0.05 and $95 \%$ power. First and second year nursing students (at least 700 in total) of the participating educational institutions will be contacted to participate in the trial, which is expected to be sufficient given the assumed attrition rate of $15 \%$.

\section{Data analysis}

In case we do not have missing values, a one sample t-test will be executed to test for the significance of the change in scores between the two bathing methods with respect to experienced emotional and physical comfort, physical demands, overall grades, and bed bath duration. Additionally, a one sample t-test will be performed to test for differences in perceived importance of the bed bath before and after the trial. Instead, a two-level regression analysis will be applied in the case of missing values, ${ }^{23}$ with repeated measurements on the first level and participants on the second level. Under the assumption of missing completely at random, a two-level regression analysis offers an unbiased and more efficient method compared to the one sample t-test on change in scores. ${ }^{24}$ Preferences for bed bath methods will be tested by using a simple binomial test.

Because of the crossover design, additional analyses will be executed to test whether the dependent outcomes (experienced emotional and physical comfort, physical demands, overall grades, bed bath duration, and importance of the bed bath) differ depending on the order of the bed baths. Hence, the order of the bed bath will be included as a dummy variable in separate regression analyses together with time and an interaction variable between time and order of the bed bath. Finally, the influence of the bed bath order on preferences for bathing methods will be tested using the direct likelihood method.

Possibly, some variables will be included as covariate in the regressions if enough variation on the particular variables is found within the study sample. Potential covariates for which data will be collected during the trial are:

- Educational level of the participating nursing students (possible covariate of all outcomes);

- Emotional comfort experienced by the student patient before the bed bath (possible covariate of experienced emotional and physical comfort of the bed bath);

- Performance assessment by the student patient of how the student nurse executed the bed bath (possible covariate of experienced emotional and physical comfort of the bed bath and the grade given to the bed bath);

- Self-assessment by the student nurse (possible covariate of emotional and physical comfort of the bed bath as perceived by the student nurse);

- Physical complaints before the bed baths among all participants (possible covariates of experienced physical comfort of the bed bath and physical demands of the bed bath);

- Knowing or not knowing the patient or the student nurse (possible covariate of experienced emotional comfort). 


\section{Chapter 4}

Finally, additional analyses will be conducted to detect possible differences in baseline measurements between the different groups (student nurses and patients, participants starting with the intervention or the control bed bath, participants who completed the trial and dropouts, and nursing students from different educational institutions). All randomized participants will be included in the main analyses, regardless of protocol adherence. IBM® SPSS ${ }^{\circledR}$ Statistics, version 25 will be used for the data analysis. Confidence intervals of 0.05 will be applied, and the results will be presented with a $5 \%$ threshold.

\section{Data monitoring and management}

The data will be stored on a central and secured directory, which is only accessible by the research team for at least 10 years after last publication. Furthermore, as the data collection will only take two days for each group of students (excluding the wash-out period) and the risks for participants during the trial are basically nonexistent, there is no need for periodic inspection of accumulating data to make adjustments accordingly.

\section{DISCUSSION}

The objective of this study is to measure the effects of the washing without water bed bath on experienced emotional and physical comfort of persons being bathed and on physical demands of the washing without water bed bath among persons providing the bed bath. Despite that the bed bath in general has been reported often to have a detrimental impact on comfort, the experiences of patients and nurses with washing without water regarding comfort have not been thoroughly assessed. Therefore, this study's results offer a valuable contribution to the scientific knowledge on the washing without water bed bath. We think this knowledge will increase the awareness among healthcare providers of the impact of the bed bath on patients and consequently will increase the perceived importance of this essential nursing care activity.

This study's crossover design, reduces the risk of bias, allows a smaller sample size, and gives participants the possibility to experience both types of bed baths, either as a patient or as a nurse. ${ }^{25}$ Moreover, the crossover design is most suitable to measure preferences for bathing methods, which is included as a secondary outcome, because participants will experience both types of bed baths. ${ }^{26}$ We want to comment on two often underreported methodological issues related to crossover trials, which are allocation concealment and carryover effects. ${ }^{26}$ In this study, the allocation of participants to the different groups will not be concealed, as blinding of participants to the different bed bathing methods is impossible. Furthermore, carryover effects are unlikely, because the effects of the bed baths are short-lived and because of the one week wash-out period.

A laboratory setting was chosen to be able to measure causation between the bed bath and comfort by isolating the bed bath from other nursing activities that 
might influence comfort as well. It would be very difficult to assess the effects of the bed bathing method on comfort in a natural setting, in which the bed bath method is only one of many uncontrollable nursing elements. ${ }^{27}$ Our laboratory experimental design enables a high internal validity because of the control of possible confounding variables, such as time of the bed bath and the person providing the bed bath, which are held constant in our study. 25

As part of the laboratory setting, students will be recruited for several reasons. First of all, it would be burdensome for actual bedridden persons to participate in this study, especially given the laboratory setting. Second, the use of students offers advantages not only to the research team (e.g. expected relative ease of access to potential participants) but also enables students to obtain personal experience in bathing methods. Hence, participating students will obtain valuable knowledge for their future careers, which in turn can have positive spillover effects for the patients who will be bathed by these students in the future.

We are convinced that the strengths of the laboratory setting with respect to internal validity outweigh its often stated weakness of artificiality. ${ }^{27}$ One can argue that the use of students adds to the artificiality of our study because actual patients are generally more vulnerable and nurses spend more time bathing compared to students. However, especially because of these differences between students and actual patients and nurses, it is likely that the differences found between washing without water and the traditional bed bath on comfort among students will also be found among actual patients and nurses. That is, the impact of the bed bath is probably higher for actual patients and nurses compared to students. Nevertheless, although students differ in many ways from actual patients, they are likely to encounter similar feelings of dependency and tension during the bed bath. 


\section{References}

1. Rader J, Barrick AL, Hoeffer B, et al. The bathing of older adults with dementia: easing the unnecessarily unpleasant aspects of assisted bathing. American Journal of Nursing 2006;106:40-48.

2. Feo R and Kitson A. Promoting patient-centred fundamental care in acute healthcare systems. International Journal of Nursing Studies 2016;57:1-11.

3. Downey $\mathrm{L}$ and Lloyd $\mathrm{H}$. Bed bathing patients in hospital. Nursing Standard 2008;22:35-40.

4. Coyer FM, O'Sullivan J and Cadman N. The provision of patient personal hygiene in the intensive care unit: a descriptive exploratory study of bed-bathing practice. Australian Critical Care $2011 ; 24: 198-209$.

5. Lopes JL, Nogueira-Martins LA and de Barros AL. Bed and shower baths: comparing the perceptions of patients with acute myocardial infarction. Journal of Clinical Nursing 2012;22:733-740.

6. Schoonhoven L, van Gaal BG, Teerenstra S, et al. Cost-consequence analysis of "washing without water" for nursing home residents: a cluster randomized trial. International Journal of Nursing Studies 2015;52:112-120.

7. Groven FM, Zwakhalen SM, Odekerken-Schröder G, et al. How does washing without water perform compared to the traditional bed bath: a systematic review. BMC Geriatrics 2017;17:1-16.

8. Tutton E and Seers K. Comfort on a ward for older people. Journal of Advanced Nursing 2004;46:380-389.

9. Kolcaba KY. A theory of holistic comfort for nursing. Journal of Advanced Nursing 1994;19:1178-1184.

10. Williams AM and Irurita VF. Therapeutic and non-therapeutic interpersonal interactions: the patient's perspective. Journal of Clinical Nursing 2004;13:806-815.

11. Larson EL, Ciliberti T, Chantler C, et al. Comparison of traditional and disposable bed baths in critically ill patients. American Journal of Critical Care 2004;13:235241.

12. Knibbe H, Geuze $L$ and Knibbe N. Ergonomische aspecten van wassen-zonderwater voor zorgverleners. $2005 . \quad$ Available from: http://docs.minszw.nl/pdf/92/2005/92_2005_1_9249.pdf. Accessed 13 Aug 2015.

13. Knibbe JJ and Knibbe NE. Businesscase verzorgend wassen wassen-zonder-water. Stichting RegioPlus/Platform Zorginnovatie, Zoetermeer. 2006. Available from: http://www.locomotion.nu/doc/innovatiespiegel/BusinesscaseVW.pdf. Accessed 13 Aug 2015.

14. Feo R, Conroy $T$, Jangland $E$, et al. Towards a standardised definition for fundamental care: a modified Delphi study. Journal of Clinical Nursing 2018;27:2285-2299.

15. Chan A, Tetzlaff JM, Gøtzsche PC, et al. SPIRIT 2013 explanation and elaboration: guidance for protocols of clinical trials. BMJ 2013.

16. Moher D, Hopewell S, Schulz K, et al. CONSORT 2010 explanation and elaboration: updated guidelines for reporting parallel group randomised trials. BMJ 2010;340:128.

17. Protocol "wassen van een zorgvrager op bed". Available from: http://www.factore.nl/helpende/images/stories/Helpende/Beroepstaak_7_Wassen_en_aan_en_vitkleden/Protocollen/Protocollen\%20versie\%202/BT\%207\%20Protocol\%20Wa 
ssen\%20van\%20een\%20zorgvrager\%20op\%20bed\%20v2.pdf. Accessed 1 Feb 2018.

18. Hughes R and Huby M. The application of vignettes in social and nursing research. Journal of Advanced Nursing 2002;37:382-386.

19. Williams AM, Lester L, Bulsara C, et al. Patient Evaluation of Emotional Comfort Experienced (PEECE): developing and testing a measurement instrument. BMJ Open 2017;7.

20. de Jonge J, Mulder MJ and Nijhuis FJ. The incorporation of different demand concepts in the job demand-control model: effects on health care professionals. Social Science \& Medicine 1999;48:1 149-1160.

21. Verbeek H. Redesigning dementia care - An evaluation of small-scale, homelike care environments. 2011 (Doctoral dissertation).

22. Lachin JM. Introduction to sample size determination and power analysis for clinical trials. Control Clinical Trials 1981;2:93-113.

23. Snijders TA and Bosker RJ. Multilevel analysis: an introduction to basic and advanced multilevel modeling. Sage; 1999.

24. Hox JJ. Multilevel analysis: techniques and applications, 2nd ed. New York: Routledge; 2010.

25. Wilson TD, Aronson E and Carlsmith K. The art of laboratory experimentation. In: Fiske ST, Gilbert DT and Lindzey G, editors. Handbook of social psychology. Hoboken: Wiley; 2010:51-81.

26. Mills EJ, Chan AW, Wu P, et al. Design, analysis, and presentation of crossover trials. Trials 2009;10:1-6.

27. Richards DA and Hamers JP. RCTs in complex nursing interventions and laboratory experimental studies. International Journal of Nursing Studies 2009;46:588-592. 

chapter

\section{5 \\ COMFORT DURING THE \\ BED BATH - A RANDOMIZED CROSSOVER TRIAL ON THE EFFECT OF WASHIN G WITHOUT WATER VERSUS WATER AND SOAP IN NURSING STUDENTS}

This chapter was published as:

Groven FM. Zwakhalen SM. odekerken-Schröder G, Tan, F, and Hamers JP. Comfort during the bed bath

- A randomised crossover trial on the effect of washing without water versus water and soap in nursing students. Journal of Clinical Nursing 2021:30:2234-2245 doi.org110.1111/ijoen. 15610 


\section{ABSTRACT}

Aims and objectives: To compare the washing without water method with the water and soap method regarding comfort perceptions of the bed bath.

Background: Bathing affects nurses' and care recipients' comfort. Bedridden care recipients can be bathed in bed with water and soap or with washing without water products. Little is known about the differences between these two bed bath methods regarding comfort perceptions among care recipients and nurses.

Design: Crossover randomized laboratory controlled trial, conducted from March 2018 to November 2019, according to the CONSORT guidelines.

Methods: Nursing students were randomly allocated roles as a patient (who received both types of bed baths) or a nurse (who provided both types of bed baths). Also, the order in which the bed baths were received/provided was randomized. A total of 97 students were included in the analysis. Student patients filled out the Patient Evaluation of Emotional Comfort Experienced (PEECE) scale to measure emotional comfort and a single-item question on physical comfort after each bed bath. Student nurses filled out the Physical Demands scale after each bed bath to measure their physical comfort perceptions.

Results: No differences were found between the two bed bathing methods regarding student patients' emotional or physical comfort levels. Among student nurses, the washing without water method was less physically demanding than the water and soap method.

Conclusions: Taking into account time-efficiency and physical comfort for nurses, washing without water seems to be a valuable alternative to water and soap from a care recipient comfort perspective, which should be assessed in a clinical setting in future research.

Relevance to clinical practice: The washing without water method is less physically demanding for nurses and takes less time. It does not have a detrimental effect on care recipients' emotional and physical comfort.

\section{Acknowledgements}

We would like to express our gratitude to our contacts at the bachelor schools of nursing who participated in this trial, in particular to Marijke van Hommerich-Cronenberg for her enthusiasm, willingness and commitment in setting up the research and recruiting participants. Furthermore, we would like to thank all research assistants who completed the observations of the bed baths. 


\section{INTRODUCTION}

Bathing and showering are important personal care activities that most of us are able to fulfill independently. However, when people age, independent bathing can rapidly become less self-evident. ${ }^{1}$ At least $15 \%$ of Europeans aged 65 or older have severe difficulties with bathing and showering. ${ }^{2}$ Within health care institutions, many care recipients require assistance in bathing from nursing staff; this particularly applies to immobile care recipients who often need to be bathed in bed due to cognitive and physical decline. ${ }^{3}$

Bathing is part of the essentials of care, which embody nursing care activities that care recipients deem most vital for their well-being. ${ }^{4,5}$ As an essential of care activity, bathing has a substantial impact on nurses and care recipients, as became evident in a qualitative study about experiences of the bed bath. ${ }^{6}$ For many nurses, the daily morning activities consist for the most part of bathing care recipients. For care recipients, bathing is often one of the first activities of the day. Moreover, bathing contributes to care recipients' well-being by fulfilling hygiene and comfort needs. ${ }^{7}$

Nurses and nursing students often undervalue the impact of bathing on care recipients and perceive it as a routine nursing activity that does not require much skill. ${ }^{4} 8$ However, care recipients' comfort is compromised if the bed bath is not provided properly. ${ }^{9}$ Comfort is a source of care recipients' well-being and is described as a holistic and multidimensional experience, including physical and emotional dimensions. ${ }^{7}$, 10-12 Physical comfort is related to the relief of physical discomforts, including pain. ${ }^{13}$ Emotional comfort relates to the experience of positive emotions (e.g. feeling valued and at ease) and the absence of negative emotions (e.g. feeling dependent). ${ }^{12}$

One of the few interventions that aims to optimize bed bathing is washing without water. This intervention consists of prepacked products containing disposable nonwoven washcloth with a cleansing fluid that does not have to be rinsed. ${ }^{14}$ Evidence regarding washing without water with respect to comfort for care recipients and nurses is limited. To guide nursing practice, the current study explores differences between the traditional water and soap method and the washing without water method with respect to physical and emotional comfort among people bathed in bed, and physical comfort among people providing the bed bath. Consequently, this study adds to the limited scientific evidence on essentials of care, such as the bed bath. ${ }^{15,} 16$

\section{BACKGROUND}

For both care recipients and nurses, comfort in bathing is important. Because bathing is a substantial part of the morning routine, an uncomfortable bed bath could literarily mean a bad start to the day or shift. Unfortunately, many bedridden people perceive the bed bath to have a detrimental impact on their levels of physical and emotional comfort.3, 17, 18 With respect to emotional comfort, bedridden care recipients can feel embarrassed and frustrated and might feel their body is violated during the bed 


\section{Chapter 5}

bath.16, 19 Consequently, feelings of dependency and a lack of dignity reduces bedridden care recipients' level of emotional comfort.20 Physical comfort is particularly pressured when bedridden care recipients experience pain on movement, feel weak and tired, or are cold.16, 21 For nurses, the bed bath can be physically demanding and a source of physical complaint, which reduces their level of physical comfort. 22,23

The applied bed bath method can have an influence on bedridden care recipients' levels of emotional and physical comfort and on nurses' level of physical comfort. Traditionally, bedridden people are bathed in bed with water and soap. As an alternative, washing without water is increasingly used in health care, particularly in Europe and the US. ${ }^{14,24,25}$ In a systematic review, washing without water has been shown to be at least as effective as water and soap with respect to hygiene, skin condition and bathing completeness. ${ }^{26}$ However, the results of this review also show that the number of studies on washing without water is low, especially with respect to users' emotional and physical comfort perceptions.

Based on extant studies, one could expect that care recipients perceive washing without water to be at least as comfortable as water and soap bed baths. A majority of care recipients have been found to perceive washing without water as a valuable alternative or replacement to the water and soap method. ${ }^{14,27}$ Moreover, care recipients perceive washing without water products as soft, easy to use, fast, and convenient. ${ }^{18,27}$ In addition, most nurses perceive washing without water to be comfortable for care recipients and think that care recipients are satisfied with this type of bed bath. ${ }^{27}, 28$ However, it is unknown how these results relate to care recipients' actual physical and emotional comfort levels.

Also with respect to physical comfort levels among nurses providing the bed bath, not much evidence is given in existing studies. Nurses have been found to perceive the washing without water method as a convenient, easy to administer, and valuable alternative to the water and soap method. ${ }^{14,27-29}$ However, just like much is unknown about care recipients' emotional and physical comfort perceptions, more evidence is also needed on nurses' physical comfort perceptions.

Based on the available evidence, we state the following two hypotheses:

1. The washing without water method is equal to or more physically and emotionally comfortable for care recipients as compared to the water and soap method.

2. The washing without water method is equal to or more physically comfortable (less physically demanding) for nurses as compared to the water and soap method.

\section{Purpose of the study}

The aim of the current study is to compare the washing without water method with the water and soap method with respect to perceived physical and emotional comfort among people bathed in bed and with respect to perceived physical comfort among people providing the bed bath. 


\section{METHODS}

\section{Research design}

A non-blinded crossover laboratory design was used in which participants were randomly allocated to one of two arms, each with a different order of treatments, being the bed bath with washing without water and the bed bath with water and soap. A more detailed overview of the research design is provided in the protocol, which is available online. ${ }^{30}$ The study complies with the guidelines of the Consolidated Standards of Reporting Trials (CONSORT).

\section{Setting and sample}

To have maximum control over the course of the trial (e.g. participants' adherence to the order of the bed baths and the correct use of bed bath materials) and to control for confounders (e.g. people providing the bed bath, communication during the bed bath, and location of the bed bath), a controlled environment was set up in a laboratory setting. ${ }^{31,32}$ The research population consisted of first year nursing students, which was deliberately chosen for practical and theoretical reasons. Practically, it was more feasible for nursing students to participate in our laboratory setting compared to real bedridden care recipients, who would also be difficult to randomly allocate to different bed bath methods. Theoretically, nursing students do not yet have much experience with the bed bath (neither as a care recipient, nor as a nurse) and therefore are less prejudiced about the different bed bath methods. Furthermore, nursing students are likely to be more homogenous compared to real care recipients who, for example, have varying physical and cognitive conditions which cannot be controlled.

Four different groups of first year nursing students (of at least 150 students each) from two bachelor schools of nursing were informed about the research through lectures, posts on the electronic learning environments, vlogs, and personal communication by teachers. In total, approximately 750 students were informed about the research. All first year nursing students were eligible if they consented to bathe or be bathed by fellow students. Because the curriculum of first year nursing students includes bed bathing training and practicing the different types of bed baths contributes to their professional development, all participants obtained study points. Altogether, the four groups of students that consented to participate consisted of 120 nursing students, of which 111 nursing students were enrolled in the study (18 to 40 participants per group).

Nursing students that were enrolled (from now on called participants) were randomly allocated to the role of a patient (from now on called 'student patients') or to the role of a nurse (from now on called 'student nurses') and simulated bed baths in the teaching rooms of their nursing school that were equipped as nursing home rooms. Each student patient was coupled randomly to a student nurse. Furthermore, each couple was randomly assigned to one of the two arms of the trial. For all allocations, randomization was applied with a computer-generated randomization 


\section{Chapter 5}

table containing unique identifier numbers assigned to the participants, which concealed allocation for the first researcher. Each student patient/nurse received/provided one bed bath per day with a wash-out period of one week between the two different bed baths as shown in Figure 1.

To ensure that the bed baths were correctly executed, participants were instructed on both bed bath methods and only had materials at their disposal that were required for the type of bed bath they needed to receive or provide. Furthermore, both student patients and student nurses received a script before the start of the trial that described how to act within their role to standardize the execution of the bed baths and increase the realism of the experiment as much as possible. The script for student patients for example, contained information about their physical condition and the constraints they had to imagine during the bed bath (e.g. that they had broken their leg and therefore could not turn independently). Furthermore, to control for interpersonal dynamics and communication during the bed baths that might affect participants' comfort experiences of the bed baths, both student patients and student nurses were instructed to limit communication during the bed baths. Student nurses were only allowed to explain the different steps of the bed bath and to ask predetermined questions to student patients during the bed bath (e.g. "do you want me to apply deodorant or do you want to do that yourself?"). Student patients were informed about the required answers to student nurses' questions (e.g. that they want to apply deodorant themselves). Although interpersonal dynamics and communication are important in bed baths provided in actual health care practice, these variables are controlled for in our experiment to assess the differences between the two bed bath methods.

\section{Data collection}

Data were collected between March 2018 and November 2019 from the four groups of participants (recruited out of the four different groups of first year nursing students). The number of students that consented to participate was deemed sufficient to reach the required sample size of 104 participants based on a power calculation with an effect size of 0.5 on student patients' emotional comfort, a type 1 error of 0.05 , and $95 \%$ power. Before and after each bed bath, all participants filled out a questionnaire, which contained items and scales related to our primary and secondary outcomes. 


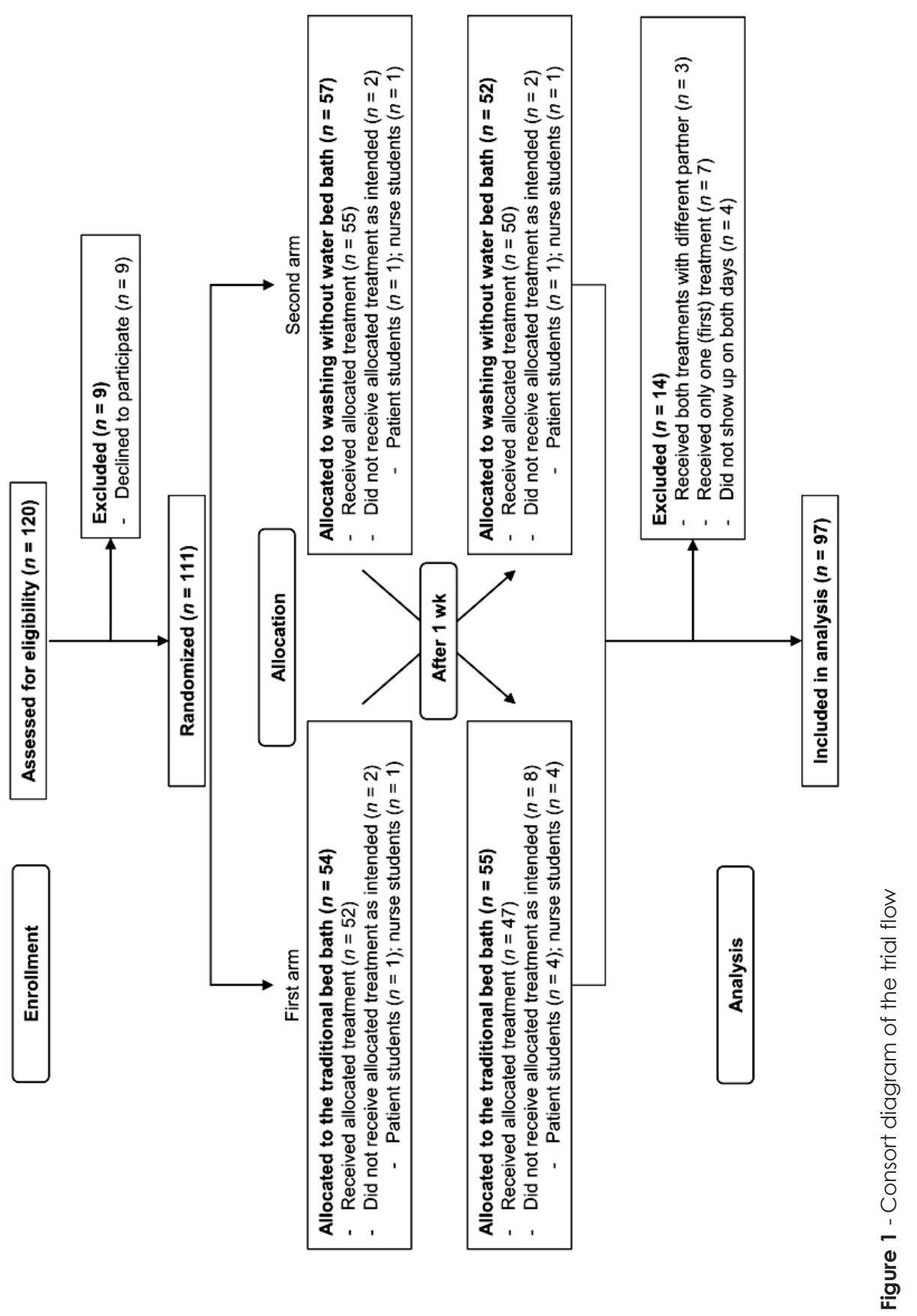




\section{Chapter 5}

\section{Primary outcomes}

Among student patients, the following primary outcomes were measured. First, emotional comfort during the bed bath among student patients was measured using the validated Patient Evaluation of Emotional Comfort Experienced (PEECE) scale for which Cronbach's alpha values between .74 and .88 have been reported. ${ }^{33}$ The PEECE scale consists of 12 items, each related to a positive feeling, which needed to be answered on a 5-point Likert scale, ranging from one (not at all experienced) to five (very much experienced) after each bed bath. The cumulative score (ranging from 12 to 60) was used for the analysis with a high score indicating a high level of emotional comfort during the bed bath. Second, to measure physical comfort among student patients, a single item question about the physical comfort of the bed bath had to be answered on a scale from 1 (very uncomfortable) to 10 (very comfortable) after each bed bath.

Among student nurses, physical comfort was the primary outcome. To assess physical comfort, the validated 7-item Physical Demands scale was completed after each bed bath. Previously reported Cronbach's alpha coefficients between .87 and .91 indicate a high reliability of this scale.34, 35 The items are related to physically demanding activities or postures and can be answered on a scale from 1 (not at all present during the bed bath) to 5 (very much present during the bed bath). The cumulative score (ranging from 7 to 35) was used for the analysis with a low score (low physical demands) indicating a high level of physical comfort during the bed bath.

\section{Secondary outcomes}

Among the secondary outcomes were participants' bed bath preferences after study completion. With respect to bed bath preferences, both student patients and student nurses were asked two questions: 1) which bed bathing method they preferred, and 2) which bed bathing method they would choose for a (hypothetical) future trial. Furthermore, all participants were asked to grade each bed bath method on a scale from 1 (very bad) to 10 (very good). In addition, the duration of the bed baths was measured by the research assistants who observed the bed baths. In addition to the secondary outcomes related to all participants, we asked student patients about the general importance of the bed bath from the patient perspective on a scale from 1 (not at all important) to 10 (very important) both before and after the trial. Also, student nurses were asked the same questions about the general importance of the bed bath, but from the nurse perspective. Furthermore, student nurses were asked to fill out the PEECE scale and the single-item physical comfort question on behalf of the student patient after each bed bath to measure student nurses' perceptions of student patients' emotional and physical comfort during the bed bath.

\section{Data analysis}

Statistical analysis was performed in IBM® SPSS $®$ Statistics, version 25. Participants were excluded from the analysis if they did not undergo both bed baths and therefore missed one element of data collection. Two participants who were included in the 
analysis, did not fill out all items of the scales measuring emotional comfort, for which the mean values of the total group of participants for the particular items were imputed. ${ }^{36}$ Afterwards, differences in baseline characteristics were assessed between the four groups of participants with respect to gender, age, length, weight, experience with the bed bath, bed bath method preferences, and perceptions about the importance of the bed bath. Due to the limited amount of information about scale reliability in existing literature, reliability was assessed for the PEECE and the Physical Demands scales by calculating Cronbach's alpha coefficients, which are reported in appendix 1.

To detect possible differences between the two bed bath methods regarding our primary comfort outcomes, paired sample t-tests were executed. Paired sample t-tests were also performed for the secondary outcomes: grades assigned to the two bathing methods, duration of the bed bath, and emotional and physical comfort of the bed bath for student patients according to student nurses. Also, differences in perceived importance of the bed bath among student patients and student nurses before and after the trial were assessed by means of a paired sample t-test. Bed bath method preferences were assessed by chi-square goodness-of-fit tests, testing equal counts for the water and soap bed bath, washing without water, or having no preference. Finally, additional linear mixed regression models were conducted in which the first level included the repeated measures and the second level the participants. These analyses allowed us to assess the effects of potential covariates on our continuous outcome variables. In a separate linear mixed regression, an interaction variable "method*order" was included (i.e. method represents the type of bed bath received/provided, and order, whether the type of bed bath was received/provided first or second in the trial) to assess whether the effect of the bed bath method on our outcome variables depended on the order in which the two types of bed baths were received/provided.

Numbers and percentages are presented for categorical data. For continuous variables, means and standard deviations (SD) are presented. For all outcomes, $p<.05$ is considered as statistically significant. For every statistically significant result from the paired samples t-test, the effect sizes have been determined by calculating Cohen's $d$ using an online effect size calculator. Based on Cohen, the effect size is considered to be small, medium, or large at values equaling or exceeding $.2, .5$, and .8 respectively. ${ }^{37}$ Finally, $95 \%$ confidence intervals are reported.

\section{Ethical approval}

This study conforms to the Declaration of Helsinki, has been evaluated and waived for approval by a Dutch Medical Ethics Committee, and is registered at www.trialregister.nl (ID = NL6787). Participants provided written consent before the start of the study. 


\section{Chapter 5}

\section{RESULTS}

\section{Sample characteristics}

Out of four different groups of first year nursing students, in total 120 nursing students gave initial consent. The four groups of participants were highly similar. Although in one of the groups, students had relatively more experience with providing a bed bath and had a relatively high preference for the washing without water method prior to the trial, these small differences did not affect the results. Out of the 120 participants, nine declined to participate before commencement of the trial. Therefore, eventually 111 nursing students were randomly allocated of which 14 participants were excluded from the analysis because they did not complete both of the bed baths that were part of the crossover trial. In total, 97 students completed both bed baths as intended and were included in the analysis (Figure 1).

Table 1 - Sample characteristics

\begin{tabular}{|c|c|c|c|}
\hline Variables & $\begin{array}{l}\text { Total } \\
(n=97)\end{array}$ & $\begin{array}{l}\text { Student } \\
\text { patients } \\
(n=48)\end{array}$ & $\begin{array}{l}\text { Student } \\
\text { nurses } \\
(n=49)\end{array}$ \\
\hline \multicolumn{4}{|l|}{ Treatment order } \\
\hline Water and soap bed bath first & $50(52 \%)$ & $25(52 \%)$ & $25(51 \%)$ \\
\hline Washing without water bed bath first & $47(48 \%)$ & $23(48 \%)$ & $24(49 \%)$ \\
\hline \multicolumn{4}{|l|}{ Gender } \\
\hline Female & $83(86 \%)$ & $43(90 \%)$ & 40 (82\%) \\
\hline Male & $14(14 \%)$ & $5(10 \%)$ & $9(18 \%)$ \\
\hline Age in years - mean (SD) & $19.2(1.8)$ & $19(2)$ & $19(2)$ \\
\hline Length in cm - mean (SD) & $171.1(7.2)$ & $171(6)$ & $172(8)$ \\
\hline Weight in $\mathrm{kg}$ - mean (SD) & $65.9(13.2)$ & $65.4(10.8)$ & $66.4(15.3)$ \\
\hline \multicolumn{4}{|l|}{ Has the student been bed bathed before? } \\
\hline Yes & $25(26 \%)$ & $7(15 \%)$ & $18(37 \%)$ \\
\hline No & $72(74 \%)$ & $41(85 \%)$ & $31(63 \%)$ \\
\hline \multicolumn{4}{|l|}{$\begin{array}{l}\text { Has the student provided a bed bath } \\
\text { before? }\end{array}$} \\
\hline Yes & $67(69 \%)$ & $35(73 \%)$ & $32(65 \%)$ \\
\hline No & $30(31 \%)$ & $13(27 \%)$ & $17(35 \%)$ \\
\hline \multicolumn{4}{|l|}{ Preference for bed bathing method } \\
\hline Water and soap bed bath & $28(29 \%)$ & $15(31 \%)$ & $13(27 \%)$ \\
\hline Washing without water bed bath & $14(14 \%)$ & $3(6 \%)$ & $11(22 \%)$ \\
\hline No preference & $55(57 \%)$ & $30(63 \%)$ & $25(51 \%)$ \\
\hline $\begin{array}{l}\text { Perceived importance of the bed bath on a } \\
\text { scale from } 1 \text { to } 10 \text { - mean (SD) }\end{array}$ & $8.7(1.2)$ & $8.7(1.1)$ & 8.7 (1.3) \\
\hline
\end{tabular}


Table 1 provides an overview of the sample's characteristics. The majority of the participants were female, had not been bed bathed before the trial, did have some experience in providing the bed bath, and did not have a preference for a bed bathing method before the trial. Furthermore, participants perceived the bed bath as highly important (from the patient perspective) before the start of the trial.

\section{Primary outcomes}

Emotional and physical comfort of the bed bath were the primary outcomes related to people receiving a bed bath. No statistically significant difference was found between the water and soap method and the washing without water method in the emotional and physical comfort scores among student patients. For those providing the bed bath, only physical comfort was assessed by measuring how physically demanding the bed bath was perceived to be among student nurses. The cumulative physical demands score was statistically significantly lower $(p=.001)$ for the washing without water method $(M=13.24)$ compared to the water and soap method $(M=$ 14.76), which indicates slightly higher physical comfort levels for the washing without water method among student nurses. These results are presented in Table 2 and support our hypotheses.

Additional linear mixed model regressions showed that the order in which the two different bed baths were provided did not have an effect on our primary outcome variables. Furthermore, several covariates were included in the regressions analysis, which did not yield important confounding effects (see appendix 2 for results).

Table 2 - Differences in comfort levels between water and soap bed bath and washing without water

\begin{tabular}{|c|c|c|c|c|c|}
\hline Variable & Bed bath method & Mean (SD) & $p \dagger$ & $95 \% \mathrm{Cl}$ & $\begin{array}{l}\text { Effect size } \\
\text { (Cohen's d) }\end{array}$ \\
\hline $\begin{array}{l}\text { Emotional } \\
\text { comfort student } \\
\text { patients } \ddagger(n=48)\end{array}$ & $\begin{array}{l}\text { Water and soap bed } \\
\text { bath } \\
\text { Washing without water }\end{array}$ & $\begin{array}{l}45.90(5.72) \\
46.06(6.48)\end{array}$ & .847 & $\begin{array}{l}{[-1.89,} \\
1.56]\end{array}$ & - \\
\hline $\begin{array}{l}\text { Physical comfort } \\
\text { student } \\
\text { patients§ }(n=48)\end{array}$ & $\begin{array}{l}\text { Water and soap bed } \\
\text { bath } \\
\text { Washing without water }\end{array}$ & $\begin{array}{l}7.54(1.15) \\
7.50(1.17)\end{array}$ & .828 & $\begin{array}{l}{[-.34} \\
.43]\end{array}$ & - \\
\hline $\begin{array}{l}\text { Physical } \\
\text { demands } \\
\text { student nurses } \mathbb{1} \\
(n=49)\end{array}$ & $\begin{array}{l}\text { Water and soap bed } \\
\text { bath } \\
\text { Washing without water }\end{array}$ & $\begin{array}{l}14.76(3.65) \\
13.24(3.86)\end{array}$ & .001 & $\begin{array}{l}{[.67} \\
2.35]\end{array}$ & Small (0.4) \\
\hline
\end{tabular}

\section{Secondary outcomes}

With respect to the secondary outcomes of this study, statistically significant differences between the two bed bath methods were found on two variables as shown in Table 3. First, student nurses gave a higher general grade $(p=.005)$ to the washing without water method $(M=7.9)$ than to the water and soap method $(M=$ 


\section{Chapter 5}

7.2). Second, a difference was found in bed bath duration $(p<.001)$, with the washing without water method taking less time ( $M=15$ minutes) than the water and soap method ( $M=24$ minutes). No statistically significant difference between the two bed bath methods was found with respect to general grades given by student patients, nor with respect to emotional or physical comfort of the bed bath for student patients according to student nurses.

Table 3 - Results of continuous secondary outcomes

\begin{tabular}{|c|c|c|c|c|c|}
\hline Variable & Bed bath method & Mean (SD) & $p \dagger$ & $\begin{array}{l}95 \% \\
\mathrm{Cl}\end{array}$ & $\begin{array}{l}\text { Effect size } \\
\text { (Cohen's } d \text { ) }\end{array}$ \\
\hline $\begin{array}{l}\text { General grades } \\
\text { given by student } \\
\text { patients to bed } \\
\text { bath method } f \\
(n=48)\end{array}$ & $\begin{array}{l}\text { Water and soap bed } \\
\text { bath } \\
\text { Washing without } \\
\text { water }\end{array}$ & $\begin{array}{l}7.63(1.21) \\
7.38(1.18)\end{array}$ & .308 & $\begin{array}{l}{[-.24} \\
.74]\end{array}$ & - \\
\hline $\begin{array}{l}\text { General grades } \\
\text { given by student } \\
\text { nurses to bed bath } \\
\text { method } \neq(n=49)\end{array}$ & $\begin{array}{l}\text { Water and soap bed } \\
\text { bath } \\
\text { Washing without } \\
\text { water }\end{array}$ & $\begin{array}{l}7.18(1.29) \\
7.86(1.21)\end{array}$ & .005 & $\begin{array}{l}{[-1.13,} \\
-.22]\end{array}$ & $\begin{array}{l}\text { Medium } \\
(0.54)\end{array}$ \\
\hline $\begin{array}{l}\text { Emotional comfort } \\
\text { for student } \\
\text { patients according } \\
\text { to student nurses } \\
(n=49)\end{array}$ & $\begin{array}{l}\text { Water and soap bed } \\
\text { bath } \\
\text { Washing without } \\
\text { water }\end{array}$ & $\begin{array}{l}42.67(5.70) \\
43.04(5.65)\end{array}$ & .592 & $\begin{array}{l}{[-1.74,} \\
1]\end{array}$ & - \\
\hline $\begin{array}{l}\text { Physical comfort } \\
\text { for student } \\
\text { patients according } \\
\text { to student nurses } \\
(n=49)\end{array}$ & $\begin{array}{l}\text { Water and soap bed } \\
\text { bath } \\
\text { Washing without } \\
\text { water }\end{array}$ & $\begin{array}{l}7.04(1.19) \\
7.43(1.17)\end{array}$ & .05 & $\begin{array}{l}{[-.78,} \\
0]\end{array}$ & - \\
\hline $\begin{array}{l}\text { Bed bath duration } \\
(n=49)\end{array}$ & $\begin{array}{l}\text { Water and soap bed } \\
\text { bath } \\
\text { Washing without } \\
\text { water }\end{array}$ & $\begin{array}{l}23.65(5.27) \\
15.06(3.76)\end{array}$ & $<.001$ & $\begin{array}{l}{[7.03} \\
10.15]\end{array}$ & Large (1.88) \\
\hline
\end{tabular}

† Paired samples t-test; † Possible range: 1-10; § Possible range: 12-60.

With respect to preferences among student patients and student nurses, the results in Table 4 reveal that most student patients (48\%) preferred the water and soap method $(p=.028)$ when asked about their general preference. They did not have a statistically significant preference when asked how they wanted to be bathed in a (hypothetical) future trial. The majority of student nurses (63\%) preferred the washing without water method $(p<.001)$ and also chose this bed bath method $(70 \%)$ when they were asked which bed bath they would choose if they had to provide a bed bath again in a future (hypothetical) trial $(p<.001)$. Finally, with respect to the perceived importance of the bed bath, a statistically significant difference between the pre-trial and posttrial scores was only found among student patients $(p=.03$ ) as shown in Table 5 . 
Student patients perceived the bed bath as slightly more important after the trial ( $M$ $=9.0$ ) compared to their perception about its importance before the trial $(M=8.7)$.

Table 4 - Preferences among student patients and student nurses for bed bath methods

\begin{tabular}{|c|c|c|c|c|}
\hline Variable & Preference & $n(\%)$ & $p \dagger$ & $\begin{array}{l}\text { Chi-Square } \\
\text { (df) }\end{array}$ \\
\hline $\begin{array}{l}\text { Preference student } \\
\text { patients ( } n=48 \text { ) }\end{array}$ & $\begin{array}{l}\text { No preference } \\
\text { Water and soap bed bath } \\
\text { Washing without water }\end{array}$ & $\begin{array}{l}8(17 \%) \\
23(48 \%) \\
17(35 \%)\end{array}$ & .028 & $7.13(2)$ \\
\hline $\begin{array}{l}\text { Preference student } \\
\text { nurses }(n=49)\end{array}$ & $\begin{array}{l}\text { No preference } \\
\text { Water and soap bed bath } \\
\text { Washing without water }\end{array}$ & $\begin{array}{l}8(16 \%) \\
10(21 \%) \\
31(63 \%)\end{array}$ & $<.001$ & $19.88(2)$ \\
\hline $\begin{array}{l}\text { Student patients' bed } \\
\text { bath of choice for } \\
\text { future bed bath ( } n=48 \text { ) }\end{array}$ & $\begin{array}{l}\text { No preference } \\
\text { Water and soap bed bath } \\
\text { Washing without water }\end{array}$ & $\begin{array}{l}13(27 \%) \\
19(40 \%) \\
16(33 \%)\end{array}$ & .570 & $1.13(2)$ \\
\hline $\begin{array}{l}\text { Student nurses' bed } \\
\text { bath of choice for } \\
\text { future bed bath ( } n=49 \text { ) }\end{array}$ & $\begin{array}{l}\text { No preference } \\
\text { Water and soap bed bath } \\
\text { Washing without water }\end{array}$ & $\begin{array}{l}7(14 \%) \\
8(16 \%) \\
34(70 \%)\end{array}$ & $<.001$ & $28.69(2)$ \\
\hline
\end{tabular}

† Chi-square goodness-of-fit test assuming equal counts.

Table 5 - Differences in perceived importance of the bed bath before and after the trial

\begin{tabular}{|c|c|c|c|c|c|}
\hline Variable (secondary) & $\begin{array}{l}\text { Before/after } \\
\text { trial }\end{array}$ & Mean (SD) & $p \dagger$ & $95 \% \mathrm{Cl}$ & $\begin{array}{l}\text { Effect size } \\
\text { (Cohen's d) }\end{array}$ \\
\hline $\begin{array}{l}\text { Perceived importance of } \\
\text { the bed bath among }\end{array}$ & $\begin{array}{l}\text { Before the } \\
\text { trial }\end{array}$ & $8.67(1.14)$ & .032 & {$[-.72,-.03]$} & Small (0.35) \\
\hline student patients $\ddagger(n=48)$ & After the trial & $9.04(0.97)$ & & & \\
\hline $\begin{array}{l}\text { Perceived importance of } \\
\text { the bed bath among }\end{array}$ & $\begin{array}{l}\text { Before the } \\
\text { trial }\end{array}$ & $8.78(0.90)$ & .471 & {$[-.31, .14]$} & - \\
\hline student nursesł ( $n=49$ ) & After the trial & $8.86(1.04)$ & & & \\
\hline
\end{tabular}

\section{DISCUSSION}

Our findings indicate that the washing without water method and the water and soap method do not differ in terms of perceived emotional and physical comfort for the person being bathed in bed. A small but statistically significant difference in favor of washing without water was found in perceived physical demands for nurses providing the bed bath. Furthermore, a large difference was found in the duration of the bed bath, which was $36 \%$ shorter for the washing without water method. In addition to emotional and physical comfort, our crossover design made it possible to assess participants' preferences for bed bath methods (i.e. all participants experienced both bed baths), which might relate to perceived comfort levels. Especially among participants that provided the bed baths, a strong preference was detected for the 


\section{Chapter 5}

washing without water method, while participants that were bathed slightly preferred water and soap.

Previous studies have reported care recipients' perceptions about the washing without water concept being soft and convenient, which is positively related to physical comfort. ${ }^{18,27}$ However, our results indicate that there is no difference in physical (nor emotional) comfort between the bed bathing methods for people being bathed. Furthermore, while Schoonhoven et al. reported that most care recipients within a nursing home setting would replace water and soap with washing without water, ${ }^{14}$ our results show a slight preference for the water and soap method among people being bathed. A possible explanation for this finding might be offered by Veje et al. who reported a preference for the water and soap method among care recipients who believed it to be more effective in cleaning the skin. ${ }^{24}$ Although scientific evidence shows that both methods are equally effective in removing microorganisms from the skin, 24,28 this trial's participants might also perceive washing without water as less hygienically effective. Such perceptions might have affected the preferences of the nursing students who participated in this trial as it will be their future responsibility to meet care recipients' hygiene needs.

Our results with respect to users' preferences and perceived physical demand levels are in line with previous research that reported nurses' preferences for the washing without water method for convenience, amongst other reasons related to physical comfort. ${ }^{27-29}$ Also the users in our study mainly preferred the washing without water method and perceived this bed bath as less physically demanding than the water and soap method. Probably, these results are partly explained by the big difference in bed bath duration between the two bed bath methods. We found that the average duration of the bed bath with the washing without water method accounted for less than two-thirds of the duration of a bed bath with water and soap, which is in line with previous research.29, 38

Overall, evidence on washing without water indicates that this bed bath method outperforms or equals the water and soap method with respect to hygiene, skin condition, and bathing completeness, among other areas. ${ }^{26}$ The bed bath method should be selected according to care recipients' preferences but our results indicate that washing without water could especially be valuable for certain groups of care recipients. Washing without water seems highly suitable for patients that would benefit from shorter bed baths, such as people suffering from COPD or people receiving palliative care. Furthermore, people with dementia that show resistance during the bed bath (e.g. because they are afraid of water) could experience more physical and emotional comfort when bathed using the washing without water method. ${ }^{3}$ For nurses, bathing people with dementia can be a struggle, ${ }^{39}$ which also makes washing without water a valuable alternative for them because it takes less time and physical effort. In addition, washing without water can be valuable for healthcare institutions, especially in times of crises, such as the COVID-19 pandemic, when the need for hygienic and comfortable personal care is high but time is limited. Also when time constraints are less pressing, washing without water could free up time 
for nurses to spend on other care activities with care recipients. Furthermore, as the technical execution of the bed bath takes less time with the washing without water method, nurses could spend more time and attention on the interpersonal aspects of the bed bath (e.g. communication with the patient), which we controlled for in our study but can be essential for a comfortable bed bath.

Apart from our results with respect to the bed bath methods, a somewhat remarkable finding can be reported regarding the bed bath in general. Although bathing is reported to be commonly undervalued among nurses and nursing students, ${ }^{40}$ the nursing students that participated in our study assigned high grades to its importance. This might be explained by an above-average interest among this study's participants compared to the much bigger group of nursing students that declined to participate.

Less than $20 \%$ of nursing students were willing to participate in the trial. Possibly, being accustomed to the use of mannequins in practicing health care activities increases students' emotional distress when they personally have to experience the bed bath.41, 42 The common practice of using mannequins could not only have negatively influenced students' willingness to participate in the trial, but in general also deprives students of the possibility to personally experience what it means to be bathed. Therefore, we would like to encourage nursing educators to reintroduce the practice of essential care activities, such as the bed bath, on fellow students. In doing so, we would like to stress the importance of interpersonal factors, such as communication, with respect to how the bed bath (method) is experienced. ${ }^{39}$ In our trial, the person providing the bed bath and communication during the bed bath were potential confounders, which we controlled for by making fixed student couples and by instructing participants to confine communication during the bed baths. However, in actual health care settings, these interpersonal factors influence health care actors' experiences, including comfort perceptions. ${ }^{39,} 43$ Therefore, nursing educators should also pay attention to such factors to offer valuable learning experiences to students. If students obtain personal and more realistic experience with the bed bath, they will likely possess more knowledge and confidence and struggle less in properly carrying out bed baths on real patients. 4, 5, 22, 44, 45 Moreover, practicing on real people and personally experiencing the bed bath as a patient adds to students' understanding about the importance of this essential care activity.

Bathing people (i.e. instead of mannequins) in different ways also supports students to form better informed judgements with respect to bathing interventions, such as washing without water. Generally, there is a lack of evidence on essential care interventions that could support nurses to deliver optimal care. ${ }^{5}$ Our study contributes to the limited scientific evidence on bathing, which hopefully supports the reevaluation of its importance. Moreover, our results add to the evidence base regarding washing without water and justify its use in health care practice from a comfort perspective. 


\section{Limitations and recommendations for future research}

To our knowledge, our study is the first to assess differences between the water and soap method and the washing without water method with respect to comfort, which is one of the foremost goals of bed bathing. ${ }^{18}$ Although this study has been carried out carefully, some limitations need to be mentioned that should be taken into account in future research.

Three limitations are related to our sample. First, $93 \%$ of the calculated sample size was reached, which was partly due to the study design. Only participants that completed both treatments were included in the analysis. Because couples of students were created, this meant that both students had to be excluded from the analyses if one of the two did not show up on the second day of the trial. However, the attrition rate in our study (13\%) was below $20 \%$, which is argued to be the level at which bias in the results can be expected. 42 Still, our study might be underpowered, especially because participants were eventually recruited from four clusters of nursing students of two nursing schools, which was needed due to the low participation rate. The low participation rate implies a second limitation as it could have caused participation bias because the participating students might have different perceptions about the bed bath compared to the students who declined to participate in the trial. Third, while this study's laboratory setting is suitable for having a first check on causality and therefore, has a positive effect on the internal validity of our findings, 32,46 the chosen setting and study subjects (i.e. nursing students) have implications for our findings' external validity. Students differ in many ways from actual bedridden care recipients, and form a much more homogenous group. ${ }^{47}$ Furthermore, because interpersonal factors (e.g. communication) that could influence comfort experiences were controlled for in the current trial, our results related to student patients' comfort perceptions and preferences should cautiously be generalized to actual bedridden care recipients. Therefore, future research should assess comfort perceptions in real-life health care settings with actual bedridden care recipients, such as nursing home residents or hospital patients, taking into account interpersonal factors. ${ }^{47}$

Yet, our results with respect to student nurses' perceptions and preferences can be generalized to actual nurses providing real bed baths. Wilson et al. argue that experiments that score high on experimental and mundane realism can still have high external validity. ${ }^{32}$ Experimental realism is the extent to which the experiment is involving for the participants and has an impact on them, whereas mundane realism is the extent to which the experimental situation is likely to occur in the participants' real lives. For the student patients in our study, experimental realism was likely high, but mundane realism rather low as they did not need to be bathed by others in real life. However, for student nurses, both types of realism are likely to be high because these students will provide bed baths to actual bedridden people in the near future.

Another limitation relates to the measurement of physical comfort for which we found a surprisingly limited number of validated scales. Although it is a rather general construct, we mainly found scales related to pain or broad comfort scales 
that do not measure physical comfort specifically. 48,49 Consequently, we measured physical comfort among student patients with a single-item question, and used the Physical Demands scale as a proxy for physical comfort among student nurses. While high reliability has been reported for the Physical Demands scale, 34,35 its reliability turned out to be rather low in the current study (Cronbach's alpha coefficients of .58 for the water and soap method and .72 for the washing without water method). Conversely, the reliability of the PEECE scale, which was used to measure emotional comfort, was high (Cronbach's alpha coefficients of .87 and .89). These results should be taken into account in the selection of scales to measure comfort in future research. Furthermore, we focused on emotional and physical comfort, but comfort is often described as a holistic construct including physical, psychospiritual, environmental, and social dimensions. ${ }^{50}$ Therefore, future research could study differences in other comfort dimensions between the different bed bath methods.

\section{CONCLUSION}

Based on our results, washing without water seems to be a suitable alternative for the traditional bed bath with water and soap from the care recipient's comfort perspective. Moreover, washing without water is more time-efficient and is less physically demanding for those providing the bed bath. Therefore, its use in health care practice is encouraged.

\section{RELEVANCE TO CLINICAL PRACTICE}

To follow the daily natural routine, bed baths are mainly provided in the morning, which puts time constraints on to nursing staff. Our study results show that washing without water is less physically demanding for nurses, which is related to nurses' physical comfort. In addition, the washing without water concept saves time without having a detrimental effect on care recipients' emotional and physical comfort. Nurses could spend the time saved on other care activities with the care recipient. Finally, our results inform health care practice and educators about the perceptions regarding bed bath methods among nursing students, who will soon have to carry out these bed baths themselves. 


\section{References}

1. Jagger C, Arthur AJ, Spiers NA, et al. Patterns of onset of disability in activities of daily living with age. Journal of the American Geriatrics Society 2001;49:404-409.

2. Eurostat. Disability statistics prevalence and demographics. 2016. Available from: https://ec.europa.eu/eurostat/statistics-explained/pdfscache/34409.pdf.

Accessed 3 Oct 2019.

3. Cohen-Mansfield $\mathrm{J}$ and Jensen B. The preference and importance of bathing, toileting and mouth care habits in older persons. Gerontology 2005;51:375-385.

4. Feo R and Kitson A. Promoting patient-centred fundamental care in acute healthcare systems. International Journal of Nursing Studies 2016:57:1-11.

5. Zwakhalen SMG, Hamers JPH, Metzelthin SF, et al. Basic nursing care: The most provided, the least evidence based. A discussion paper. Journal of Clinical Nursing 2018;27:2496-2505.

6. Groven FMV, Odekerken-Schröder G, Zwakhalen SMG, et al. Network Well-Being from a Balanced Centricity Perspective. Journal of Services Marketing, 2021;35:114.

7. Jangland E, Mirza N, Conroy $T$, et al. Nursing students' understanding of the Fundamentals of Care: A cross-sectional study in five countries. Journal of Clinical Nursing 2018;27:2460-2472.

8. Pipe TB, Connolly T, Spahr N, et al. Bringing back the basics of nursing: defining patient care essentials. Nursing Administration Quarterly 2012;36:225-233.

9. Parr JM, Bell J and Koziol-McLain J. Evaluating fundamentals of care: The development of a unit-level quality measurement and improvement programme. Journal of Clinical Nursing 2018;27:2360-2372.

10. Lorente S, Losilla JM and Vives J. Instruments to assess patient comfort during hospitalization: A psychometric review. Journal of Advanced Nursing 2017;74: 1001-1015.

11. Malinowski A and Stamler LL. Comfort: exploration of the concept in nursing. Journal of Advanced Nursing 2002;39:599-606.

12. Williams AM and Irurita VF. Therapeutic and non-therapeutic interpersonal interactions: The patient's perspective. Journal of Clinical Nursing 2004;13:806-815.

13. Kolcaba K. A theory of holistic comfort for nursing. Journal of Advanced Nursing 1994;19:1178-1184.

14. Schoonhoven L, van Gaal, BGl, Teerenstra S, et al. Cost-consequence analysis of "washing without water" for nursing home residents: a cluster randomized trial. International Journal of Nursing Studies 2015;52:112-120.

15. Richards DA, Hilli A, Pentecost C, et al. Fundamental nursing care: A systematic review of the evidence on the effect of nursing care interventions for nutrition, elimination, mobility and hygiene. Journal of Clinical Nursing 2018;27:2179-2188.

16. Downey L and Lloyd H. Bed bathing patients in hospital. Nursing Standard 2008;22:35-40.

17. Lopes JL, Nogueira-Martins LA and de Barros ALBL. Bed and shower baths: comparing the perceptions of patients with acute myocardial infarction. Journal of Clinical Nursing 2012;22:733-740.

18. Veje PL, Chen M, Jensen CS, et al. Bed bath with soap and water or disposable wet wipes: Patients' experiences and preferences. Journal of Clinical Nursing 2019;28:2235-2244. 
19. Morse JM, Bottorff JL and Hutchinson S. The paradox of comfort. Nursing Research 1995:44:14-19.

20. Johannesen A, Petersen $J$ and Avlund K. Satisfaction in everyday life for frail 85year-old adults: a Danish population study. Scandinavian Journal of Occupational Therapy 2004;11:3-11.

21. Rader J, Barrick AL, Hoeffer B, et al. The bathing of older adults with dementia: easing the unnecessarily unpleasant aspects of assisted bathing. AJN The American Journal of Nursing 2006;106:40-48.

22. El-Soussi AH and Asfour HI. Examining bed-bath practices of critically ill patients. Journal of Nursing Education and Practice 2016;12:1-11.

23. Hoeffer B, Talerico KA, Rasin J, et al. Assisting cognitively impaired nursing home residents with bathing: effects of two bathing interventions on caregiving. Gerontologist 2006;46:524-532.

24. Veje PL, Chen M, Jensen CS, et al. Effectiveness of two bed bath methods in removing microorganisms from hospitalized patients: A prospective randomized crossover study. American Journal of Infection Control 2020;48:638-643.

25. Sturgeon LP, Garrett-Wright D, Lartey G, et al. A descriptive study of bathing practices in acute care facilities in the United States. American Journal of Infection Control 2019;47:23-26.

26. Groven FMV, Zwakhalen S, Odekerken-Schröder G, et al. How does washing without water perform compared to the traditional bed bath: A systematic review. BMC Geriatrics 2017;17:1-16.

27. Sheppard CM and Brenner PS. The effects of bathing and skin care practices on skin quality and satisfaction with an innovative product. Journal of Gerontological Nursing 2000;26:36-45.

28. Larson EL, Ciliberti T, Chantler C, et al. Comparison of traditional and disposable bed baths in critically ill patients. American Journal of Critical Care 2004;13:235241.

29. Nøddeskou LH, Hemmingsen L and Hørdam B. Elderly patients' and nurses' assessment of traditional bed bath compared to prepacked single units randomised controlled trial. Scandinavian Journal of Caring Sciences 2015;29:347352.

30. Groven FMV, Zwakhalen SMG, Odekerken-Schröder G, et al. The effects of washing without water versus the traditonal bed bath with water and soap on comfort and physical demands: protocol of a cross-over randomized trial. 2019. Available from: https://assets.researchsquare.com/files/rs7409/v1/manuscript.pdf.

31. Richards DA and Hamers JPH. RCTs in complex nursing interventions and laboratory experimental studies. International Journal of Nursing Studies 2009;46:588-592.

32. Wilson TD, Aronson E and Carlsmith K. The art of laboratory experimentation. In S. T. Fiske, D. T. Gilbert, \& G. Lindzey (Eds.). 2010. Handbook of social psychology. Volume one (pp. 51-81). New Jersey: John Wiley \& Sons.

33. Williams AM, Lester L, Bulsara $C$, et al. Patient Evaluation of Emotional Comfort Experienced (PEECE): developing and testing a measurement instrument. BMJ Open 2017:7:7. 
34. De Jonge J, Mulder MJGP and Nijhuis FJN. The incorporation of different demand concepts in the job demand-control model: Effects on health care professionals. Social Science \& Medicine 1999:48:1149-1160.

35. Verbeek H. Redesigning dementia care - An evaluation of small-scale, homelike care environments. 2011 (Doctoral dissertation).

36. Tan FES, Jolani S and Verbeek H. Guidelines for multiple imputations in repeated measurements with time-dependent covariates: A case study. Journal of Clinical Epidemiology 2018;102:107-114.

37. Cohen J. Statistical power analysis for the behavioral sciences. 1977. London: Academic Press.

38. Nøddeskou LH, Túgvustein N, Marjunardóttir A, et al. Assessment of bed bathing methods in the Faroe Islands. The American Journal of Nursing Science 2018;7:109114.

39. Conroy T. Factors influencing the delivery of the fundamentals of care: Perceptions of nurses, nursing leaders and healthcare consumers. Journal of Clinical Nursing 2017;27:2373-2386.

40. Meehan TC, Timmins F and Burke J. Fundamental care guided by the Careful Nursing Philosophy and Professional Practice Model@. Journal of Clinical Nursing 2018;27:2260-2273.

41. Wolf ZR. Nursing students' experience bathing patients for the first time. Nurse Educator 1997;22:41-46.

42. Gul RB and Ali PA. Clinical trials: The challenge of recruitment and retention of participants. Journal of Clinical Nursing 2010;19:227-233.

43. Miranda RPR, de Cássia Lopes Chaves É, Lima RS, et al. The effectiveness of a simulated scenario to teach nursing students how to perform a bed bath - a randomized clinical trial. Nurse Education Today 2017:57:17-23.

44. Lopes JL, Baptista RCN, Lopes CT, et al. Efficacy of a video during bed bath simulation on improving the performance or psychomotor skills of nursing undergraduates: A randomized clinical trial. International Journal of Nursing Studies 2019:99:7-12.

45. Feo R, Kitson A and Conroy T. How fundamental aspects of nursing care are defined in the literature: A scoping review. Journal of Clinical Nursing 2018;27:21892229.

46. Hooghe M, Stolle D, Mahéo V, et al. Why can't a student be more like an average person? Sampling and attrition effects in social science field and laboratory experiments. The Annals of the American Academy 2010;628:85-96.

47. Andersen S, Harrison GW, Lau Ml, et al. Preference heterogeneity in experiments: Comparing the field and laboratory. Journal of Economic Behavior \& Organization 2010;73:209-224.

48. Kolcaba K, Schirm V and Steiner R. Effects of hand massage on comfort of nursing home residents. Geriatric Nursing 2006;27: 85-91.

49. Leonardsen AL, Grøndahl VA, Ghanima W, et al. Evaluating patient experiences in decentralised acute care using the Picker Patient Experience Questionnaire; methodological and clinical findings. BMC Health Services Research 2017;17.

50. Kolcaba K. Holistic comfort: Operationalizing the construct as a nurse-sensitive outcome. Advances in Nursing Science 1992;15:1-10. 
Appendix 1 - Cronbach's alpha coefficients PEECE scale and Physical Demands scale

\begin{tabular}{lll}
\hline & $\begin{array}{l}\text { Cronbach's alpha values (mean inter-item } \\
\text { correlation) }\end{array}$ & Washing without water \\
\hline Scale & Traditional bed bath & 0.886 \\
\hline $\begin{array}{l}\text { PEECE measuring emotional } \\
\text { comfort of patients during the } \\
\text { bed bath (12 items) }\end{array}$ & 0.874 & \\
& & 0.719 \\
$\begin{array}{l}\text { Physical Demands measuring } \\
\text { physical demands of the bed } \\
\text { bath among nurses (7 items) }\end{array}$ & 0.581 & $(0.152)$ \\
\hline
\end{tabular}




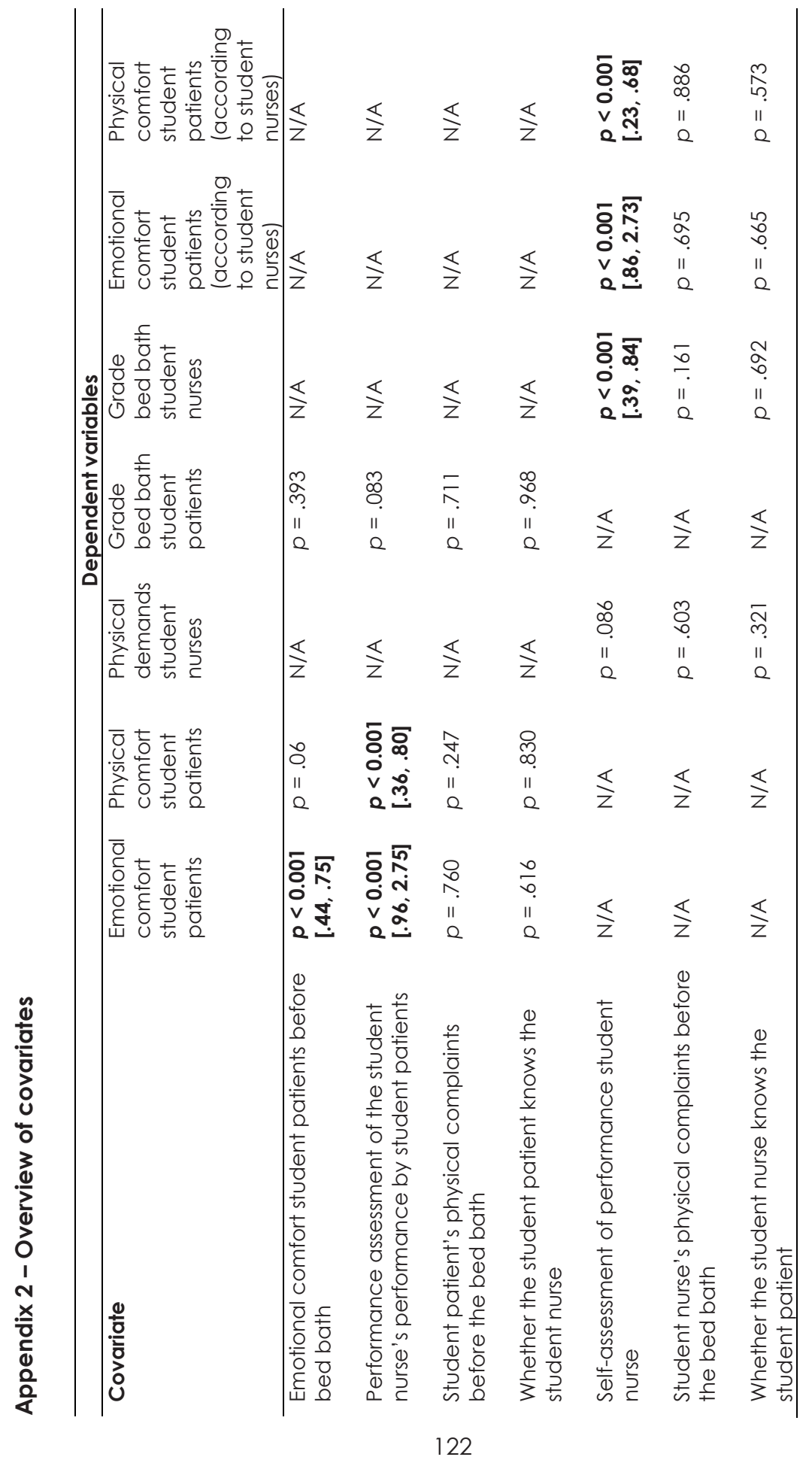


chapter

6
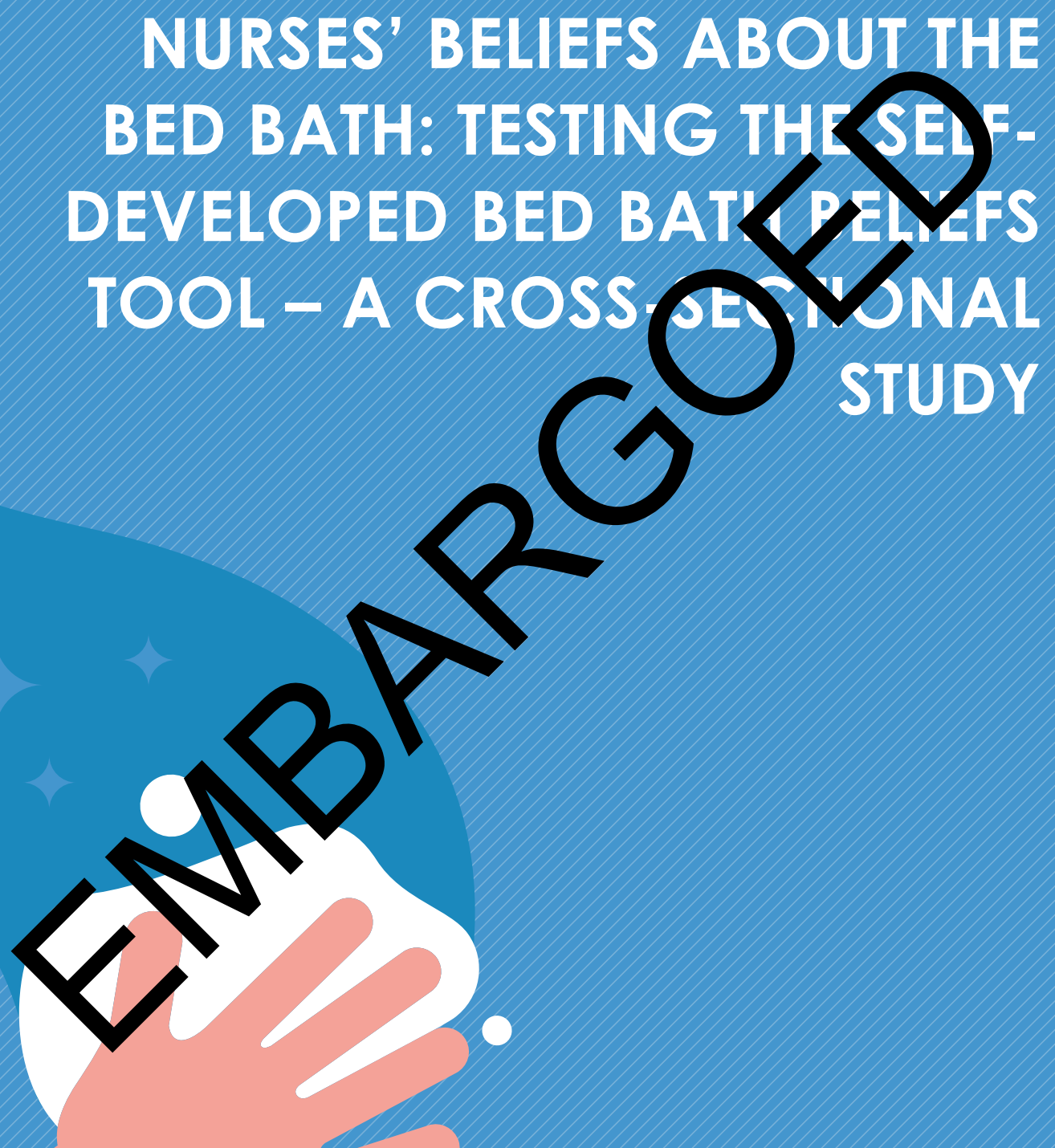

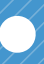


chapter

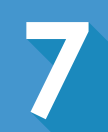

\section{GENERAL DISCUSSION}




\section{Chapter 7}

The bed bath is an important activity, amongst others as a nursing activity that contributes to patient well-being and as a service encounter in which value is cocreated. The aim of this dissertation was to assess the suitability of the washing without water intervention for the bed bath based on the experiences of health care actors, which reflect the well-being of these actors. The central research question was:

What is the effect of the washing without water intervention on health care actors' experiences and well-being, within the scope of the bed bath?

\section{MAIN FINDINGS}

Overall, the main findings of the studies in this dissertation are that: 1) the bed bath is a very important health care activity because of its impact on the well-being of patients, nurses, and, although to a lesser extent, family members and 2) washing without water is a suitable alternative for the water and soap bed bath. An illustrative overview of the main results is provided in Figure 1.

The experiences of the nursing home residents, nurses, and family members interviewed for the qualitative study (Chapter 2) emphasize the importance of the bed bath. The narratives of nursing home residents and nurses illustrate how the bed bath affects their well-being during and after the bed bath. Aside from the importance of the bed bath in general, several study findings presented in this dissertation show that the washing without water intervention is a suitable bed bathing alternative to the traditional bed bath with water and soap. The results of the systematic review (Chapter 3) show that washing without water performs similar to or better than the traditional bed bath on all of the studied outcome measures. No difference between the washing without water bed bath and the traditional bed bath was found with respect to significant skin lesions (i.e. open wounds), costs, microbial count on the skin after a bed bath, resistance during bathing in patients with dementia, and bed bath quality. Moreover, a difference in favor of the washing without water bed bath was found for skin abnormalities (i.e. minor skin deficiencies such as red or flaky skin), skin dryness and skin hydration, nursing staff satisfaction, bathing completeness and time duration of the bed bath. Results related to comfort were not reported in the systematic review, despite comfort representing an important bed bath outcome. Therefore, comfort was studied in the crossover randomized controlled trial (RCT) presented in Chapter 5, which shows that washing without water is less physically demanding for persons providing the bed bath and is as emotionally and physically comfortable as the water and soap bed bath for people being bathed in bed. The crossover RCT also confirmed that the washing without water bed bath was shorter compared to the traditional bed bath, which could add to the value of washing without water for nurses, especially when they face high workloads. 


\section{THE BED BATH ACTIVITY IN GENERAL}
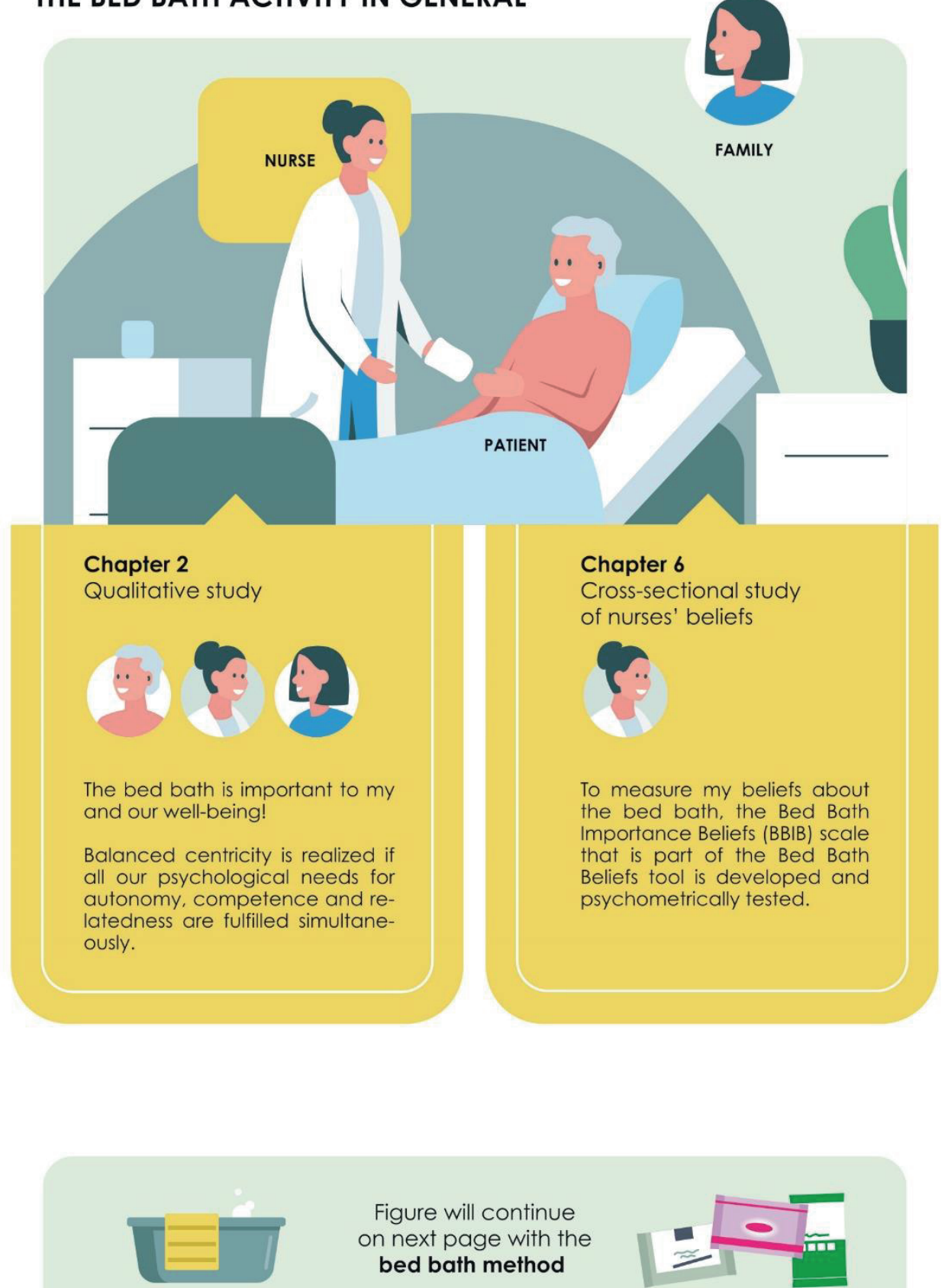

Figure will continue on next page with the bed bath method

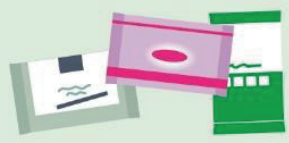

Figure 1 - Illustration of the main findings 


\section{THE BED BATH METHOD}

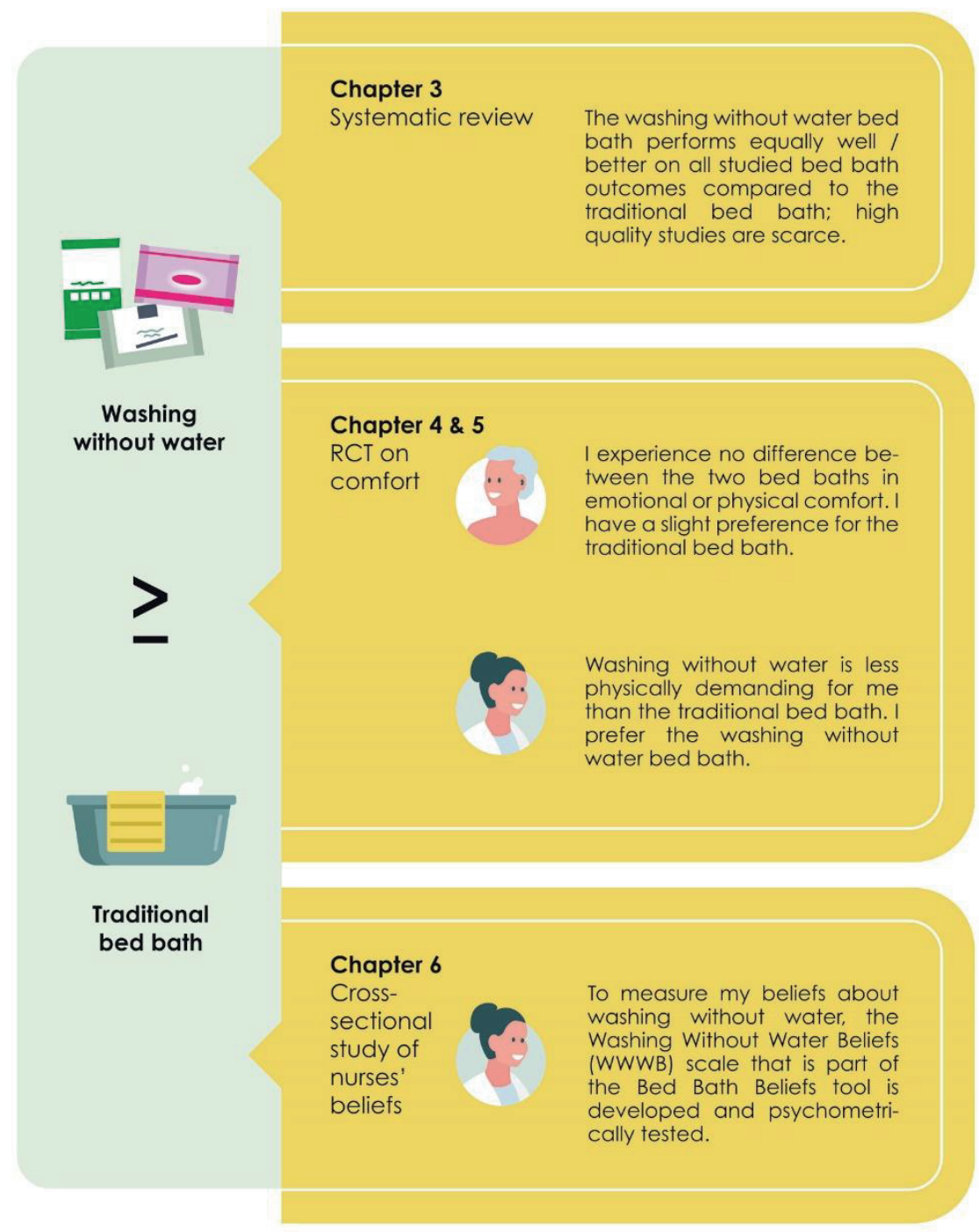

\section{DISCUSSION}

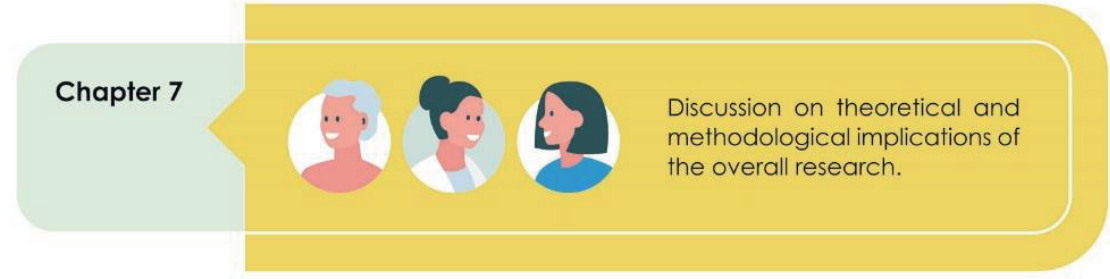

Figure 1 (continued) - Illustration of the main findings 


\section{THEORETICAL IMPLICATIONS}

Three theoretical topics related to this dissertation will be discussed in this section: the added value of the multidisciplinary research setup, self-determination theory (SDT) as the theoretical context for the bed bath, and possible implications of the use of washing without water for the psychological needs of autonomy, competence and relatedness.

\section{Multidisciplinary research setup}

The combination of different scientific disciplines contributes to interdisciplinary knowledge that is valuable for several disciplines.' Combining scientific disciplines leads to the accumulation of knowledge and contributes to a composite (and more realistic) picture of a certain phenomenon. ${ }^{2}$ That is, by looking at a phenomenon (e.g. the bed bath) through different disciplinary lenses, a dialogue between the disciplines evolves and complementary knowledge can be developed to address a common goal. ${ }^{1-3}$ Contributing to well-being is argued to be a common goal that has the potential to unite different disciplines. ${ }^{4}$ Therefore, the deliberate choice was made to take a multidisciplinary research approach to study the effects of the bed bath and washing without water on the experiences and well-being of health care actors.

Actor well-being is important to both the service (marketing) science and the nursing science disciplines. The general goal of nursing science is to enhance health and well-being, primarily of patients. ${ }^{5}$ Well-being is also becoming increasingly important in service science, with the rise of transformative service research (TSR). 6 Furthermore, both disciplines share some theoretical underpinnings related to wellbeing. First, the importance of actor experiences with respect to well-being is recognized by both disciplines. -10 $^{-10}$ ased on the common emphasis on experiences, comfort was selected as the dependent outcome in the crossover RCT (Chapter 5) as a highly experiential variable. Second, both the service science and the nursing science disciplines recognize the importance of the interplay and collaboration between different health care actors with respect to well-being. 11, 12 Third, SDT, and the importance of the psychological needs for well-being (Chapter 2) are recognized in both disciplines.

In addition to the common theoretical underpinnings, each discipline has its own theoretical lens to look at well-being. ${ }^{5}$ In service marketing, well-being is heavily interrelated to value, which is a surprisingly underrepresented concept in nursing literature. However, in nursing science, well-being is related to constructs such as quality of life or health, ${ }^{13}$ which are related to value although not framed as such. ${ }^{14}$ Furthermore, service science often relates well-being to the abstract value cocreation view that can be applied to many service contexts, including health care. ${ }^{15}$ In the value co-creation view, all actors are value co-creators that aim to create value for themselves and others through value co-creation activities and interactions. ${ }^{16}$ The importance of such activities and interactions is also recognized in nursing science, however, not from a value co-creation perspective but rather in 
relation to concepts important to nursing science, such as patient involvement/participation. ${ }^{17}$

By combining the theoretical lenses of service science and nursing science, valuable contributions are made to both disciplines. ${ }^{18,} 19$ To service science, the bed bath offered a valuable "case" to study value co-creation dynamics, which contributed to the theoretical concept of balanced centricity, which is achieved if all actors' needs are fulfilled. ${ }^{16}$ The application of the value co-creation lens at the same time complemented nursing science by providing another perspective to look at the bed bath as a value co-creation activity that affects the well-being of patients, nurses, and family members.

\section{Self-determination theory as the theoretical context for the bed bath}

SDT is a theory on motivation, which presents the universal and innate human psychological needs of autonomy, competence, and relatedness as determinants of daily well-being, and is known in both the service science and the nursing science disciplines. ${ }^{20}$ SDT emerged as a suitable theory in the data analysis process of the qualitative study (Chapter 2) because the needs of the interviewed health care actors all related to these psychological needs. The psychological needs central to SDT could serve as a mid-range nursing theory that connects SDT to nursing practice, in our case to the nursing activity of the bed bath. In nursing, mid-range theories have been introduced to inform nurses about the implications of nursing theories for their thinking and acting in health care practice. 10, 21, 22 The psychological needs addressed in this dissertation could be considered mid-range theoretical concepts, because they inform nurses how to contribute to well-being by paying attention to the psychological needs of autonomy, competence and relatedness in essential nursing activities, such as the bed bath.

By connecting theory (i.e. SDT) to nursing practice (i.e. the bed bath), we contribute to the advancement of nursing and add to nursing practice and theory. ${ }^{23}$ First, we contribute to nursing practice by offering easy-to-use and relevant theoretical concepts (i.e. the psychological needs) that can be applied to essential care activities, such as the bed bath. Consequently, we bridge the theory-to-practice gap by making research-based recommendations that support the betterment of health care actors. ${ }^{22,} 24$ By applying SDT to the bed bath, the bed bath is not only a task-oriented care activity but also an activity that can contribute to patients' (and other actors') well-being by fulfilling their psychological needs of autonomy, competence and relatedness. ${ }^{10}$ Consequently, we fill the void in evidence on the bed bath that could guide nurses to provide quality nursing care and thereby uplift the importance of the bed bath. ${ }^{12}$ Furthermore, SDT offers another perspective to essential care activities. For example, autonomy is not only an end goal for nursing (e.g. as advocated by Henderson ${ }^{25}$ ) but also an important determinant for well-being according to SDT, which nurses can focus on when providing essential care.

By applying SDT to the bed bath, we also made some theoretical contributions. We showed that the psychological needs of SDT can be manifested in 
several lower-order needs that differ between actors and possibly also across activities. Lower-order needs with respect to the bed bath might well be different from lower-order needs related to other nursing activities, such as feeding or dressing. By identifying the lower-order needs of actors, we refined the theoretical concepts of the psychological needs. ${ }^{23}$ Furthermore, we showed that the psychological needs are important for several actors' well-being. Finally, while nursing science pays a lot of attention to autonomy with respect to patient well-being, our findings show that the other psychological needs are important too.

\section{Implications of the use of washing without water for psychological needs}

Our findings with respect to washing without water (Chapter 3 and 5) are aligned with nursing science's focus on evidence-based practice in relation to nursing interventions. As a mid-range theory, the psychological needs that are central to SDT could be applied as a theoretical context for these findings. 10 The psychological needs were identified based on actor experiences with the bed bath in general in Chapter 2; specific experiences with the washing without water bed bath were not assessed. If the use of washing without water contributes to the fulfillment of actors' psychological needs, the well-being of these individual actors will increase. ${ }^{26}$ Moreover, if the psychological needs of patients, nurses and family members are fulfilled simultaneously by the use of washing without water, we postulate that balanced centricity and consequently network well-being increases.27, 28 Therefore, we will relate our findings on washing without water with each of the psychological needs in the remainder of this theoretical discussion. The need for autonomy is particularly considered from the patient's perspective, the need for competence is mainly discussed from the nurse's perspective, and we take the patient-nurse dyad perspective in the discussion regarding the need for relatedness.

The use of washing without water in relation to the autonomy of patients

Autonomy means that actors need to experience control and the ability to selforganize experiences and activities. ${ }^{29,} 30$ Autonomy is vital for individuals to live their lives according to their own choices, goals and interests. ${ }^{31}$ Patients' vulnerability and dependence, as well as people's perceptions about patients' inability to make autonomous decisions, puts their autonomy at risk. ${ }^{31}$ Therefore, we focus on patient autonomy with respect to the bed bath, which entails their need for controlling the bed bath, in other words, to have a say about its execution. Related to autonomy, we will discuss the results about patients' bed bath preferences and the added value of having a choice with respect to the bed bath.

The use of washing without water supports patient autonomy when it is in line with patients' preferences. Our findings provide mixed results regarding patients' bed bath method preferences. On the one hand, washing without water is seen as a replacement or a valuable alternative to the traditional bed bath across patients in studies that are included in the systematic review (Chapter 3). On the other hand, the results of our crossover RCT (Chapter 5) indicate a slight preference for the traditional 


\section{Chapter 7}

bed bath among the students that were bathed in bed as a patient, which might be explained by a perceived necessity of water to clean the skin and freshen up.32 Patients that question the hygienic effectiveness of washing without water will likely feel more "fresh" after a traditional bed bath and therefore could prefer such a bed bath.

Furthermore, patients' bed bath method preferences are situation bound as circumstantiated by our qualitative study (Chapter 2), which was not about specific bed bathing methods but nevertheless identified patients' experiences with the washing without water bed bath. Nursing home residents that were interviewed did not express negative experiences with the washing without water bed bath and some explicitly shared their preference for this type of bed bath. However, some patients talked about situations in which they want to have a traditional bed bath. Because bed bath preferences are not stable, preferences should be assessed for each individual patient to support patient autonomy, preferably every time a bed bath is provided. 33

Apart from having a choice in bed bath methods, patient autonomy can be supported if patients can control the time of the bed bath. The use of washing without water could enhance patient autonomy if it allows patients to bathe themselves (partly) and therefore to have control over the time of the bed bath. Washing without water probably is more suitable to be used by patients themselves than the traditional bed bath, which for example requires the collection of many bathing materials.

Unfortunately, many patients actually have little choice in morning care, ${ }^{33}$ also with respect to the bed bath. ${ }^{34}$ Nurses might not offer patients the option to have a washing without water bed bath (or to bathe themselves) because of paternalistic and routinized care approaches. ${ }^{31}$, 35, 36 However, we did not find differences between the two bed bathing methods regarding important outcomes, such as microbial counts on the skin (Chapter 3) or patient comfort (Chapter 5). Moreover, the differences found were in favor of washing without water, such as skin condition, bathing completeness (Chapter 3), physical comfort for the nurse, and the duration of the bed bath (Chapter 5). Therefore, nurses can and should offer patients a choice between a traditional and a washing without water bed bath based on our results. Patients that are offered a choice in bed bath methods are likely to perceive the bed bath as more supportive of autonomy, which contributes to the autonomy of patients. ${ }^{37}$

Although autonomy needs have been identified for nurses with respect to the bed bath and even though autonomy in general is important for nurses with respect to their job satisfaction, 38 the qualitative study (Chapter 2) did unveil that the competence-need of nurses to be a professional nurse prevails. The possible effects of the use of washing without water on nurses' need for competence is therefore discussed next. 
The use of washing without water in relation to nurses' competence

SDT presents competence as the need to experience proficiency in executing tasks, physically and socially. ${ }^{29}, 30$ With respect to the bed bath, we identified competencerelated needs for patients (i.e. to be proficient in daily life), nurses (i.e. to be proficient as a health care specialist), and family members (i.e. to be proficient as an informal caregiver). We will focus on the need of nurses to be a proficient health care professional, and how this could be affected by the use of washing without water by discussing the results regarding (perceived) hygienic effectiveness and nurses' beliefs about the washing without water bed bath. Furthermore, we will touch upon the general importance of the bed bath.

Nurses' need for competence regarding the bed bath is supported if they perceive to have provided a proper bed bath that meets professional standards. However, some nurses appear to question the appropriateness of the washing without water bed bath as reflected in our beliefs study (Chapter 6). The belief statements about washing without water with the lowest level of agreement across nurses were about feeling satisfied after having provided a bed bath with washing without water and about the smell of patients after a washing without water bed bath. Furthermore, some of the nurses that were interviewed for our qualitative study (Chapter 2) indicated that they feel they failed to offer a proper bed bath if no water was used. Consequently, nurses that hold doubts about the suitability of washing without water may refrain from offering patients such a bed bath as that would make nurses feel incompetent. ${ }^{36}$

Such doubts are understandable due to cultural habits but not substantiated by our findings, which show that the washing without water bed bath fits professional nursing levels. For example, the systematic review (Chapter 3) found no difference in bed bath quality between the two bed bathing methods. Other results of the systematic review show the microbial count on the skin after a bed bath is equal for both bed bath methods, and patients are more often bathed completely when washing without water is used. Therefore, nurses can feel assured that washing without water is a viable option to offer patients. Moreover, washing without water can be especially valuable, and support the competence needs of nurses, when nurses face high workloads. Because a washing without water bed bath takes less time (Chapter 5) or even can be executed by some patients themselves, the use of washing without water might prevent the omission of bed baths, which unfortunately sometimes occurs in health care practice. 39,40 Hence, washing without water could support the competence needs of nurses if it enables nurses to ensure bed baths for all patients.

If we zoom out a bit, our results underline the general importance of the bed bath, regardless of how it is executed. Therefore, the act itself of providing the essential care activity of the bed bath should already add to the need of nurses to be proficient health care specialists and consequently contribute to nurses' need for competence. Although nurses have been reported to often undervalue the bed bath,41 participants of our crossover RCT (Chapter 5) gave very high grades for the importance of the bed bath before the start of the trial (around 8.7 on a scale from 1 


\section{Chapter 7}

to 10). Furthermore, the beliefs that were measured in the cross-sectional study (Chapter 6) also indicate a high perceived importance of the bed bath. These findings signal that the undervaluation of the bed bath might not be as substantial as assumed.

The use of washing without water in relation to relatedness (for the patient-nurse dyad)

The SDT need of relatedness is about the need to feel belonged and the need for safe and trusted relationships, 30,42 which are also central to relationship-centered care. ${ }^{35}$ Generally, opportunities to build pleasant and safe relationships that contribute to a sense of belonging and patients' quality of life are important but scarce in health care practice. ${ }^{43,44}$ Because nurses spend most of their time in personal care activities, such as bathing, these activities are perceived as important relational encounters. With respect to the bed bath, both patients and nurses have the need for safe and pleasant relationships with each other (Chapter 2). Important conditions for safe and pleasant relationships to emerge that can be influenced by the use of washing without water are time and comfort during the bed bath.

Because the washing without water bed bath was found to be shorter in duration (and therefore, less time was spent with the patient during the bed bath), washing without water might be perceived to negatively impact patients' and nurses' need for relatedness (i.e. the opportunity to build a safe and pleasant relationship). However, previous research shows that the quantity and quality of communication during activities, such as the bed bath, is already quite limited due to time constraints. ${ }^{35,43}$ The time efficiency of the washing without water bed bath therefore might even free up time and possibilities for higher quality interactions between patients and nurses to emerge. Furthermore, the use of washing without water could enable nurses to provide a proper bed bath to more patients within the time that is available to them, which means that more patients could get a proper bed bath (and personal contact) than before. Consequently, the need for relatedness of more patients can be supported.

Apart from time, emotional and physical comfort levels of patients and nurses are likely to affect the emergence of safe and pleasant relationships, as is also reflected in narratives collected for the qualitative study (Chapter 2). For example, some nurses described relational struggles with patients that displayed negative moods. Although most patients probably want to finish the bed bath as convenient and as fast as possible due to the vulnerable and dependent position they are in, nurses should still strive for optimal patient physical and emotional well-being during the bed bath. The results of our crossover RCT (Chapter 5) showed no difference in patients' emotional or physical comfort levels between the washing without water bed bath and the traditional bed bath. Furthermore, the systematic review (Chapter 3) found no difference in resistance during the bed bath among patients with dementia, which could signpost discomfort. 45 
With respect to the physical comfort of nurses, washing without water was found to be less physically demanding, which could positively affect the ability of nurses to establish safe and pleasant relationships with their patients. That is, nurses' experiences (e.g. comfort experiences) influence the content and process of the interactions nurses have with patients, which in turn influences patients' experiences. ${ }^{46}$ Furthermore, the use of washing without water could contribute to nurses' needs for relatedness if subsequent time savings could be invested in other activities with the patient. That is, nurses who have opportunities to spend more time with patients in physical and cognitive activities (other than personal care activities) are reported to have higher levels of job satisfaction. ${ }^{43}$ This effect is even stronger if nurses are assigned to particular patients, because they can then build trusted relationships in which they can see the effects of their actions on patient well-being, ${ }^{25}$ which also contributes to relationship-centered care. ${ }^{35}$

\section{METHODOLOGICAL IMPLICATIONS}

Methodological implications of the overall dissertation related to scientific integrity and validity will be discussed in this section.

\section{Scientific Integrity}

Integrity means that a moral and ethical code is respected in all phases of the research project and in all interactions with others that are part of the research process. ${ }^{47}$ The research project presented in this dissertation shows that scientific integrity and industry-funded research can go hand-in-hand. ${ }^{48}$ The principles presented by the Royal Netherlands Academy of Arts and Sciences (KNAW) have been taken into account and secured as presented in Table 1.49

Although scientific integrity was assured as much as possible, we did face some hurdles in the publication of our results that are important to address. With every study submission, we have been completely transparent about the research being industry-funded. Consequently, several reviewers raised their concerns about possible conflicts arising from the industry funding, and some studies have been rejected (at least partly) based on these concerns. These experiences are important to mention because of two possible negative consequences for science. First, publication bias increases if credible and reliable, yet industry-funded studies are not published in scientific literature due to the reluctance of journals with regard to industry-funded research. For example, the study protocol presented in Chapter 4 was not accepted for publication because it was industry-funded. Consequently, we could have been selective in reporting outcomes in the particular RCT (Chapter 5), which would have had a counterproductive effect on scientific integrity. However, to avoid publication bias, the protocol was published online before commencement of the RCT. Second, reviewer concerns could give scientists perverse incentives to abstain from disclosing industry funding. Both potential consequences cause a threat to transparency and 


\section{Chapter 7}

hence to scientific integrity. Of course, potential conflicts of interests must be reported but should not be a reason per se for rejecting a study.

Table $1-$ KNAW principles

\begin{tabular}{|c|c|c|}
\hline $\begin{array}{l}\text { Scientific } \\
\text { KNAW } \\
\text { principles }\end{array}$ & $\begin{array}{l}\text { Explanation of } \\
\text { principle }\end{array}$ & Secured by: \\
\hline Honesty & $\begin{array}{l}\text { Honest reporting of } \\
\text { the research } \\
\text { process and } \\
\text { outcomes. }\end{array}$ & 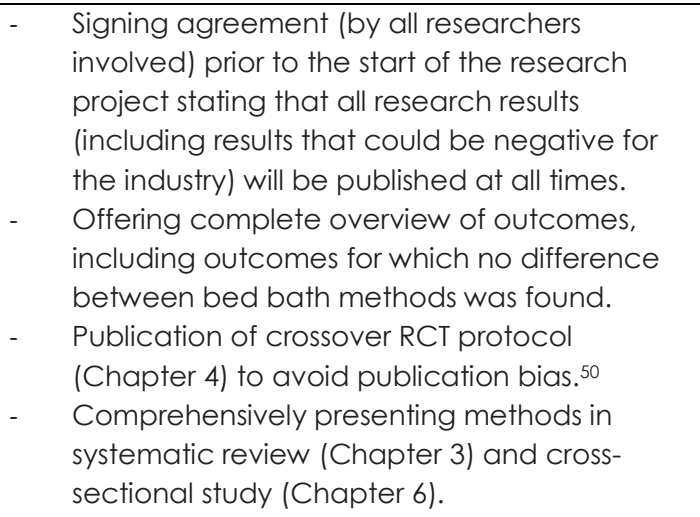 \\
\hline Scrupulousness & $\begin{array}{l}\text { Adopting scientific } \\
\text { methods in the } \\
\text { design, execution } \\
\text { and reporting } \\
\text { stages. }\end{array}$ & $\begin{array}{l}\text { Involving scientific experts, for example, in } \\
\text { data analysis of the crossover RCT (Chapter 5) } \\
\text { and in the development of the Bed Bath } \\
\text { Beliefs tool (Chapter 6). }\end{array}$ \\
\hline Transparency & $\begin{array}{l}\text { Transparent } \\
\text { presentation of the } \\
\text { research process } \\
\text { and results. }\end{array}$ & $\begin{array}{l}\text { - Offering complete overview of outcomes, } \\
\text { including outcomes for which no difference } \\
\text { between bed bath methods was found. } \\
\text { - Publication of crossover RCT protocol } \\
\text { (Chapter 4) to avoid publication bias. } 50 \\
\text { - Comprehensively presenting methods in } \\
\text { systematic review (Chapter 3) and cross- } \\
\text { sectional study (Chapter 6). }\end{array}$ \\
\hline Independence & $\begin{array}{l}\text { Research should not } \\
\text { be guided by non- } \\
\text { scientific (e.g. } \\
\text { commercial) } \\
\text { considerations. }\end{array}$ & $\begin{array}{l}\text { - Scientific supervision by scientists without any } \\
\text { stake in washing without water. }\end{array}$ \\
\hline Responsibility & $\begin{array}{l}\text { Taking into account } \\
\text { legitimate interests } \\
\text { of study participants } \\
\text { by conducting } \\
\text { relevant research. }\end{array}$ & $\begin{array}{l}\text { - Assessment and approval of relevant studies } \\
\text { by medical ethics committees. } \\
\text { Undertaking pilot interviews (for qualitative } \\
\text { study in Chapter 2) and pilot trial (for } \\
\text { crossover RCT in Chapter 5) to avoid harm to } \\
\text { participants. }\end{array}$ \\
\hline
\end{tabular}


In contrast to the hurdles we had to jump, industry funding also offered some advantages. For example, the industry sponsor allowed us the freedom to determine study objectives based on the results of preceding studies instead of a predetermined project proposal.51 For example, comfort perceptions have been studied in the crossover RCT (Chapter 5) based on the findings of the systematic review (Chapter 3) that showed a lack of evidence on patient experiences with the washing without water bed bath. To conclude, scientific integrity is secured because all parties, including the industry funder, are aware of the negative impact of deviant research conduct on the public's trust in science..$^{52}$ If public trust in science is harmed, no single party benefits.

\section{Validity - triangulation}

No single research, industry-funded or not, is free of bias that can possibly affect the internal validity. 53,54 This dissertation's internal validity increased by the application of several forms of triangulation. Triangulation is the application of different perspectives (e.g. in theory, methods, and of different study subjects) to get a better grasp of the truth, to obtain a more comprehensive understanding of the phenomenon under study, and to increase validity. ${ }^{55}$ The three types of triangulation applied in this dissertation are method, data, and investigator triangulation.

First, we applied method triangulation by conducting studies with varying designs to study the bed bath. Our designs include a systematic review, a qualitative study, and quantitative studies (i.e. a crossover RCT and a cross-sectional study). The combination of qualitative research (representing words) and quantitative research (representing numbers) especially contributes to the validity of the results, because qualitative findings can clarify and offer context to quantitative findings. ${ }^{55}$ For example, nurses mentioned doubts about the hygienic effectiveness of the washing without water bed bath in the qualitative study (Chapter 2), which might explain the slight preference for the water and soap bed bath among students being bathed in bed in our crossover RCT (Chapter 5). Furthermore, the qualitative findings were used to develop the Bed Bath Beliefs tool. Overall, the wide range of study methods applied in this dissertation led to a more complete picture of the bed bath and the use of washing without water.

Second, one of the main strengths of this dissertation is that we applied person triangulation (i.e. a type of data triangulation) by collecting data from many different actors, including nursing home residents (Chapter 2), nurses (Chapter 2 and 6), family members (Chapter 2), and nursing students (Chapter 5 and 6). ${ }^{55}$ By combining the perspectives of different health care actors, we increased the validity of our research findings with respect to actor experiences with the bed bath. Experiences are holistic and dependent on interrelationships between different actors. Therefore, both nursing literature (e.g. about relationship-centered care) ${ }^{9}$ and service literature advocate holistic research approaches that address high levels of complexity. ${ }^{56-58}$ The validity of our main findings about the importance of the bed bath and the suitability of the use of washing without water was enhanced because we 


\section{Chapter 7}

incorporated the perspectives of patients, nurses, family, and nursing students. Finally, we explored the bed bath from different "person levels", being the individual actor level and the collective actor-network level in our qualitative study (Chapter 2).

Third, we applied investigator triangulation throughout the research process. Investigator triangulation involves the use of complementary skills, knowledge, and divergent perspectives of multiple researchers to reduce biased decisions. ${ }^{55}$ At the operational level, we applied investigator triangulation by involving several researchers in research tasks and research decisions. For example, several researchers were involved in the data coding and interpretation stages of the qualitative study (Chapter 2), and experts in questionnaire design were involved in the scale development of the Bed Bath Beliefs tool (Chapter 6). At a more theoretical level, the multidisciplinary setup added to investigator triangulation. During the conduct of the research, monthly meetings with the multidisciplinary research team took place in which several theoretical interpretations and ideas were shared as input for this dissertation. Consequently, idiosyncratic bias was minimized. ${ }^{55}$

Although triangulation added to the validity of our findings, we shortly want to address two issues that could have affected the validity of our findings. First, it was striking to notice that validated measurement instruments were lacking for the physical comfort outcomes of our crossover RCT (Chapter 5). To measure physical comfort across patients, we used a single-item question. Patient physical comfort might have been measured more accurately if a multi-item scale was available for this construct. Furthermore, we used the physical demands scale to measure physical comfort across nurses. Because physical demands and physical comfort are related but not completely the same, our results might have been different if a validated scale for physical comfort could have been used.

The second issue we want to address is that we used nursing students as proxy respondents for real bedridden patients to measure the comfort perceptions of patients (Chapter 5). The use of proxy respondents allowed a highly controlled setup, which increased the accuracy of our results, but of course also has consequences for the generalizability of the findings. 59 Because the beliefs of nursing students can influence their perceptions about patients' perceptions and preferences, comfort perceptions and bed bath method preferences might differ between nursing students and actual bedridden patients.60, 61 Because generalizability never was a specific goal in this dissertation, future research on the bed bath and on washing without water could aim to include real bedridden patients. 


\section{FUTURE DIRECTIONS}

The results of this dissertation have relevant implications for health care practice. Furthermore, recommendations for future research can be made.

\section{Implications for health care practice}

The most important implications for health care practice directly relate to the two main findings of this dissertation, being the importance of the bed bath in general and the suitability of the washing without water intervention.

With respect to the bed bath in general, our results underline its importance as an essential of care, which stands in contrast to the undervaluation of essential care by many nurses worldwide. The experiences that were shared in our qualitative study (Chapter 2) inform health care managers and nurses that it is unacceptable to omit bed baths, which unfortunately happens sometimes in health care practice. 39,62 Health care managers should warrant that the bed bath gets enough attention by raising awareness that the bed bath is too important to omit or to be executed routinely by merely lower skilled nurses.62,63 The Bed Bath Beliefs tool (Chapter 6) could be used by health care managers to assess nurses' beliefs about the importance of the bed bath. Based on the scores of the Bed Bath Importance Belief scale, health care managers can take actions directed at beliefs that indicate a low perceived importance of the bed bath. This is important because nurses should especially recognize the importance of the bed bath. Our qualitative results (Chapter 2) show that the bed bath contributes to patient well-being if their needs for autonomy, competence, and relatedness are fulfilled. If nurses recognize the importance of the bed bath with respect to these needs and patient well-being, nurses are likely to feel more competent after providing a bed bath, which also adds to their own well-being.

Our results show washing without water is a suitable alternative to the traditional bed bath, and it can be implemented more widely in health care practice without deteriorating quality of care. Therefore, nurses should be open towards the use of washing without water and should be encouraged by health care managers who should advocate the use of washing without water in their health care institutions. Ideally, nurses should always be able and willing to offer patients a choice for the type of bed bath to support the autonomy and well-being of patients.33, 64 To enable nurses, health care management should provide the freedom to adapt the bed bath to the needs of the patient. This means, for example, that nurses should have the resources (e.g. different bed bathing materials) available to offer a choice. To increase the willingness of nurses to offer a choice to patients, health care managers must pay attention to possible unjustified negative preconceptions in nurses about the use of washing without water. Several nurses in our qualitative study (Chapter 2) indicated a preference for the traditional bed bath, which sometimes even deviated from particular patients' preferences but existed due to doubts about the effectiveness of washing without water. Health care managers could use the Bed Bath Beliefs tool (Chapter 6) to measure nurses' beliefs about the washing without water intervention. Consequently, they could inform nursing staff about the results of 


\section{Chapter 7}

this dissertation to show that the washing without water bed bath is at least as suitable as the traditional bed bath and to address possible unfavorable beliefs towards the use of washing without water.

Finally, our results have implications for nursing educators who play an important part in shaping nursing students', and therefore, future nursing professionals' beliefs about the bed bath. Nursing educators should rearrange their curricula to propagate the general importance of the bed bath. We were surprised to find out that practicing bed baths on real people was not (or no longer) part of nursing curricula of the Dutch nursing schools that participated in our crossover RCT (Chapter 5). Because nursing students are educated to provide bed baths to real patients, we think nursing students would benefit from personally experiencing what patients go through when they receive a bed bath. Therefore, nursing educators are encouraged to reintroduce practicing bed baths on fellow students. By doing so, nursing students are taught that the bed bath is too important to only cover in textbooks or in exercises on dummies. Moreover, if both bed bath methods are incorporated in the curricula, nursing students benefit from personally experiencing both the traditional bed bath and the washing without water bed bath. Consequently, nurses can make better informed decisions about the use of different bed bath methods and decisions might be based less on nurses' beliefs and assumptions. Furthermore, the appropriate use of washing without water is likely to improve if students gained personal experience with this bed bath method.

\section{Recommendations for future research}

Our results underline the scientific plea for more attention and for a revaluation of the bed bath as an essential of care. 65 Future research could contribute to the uplift of the importance of the bed bath. Based on our methodological reflection, we would like to make recommendations with respect to real patient trials and the development of reliable and validated measurement instruments. Furthermore, we present one recommendation with respect to the implementation of washing without water.

The first recommendation for future research is to measure the effects of the washing without water bed bath on the experiences of real bedridden patients. Although the amount of (international) evidence on washing without water increased after the publication of our systematic review (Chapter 3) (i.e. between 2017 and 2020 new studies have been conducted in Indonesia, Japan, Taiwan, the US, Canada, Brazil, Turkey, Denmark, and the Faroe Islands), 32, 66-75 the number of clinical trials that are conducted in actual health care settings is still low. Therefore, evidence on patient experiences with washing without water is still scarce. We did not study the experiences of real patients (e.g. with respect to comfort), because this would have been highly challenging. For example, washing without water is relatively widely adopted in the Netherlands, which made it difficult to execute a "clean" trial in which all participants would only receive the intervention or the control bed bath or in which participants did not already experience the washing without water bed bath. 
Furthermore, we chose a simulated laboratory setting (instead of a trial in health care practice) to control for many variables that would be present in health care practice and that could affect comfort experiences (e.g. interruptions during the bed bath). However, because of the need for evidence on the experiences of real patients, we encourage future researchers to study the effect of washing without water in actual health care settings with different "patient" populations, such as nursing homes, hospitals and home care.

To study effects of the use of washing without water, reliable and validated measurement instruments are required. As we commented earlier, it was difficult to find measurement instruments for the physical comfort outcomes of the bed bath. Therefore, we recommend future research develop measurement instruments that can be used in clinical trials on essentials of care, such as the bed bath. For example, instruments can be developed to measure the physical comfort of patients during essential health care activities. Current comfort scales focus heavily on pain but ignore other physical comfort dimensions, such as physical inconveniences and exhaustion. Apart from measurement instruments that can be applied to essential care in general, measurement instruments that are specifically relevant for the bed bath need to be developed. For example, instruments could be developed to measure patients' perceptions of being clean and fresh after a bed bath. This could be important because most doubts about the washing without water bed bath expressed in the qualitative study (Chapter 2) and in nurses' beliefs (Chapter 6) were related to perceived freshness after the washing without water bed bath.

Finally, future research could explore how washing without water might be best implemented in different health care segments, such as nursing homes. As we discussed in this chapter, washing without water could be included in the choice set for patients. However, to support patient autonomy, patients should still be able to also choose a traditional bed bath. Because nurses will likely face increasing time constraints, it might not be possible on some occasions to provide a traditional bed bath if patients would prefer that. Therefore, future research could study whether combinations of using both bed bath methods could be a solution, and if so, which combinations might be best based on nursing standards and patient preferences. For example, it might be best to provide patients a washing without water bed bath five days a week, once a week with water and soap, and once a week no bath at all. Furthermore, future studies could explore if washing without water could add to patients' self-reliance and independence, which could have consequences for its implementation. If washing without water enables patients to bathe themselves occasionally, patients could experience more autonomy with respect to the bed bath and nurses could be unburdened. 


\section{Chapter 7}

\section{CONCLUSION}

Experiences during and after the bed bath can influence the fulfillment of autonomy, competence and relatedness of patients, nurses and family members. Consequently, the bed bath is an essential care activity that contributes to the well-being of these actors. Washing without water was found to be a suitable bed bath method and an appropriate alternative to the traditional bed bath with water and soap. None of the findings showed an inferior performance of the washing without water bed bath compared to the traditional bed bath. Moreover, washing without water turns out to be shorter and less physically straining for nurses. Nurses also showed a preference for the washing without water bed bath from the nurse's perspective. However, nurses and nursing students indicated a preference for the water and soap bed bath from the patient's perspective. These preferences for the traditional bed bath cannot be explained by our results and therefore future research should further explore nurses' and (real) patients' experiences with the bed bath. 


\section{References}

1. Morillo F, Bordons M and Gómez I. Interdisciplinarity in Science: A Tentative Typology of Disciplines and Research Areas. Journal of the American Society for Information Science and Technology 2003;54:1237-1249.

2. Karlqvist A. Going beyond Disciplines: The Meanings of Interdisciplinarity. Policy Sciences 1999;32:379-383.

3. Madni AM. Transdisciplinarity: reaching beyond disciplines to find connections. Journal of Integrated Design and Process Science 2007;11:1-11.

4. de Chavez AC, Backett-Milburn K, Parry O, et al. Understanding and researching wellbeing: Its usage in different disciplines and potential for health research and health promotion. Health Education Journal 2005;64:70-87.

5. Weaver $\mathrm{K}$ and Olson JK. Understanding paradigms used for nursing research. Journal of Advanced Nursing 2006;53:459-469.

6. Rosenbaum MS. Transformative service research: focus on well-being. The Service Industries Journal 2015;35:363-367.

7. Sweeney JC, Danaher TS and MCColl-Kennedy JR. Customer Effort in Value Cocreation: Improving Quality of Life and Behavioral Intentions of Health Care Customers. Journal of Service Research 2015;18:318-335.

8. Pinho N, Beirão G, Patrício L, et al. Understanding value co-creation in complex services with many actors. Journal of Service Management 201 4;25:470-493.

9. Sion KYJ, Haex R, Verbeek H, et al. Experienced Quality of Post-Acute and LongTerm Care From the Care Recipient's Perspective-A Conceptual Framework. Journal of the American Medical Directors Association 2019:20:1386-1390.

10. Hoeck B and Delmar C. Theoretical development in the context of nursing - The hidden epistemology of nursing theory. Nursing Philosophy 2018;19.

11. McColl-Kennedy JR. Health care in service science special issue of Australasian Marketing Journal. Australasian Marketing Journal 2014;22:165-167.

12. Gunther M and Alligood MR. A discipline-specific determination of high quality nursing care. Journal of Advanced Nursing 2002;38:353-359.

13. Sarvimäki A and Stenbock-Hult B. Quality of life in old age described as a sense of well-being, meaning and value. Journal of Advanced Nursing 2000;32:1025-1033.

14. Grönroos C and Voima P. Critical service logic: making sense of value creation and co-creation. Journal of Academy of Marketing Science 2013;41:133-150.

15. Golinelli GM, Barile S, Saviano M, et al. Perspective Shifts in Marketing: Toward a Paradigm Change? Service Science 2012;4:121-134.

16. Gummesson E. Exit Services Marketing - Enter Service Marketing. The Journal of Customer Behaviour 2007;6:113-141.

17. Crossing the Quality Chasm: A new health system for the 21st century. Institute of Medicine. 2001. Available from: https://iom.nationalacademies.org/ /media/Files/Report\%20Files/2001/Crossingthe-Quality-Chasm/Quality\%20Chasm\%202001\%20\%20report\%20brief.pdf. Accessed 26 Nov 2015.

18. Klassen AC, Creswell J, Clark VLP, et al. Best practices in mixed methods for quality of life research. Quality of Life Research 2012;21:377-380.

19. Evers A, Jensen L and Paul H. Grensverleggend. Kansen en belemmeringen voor interdisciplinair onderzoek. De Jonge Akademie; 2015. 
20. Ryan RM and Deci EL. Self-Determination Theory and the Facilitation of Intrinsic Motivation, Social Development, and Well-Being. American Psychologist 2000;55:68-78.

21. Liehr P and Smith MJ. Middle Range Theory: A Perspective on Development and Use. Advances in Nursing Science 2017;40:51-63.

22. Cody WK. Middle-Range Theories: Do They Foster the Development of Nursing Science? Nursing Science Quarterly 1999;12:9-14.

23. Im EO and Chang SJ. Current Trends in Nursing Theories. Journal of Nursing Scholarship 2012;44:156-164.

24. Mudd A, Feo R, Conroy T, et al. Where and how does fundamental care fit within seminal nursing theories: A narrative review and synthesis of key nursing concepts. Journal of Clinical Nursing 2020;29:3652-3666.

25. Henderson $\vee$. The concept of nursing. Journal of Advanced Nursing 1978:3:113130.

26. Ryan RM and Deci EL. Intrinsic and Extrinsic Motivations: Classic Definitions and New Directions. Contemporary Educational Psychology 2000;25:54-67.

27. Quero MJ and Ventura R. The role of balanced centricity in the Spanish creative industries adopting a crowd-funding organisational model. Journal of Service Theory and Practice 2015;25:122-139.

28. Verleye $K$, Jaakkola E, Hodgkinson IR, et al. What causes imbalance in complex service networks? Evidence from a public health service. Journal of Service Management 2017:28:34-56.

29. Deci EL and Ryan RM. The "What" and "Why" of Goal Pursuits: Human Needs and the Self-Determination of Behavior. Psychological Inquiry 2000;1 1:227-268.

30. Sharma S, Conduit J and Hill SR. Hedonic and eudaimonic well-being outcomes from co-creation roles: a study of vulnerable customers. Journal of Services Marketing 2017:31:397-411.

31. Sherwin S and Winsby M. A relational perspective on autonomy for older adults residing in nursing homes. Health Expectations 2010;14:182-190.

32. Veje PL, Chen M, Jensen CS, et al. Bed bath with soap and water or disposable wet wipes: Patients' experiences and preferences. Journal of Clinical Nursing 2019;28:2235-2244.

33. Simmons SF, Rahman A, Beuscher L, et al. Resident-Directed Long-Term Care: Staff Provision of Choice During Morning Care. The Gerontologist 201 1:51:867-875.

34. Zwakhalen S, Metzelthin S, Groven F, et al. Samenwerking in Academische netwerken - Wassen zonder water. Innoveren in de dagelijkse pratkijk. Tijdschrift voor Ouderengeneeskunde 2017;6.

35. Kang B, Scales K, McConnell ES, et al. Nursing home residents' perspectives on their social relationships. Journal of Clinical Nursing 2020;29:1 162-1174.

36. McCormack B. Autonomy and the relationship between nurses and older people. Ageing and Society 2001;21:417-446.

37. Williams GC, Deci EL and Ryan RM. Building health-care partnerships by supporting autonomy: Promoting maintained behavior change and positive health outcomes. Partnerships in healthcare: Transforming relational process 1998:67-87.

38. Moloney W, Boxall $\mathrm{P}$, Parsons $M$, et al. Factors predicting Registered Nurses' intentions to leave their organization and profession: A job demands-resources framework. Journal of Advanced Nursing 2018;74:864-875. 
39. Parr JM, Bell J and Koziol-McLain J. Evaluating fundamentals of care: The development of a unit-level quality measurement and improvement programme. Journal of Clinical Nursing 2018;27:2360-2372.

40. Pipe TB, Connolly T, Spahr N, et al. Bringing Back the Basics of Nursing: defining patient care essentials. Nursing Admininstration Quarterly 2012;36:225-233.

41. Feo R, Kitson A and Conroy T. How fundamental aspects of nursing care are defined in the literature: A scoping review. Journal of Clinical Nursing 2018;27:21892229.

42. Baumeister RF and Leary MR. The need to belong: desire for interpersonal attachments as a fundamental human motivation. Psychological Bulletin 1995; 1 17:497-529.

43. Mallidou AA, Cummings GG, Schalm C, et al. Health care aides use of time in a residential long-term care unit: A time and motion study. International Journal of Nursing studies 2013;50:1229-1239.

44. Skarli JB. Creating or Destructing Value in Use? Handling Cognitive Impairments in Co-Creation with Serious and Chronically III Users. Administrative Sciences 2021;1 1.

45. Rader J, Barrick AL, Hoeffer B, et al. The bathing of older adults with dementia: easing the unnecessarily unpleasant aspects of assisted bathing. American Journal of Nursing 2006;106:40-48.

46. Wolf JA, Niederhauser V, Marshburn D, et al. Reexamining "Defining Patient Experience": The human experience in healthcare. Patient Experience Journal 2021;8:16-29.

47. Schwarz RP. Maintaining Integrity and Credibility in Industry-Sponsored Clinical Research. Controlled Clinical Trials 1991;12:753-760.

48. Watt D. On Becoming a Qualitative Researcher: The Value of Reflexivity. The Qualitative Report 2007;12:82-101.

49. Netherlands Code of Conduct for Research Integrity. KNAW, NFU, and NOW. 2018. Available

from:

https://www.vsnu.nl/files/documents/Netherlands\%20Code\%20of\%20Conduct\%2 Ofor\%20Research\%20Integrity\%202018.pdf. Accessed 16 Apr 2021.

50. Roumen FJ. Industry-Sponsored Research: How to Eliminate Bias? The European Journal of Contraception \& Reproductive Health Care 2015;20:155-157.

51. Edwards MA and Roy S. Academic Research in the 21 st Century: Maintaining Scientific Integrity in a Climate of Perverse Incentives and Hypercompetition. Environmental Engineering Science 2017;34:51-61.

52. Goodman JL and Borio L. Finding Effective Treatments for COVID-19 - Scientific Integrity and Public Confidence in a Time of Crisis. Journal of the American Medical Association 2020;323:1899-1900.

53. Rowe S, Alexander N, Clydesdale $F$, et al. Funding food science and nutrition research: financial conflicts and scientific integrity. Nutrition Reviews 2009;67:264272.

54. Smith J and Noble H. Bias in research. Evidence-Based Nursing 2014;17:100-101.

55. Polit DF and Beck CT. Nursing research: Generating and assessing evidence for nursing practice, 8th ed. Lippincott Williams \& Wilkins; 2008.

56. Black HG and Gallan AS. Transformative service networks: cocreated value as well-being. The Service Industries Journal 2015;35:826-845.

57. Osei-Frimpong K. Patient participatory behaviours in healthcare service delivery. Journal of Service Theory and Practice 2017;27:453-474. 
58. Gummesson E, Kuusela $H$ and Närvänen E. Reinventing marketing strategy by recasting supplier/customer roles. Journal of Service Management 2014;25:228240.

59. Pannucci CJ and Wilkins EG. Identifying and Avoiding Bias in Research. Plastic and Reconstructive Surgery 2010;126:619-625.

60. Neumann PJ, Araki SS and Gutterman EM. The Use of Proxy Respondents in Studies of Older Adults: Lessons, Challenges, and Opportunities. Journal of the American Geriatrics Society 2000;48:1646-1654.

61. Mattimore TJ, Wenger NS, Desbiens NA, et al. Surrogate and Physician Understanding of Patients' Preferences for Living Permanently in a Nursing Home. Journal of the American Geriatrics Society 1997:45:818-824.

62. Richards DA and Borglin G. 'Shitty Nursing' - the new normal? International Journal of Nursing Studies 2019:91:148-152.

63. Koehn ML and Lehman K. Nurses' perceptions of evidence-based nursing practice. Journal of Advanced Nursing 2008;62:209-215.

64. Liang HY, Chu CY and Lin JSC. Engaging customers with employees in service encounters: Linking employee and customer service engagement behaviors through relational energy and interaction cohesion. Journal of Service Management 2020;31:1071-1105.

65. Zwakhalen SMG, Hamers JPH, Metzelthin SF, et al. Basic nursing care: The most provided, the least evidence based. A discussion paper. Journal of Clinical Nursing 2018;27:2496-2505.

66. Nøddeskou LH, Túgvustein N, Marjunardóttir A, et al. Assessment of bed bathing methods in the Faroe Islands. The American Journal of Nursing Science 2018;7:109114.

67. Matsumoto C, Nanke K, Furumura S, et al. Effects of disposable bath and towel bath on the transition of resident skin bacteria, water content of the stratum corneum, and relaxation. American Journal of Infection Control 2019:47:81 1-815.

68. Hørdam B, Brandsen RV, Frandsen TK, et al. Nurse-assisted personal hygiene to older adults 65+ in home care setting. Journal of Nursing Education and Practice 2018;8:23-28.

69. Martin ET, Haider S, Palleschi M, et al. Bathing hospitalized dependent patients with prepackaged disposable washcloths instead of traditional bath basins: A casecrossover study. American Journal of Infection Control 2017:45:990-994.

70. Afelya $\mathrm{TI}$ and Arafat R. The effect of disposable bed bathing (DBB) on nurse satisfaction and the comfort of stroke patients in Wahidin Sudirohusodo Hospital. Indonesian Nursing Journal of Education and Clinic 2018:3:109-112.

71. Veje PL, Chen M, Jensen CS, et al. Effectiveness of two bed bath methods in removing microorganisms from hospitalized patients: A prospective randomized crossover study. American Journal of Infection Control 2020;48:638-643.

72. Büyükyılmaz $\mathrm{F}$ and Şendir M. Opinions of Intensive Care Nurses: Traditional or Disposable Wipes Bed Bath? A Quasi-Qualitative and Cost Analysis Study. International Journal of Nursing \& Clinical Practices 2017:4:1-5.

73. Toledo LV, Salgado PDO, Souza CCD, et al. Effects of dry and traditional bed bathing on respiratory parameters: a randomized pilot study. Revista LatinoAmericana Enfermagem 2020;28.

74. Tai CH, Hsieh TC and Lee RP. The Effect of Two Bed Bath Practices in Cost and Vital Signs of Critically III Patients. International Journal of Environmental Research and Public Health 2021;18. 
Chapter 7

75. Paulela DC, Bocchi SCM, Mondelli AL, et al. Effectiveness of bag bath on microbial load: clinical trial. Acta Paulista de Enfermagem 2018;31:7-16. 

SUMMARY 
This dissertation is about the bed bath and more specifically about the use of the washing without water intervention for this essential nursing activity. Washing without water are disposable prepacked products containing bathing cloth impregnated with a cleansing fluid, which can be used as an alternative to the traditional bed bath with water and soap. If washing without water is used, nurses do not need to use traditional bed bath materials, such as towels, wash basins or water. The aim of this dissertation was to assess the use of the washing without water intervention based on the experiences of health care actors. Experiences are important to consider, as these are related to actor well-being. Several studies were conducted with varying research designs. First, an explorative qualitative study was conducted to study the importance of the bed bath in general with respect to individual actor and collective actor network well-being (Chapter 2). Second, a systematic review was executed to get an overview of the available evidence on washing without water (Chapter 3). Third, a crossover randomized controlled trial was conducted to study the effect of washing without water on emotional and physical comfort (Chapter 4 and 5). Fourth, a crosssectional study was performed, which introduces and psychometrically tests the Bed Bath Beliefs tool (Chapter 6). The current chapter presents a summary of all the chapters of this dissertation.

Chapter 1 provides a general introduction to this dissertation. The bed bath is introduced from a service science and from a nursing science perspective, and the washing without water intervention is described. The general importance of the bed bath and the importance of autonomy, essentials of care and relationship-centered care are addressed. Finally, the aim of this dissertation is presented.

The general importance of the bed bath is explored in Chapter 2 based on actor (i.e. nursing home residents, nurses, and family members) experiences with the bed bath. The qualitative study of this chapter presents a conceptual model on balanced centricity as an indicator of network well-being. Balanced centricity is positioned as a situation in which the needs of all actors that are involved in a service (in this case, the bed bath) are fulfilled. Based on experiences of the bed bath from eight patients, six nurses, and five family members, fulfillment of actors' psychological needs of autonomy, competence and relatedness was found to contribute to individual actor well-being. If the psychological needs of all actors can be fulfilled simultaneously, balanced centricity emerges and therefore network well-being increases. However, if tensions between actors' needs exist, not all actors' psychological needs can be fulfilled by value co-creation, and network well-being is jeopardized.

The systematic review in Chapter 3 identified only six studies that compared the washing without water bed bath with the traditional bed bath for the complete body wash. Only two of these studies were assessed to be of high quality, which shows that the amount of high-quality evidence on washing without water is scarce. The available evidence showed that the washing without water bed bath performed 
equally well on some outcomes (e.g. hygienic effectiveness and resistance during the bed bath) and outperformed the traditional bed bath on other outcomes (e.g. occurrence skin abnormalities and bathing completeness).

An elaborate study protocol is presented in Chapter 4, which explains in greater detail how the crossover randomized controlled trial (RCT) presented in Chapter 5 has been conducted. In this crossover RCT, nursing students were randomly assigned the role of a patient (student patients) or the role of a nurse (student nurses). The "student patients" were bathed by the "student nurses" in a simulated laboratory setting. All students provided or received a traditional bed bath and a washing without water bed bath in a randomly assigned order. The results of this study showed that the washing without water bed bath is less physically demanding for nurses providing the bed bath and takes less time to complete. No difference was found with respect to emotional or physical comfort experienced by the students bathed in bed. Finally, although the grades assigned to the two bed bath methods did not differ, students that were bathed in bed had a slight preference for the traditional bed bath. Students that provided the bed bath preferred the washing without water bed bath, which also received a higher grade by these students.

Chapter 6 dives deeper into nurses' beliefs about the bed bath. The study in this chapter introduces and psychometrically tests the Bed Bath Beliefs tool that can be used to measure nurses' beliefs about the general importance of the bed bath and about the use of the washing without water intervention. Two promising scales were developed for the Bed Bath Beliefs tool. The Bed Bath Importance Beliefs scale measures nurses' beliefs about the general importance of the bed bath, and the Washing Without Water Beliefs scale measures nurses' beliefs about the use of washing without water. These beliefs are important, because beliefs can affect the execution of the bed bath and the proper use of the washing without water intervention. Although the data primarily is used to develop and test the scale, the data also reveals that nurses generally attach a fairly high importance to the bed bath and that nurses show mostly favorable beliefs about the use of washing without water.

The main findings and implications of this dissertation's results are discussed in Chapter 7. A theoretical discussion is provided about our multidisciplinary research setup, about self-determination theory as a theoretical context for the bed bath, and about the findings regarding washing without water (Chapter 3-6) with respect to the fulfillment of actors' psychological needs (central to Chapter 2). Furthermore, methodological considerations regarding scientific integrity and validity are discussed. Finally, future directions for healthcare practice and scientific research are proposed. 



\section{SAMENVATING}


Dit proefschrift gaat over de lichaamswassing op bed en in het bijzonder over het gebruik van de wassen-zonder-water interventie voor deze essentiële verpleegkundige handeling. Wassen-zonder-water omvat wegwerpbare pakketjes met wasdoekjes die geïmpregneerd zijn met een reinigende lotion. De wassenzonder-water interventie kan worden gebruikt als een alternatief voor de traditionele lichaamswassing op bed met water en zeep. Bij het gebruik van wassen-zonder-water hoeven verpleegkundigen niet langer gebruik te maken van traditionele wasbenodigdheden zoals handdoeken, waskommen of water. Het doel van dit proefschrift was het gebruik van de wassen-zonder-water interventie te beoordelen op basis van ervaringen van actoren in de zorg. Ervaringen staan centraal in onze beoordeling omdat ervaringen belangrijk zijn met betrekking tot het welzijn van actoren. Verschillende studies zijn uitgevoerd met elk een andere onderzoeksopzet. Allereerst is een exploratief, kwalitatief onderzoek gedaan naar het algemene belang van de lichaamswassing op bed voor het welzijn van individuele actoren en het welzijn van actor-netwerken (hoofdstuk 2). Ten tweede is een systematische review vitgevoerd om een overzicht te krijgen van de beschikbare bewijslast voor wassenzonder-water (hoofdstuk 3). Ten derde wordt het effect van het gebruik van wassenzonder-water op emotioneel en fysiek comfort onderzocht in een crossover gerandomiseerd gecontroleerd experiment (hoofdstuk 4 en 5). Ten vierde wordt de "Bed Bath Beliefs tool" geïntroduceerd en psychometrisch getest in een crosssectionele studie (hoofdstuk 6). Dit hoofdstuk presenteert een samenvatting van alle hoofdstukken uit dit proefschrift.

De algemene introductie van dit proefschrift wordt gepresenteerd in hoofdstuk 1. De lichaamswassing op bed wordt geïntroduceerd vanuit een service wetenschappelijk en vanuit een verpleegkundig wetenschappelijk perspectief. Daarnaast wordt de wassen-zonder-water interventie beschreven. Het algehele belang van de lichaamswassing op bed alsook het belang van autonomie, essentiële zorg, en "relationship-centered care" (relatie-gecentreerde zorg) worden behandeld. Tot slot wordt de doelstelling van het proefschrift gepresenteerd.

In hoofdstuk 2 wordt het algehele belang van de lichaamswassing op bed onderzocht op basis van ervaringen met de lichaamswassing van verschillende actoren (verpleeghuisbewoners, verpleegkundigen en familieleden). In deze kwalitatieve studie wordt een conceptueel model gepresenteerd over "balanced centricity" dat als indicator van netwerk-welzijn wordt gepositioneerd. Balanced centricity is een situatie waarin de behoeftes van alle actoren (personen) die betrokken zijn bij een dienst - in dit geval de lichaamswassing op bed - worden vervuld. Gebaseerd op ervaringen met de lichaamswassing op bed van acht verpleeghuisbewoners, zes verpleegkundigen en vijf familieleden, wordt geconcludeerd dat het welzijn van individuele actoren toeneemt als de psychologische behoeftes van deze actoren worden vervuld. De psychologische behoeftes zijn de behoefte voor autonomie, bevoegdheid en verbondenheid. 
Balanced centricity ontstaat als de psychologische behoeftes van alle betrokken actoren tegelijkertijd kunnen worden vervuld. Dan zal ook het welzijn van het gehele netwerk van actoren toenemen. Echter, als er spanningen bestaan tussen de behoeftes van actoren kunnen niet alle psychologische behoeftes van alle actoren tegelijkertijd worden vervuld en komt het welzijn van het netwerk in het geding.

Hoofdstuk $\mathbf{3}$ is een systematische review van studies waarin de wassen-zonder-water lichaamswassing wordt vergeleken met de traditionele, volledige lichaamswassing op bed. Slechts zes studies worden gepresenteerd, waarvan maar twee worden beoordeeld van hoge kwaliteit te zijn. Dit toont aan dat hoogkwalitatieve bewijslast voor de wassen-zonder-water interventie schaars is. Het beschikbare bewijs laat zien dat de lichaamswassing op bed met wassen-zonder-water met betrekking tot sommige vitkomsten even goed presteert (bijvoorbeeld op het gebied van hygiënische effectiviteit en weerstand tijdens de lichaamswassing op bed) en met betrekking tot andere vitkomsten beter presteert dan de traditionele lichaamswassing op bed (bijvoorbeeld met betrekking tot de huidconditie en compleetheid van de lichaamswassing).

Het protocol in hoofdstuk $\mathbf{4}$ beschrijft gedetailleerd hoe het crossover gerandomiseerde, gecontroleerde experiment (RCT) dat in hoofdstuk 5 wordt gepresenteerd, is uitgevoerd. Voor deze RCT-studie hebben studenten verpleegkunde willekeurig de rol van patiënt (student patiënten) of verpleegkundige (student verpleegkundigen) gekregen. De "student verpleegkundigen" hebben de "student patiënten" op bed gewassen in een gesimuleerde laboratorium setting. Alle deelnemende studenten hebben in een willekeurige volgorde een lichaamswassing op bed met wassen-zonder-water en een traditionele lichaamswassing op bed gekregen of gegeven. De resultaten tonen aan dat de wassen-zonder-water lichaamswassing op bed minder fysiek belastend is voor de verpleegkundigen en dat deze lichaamswassing sneller klaar is dan de traditionele lichaamswassing op bed. Met betrekking tot het emotioneel en fysiek comfort van studenten die op bed gewassen zijn, is geen verschil gevonden tussen de twee was methodes. Tot slot hebben "student patiënten" een lichte voorkeur voor de traditionele lichaamswassing op bed, hoewel geen verschil zichtbaar is in de cijfers die deze studenten geven aan beide was methodes. "Student verpleegkundigen" hebben een voorkeur voor wassen-zonder-water en geven ook een hoger cijfer aan deze was methode.

In hoofdstuk 6 wordt aandacht besteed aan meningen van verpleegkundigen over de lichaamswassing op bed. De studie in dit hoofdstuk introduceert de "Bed Bath Beliefs tool" dat gebruikt kan worden om meningen van verpleegkundigen te meten over het algehele belang van de lichaamswassing op bed en over het gebruik van wassen-zonder-water. De tool wordt psychometrisch beoordeeld op basis van data van verpleegkundigen en studenten verpleegkunde. De tool bevat twee 
veelbelovende schalen: de "Bed Bath Importance Beliefs" schaal meet meningen van verpleegkundigen over het algehele belang van de lichaamswassing op bed en de "Washing Without Water Beliefs" schaal meet meningen van verpleegkundigen over het gebruik van wassen-zonder-water. Deze meningen kunnen belangrijk zijn voor de vitvoering van de lichaamswassing op bed en het goed gebruik van de wassen-zonder-water interventie. Hoewel de data primair is verzameld voor de ontwikkeling en het psychometrisch testen van de tool, laat de data ook zien dat de deelnemers de lichaamswassing belangrijk vinden en dat zij overwegend positieve meningen hebben over het gebruik van wassen-zonder-water.

De belangrijkste resultaten en implicaties van dit proefschrift worden behandeld in hoofdstuk 7. Een theoretische behandeling wordt gepresenteerd waarin: de multidisciplinaire onderzoeksopzet wordt behandeld; waarin self-determination theory als context voor de lichaamswassing op bed wordt gepositioneerd; en waarin de bevindingen met betrekking tot de wassen-zonder-water interventie (uit hoofdstuk 3-6) worden gerelateerd aan de vervulling van de psychologische behoeftes van de bij de lichaamswassing betrokken actoren (hoofdstuk 2). Daarnaast worden methodologische overwegingen met betrekking tot wetenschappelijke integriteit en validiteit behandeld. Tot slot doen we in dit hoofdstuk een voorstel voor toekomstig onderzoek en voor gebruik van onze resultaten in de gezondheidszorgpraktijk. 
In this dissertation's introduction, hypothetical narratives were presented for the patient Mr. Johnson, Nurse Ms. Jackson, and family member Mrs. Quinn. Mr. Johnson needs to be bathed in bed and finds personal hygiene important but experiences a lack of control over the bed bath. Nurse Jackson is described as facing high workloads, especially during morning care when multiple patients need to be bathed, often in bed. Finally, family member Quinn struggled with her dad's (Mr. Johnson) mood swings, which seemed to be partially influenced by his experiences with the bed bath and wondered how she could support her dad's well-being. For the course of this research, we have talked to nursing home residents (patients), nurses and family members whose experiences showed resemblances with the hypothetical narratives. Based on their experiences with the bed bath, one of the foremost conclusions of this dissertation is the general importance of the bed bath to the well-being of these actors. The other main conclusion of this dissertation is that the washing without water intervention is a suitable alternative to the traditional bed bath with water and soap.

In this chapter, we will present the societal and scientific impact of this dissertation. First this dissertation's societal impact is described by paying attention to the concept of customer delight, by relating our findings to time constraints in health care (amongst others during the COVID-19 pandemic), and by presenting implications for nursing education. Second, the presentation of the scientific impact will focus on the interdisciplinary research approach and our model on balanced centricity.

\section{SOCIETAL IMPACT}

\section{The bed bath as a delightful experience}

Customer delight is described as "a positive emotion that people experience in response to having their expectations exceeded to an unanticipated and surprising degree" (p. 2). " Actor well-being is positioned in this dissertation to be dependent on actor experiences; therefore, we posit that customer delight, as a positive experience, could contribute to actor well-being. In the case of the bed bath, customer delight mainly refers to delightful experiences of patients being bathed in bed. Although the bed bath might seem an activity in which delightful experiences are unlikely, several studies found that customer delight can also emerge in relatively mundane service encounters or even in times of crisis, such as the COVID-19 pandemic. ${ }^{2}$ Our results have societal impact by showing health care managers and nurses how the bed bath in general, and the use of washing without water could lead to customer delight.

We will focus on a few determinants of customer delight that are applicable to the bed bath and relevant for the use of washing without water, being autonomy, solving a customer problem, and sensory experiences. Experiences of having some control over the service experience (i.e. autonomy) contribute to customer delight.' Therefore, if patients experience control over or choice in the course of the bed bath (which relates to their need for autonomy), patients are more likely to feel delighted. 
Unfortunately, morning care, including the bed bath, often is focused on the taskoriented health care rhythm instead of on patient choices.3, 4 Health care managers and nurses can increase patients' autonomy (and therefore contribute to delight) by offering patients a choice between the traditional bed bath and the washing without water bed bath. Another determinant of customer delight is solving a customer problem or fulfilling a customer need. For the bed bath, patient delight can be created if the bed bath fulfills patients' needs, such as their need for hygiene or comfort. Our results indicate that washing without water is able to fulfill such patients' needs at least equally well as the traditional bed bath. Moreover, washing without water could add to customer delight because it outperforms the traditional bed bath on some outcomes, such as patients' skin condition. For example, one resident interviewed for our qualitative study mentioned that she loves the smooth feeling of her skin after a bed bath with washing without water. The "skin feeling" is an example of a sensory experience, which is another determinant of customer delight. Therefore, health care professionals can contribute to patient delight with the use of washing without water. Furthermore, the industry could contribute to patient delight by developing pleasant sensory experiences, for example, by using pleasant fragrances in washing without water products.

\section{The bed bath in times of time constraints in health care}

Nurses are increasingly facing high workloads, also during morning care, 5 which negatively affects their mental health. Mental health is described as "a state of wellbeing in which the individual realizes his or her own abilities, can cope with the normal stresses of life, can work productively and fruitfully and is able to make a contribution to his or her community" (p. 1336). ${ }^{6}$ The mental health of nurses is negatively affected by the increased workload, which causes stress, which in turn could lead to physical and psychological complaints, job dissatisfaction and even intentions to leave the profession. ${ }^{4,7}$ Moreover, the quality of care offered to patients could suffer because nurses that face time constraints and high workloads generally perform worse..$^{5}$ Our findings indicate that washing without water could decrease the burden on nurses (both physically and in terms of time) and thereby support the well-being of nurses and patients.

Therefore, health care managers should especially consider the use of washing without water in case of time constraints. The washing without water bed bath has been shown to be shorter than, yet at least as suitable as, the traditional bed bath. Moreover, nurses can feel assured that they live up to their responsibility of offering a proper bed bath if they provide a washing without water bed bath. We found no evidence for any underperformance of the washing without water bed bath across a range of outcome measures and patient experiences (e.g. patients' perceived emotional comfort during the bed bath). Furthermore, findings indicate that washing without water outperforms the traditional bed bath with respect to skin condition, time effectiveness, bathing completeness, and physical demands for nurses. 
Despite the workload on nursing staff and the growing evidence about the washing without water intervention, the traditional bed bath is still expected to be provided most. Even 20 years after its introduction, washing without water is seen as an innovation by many. Times of excessive burden on health care systems, such as the COVID-19 pandemic, could offer an opportunity to rearrange bathing routines and could (combined with the new evidence provided by this dissertation) be a catalyst for the use of the washing without water intervention.8,9 Also post-corona, the increasing demand for care and the decreasing availability of health care workers could urge health care institutions to implement innovations, such as washing without water, to alleviate nurses' stress levels and even retain nurses. 5,7

\section{Implications for nursing education}

This dissertation offers valuable insights for nursing educators. First of all, the results can be included in teaching materials about bed bathing methods, which currently predominantly focus on the traditional bed bath. Second, our research showed the added value for nursing students to acquire personal experience with providing the bed bath or receiving the bed bath as a patient. Although many students refused to participate in our crossover RCT, enough students were still willing to participate and some mentioned how worthwhile it was to personally experience the bed bath. By (re)introducing bed bathing exercises on real people in the curricula, this experience can be offered to more nursing students, including those that are too shy or insecure to voluntarily participate in a research experiment. Finally, nursing educators could consider to apply service marketing theories, such as balanced centricity or value cocreation, to their courses. Some nursing students might find additional intrinsic motivation if they have the possibility to look at their future profession from another perspective.

\section{SCIENTIFIC IMPACT}

Apart from the societal impact, we would like to discuss two scientific impacts: 1) the added value of interdisciplinary research and 2) our model on balanced centricity.

First, we would like to plea for more multidisciplinary or even interdisciplinary research. We have approached the bed bath from a multidisciplinary perspective. The research team consisted of scientists from nursing science and service (marketing) science and the discussion between the different disciplines in the discourse of the research led to interesting findings. Interdisciplinary research can contribute to scientific innovation and the generation of discipline-transcending knowledge, 10 which is also applicable to our general discussion in which we integrated the psychological needs of autonomy, competence and relatedness with the findings about the use of the washing without water intervention. The adoption of service marketing theories, such as value co-creation, has been proven fruitful and increased our impact on nursing science. 
The second scientific impact of our dissertation is the presentation of the balanced centricity model in Chapter 2, which adds to our understanding of network well-being. This conceptual model shows the importance of fulfilling the needs of all actors involved in value co-creation; in our specific case, the needs of patients, nurses and family members involved in the bed bath. The model shows that actors have different needs, which all relate to the universal psychological needs of autonomy (i.e. to have control), competence (i.e. to be proficient), and relatedness (i.e. to belong). If actors' needs are aligned, all actors' needs can be fulfilled and the wellbeing of all actors increases. However, sometimes actors' needs are conflicting and therefore not all actors' needs can be fulfilled by and during the bed bath. Consequently, actor well-being and network well-being is pressured. If all actors' needs are fulfilled, balanced centricity is achieved (i.e. a situation in which all actors' needs are fulfilled simultaneously), which we posit as an indicator for network wellbeing. Our model could be valuable to understand actor and system well-being in other health care and service activities, in other service systems, and for other kinds of actors.

\section{DISSEMINATION}

To ensure proper use of washing without water, it is important that all parties involved are informed about the evidence about this intervention. Because mainly health care managers decide on the use of washing without water, "1 this dissertation is a valuable source of information for them. Health care managers can share this dissertation's results with nursing staff and involve them in decisions with respect to the bed bath. Involving nurses in the decision-making process is stressed to be important for the use of innovations in health care. ${ }^{12}$

The findings are already disseminated in several ways. All studies have been submitted to academic journals, three of these studies are published as an open access study, and one study is available online. Furthermore, some of the studies were presented during international conferences, such as Servsig, the European Nursing Congress and the Gerontological Society of America. In addition to dissemination in scientific journals and conferences, results are presented at the website of basic care revisited and in the popular science magazine "Quest". The results have also been presented to health care managers of different health care institutions in the Netherlands who were interested in using the washing without water intervention or who participated in the research. Finally, the research is presented to the industry at a conference of the nonwoven branch organization EDANA.

We will continue to disseminate the findings of this research with health care institutions, nursing educators, policy makers, and researchers. If desired, the results can be presented and discussed personally with interested health care managers and nursing educators. Health care managers and nursing educators can use the Bed Bath Beliefs tool, which is fully presented in this dissertation (see Appendix 1 for the tool in Dutch). Also researchers can use and refine this tool and are invited to use our 
Impact

conceptual model on balanced centricity. Policy makers can use our findings as input for guidelines, for example, on daily care activities. Finally, the findings are shared with the industry (sponsor) who could use the information to identify innovation opportunities, which ultimately could benefit health care. 


\section{References}

1. Parasuraman A, Ball J, Aksoy L, et al. More than a feeling? Toward a theory of customer delight. Journal of Service Management 2020;32:1-26.

2. Barnes DC, Mesmer-Magnus J, Krallman A, et al. Customer delight during a crisis: understanding delight through the lens of transformative service research. Journal of Service Management 2020;32:129-141.

3. Sherwin S and Winsby M. A relational perspective on autonomy for older adults residing in nursing homes. Health Expectations 2010;14:182-190.

4. Mallidou AA, Cummings GG, Schalm C, et al. Health care aides use of time in a residential long-term care unit: A time and motion study. International Journal of Nursing studies 2013;50:1229-1239.

5. Jun J, Ojemeni MM, Kalamani R, et al. Relationship between nurse burnout, patient and organizational outcomes: systematic review. International Journal of Nursing Studies 2021;119.

6. Lorente L, Vera M and Peiró T. Nurses' stressors and psychological distress during the COVID-19 pandemic: The mediating role of coping and resilience. Journal of Advanced Nursing 2021;77:1335-1344.

7. Moloney W, Boxall P, Parsons $M$, et al. Factors predicting Registered Nurses' intentions to leave their organization and profession: A job demands-resources framework. Journal of Advanced Nursing 2018;74:864-875.

8. Heinonen K and Strandvik T. Reframing service innovation: COVID-19 as a catalyst for imposed service innovation. Journal of Service Management 2021;32:101-112.

9. Brodie RJ, Ranjan KR, Verreynne ML, et al. Coronavirus crisis and health care: learning from a service ecosystem perspective. Journal of Service Theory and Practice 2021;31:225-246.

10. van Rijnsoever FJ and Hessels LK. Factors associated with disciplinary and interdisciplinary research collaboration. Research Policy 201 1;40:463-472.

11. Zwakhalen S, Metzelthin S, Groven F, et al. Samenwerking in Academische netwerken - Wassen zonder water. Innoveren in de dagelijkse pratkijk. Tijdschrift voor Ouderengeneeskunde 2017;6.

12. Anvik C, Vedeler JS, Wegener $C$, et al. Practice-based learning and innovation in nursing homes. Journal of Workplace Learning 2020;32:122-134. 
Impact

\section{Appendix 1 - Bed Bath Beliefs Tool (in Dutch)}

Beste zorgverlener,

Dank $u$ wel dat $u$ mee wilt doen met het onderzoek naar de wasbeurt op bed van cliënten/bewoners/ patiënten (vanaf nu cliënten genoemd). Het invullen van de vragenlijst is anoniem. Uw antwoorden zijn alleen voor het onderzoeksteam toegankelijk en worden alleen voor onderzoeksdoeleinden gebruikt.

Probeer de vragenlijst a.u.b. volledig in te vullen. Eerst volgen hieronder enkele vragen over uzelf:

1. Wat is uw geslacht? Zet een kruisje voor uw antwoord.

Man

Vrouw

Anders, namelijk

2. Wat is uw leeftijd: . jaar

3. Wat is uw hoogstgenoten afgeronde opleiding? Zet een kruisje voor uw antwoord.

Zorghulp

Helpende

Verzorgende

Verzorgende (IG)

Verpleegkundige (MBO opgeleid)

Verpleegkundige (HBO opgeleid)

Anders, namelijk:

4. Wat is uw huidige functie? Zet een kruisje voor uw antwoord.

Zorghulp

Helpende

Verzorgende

Verzorgende (IG)

Verpleegkundige (MBO opgeleid)

Verpleegkundige (HBO opgeleid)

Anders, namelijk:

5. In welke zorgsector werkt u?

Ziekenhuis

Verpleeghuis

Thuiszorg

Anders, namelijk: 
6. Wat is het type afdeling waar $u$ werkt (afdeling waarvoor $u$ deze vragenlijst invult)? Zet een kruisje voor uw antwoord.

Somatische afdeling

Psychogeriatrische afdeling

Anders, namelijk:

7. Hoe lang bent $u$ al werkzaam (indien $u$ dit niet precies weet, probeer dan in te schatten):

a. In de ouderenzorg? maanden.

jaar en

b. Binnen uw huidige zorgorganisatie? maanden.

.jaar en

c. Op uw huidige afdeling?

jaar en maanden.

8. Ongeveer hoeveel uur per week verleent $u$ werkelijk gemiddeld zorg aan cliënten in deze organisatie (dit kan verschillen van het aantal uur in uW contract):

uur.

Vanaf hier gaat het bij alle vragen altijd over de volledige wasbeurt op bed van cliënten die ondersteuning nodig hebben. Wij bedoelen hiermee een lichaamswassing waarbij de zorgverlener alle lichaamsdelen van de cliënt op bed wast (eventueel met hulp van de cliënt). Doorgaans wordt dit in de ochtend gedaan met waskommen, water en zeep of met wegwerpbare natte doekjes of washandjes. Een volledige wasbeurt op bed is dus niet "alleen de onderbeurt" (incontinentiezorg) of "wasbeurten die niet op bed worden gegeven" (zoals bijvoorbeeld een wasbeurt aan de wasbak of in de douche).

9. In de tabel hieronder staan enkele stellingen over wat $u$ vindt van de volledige wasbeurt op bed. Per stelling kunt $u$ aangeven in hoeverre $u$ het ermee eens bent door naast elke stelling een kruisje te zetten in de kolom van uw antwoord. Er zijn geen goede of foute antwoorden, het gaat om uw mening over de volledige wasbeurt op bed. 


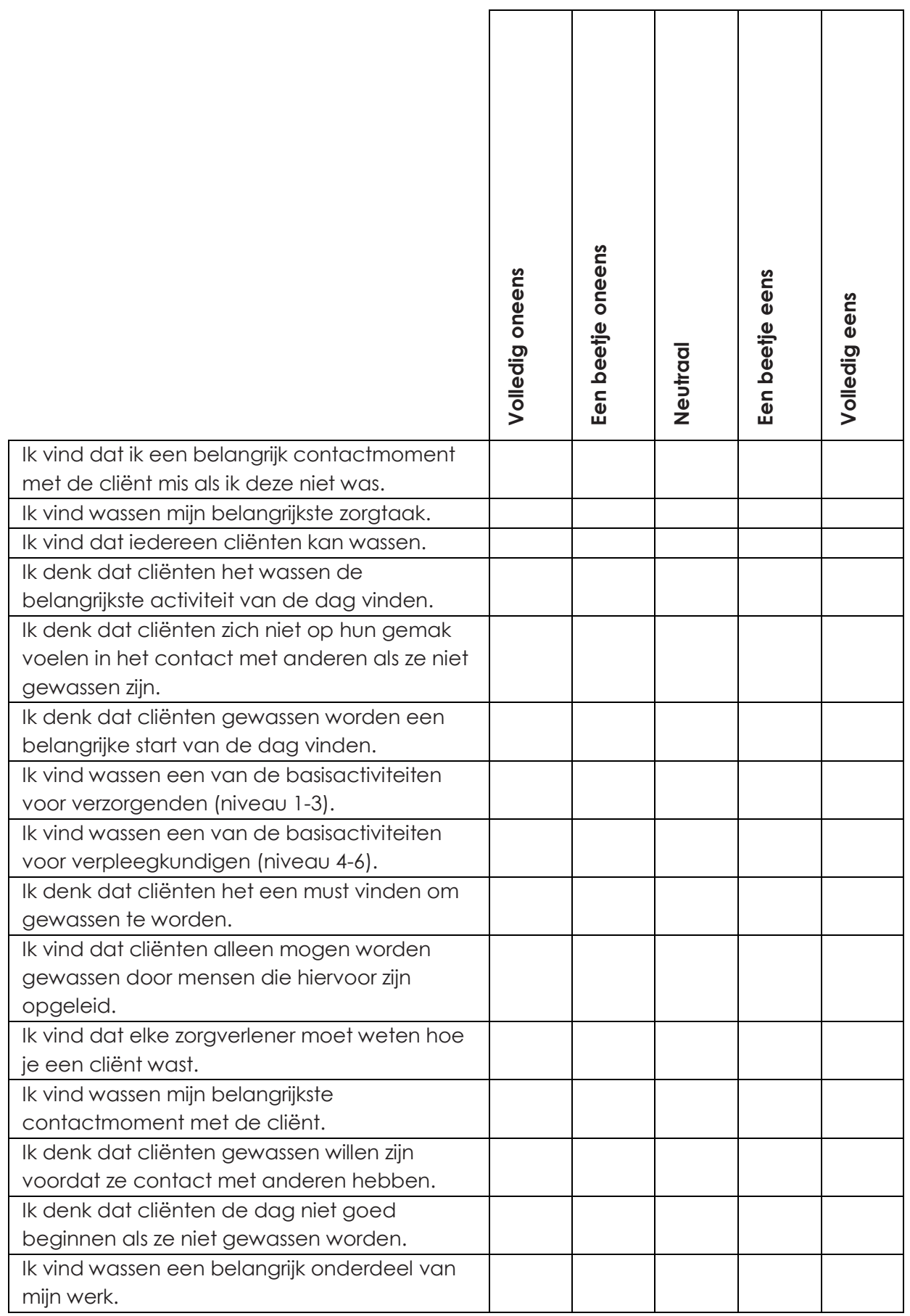




\section{Wassen-zonder-water}

Volledige wasbeurten op bed kunnen worden gegeven met water en zeep of met wassen-zonder-water. Dit zijn pakketjes met wegwerpbare wasdoekjes of washandjes met hierin een waslotion. Wasbakken, water, zeep, handdoeken en katoenen washandjes zijn dan niet meer nodig. Voorbeelden van wassen-zonderwater producten zijn hieronder afgebeeld. Vanaf nu gaan de vragen over wassenzonder-water.
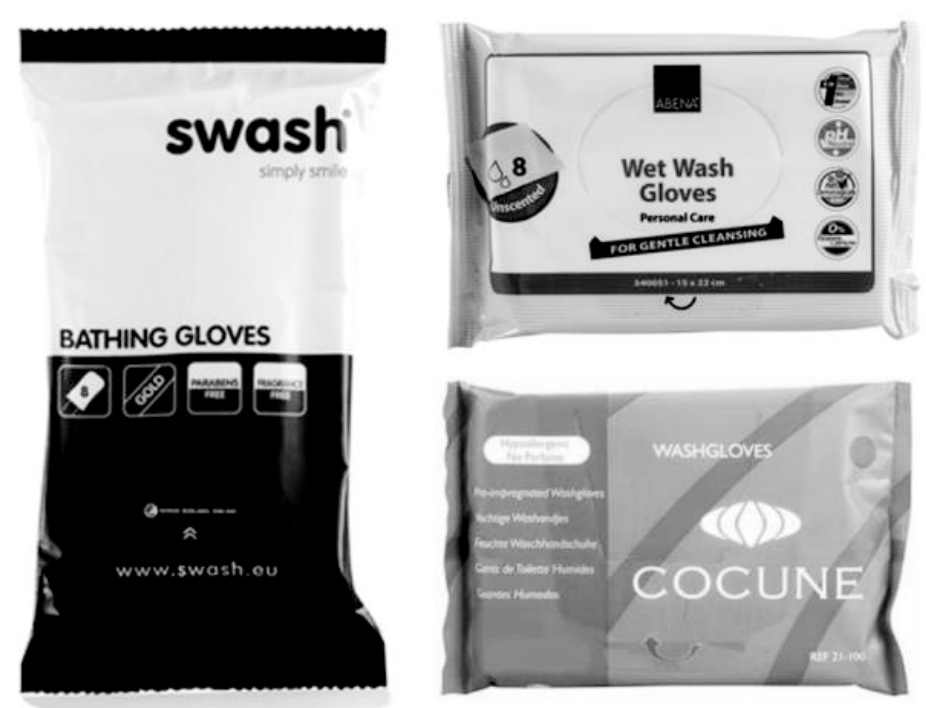

10. Wist $u$ voordat $u$ begon met het invullen van deze vragenlijst wat het wassen-zonder-water concept was?

Ja (ga door naar vraag 11)

Nee (ga door naar vraag 13)

11. Heeft u het wassen-zonder-water concept al eens gebruikt voor een volledige wasbeurt op bed van een cliënt?

Ja (ga door naar vraag 12)

Nee (ga door naar vraag 13)

12. Ongeveer hoe vaak per week gebruikt u momenteel wassen-zonder-water voor de volledige wasbeurt op bed (probeer hiervan een inschatting te maken)?

Gemiddeld ongeveer keer per week.

13. De stellingen in de tabel hieronder gaan over uw mening over het wassenzonder-water concept. Het maakt daarbij niet uit of $u$ zelf ervaring heeft met het gebruik van wassen-zonder-water of niet.

Per stelling kunt $u$ aangeven in hoeverre $u$ het ermee eens bent door naast elke stelling een kruisje te zetten in de kolom van uw antwoord. Er zijn geen goede of foute antwoorden, het gaat om uw mening over wassen-zonderwater. Wel is het belangrijk om ook bij deze stellingen er rekening mee te 
houden dat het gaat over het gebruik van wassen-zonder-water voor de volledige wasbeurt op bed van cliënten.

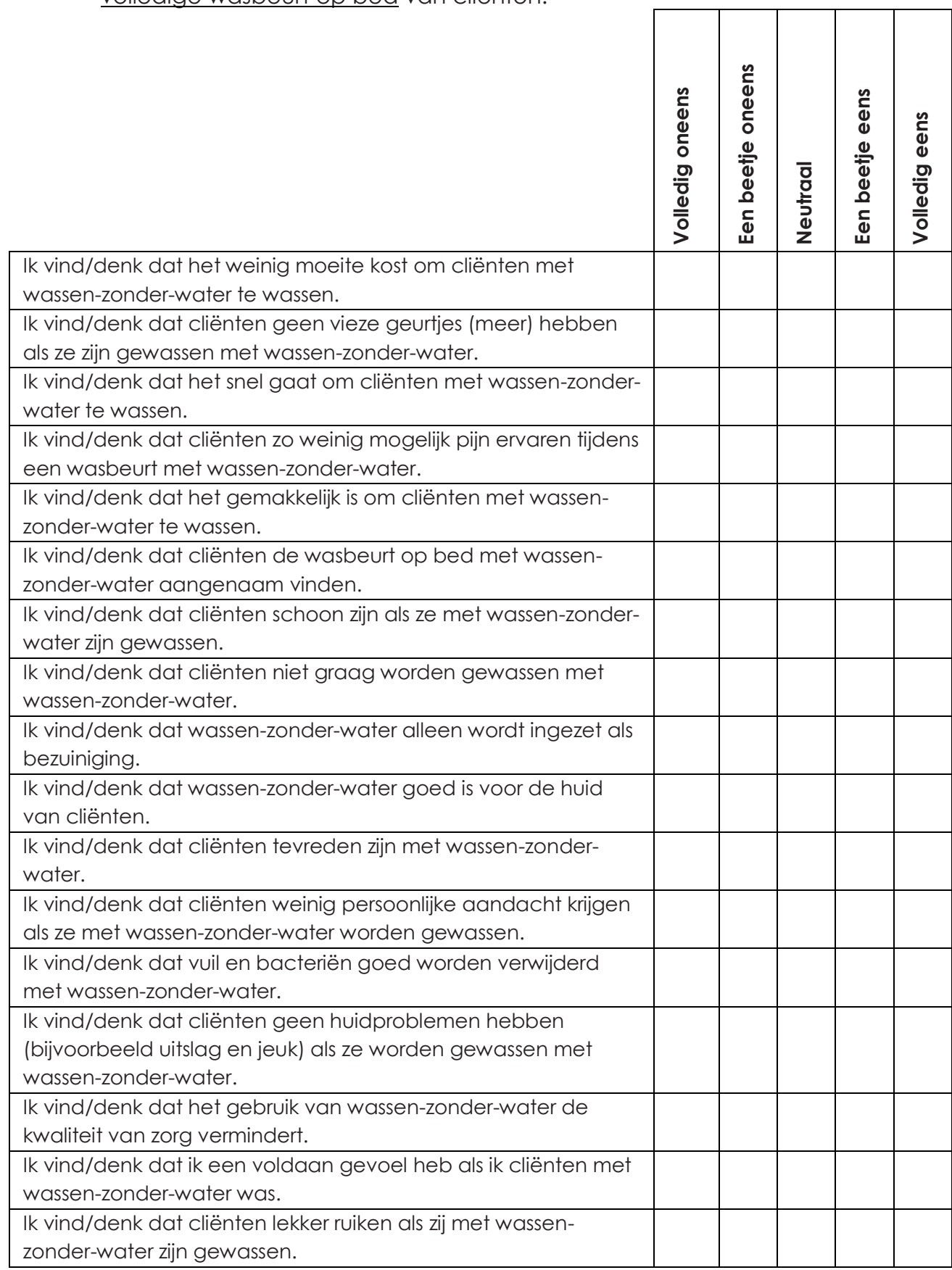

Dit is het einde van de vragenlijst. Heel erg bedankt voor uw medewerking. 


\section{ACKNOWLED GMENTS}


So... most work has been done, now it is time for the toughest part: the acknowledgements. This is not tough because I have no one to thank, quite the contrary, I have to remember all the persons who contributed directly or indirectly to this dissertation. Therefore, I want to start with a disclaimer right away: if you are not mentioned in these acknowledgements that is (probably) not because you are not important to me, but simply because I forgot to mention you. Unlike many others, it took me 6 years to finish this dissertation so I might have forgotten some people. I would like to offer these people an opportunity to be included here so:

Dear (fill out your name), thank you for your support, without you, this dissertation (and therefore, this chapter) would not have existed!

Of the people I do remember $:$, I like to start to thank every member of the supervisory team, ladies first.

Sandra, thank you for your continuous support in the conduct of my research. You were my navigator in the world of science and your help, tips and tricks uplifted the quality of my/our research. Although you must have been under constant time pressure (given that we mostly started our meetings a bit too late), you always gave me the feeling that you had plenty of time for me and I never felt rushed. I also appreciate your hands-on attitude, which came in very useful for example during several rounds of data collection for several studies. I am certain that many people, health care practice and science will benefit from your appointment as a professor in nursing science specialized in geriatric care at home!

"Service professor" Gaby, you were my first manager after I obtained my Master degree and I am glad that I contacted you again to be part of my supervisory team. I am very grateful for your patience, honest feedback (whether positive or constructive), positivism and perseverance. I also want to thank you for your commitment to this project, even though you went for a sabbatical at the other side of the globe, I still had the opportunity (actually even more than before) to meet with you regularly (and see a glimpse of Australian "golden M" restaurants). You have to know that made me feel special and I highly enjoyed our meetings, whether one-onone (from both sides of the world, at your home or at the university) or in our team meetings.

"Nursing professor" Jan, next to your valuable and clear comments about the research, you are one of the friendliest persons I know. While writing this, I even wonder if anger is an emotion that you are familiar with. In the team that consisted of only calm people, you always appeared to be the most laid-back. Still, after listening to my thoughts and ideas, you always asked the right questions that made me reflect on my thoughts. Sometimes, your comments were the opposite of comments that you made before. My immediate reaction to that was sometimes one of slight frustration but in the long-term it helped me realize that the world is never black or white (even 
not for a colorblind person like me (:). I am looking forward to meet you in the front row of a Muse concert (hopefully that is possible again soon).

To the whole team I would like to say that I have always appreciated our meetings although sometimes, your compliments made me feel a bit uncomfortable. Furthermore, although it was tough sometimes, I do not regret that we started (and finished!) our research with a multidisciplinary team. The discussions during our team meetings in which the nursing and service science fields mingled have been very inspirational and I hope you enjoyed it as much as I did. The last thing I want to say about the team is that you all showed that science can be very practically oriented, which was exactly what I was hoping to find in the team.

Although not officially part of the scientific team, I also want to thank of course Erik who gave me the chance to start a PhD. Even though it did not go completely as planned (it took two years longer and the research turned out to be less international as intended), and I am sure that you must have been inpatient at some stages, you always supported me and offered me the freedom to finish this piece of work. I cannot thank you enough for your patience, support and personal interest in me and my research. It felt good to have you on my side during the meetings with the team, which compensates for the meetings in which you stole my time by mentioning things that were off-topic ;) I am looking forward to the years ahead in Team Tomorrow because I know that we can work together on a lot of interesting projects.

I would also like to thank the members of my assessment committee Prof. Jos Schols, Prof. Jos Lemmink, Prof. Trudy van der Weijden, Prof. Lisette Schoonhoven and Dr. Benjamin Olivares Bøgeskov for evaluating my dissertation.

Next, I want to thank all my colleagues at Arion who showed an interest in my research and who tried to leave me alone on my "University-days". In particular, I want to thank two of the most empathic and therefore most appreciated colleagues (or rather friends): Davy and Marco. Davy, Marco, thanks for being the "empathree" together with me and for all the laughs but also for all your help and support. Davy, thanks for helping me make sense of statistics and Marco, thanks for sharing the "nurseperspective" when we talked about my research. You both helped me a lot, also in terms of relaxing during table-tennis matches and during our weekly Friday afternoon drinks, which have always been the perfect start of my quarantine-weekends. I also want to thank Saskia for helping me out on the look and feel of this dissertation. Saskia, I am sure that the message comes across better because of your visuals.

Then a special thanks to the university community! Although I am an external PhD student (candidate sounds better), and I never was "officially" part of the Service Science Factory, the Marketing and Supply Chain Management department, or the Health Services Research department, you always gave the impression that you did 
see me as any regular part of the crew. In the first place, the department managers and the secretariats of all these departments have always made me feel welcome. I would particularly like to thank Pascal and Joanna for their help. I often did not even have to speak my questions out loud because you already provided the answers. Thanks for making my research a smooth ride! In general, all colleagues at all departments made me feel "belonged" in the limited time that I was present at the university, thank you all!

A special thanks goes out to all my roomies over the years, starting with my roomies at HSR. Mariska, you have been my first roomie and I am happy that we could explore what it means to start a PhD together. We shared important experiences, for example, it was you who bluntly shared the news that Johan Cruijf died. With your lack of knowledge about soccer (PSV blugh!), you could not have known that that news would have an impact on me but you helped me cope with it. Gijs, you joined Mariska and me and proofed to be a valuable addition to the room and a top professional! You were always willing to share your point of view no matter the subject. You own the perfect balance of being a serious and conscious scientist and being a friendly and fun guy to hang out with. Unfortunately, your (and my) personality was not sufficient for Annick to stay (the second girl that we saw leaving our room and academia). Annick, you were "one of the guys" right from the start. I highly enjoyed your (too short) presence and are thankful for your help in my research. Dennis, you were the last person that joined our room. I know it was not always easy for you to stay focused in the most crowded room of the building but I think you showed your remarkable skills of being extremely goal-focused and an extra-ordinary multi-tasker. I hope you will find a good balance in everything that is on your plate. Thank you for your willingness to help, even though you had enough to deal with on your own already.

At the Marketing and Supply Chain Management department, I had less roomies but sometimes quality is more important than quantity. Alex thank you for your down-toearth and no-nonsense look at academia that was very welcome sometimes to put things in perspective. I might have been the 'fifth wheel' as I joined you and Robert but I never felt that when we sat together in one room and regularly had a good laugh. Robert, you became more than a roommate, most of everyone, you became my mentor. Not only in science, but also in the world of movies and music. You enriched my academic journey, but perhaps even more contributed to my (hopefully) improved taste for movies and music. I do not only speak for myself but also for Wendy and Quinn (and Dex I guess) when I say that we enjoyed having you around.

Next to my roommates, there are a few other persons I want to thank in particular. Of course, Martina, you are on top of this list. Whereas your soulmate was my mentor, you were my ultimate scientific companion. I cannot imagine there is anyone on this 
earth who would have been a better person to live through academic struggles. Thank you a lot for being the icing of the cake for many Wednesdays at which I had the best lunch meetings of my life (or at least the working life). I want to thank Kars for the fun we had, especially during the academic course of which I cannot even remember the name or content again. Finally, thank you for all of you who helped me out at different stages of my research: Erica, Merel, Svenja, Roy, Katya, Theresa, Audrey, Jules, Hanneke, Mark, Stan, Ruud, and Rixt. Frans, thank you for your patient and clear help in the statistical analyses. Ramon, thank you for the inspiring discussion on qualitative research.

Also outside academia, many people made my research possible of which I want to thank one person in particular: Marijke Hommerich. Marijke, thank you for being an ambassador of my research at Zuyd and for arranging the data collection at different points in time during three academic years. If it was not for you, I would have stopped the data collection already after the first time but you convinced me every time that we had to run new experiments and proofed that you were right.

Enough about people at work, time for family and friends. I am lucky to have plenty of people around me that make me realize that there are much more important things in life than work. Without all these people, I would not have the energy and motivation to work at all. So, thank you to my friends from the band (Vincent, Gerard, Paul, Martijn and Jos), my sport buddies but above all the summer-bba/social events/Christmas eve/borrel-friends group: Mark and Aimee (the glue and nicelychaotic home base of the group), Laurens and Willemien (I am still not over the fact that you moved to Amsterdam/Abcoude), Lotje (my niece but even more my lifetime friend and the reason why I am with Wendy), Eva and Bas, Mariëlle, Mark and Kirsten (Mark, that is why I did not mention you when I mentioned the band), Rik and Mariëlle, Tuur, and Erwin. Also thanks to Jesper (who used to be part of the crew) for always showing great interest in my research. I am blessed with having you as friends, and look forward to be the "Ross".

Of course, Sanne, my sweet sister, you are also part of the abovementioned friendsgroup but you are more than a friend, we also have a blood-tie that becomes visible in our dark/strange sense of humor. Thank you for that! Ruben, thank you (and thank you Marieke) for starting a restaurant/bar in Valkenburg (to everyone who reads this: go to "Bij de Jongens" in Valkenburg), which became my favorite spot to relax and enjoy a nice beer! Both of you (and actually many others mentioned under family and friends) did not contribute to my research at all but apparently, an acknowledgement chapter is not complete without thanking siblings so thank you!

Thank you to all my parents in law, Joke, Hub, Dré and Sandra for all the nice trips, dinners, lunches, home improvement projects, and visits. Thank you Bryan for sharing my enthusiasm for John Mayer and System of a Down (the perfect playlist). 
A special word of thanks goes of course to my parents. Mom, thank you for being a great mother, grandmother and colleague. Thank you for your unstoppable interest and involvement in my work. You always (asked or unasked) offered support in finding my way in academia. Sorry that I sometimes reacted annoyed if you asked about my research and I did not want to talk about it. I felt bad about my reaction many times because is it not fair to you. I am glad that this did not withhold you from staying involved, thank you! Also thanks to you dad for not asking about my research and therefore being a great opposite to mom (-). We don't talk a lot about our feelings for each other, therefore I want to take this opportunity to thank you for being a great dad, which I am reminded of every time I see you with Quinn. You are still the person who I can ask for practical help (to get a lift, fix a door, whatever) and who will be there in a minute. I am glad that I inherited your Dutch common sense. I love the both of you very much.

Of course, the final part is devoted to my own little family. Wendy, thank you for sticking with me for already 10, 11, 12, 13, 14, 15, 16, 17, 18, 19, 20 (the right number must be one of these) years. During this time, you have been (with varying success) my flirt, girlfriend, wife, friend, Salsa dance partner, tennis partner, festival and concert companion, fellow Ajax supporter (although for you it was mainly about Lasse Schöne), game contestant, travel companion, drinking buddy, and of course parent of our lovely daughter Quinn. You are my better half in many things. Thank you very much for sticking around with me despite my weaknesses, such as my pessimistic episodes (which actually is called realism). In relation to this thesis, I want to thank you for giving me the opportunity to spend all the time that I needed on my research. Although you have a very successful career yourself, you are way better than me in combining that with family and social life. I will try to learn from you but for now thanks for making that possible. I do not think I do a very bad job in parenting, but you are a better parent for sure and that makes me love you even more!

The final words are for my daughter Quinn. Wat een cliché (iets dat veel mensen zeggen maar wel waar is) maar jij geeft mij elke dag zo veel (geluk en blijdschap vooral). Werk is harstikke leuk maar soms gaat niet alles zoals ik het zou willen en kan ik mij daar best een beetje boos of verdrietig over voelen. Maar dan kom ik thuis en knuffel en klets jij al mijn zorgen weg. Jouw vrolijkheid werkt aanstekelijk (word ik ook vrolijk van) en ik ben super-mega-trots op je en zal dat altijd blijven of je nu ijsbolletjesschepper, juffrouw, of dokter wordt. Ik hoop vooral dat je zo vrolijk en de knuffelkont blijft die je nu bent. Van jou houd ik denk ik het allermeest; zo veel dat als ik een liedje hoor over dochters, zoals 'Daughters' van John Mayer, zelfs ík daar sentimenteel (een aansteller) van word. Ik hoop dat je net zo trots bent op mij. 
ABOUT THE

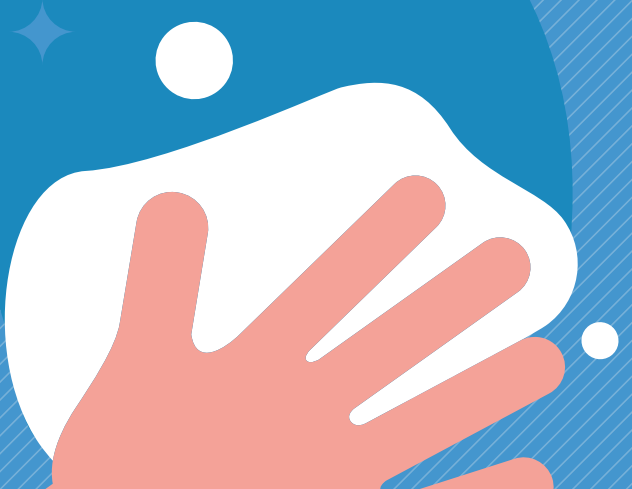


Fabian Groven was born in Heerlen, The Netherlands, on the 15th of November, 1983. Fabian obtained his Bachelor of Science degree in International Business in 2005, after which he started his Masters in International Business with the Marketing specialization, which he finished in 2006. He obtained both the Bachelor and the Master degree at Maastricht University.

After shortly working as a tutor at Maastricht University until April 2007, Fabian worked for several commercial companies, including Randstad (as a job mediator), 3W real estate (as a market researcher), and his current employer Arion. At Arion, Fabian has been a New Business Employee, a Product \& Research Manager, an Advisor to the CEO, and a Corporate Social Responsibility (CSR) Manager. While he was an employee of Randstad, he contributed to a publication of his Master thesis about the influence of background music on Front-Line Employees in the Marketing Yearbook of 2008. In March 2015, Fabian started as a part-time external PhD candidate at the department of Health Services Research of the Care and Public Health Research Institute (CAPHRI) and the department of Marketing \& Supply Chain Management of the School of Business and Economics of Maastricht University.

Between March 2015 and June 2021, Fabian worked on a research project about the bed bath and the use of the washing without water intervention for this health care activity in specific. For this research project, Fabian explored the general importance of the bed bath and studied the effect of the use of the washing without water intervention on health care actors' experiences with the bed bath. During the conduct of his PhD, he presented his research and results at several scientific conferences, industry events, and health care institutions. 


\section{SCIENTIFIC PUBLICATIONS}




\section{Publications}

- Groven FMV, Hamers JPH, Odekerken-Schröder G, and Zwakhalen SMG. Nurses' beliefs about the bed bath: Testing the self-developed Bed Bath Beliefs tool - A methodological study. Submitted.

- Groven F, Odekerken-Schröder G, Zwakhalen S, and Hamers J. Network wellbeing from a balanced centricity perspective. Journal of Services Marketing 2021;35:1-14.

- Groven FM, Zwakhalen SM, Odekerken-Schröder G, Tan, F, and Hamers JP. Comfort during the bed bath - A randomised crossover trial on the effect of washing without water versus water and soap in nursing students. Journal of Clinical Nursing 2021;30:2234-2245.

- Groven FM, Zwakhalen SM, Odekerken-Schröder G, Tan, F, and Hamers JP. The effects of washing without water versus the traditional bed bath with water and soap on comfort and physical demands: protocol of a cross-over randomized trial. 2019. Available online: www.researchsquare.com/article/rs7409/v1.

- Groven FMV, Zwakhalen SM, Odekerken-Schröder G, Joosten, EJ, and Hamers JP. How does washing without water perform compared to the traditional bed bath for bedridden patients: a systematic review. BMC Geriatrics 2017;17:1-16.

- Zwakhalen S, Metzelthin S, Groven F, Machiels M, and Hamers J. Samenwerking in Academische netwerken - Wassen zonder water. Innoveren in de dagelijkse pratkijk. Tijdschrift voor Ouderengeneeskunde 2017;6.

- Van Birgelen MJH, Groven F, Semeijn J, Bloemer, JMM. Wat is de invloed van het volume van achtergrondmuziek op de emoties, het denken en het gedrag van supermarktmedewerkers? Ontwikkelingen in het Marktonderzoek: Jaarboek 2008:227-247. 


\section{IVING LAB IN AGEING AND LONG-TERM CARE}




\section{LIVING LAB IN AGEING AND LONG-TERM CARE}

This thesis is part of the Living Lab in Ageing and Long-Term Care, a formal and structural multidisciplinary network consisting of Maastricht University, nine long-term care organizations (MeanderGroep Zuid-Limburg, Sevagram, Envida, Cicero Zorggroep, Zuyderland, Vivantes, De Zorggroep, Land van Horne \& Proteion), Intermediate Vocational Training Institutes Gilde and VISTA college and Zuyd University of Applied Sciences, all located in the southern part of the Netherlands. In the Living Lab we aim to improve quality of care and life for older people and quality of work for staff employed in long-term care via a structural multidisciplinary collaboration between research, policy, education and practice. Practitioners (such as nurses, physicians, psychologists, physio- and occupational therapists), work together with managers, researchers, students, teachers and older people themselves to develop and test innovations in long-term care.

\section{ACADEMISCHE WERLPLAATS OUDERENZORG ZUID-LIMBURG}

Dit proefschrift is onderdeel van de Academische Werkplaats Ouderenzorg Limburg, een structureel, multidisciplinair samenwerkingsverband tussen de Universiteit Maastricht, negen zorgorganisaties (MeanderGroep Zuid-Limburg, Sevagram, Envida, Cicero Zorggroep, Zuyderland, Vivantes, De Zorggroep, Land van Horne \& Proteion), Gilde Zorgcollege, VISTA college en Zuyd Hogeschool. In de werkplaats draait het om het verbeteren van de kwaliteit van leven en zorg voor ouderen en de kwaliteit van werk voor iedereen die in de ouderenzorg werkt. Zorgverleners (zoals verpleegkundigen, verzorgenden, artsen, psychologen, fysio- en ergotherapeuten), beleidsmakers, onderzoekers, studenten en ouderen zelf wisselen kennis en ervaring vit. Daarnaast evalueren we vernieuwingen in de dagelijkse zorg. Praktijk, beleid, onderzoek en onderwijs gaan hierbij hand in hand. 


\section{PHD-THESES LIVING LAB IN AGEING AND LONG-TERM CARE/PROEFSCHRIFTEN ACADEMISCHE WERKPLAATS OUDERENZORG ZUID-LIMBURG}

Fabian Groven. The bed bath with or without water? It's a wash! Experiences with the washing without water intervention used for the bed bath. 2021

Sascha Bolt. The fundamentals of a DEDICATED palliative approach to care for people with dementia. 2021

Angela Mengelers. To risk or to restrain? Involuntary treatment use in people with dementia living at home. 2021

Katya Sion. Connecting Conversations. Experienced quality of care from the resident's perspective: a narrative method for nursing homes. 2021

Linda Hoek. Change begins with choice. Supporting the autonomy of nursing home residents with dementia through partnership. 2020

Mirre den Ouden. Every step counts. Daily activities of nursing home residents and the role of nursing staff. 2018

Theresa Thoma-Lürken. Innovating long-term care for older people. Development and evaluation of a decision support app for formal caregivers in community-based dementia care. 2018

Eveline van Velthuijsen. Delirium in older hospitalised patients: diagnosis and management in daily practice. 2018

Bram de Boer. Living at a green care farm. An innovative alternative for regular care in nursing homes for people with dementia. 2017

Nienke Kuk. Moving forward in nursing home practice. Supporting nursing staff in implementing innovations. 2017

Irma Everink. Geriatric rehabilitation. Development, implementation and evaluation of an integrated care pathway for older patients with complex health problems. 2017

Ramona Backhaus. Thinking beyond numbers. Nursing staff and quality of care in nursing homes. 2017

Martin Van Leen. Prevention of pressure ulcers in nursing homes, a big challenge. 2017 
Mariëlle Daamen-Van der Velden. Heart failure in nursing home residents. Prevalence, diagnosis and treatment. 2016

Armand Rondas. Prevalence and assessment of (infected) chronic wounds. 2016

Hanneke Beerens. Adding life to years. Quality of life of people with dementia receiving long-term care. 2016 (Cum Laude)

Donja Mijnarends. Sarcopenia: a rising geriatric giant. Health and economic outcomes of community-dwelling older adults with sarcopenia. 2016

Tanja Dorresteijn. A home-based program to manage concerns about falls. Feasibility, effects and costs of a cognitive behavioral approach in community-dwelling, frail older people. 2016

Basema Afram. From home towards the nursing home in dementia. Informal caregivers' perspectives on why admission happens and what they need. 2015

Noemi Van Nie-Visser. Malnutrition in nursing home residents in the Netherlands, Germany and Austria. Exploring and comparing influencing factors. 2014

Esther Meesterberends. Pressure ulcer care in the Netherlands versus Germany 0-1. What makes the difference? 2013

Math Gulpers. EXBELT: expelling belt restraints from psychogeriatric nursing homes. 2013

Hilde Verbeek. Redesigning dementia care. An evaluation of small-scale homelike care environments. 2011

Judith Meijers. Awareness of malnutrition in health care, the Dutch perspective. 2009

Ans Bouman. A home visiting program for older people with poor health. 2009

Monique Du Moulin. Urinary incontinence in primary care, diagnosis and interventions. 2008

Anna Huizing. Towards restraint free care for psychogeriatric nursing home residents. 2008

Pascalle Van Bilsen. Care for the elderly, an exploration of perceived needs, demands and service use. 2008 
Rixt Zijlstra. Managing concerns about falls. Fear of falling and avoidance of activity in older people. 2007

Sandra Zwakhalen. Pain assessment in nursing home residents with dementia. 2007 




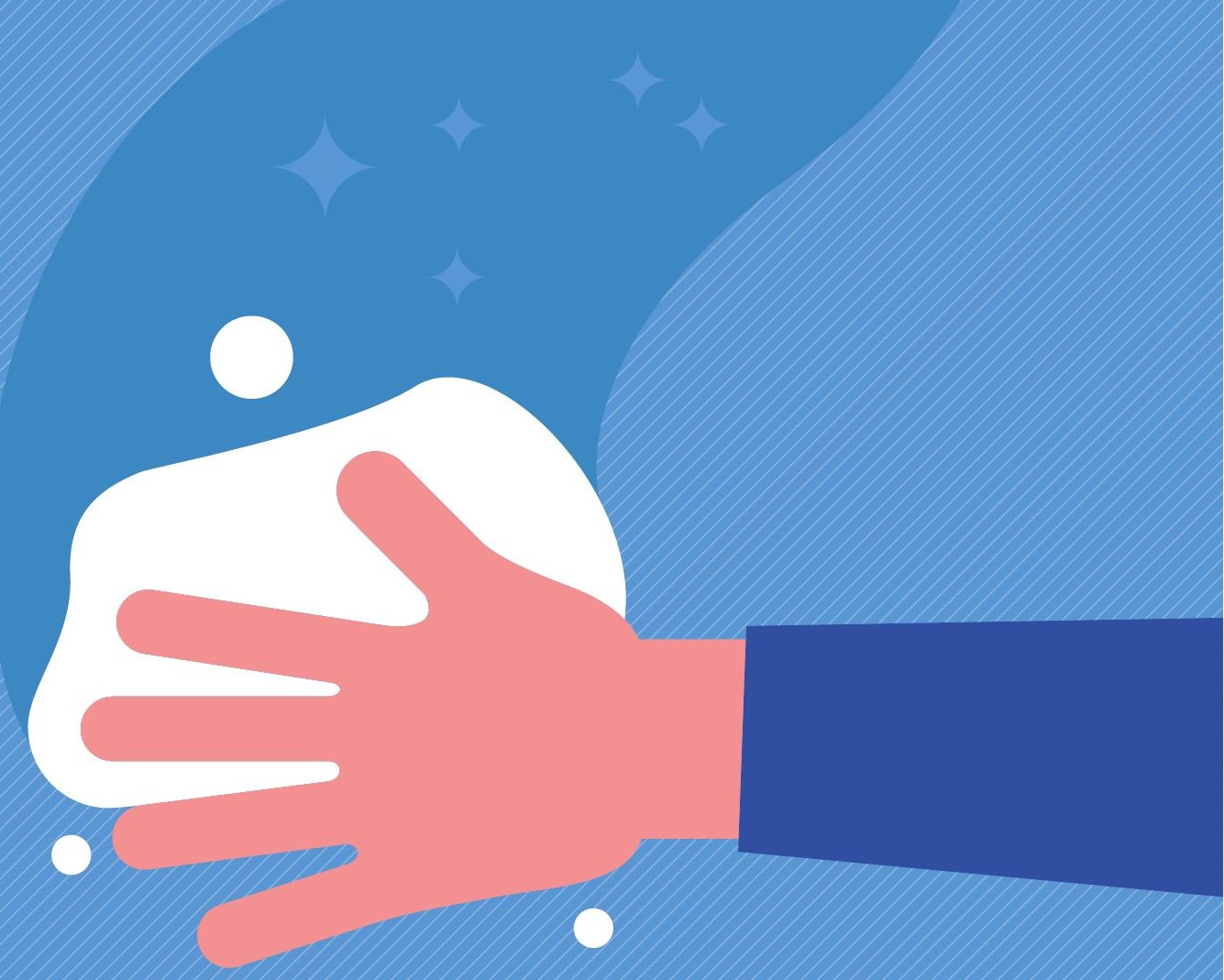

It's a wash laliom

use d to say that something is. equal and that one side does. not have an advantage. 